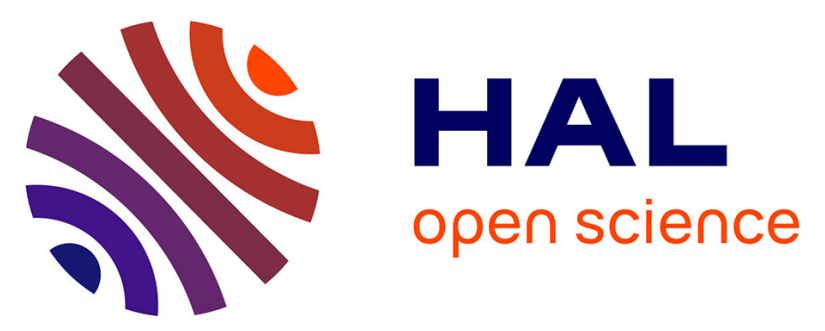

\title{
Troubles post-traumatiques chez des sujets ayant été victimes de violences sexuelles avant l'âge de 15 ans : aspects cliniques, thérapeutiques et médico-économiques
} Sophie Vacher-Boulogne

\section{- To cite this version:}

Sophie Vacher-Boulogne. Troubles post-traumatiques chez des sujets ayant été victimes de violences sexuelles avant l'âge de 15 ans: aspects cliniques, thérapeutiques et médico-économiques. Médecine humaine et pathologie. 2013. dumas-01131521

\section{HAL Id: dumas-01131521 https://dumas.ccsd.cnrs.fr/dumas-01131521}

Submitted on 13 Mar 2015

HAL is a multi-disciplinary open access archive for the deposit and dissemination of scientific research documents, whether they are published or not. The documents may come from teaching and research institutions in France or abroad, or from public or private research centers.
L'archive ouverte pluridisciplinaire HAL, est destinée au dépôt et à la diffusion de documents scientifiques de niveau recherche, publiés ou non, émanant des établissements d'enseignement et de recherche français ou étrangers, des laboratoires publics ou privés.

\section{(이) $\$$}

Distributed under a Creative Commons Attribution - NonCommercial - NoDerivatives| 4.0 


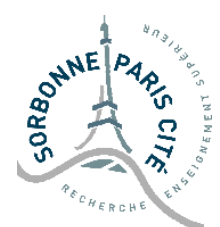

\section{AVERTISSEMENT}

Cette thèse d'exercice est le fruit d'un travail approuvé par le jury de soutenance et réalisé dans le but d'obtenir le diplôme d'Etat de docteur en médecine. Ce document est mis à disposition de l'ensemble de la communauté universitaire élargie.

Il est soumis à la propriété intellectuelle de l'auteur. Ceci implique une obligation de citation et de référencement lors de l'utilisation de ce document.

D’autre part, toute contrefaçon, plagiat, reproduction illicite encourt toute poursuite pénale.

Code de la Propriété Intellectuelle. Articles L 122.4

Code de la Propriété Intellectuelle. Articles L 335.2-L 335.10 


\section{UNIVERSITÉ PARIS DESCARTES \\ Faculté de Médecine PARIS DESCARTES}

Année 2013

\section{THÈSE \\ POUR LE DIPLÔME D'ÉTAT \\ DE \\ DOCTEUR EN MÉDECINE}

Troubles post-traumatiques chez des sujets ayant été

victimes de violences sexuelles avant l'âge de 15 ans : aspects cliniques, thérapeutiques et médico-économiques

\section{Présentée et soutenue publiquement}

le $1^{\mathrm{er}}$ octobre 2013

$$
\text { Par }
$$

\section{VACHER-BOULOGNE, Sophie}

Née le 25 juin 1983 à Pointe-à-Pitre (971)

\section{Dirigée par M. Le Professeur Jehel, Louis}

Jury :

M. Le Professeur Granger, Bernard Président

M. Le Professeur Baubet, Thierry Membre Mme Le Docteur Abgrall-Barbry, Gaëlle Membre 


\section{Remerciements}

A mon directeur, le Professeur Jehel, de sa confiance en me soumettant ce sujet, au Professeur Granger de présider le jury, au Professeur Baubet et au Docteur Abgrall-Barbry qui se sont rendus disponibles pour la soutenance.

Je vous remercie de l'honneur que vous me faites en acceptant de juger mon travail.

Pour ma formation un grand merci à tous mes maîtres de stage :

Au Pr Consoli S., aux Dr Guedj M-J., Guillibert E. et Lemogne C., de m'avoir ouvert les portes de la psychiatrie au cours de mon externat. Au Dr Dierzynski N. et au Dr Gouttefangeas F., de m'avoir mis le pied à l'étrier de cette spécialité. Au Pr Jehel L. et au Dr Abgrall-BarBry G., d'avoir suscité mon intérêt pour le psychotraumatisme et au Dr Piernikarch, pour la psychiatrie de l'adolescent. Au Dr Pasquier de Franclieu S., dont les conseils avisés, sont toujours prodigués avec une juste pincée de rigueur et une bonne louchée d'humour. Au Dr Baillet P., pour ses éclairages, sa disponibilité et sa bienveillance inégalables envers ses internes. Tous, soyez assurés de ma reconnaissance et de mon profond respect.

Merci aux médecins, psychologues, infirmiers, psychomotriciens, aides soignants, assistants sociaux et secrétaires rencontrés sur mes terrains de stage.

Merci aux Dr Levy et Atsou de leurs conseils au démarrage de ce travail, mais aussi pour l'introduction au travail statistique.

Aux patients ayant accepté de participer à l'étude, merci de vous être rendus disponibles.

Aux Daltons and Co : Aurore, Baptiste, Julien, Kristell et Solenn. Notre amitié initiée sur les bancs des Cordeliers, votre fantaisie comme soutien pour traverser ces années d'études et palier le manque de ma famille éloignée. A mes deux infaillibles fraternelles amies Ingrid et Sophie. Je vous dois les amis un merci tout particulier, pour m'avoir soutenue dans ma spécialisation et même à certains moments, d'y avoir cru plus que moi, tant vous me connaissez.

Je salue au passage, les habitants de la Galaxie Star Wars ayant décidé un temps, de poser leurs pod-racers afin de travailler au château en pédopsychiatrie à Orsay.

A mes parents, ma sœur et toute ma famille. A Karukera, mon île aux belles eaux... 


\section{TABLE DES MATIERES}

PREMIERE PARTIE : VIOLENCES SEXUELLES ET TRAUMATISME PSYCHIQUE, UN TOUR D'HORIZON

1. LES VIOLENCES SEXUELLES DANS L'HISTOIRE, L'ART ET LA LITTERATURE ..................... 10

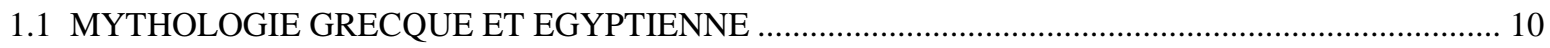

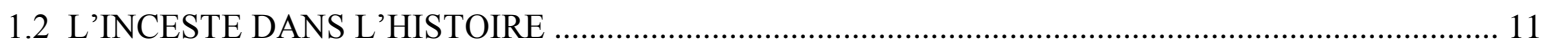

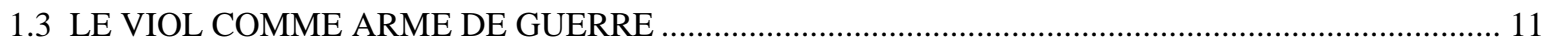

1.4 LES VIOLENCES SEXUELLES DANS LES ROMANS CONTEMPORAINS ……............................... 12

2. HISTORIQUE DE LA NOTION DE TRAUMA EN PSYCHIATRIE : DE L'HYSTERIE A L'ETAT

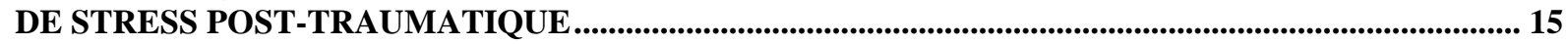

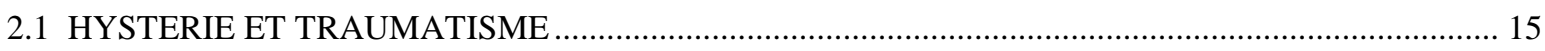

2.2 CONCEPTION JANETIENNE ET PSYCHOPATHOLOGIE DES TRAUMATISMES PSYCHIQUES

2.3 FREUD : DE LA « NEUROTICA », THEORIE DE LA SEDUCTION OU DU TRAUMATISME SEXUEL, A LA THEORIE DU FANTASME OU DE LA SEXUALITE INFANTILE .............................. 19

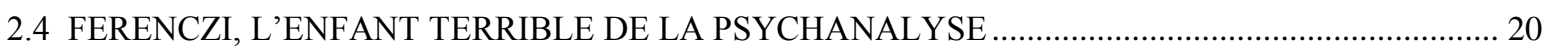

2.5 LES GUERRES DU XX ${ }^{\mathrm{e}}$ SIECLE, LA NEVROSE DE GUERRE ET L'ETAT DE STRESS POST-

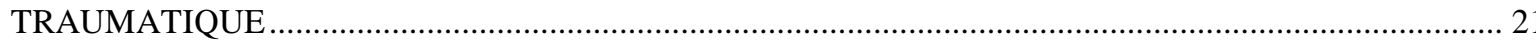

3. COMMENT DEFINIR LES VIOLENCES SEXUELLES ? ................................................................23

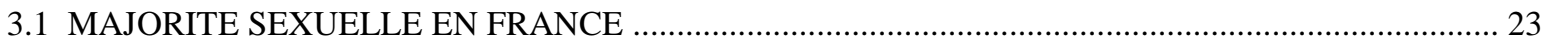

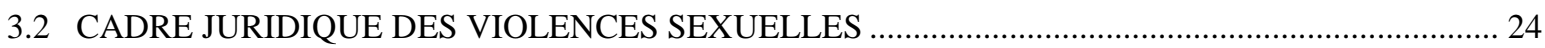

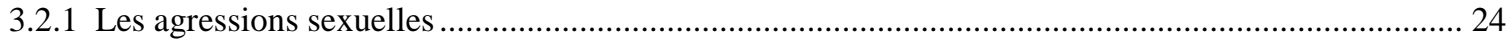

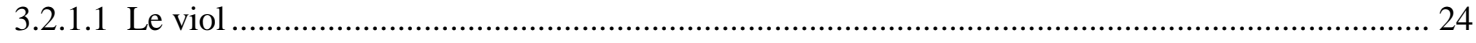

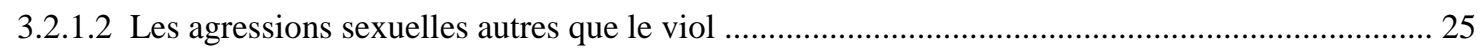

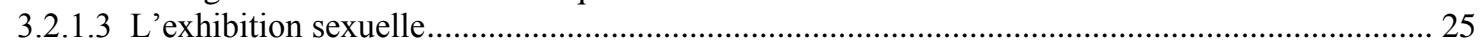

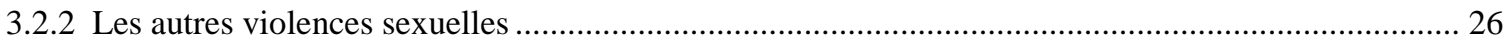

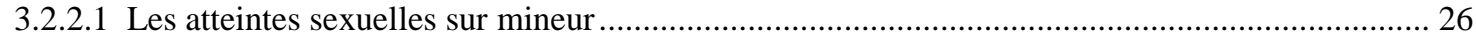

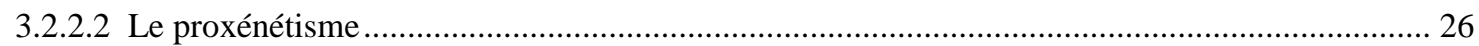

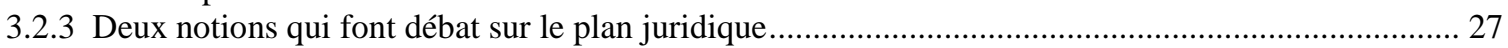

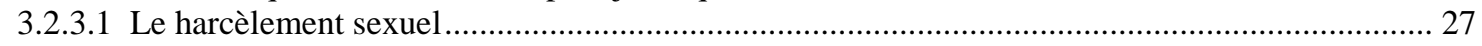

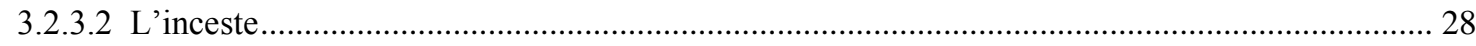

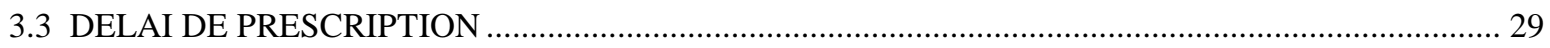

4. EPIDEMIOLOGIE DES VIOLENCES SEXUELLES ................................................................................... 30

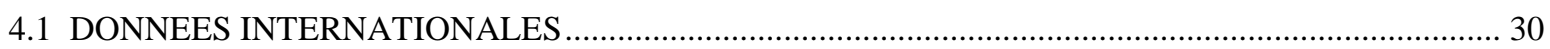

4.2 LES ENQUETES EPIDEMIOLOGIQUES FRANÇAISES ....................................................... 32

4.3 EPIDEMIOLOGIE DES FACTEURS DE RISQUE DE VIOLENCES SEXUELLES SUR MINEUR ... 34

4.3.1 Caractéristiques des agresseurs sexuels sur mineur............................................................. 34

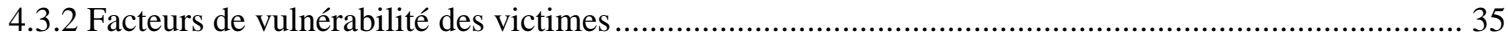


1. LES TROUBLES CHEZ L'ADULTE AYANT ETE VICTIME DE VIOLENCES SEXUELLES DANS

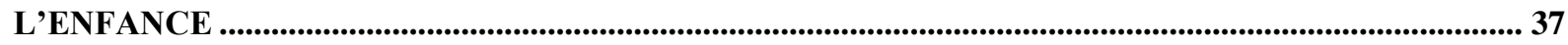

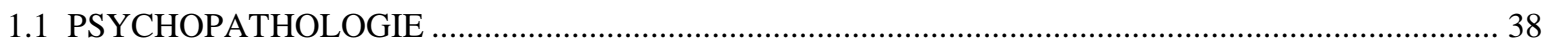

1.1.1 L'Etat de Stress Post-Traumatique (ESPT) et autres troubles névrotiques ................................... 38

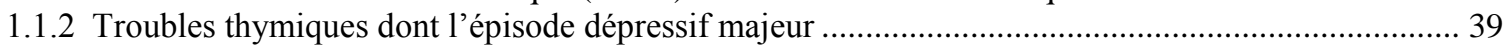

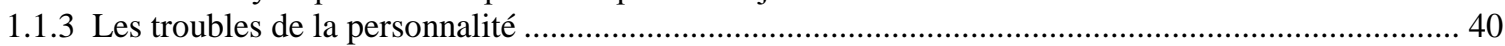

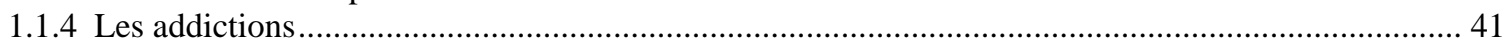

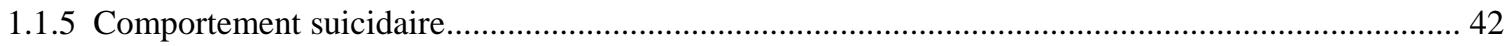

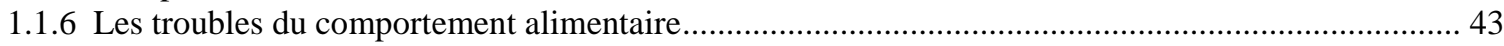

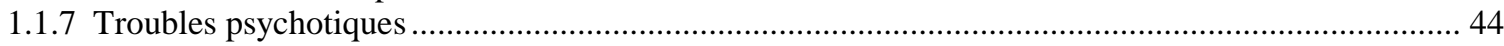

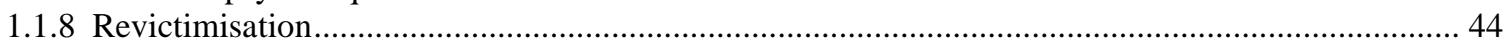

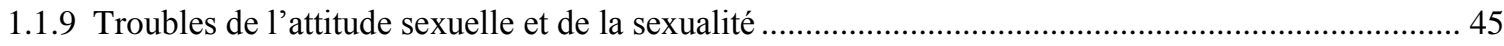

1.1.10 Les séquelles neurobiologiques comme modèle explicatif de la psychopathologie ...................... 46

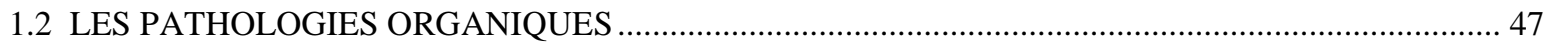

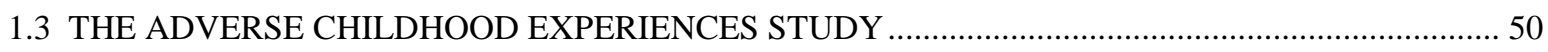

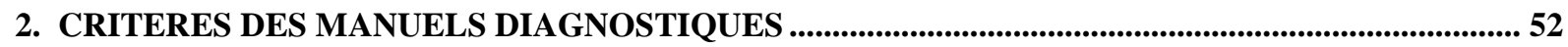

2.1 L'ETAT DE STRESS POST-TRAUMATIQUE (ESPT) DANS LE DSM-IV ....................................52

2.2 L'ETAT DE STRESS POST-TRAUMATIQUE SELON LE DSM-V ........................................... 54

2.3 PLACE DES VIOLENCES SEXUELLES DANS LES MANUELS DIAGNOSTICS ...........................56

3. EVALUATIONS DIAGNOSTIQUE ET PRONOSTIQUE DE L'ETAT DE STRESS POSTTRAUMATIQUE. 57

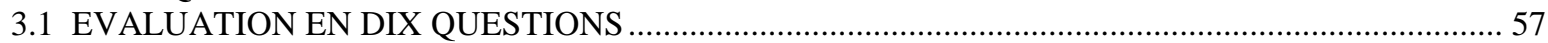

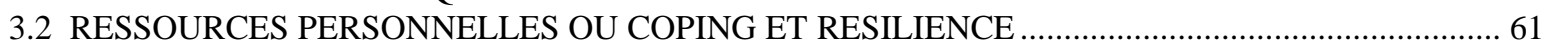

3.3 LES INSTRUMENTS D’ORIENTATION DIAGNOSTIQUE ET D’EVALUATION..........................62

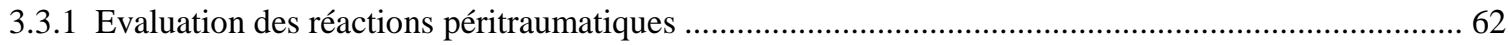

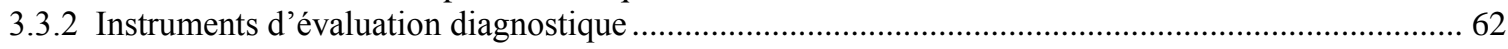

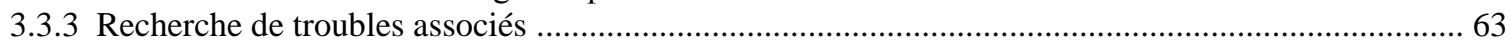

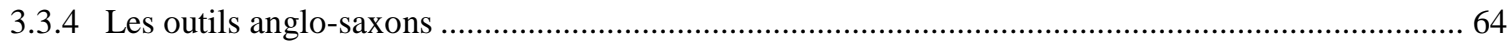

TROISIEME PARTIE : LES TROUBLES POST-TRAUMATIQUES CHEZ L'ADULTE , STRATEGIES

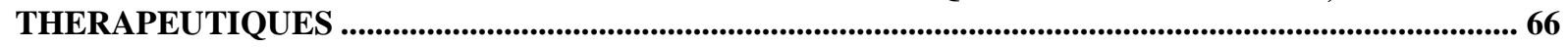

1. MODELES PSYCHOPHATHOLOGIQUES ET PSYCHOTHERAPIES DE L'ETAT DE STRESS

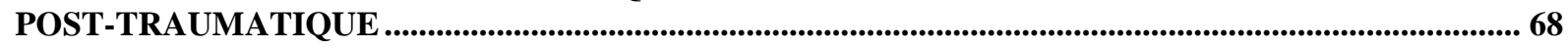

1.1 LES THERAPIES COGNITIVO-COMPORTEMENTALES ET LES THERAPIES DERIVEES .........68

1.1.1 Fondements théoriques des TCC dans la prise en charge de 1'ESPT .........................................69

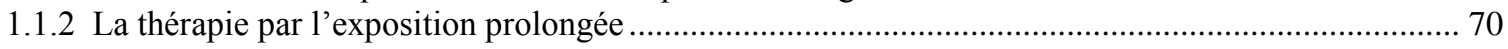

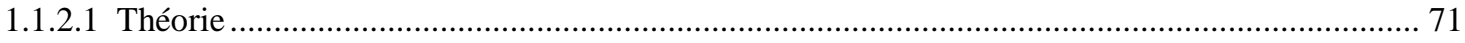

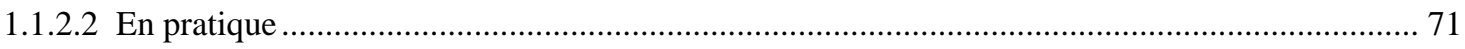

1.1.2.3 Déroulé des séances d'exposition prolongée simple dans le cas d'un viol ............................. 72

1.1.3 Eye Movement Desensitization and Reprocessing (EMDR) ............................................. 74

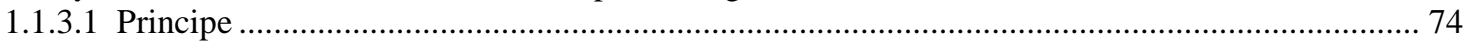

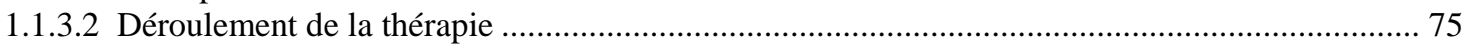

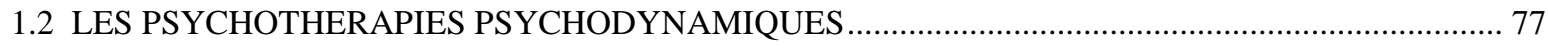

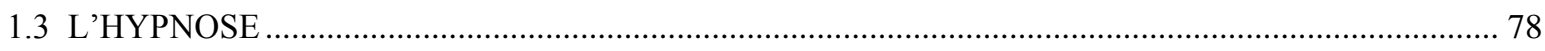

2. COMPREHENSION DE LA NEUROPHYSIOLOGIE DU STRESS ET LA PHARMACOTHERAPIE

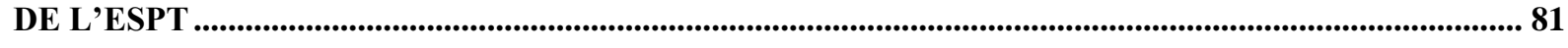




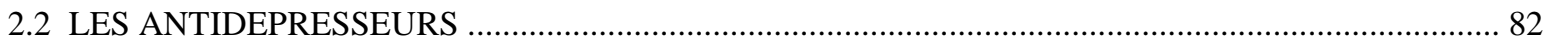

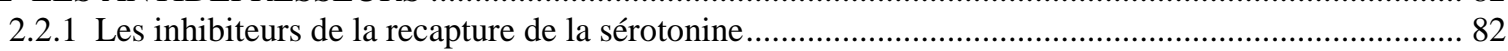

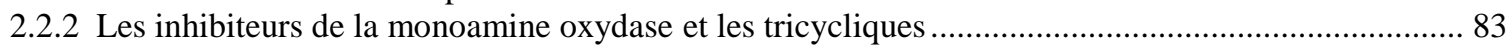

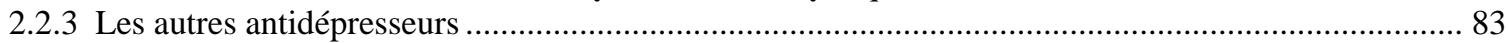

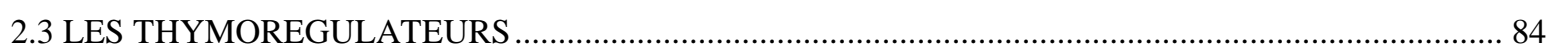

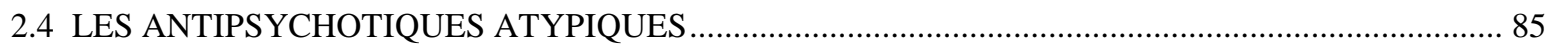

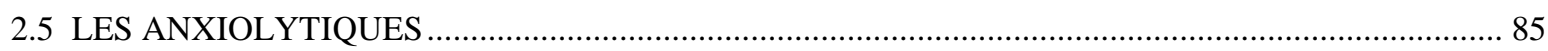

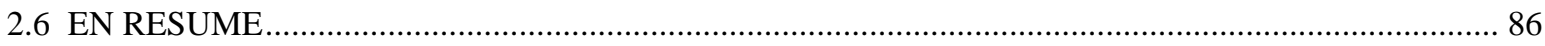

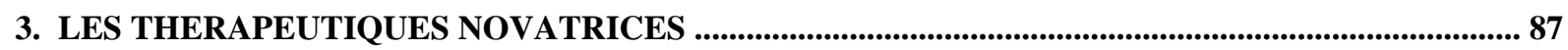

3.1 LES STIMULATIONS MAGNETIQUES TRANSCRANIENNES (r-TMS) ………………….............. 87

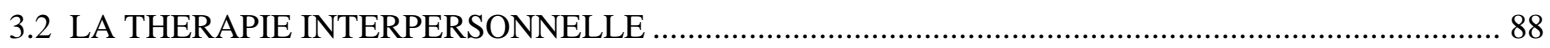

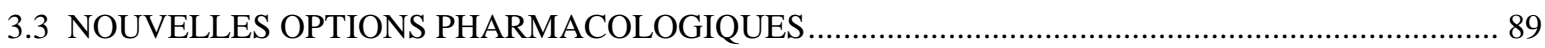

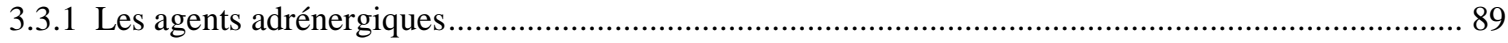

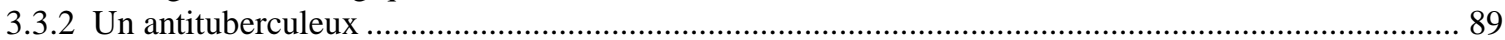

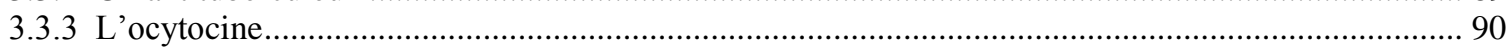

4. LES PUBLICATIONS EVALUANT LES DIFFERENTES THERAPEUTIQUES ................................. 91

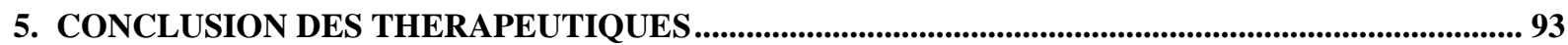

QUATRIEME PARTIE : LES ADULTES AYANT ETE VICTIMES DE VIOLENCES SEXUELLES

AVANT L'AGE DE 15 ANS : APPROCHE MEDICO-ECONOMIQUE ................................................994

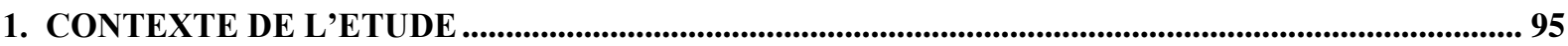

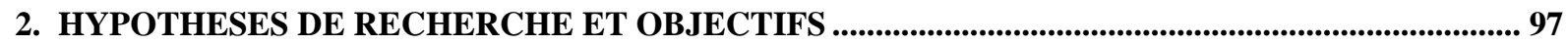

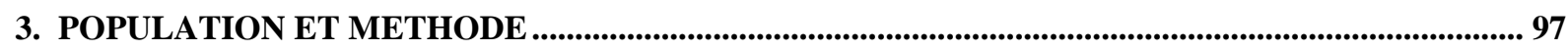

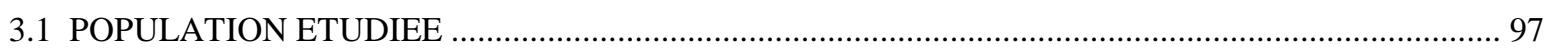

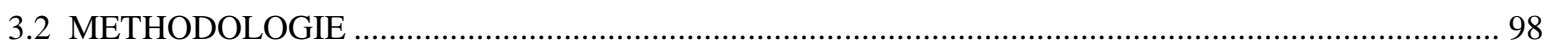

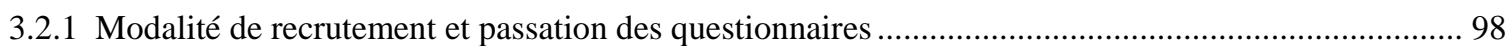

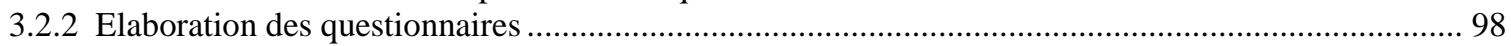

3.2.2.1 Collecte des données socio démographique et de santé globale .......................................... 99

3.2.2.2 Comment évaluer les dépenses de santé ?.......................................................................... 99

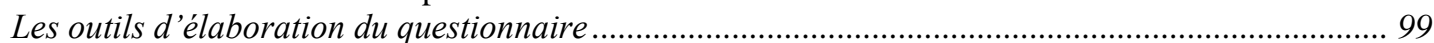

Modalités d'investigation des dépenses................................................................................... 100

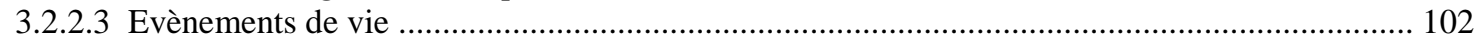

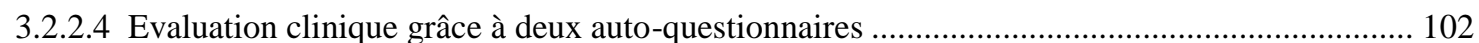

3.2.3 Traitement des informations recueillies grâce aux questionnaires ............................................... 103

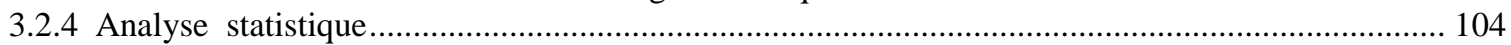

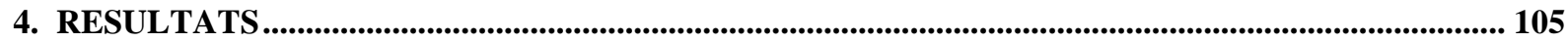

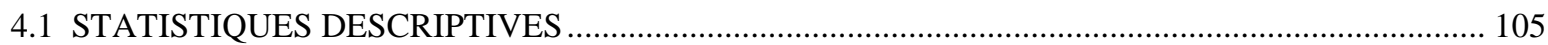

4.1.1 Caractéristiques de la population de l'étude ........................................................................ 105

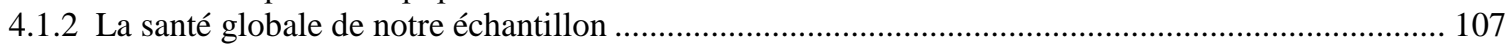




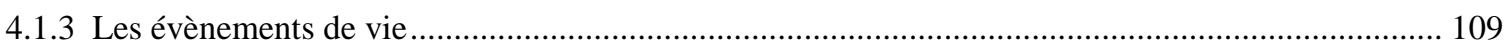

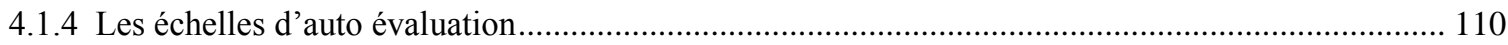

4.1.5 Les dépenses de santé de notre population ............................................................................. 112

4.1.5.1 Calcul des dépenses et données associées obtenues ............................................................. 112

4.1.5.2 Observation de la consommation annuelle de notre population............................................ 115

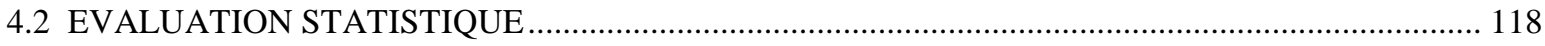

4.2.1 Quelques éléments descriptifs de la population française. .................................................... 118

4.2.2 Comparaison des moyennes annuelles nationales et des moyennes de l'échantillon ...................... 119

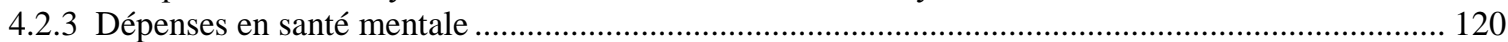

4.2.4 Recherche de facteurs pouvant influencer les dépenses ........................................................... 122

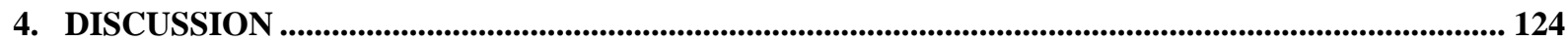

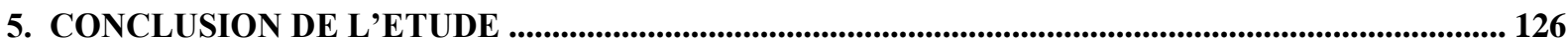

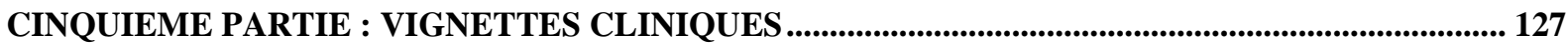

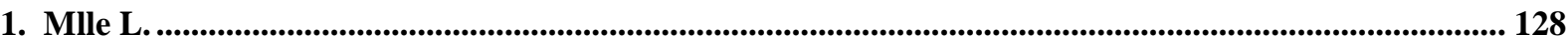

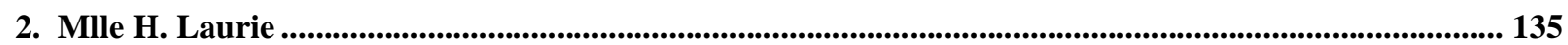

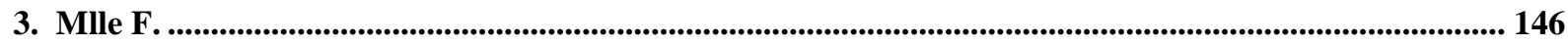

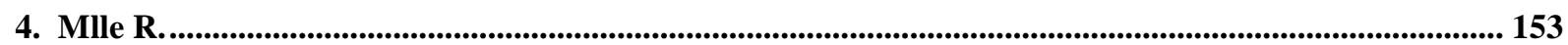

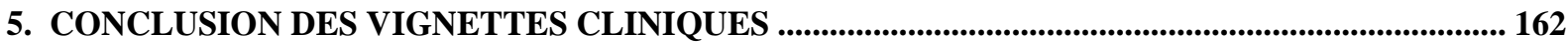

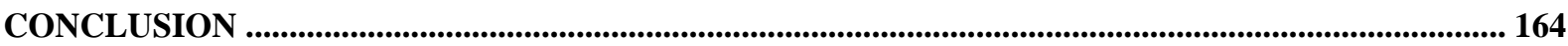

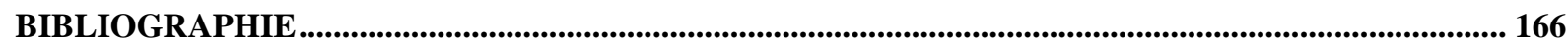

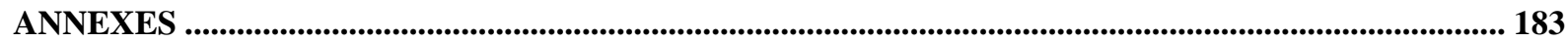

Document annexe 1 : Hétéro-questionnaire : données sociodémographiques, santé globale, recensement des dépenses de santé ......................................................................................................................................................... 184

Document annexe 2 : Beck Depression Inventory (BDI), Echelle de Dépression de Beck .......................... 191

Document annexe 3 : Impact of Event Scale-Revised, IES-R version française............................................. 193 


\section{INTRODUCTION}

Les violences sexuelles, comme nous pouvons le constater dans notre pratique, concernent nombre de nos patients, quelque soit leur tranche d'âge. Une enquête téléphonique réalisée dans la population française retrouve que $16 \%$ des femmes et $5 \%$ des hommes ont vécu au cours de leur vie un rapport sexuel forcé ou une tentative de rapport sexuel forcé. (Bajos 2008) [10]

Ainsi, nous avons été amenés à aider des sujets victimes de tels évènements de vie dans des unités spécialisées en psychotraumatologie mais aussi, de fréquence peut être plus inattendue, dans des centres de consultations et unités d'hospitalisations «classiques » de psychiatrie générale adulte et de pédopsychiatrie.

Si de nos jours, pour les médecins, dans certaines situations, rechercher chez l'enfant une possible maltraitance d'ordre sexuel, ou encore prendre en charge en phase aigue des sujets adultes victimes d'agressions sexuelles, font partie de la pratique, il est beaucoup moins évident d'investiguer dans l'histoire de vie de nos patients adultes, des antécédents de violences sexuelles dans l'enfance. Cette lacune peut être expliquée par le poids du tabou, la crainte de fausses allégations, mais surtout par le peu de place donnée à cette problématique dans les enseignements des formations médicales, paramédicales et sociales. Alors que certains en sont encore à s'interroger sur l'utilité de soulever de tels évènements passés, nous ne pouvons qu'être frappés par leurs répercutions à long terme, sur la santé psychique et physique ainsi que leur retentissement social, familial et professionnel.

L'objet de notre travail sera, sous différents aspects, de contribuer à renforcer les éléments de connaissance de la problématique des traumatismes sexuels de l'enfance.

Dans un premier temps, nous étudierons la perception dans notre société des violences sexuelles. Nous ferons cela, par le biais de l'art, la littérature et l'histoire mais aussi l'évolution de la notion de traumatisme en psychiatrie, pour alors donner les définitions consensuelles juridiques de violences sexuelles et enfin aborder les données épidémiologiques nationales et internationales permettant d'en analyser prévalence et caractéristiques. 
Dans un second temps, et plus en pratique, nous nous intéresserons aux sujets adultes ayant été victimes de violences sexuelles. Nous tenterons d'identifier les troubles post-traumatiques, psychiques et somatiques, puis les outils dont nous disposons pour le repérage et l'évaluation de tels évènements ainsi que leurs conséquences.

Dans notre troisième partie, nous rapporterons les modalités de prises en charge existantes et recommandées, d'ordre psychothérapeutiques ou pharmacologiques, basées sur différents modèles psychopathologiques.

Après avoir posé les bases théoriques et pratiques liées aux soins de patients victimes de violences sexuelles, nous chercherons à en montrer l'aspect économique. En effet, le contexte économique actuel, pousse à l'évaluation et à la maîtrise des dépenses dans tous les domaines, la santé ne faisant pas exception. Ainsi, la quatrième partie de notre travail explicitera notre étude, son contexte et nos résultats. Plus précisément, il s'agit d'une étude pilote, testant la faisabilité d'une quantification rétrospective sur 12 mois des dépenses de santé chez des sujets adultes ayant été victimes de violences sexuelles avant l'âge de 15 ans. Les chiffres ainsi obtenus, seront-ils différents de ceux de la population générale française ?

Pour terminer notre exposé, nous illustrerons les propos précédents à l'aide de cas-cliniques, quatre patientes rencontrées au cours de notre internat, interpellant sous différents aspects notre pratique. Pour commencer, la toute première patiente qu'il nous a été donné de prendre en charge dans un service d'hospitalisation spécialisé en psychotraumatologie. La seconde aura la particularité d'être une adolescente issue de notre expérience d'un an en pédopsychiatrie. La troisième est une patiente suivie lors de nos deux passages en stage de psychotraumatologie, dont la densité des pathologies en fait un cas d'une complexité rare. La dernière quant à elle, permet de mettre en exergue les difficultés rencontrées, même au sein d'une équipe formée à cette clinique. 


\section{PREMIERE PARTIE}

\section{VIOLENCES SEXUELLES ET TRAUMATISME PSYCHIQUE, UN TOUR D'HORIZON}




\section{LES VIOLENCES SEXUELLES DANS L'HISTOIRE, L'ART ET LA LITTERATURE}

\subsection{MYTHOLOGIE GRECQUE ET EGYPTIENNE}

Le récit majeur relatant un inceste se trouve dans la mythologie grecque. Il s'agit de l'incontournable mythe d'Edipe.

Alors que Jocaste, femme de Laïos, roi de Thèbes, attend un enfant, un oracle prédit à celuici «il tuera son père; il épousera sa mère ». Une fois né, Laïos abandonne l'enfant sur le mont Cithéron. Le nouveau-né sera sauvé par des pâtres, qui le confient alors au roi de Corinthe. Polybos et sa femme l'élèveront comme leur fils. Un jour, alors qu'Oedipe est en chemin vers Thèbes pour éclaircir le mystère de sa naissance, une querelle éclate avec un homme âgé, qu'il finit par tuer. Cet homme n'était autre que Laïos son père. A l'entrée de Thèbes, Oedipe résout la fameuse énigme du sphinx, il est alors proclamé roi de Thèbes et épouse donc la reine Jocaste, qui n'est autre que sa mère biologique. Sans le vouloir il accomplit la prophétie de l'oracle. Du couple incestueux naissent quatre enfants. Lorsqu'ils prennent connaissance de la terrible vérité sur leur histoire et leur union, Jocaste se suicide et Edipe se crève les yeux.

Ce mythe sera traité par une multitude d'auteurs, peintres, sculpteurs, cinéastes et musiciens, Sophocle dans deux de ses tragédies, Edipe roi et Edipe à Colone en 400 avant J-C, Corneille en 1659, Voltaire en 1718, Gide en 1930 dans leur pièce toutes trois nommée Edipe.

Les récits de mythologie égyptienne relatent des relations incestueuses. En autres de multiples unions frère-sœur, citons simplement Osiris-Isis ou encore Seth-Nephtys. 


\subsection{L'INCESTE DANS L'HISTOIRE}

Commençons par l'Egypte Antique, avec le Grand Pharaon Ramsès II qui aurait eu des enfants avec deux de ses filles, puis Cléopâtre qui épousa deux de ses frères Ptolémée XIII et Ptolémée XIV.

Les Borgia, d'origine espagnole, se trouvent être une famille influente en Italie à la fin du Moyen-Âge et à la Renaissance. Cette dynastie est célèbre pour avoir donnée deux papes, Calixte III et Alexandre VI mais surtout pour ses mœurs scandaleuses faites de corruption, meurtres, empoisonnements, fratricides ainsi que pour les relations incestueuses entre Lucrèce Borgia, son père Rodrigo et son frère.

Cette famille a été et est encore une source d'inspiration pour de multiples romans, opéras, films, séries télévisées et même jeux vidéos et bandes dessinées. Citons Alexandre DUMAS en 1839 dans Crimes célèbres, série faite de plusieurs œuvres dont les III et IV sont Les Borgia; Michel ZEVACO dans Borgia! en 1906 et plus récemment en 2011 les séries télévisées de Tom FONTANA et Neil JORDAN.

\subsection{LE VIOL COMME ARME DE GUERRE}

Depuis l'Antiquité en passant par la Seconde Guerre mondiale, le viol a été utilisé dans les conflits comme arme de guerre et outil de répression. Instrument de torture aussi bien utilisé contre les hommes que les femmes et même les enfants. [63,213]

Les plus récents et fréquemment cités sont :

- Le génocide au Rwanda, en 1994 (500 000 viols, 70\% des victimes auraient contracté le VIH) après lequel les violences sexuelles feront partie de la définition de « génocide ».

- Le conflit bosniaque (entre 1992 et 1995, 20000 musulmanes ont été violées), le Tribunal Pénal International pour l'ex-Yougoslavie a alors retenu les qualificatifs de «torture » ainsi que de « crime contre l'humanité ». 
- En septembre 2009 à Conakry en Guinée plusieurs centaines de femmes manifestant de manière pacifique leur opposition à la dictature militaire ont été violées par les militaires du capitaine Camara.

- Début 2011 en Lybie, Kadhafi aurait « commandité des viols en masse afin de terroriser les rebelles » selon Luis Moreno Ocampo procureur de la Cour Pénale Internationale. Même scénario rapporté en Côte-d'Ivoire entre Décembre 2010 et mai 2011 entre pro-Gbagbo et pro-Ouattara.

- Encore plus récemment les témoignages et articles se multiplient rapportant « le viol comme arme en Syrie. »

Ces pratiques au moment des conflits armés, n'épargnent nullement les enfants, les adolescents et tout particulièrement les jeunes filles. La virginité pour beaucoup de cultures est un symbole sacré de pureté, la victime de viol sera alors désormais le symbole de l'humiliation et du déshonneur. Signe indélébile de dévalorisation sociale et familiale, le viol devient une atteinte à l'individu au retentissement collectif.

Ainsi, l'ONU depuis 2008 qualifie le viol de «tactique de guerre », d'autres utilisent le terme « d'arme de destruction massive ». Ces victimes devraient alors pouvoir bénéficier du statut de victime civile de guerre suite à des violences sexuelles et ainsi avoir accès à des soins adaptés.

\subsection{LES VIOLENCES SEXUELLES DANS LES ROMANS CONTEMPORAINS}

Le récit de Laurent de Villiers, Tais-toi et pardonne!, paru aux éditions Flammarion en 2011, est le témoignage des agressions sexuelles dont il dit avoir été victime par son frère [65]. Cet ouvrage relatant son parcours a trouvé un écho médiatique de part la renommée de la famille dont il est issu.

En voici quelques extraits :

«La maison est calme. Nous sommes samedi après-midi et tout le monde vaque à ses occupations. [...] Il pousse la porte du pied et entre, parle de mes GI Joe. Me demande de le suivre, on va jouer... Il a des choses à me raconter... Pour une fois qu'il est gentil, je le suis. 
Nous entrons dans sa chambre. Il est doux. Si différent. Me dit qu'il va m'expliquer plein de choses indispensables, très importantes. Comme le sexe. Il me dit que nous allons jouer et que ça va être agréable. Il me dit que je suis son petit frère, qu'il m'aime. [...] Il me dit que nous sommes pareils, que nous avons le même problème, que nous sommes pervers, obsédés par le péché de chair... Je ne comprends pas grand-chose. [...] Il me dit que nous " jouons ", mais qu'il ne faut pas en parler. Pendant six mois, Guillaume vient me chercher régulièrement dans ma chambre. [...] Souvent, j'arrive à table en pleurs d'avoir croisé mon frère quelques instants auparavant. Alors maman me demande ce que j'ai et je n'ose pas lever les yeux. Il guette. "Rien, rien... Je suis tombé dans les escaliers." Guillaume me fait peur. Il m'a toujours fait peur. Lui n'a peur de rien. Maman m'aide à ne rien dire. Quand elle remarque des bleus, elle hausse les épaules. "Ah, mais tu ne peux pas faire attention !" ou " C'est le petit Jésus qui t'a puni. " Puni de quoi ? » p99-101

«Nous sommes en juin 2002 et je suis seul à la maison. [...] J'ai ouvert une bouteille de vodka et déjà descendu quelques verres. Sans même réfléchir, comme une évidence absolue, je me dirige vers la chambre de papa et maman. Je n'ai même pas à chercher. [...] J'ouvre la penderie. Je pousse la rangée de costumes de papa. Au fond, posé dans sa housse de cuir, le fusil m'attend. [...] Je suis calme, le fusil entre mes mains. Je sais ce qu'il me reste à faire. Je regarde le papier sur lequel j'ai griffonné à l'intention de papa, maman, mes frères et sœurs, un seul mot : Pourquoi ? J'enlève la sécurité. J'arme. Je positionne le canon du fusil face à mon visage, entre mes yeux. Ça devrait être rapide. Je pose mon doigt sur la détente. J'inspire avant le grand saut. J'expire, prêt à tirer. Mais dans le silence absolu de la maison, une sonnerie retentit. Mon portable. Je pensais l'avoir éteint. Le nom de Benoît apparaît sur l'écran illuminé. Mécaniquement, je décroche. Nous parlons, de tout, de rien, comme d'habitude. [...] Merci Benoît. »p147-150

«Depuis, j'ai enfin trouvé la force de parler. De les affronter, de combattre leur silence aujourd'hui devenu mensonge. Je n'en peux plus de me taire. Je n'en peux plus d'avoir mal. Il faut qu'ils sachent. Il faut que tout le monde sache. » 219

« Mais ce jour là, pour la première fois, je décris véritablement les images qui me hantent, ces scènes que j'ai rejetées [...] J'ai honte. Je me sens coupable de porter plainte, d'être obligé de porter plainte. Je porte en moi la honte tenace d'avoir été souillé, sali. La honte, aussi, de ne pas avoir pu ni su me défendre, de ne pas avoir su ni pu l'empêcher. Dix ans après, j'en suis encore l'acteur, puisque assis sur une chaise à raconter à l'inspectrice ce que j'ai subi. Et je dois répondre, encore et toujours, à la question que je ne cesse de me poser : " Pourquoi vous 
n'avez rien fait ? " Pourquoi ?... Parce que j'avais 10 ans, parce qu'il en avait 16... Parce que je n'avais ni la maturité intellectuelle ni la force physique. Des mots et une masse informe de culpabilité. »p225-226

«J'ai toujours cru à la guérison par la vie. Pour qu'un enfant blessé devienne un homme, il faut qu'il redécouvre son enfance et accepte de ne pas en avoir eu. Il m'aura fallu attendre de rencontrer ma fille pour comprendre ce qu'est la vie. Avec ses premiers pas, je refais les miens. » $p 273$

«Dans quelques mois, je vais devenir citoyen américain. Je choisis le pays des rêves, de la renaissance et renonce à tout jamais à mon nom. [...] Aujourd'hui, je ne suis plus le fils de personne. »p282

Autre récit autobiographique, celui de Margaux Fragoso, Tigre, tigre !, paru en 2012 chez Flammarion. Margaux, dont la mère est bipolaire et le père alcoolique violent, grandit au sein de cette famille défaillante. Vers l'âge de 7ans, elle croise à la piscine communale, Peter, 51 ans. Cet homme s'immisce alors dans la famille, prenant l'apparente place d'un tuteur pour Margaux, il est en réalité un pédophile qui sera son agresseur pendant 14 ans. Sur la préface du roman on peut lire: «Lyrique, profond et d'une limpidité hypnotique, Tigre, tigre! dépeint d'une manière saisissante les forces opposées de l'emprise et de la mémoire, de l'aveu et du déni, et questionne nos capacités de guérison. Un récit extraordinaire qui dévoile de l'intérieur la pensée d'une jeune fille au bord de la chute libre. » [97]

Christine Angot quant à elle, s'est illustrée à deux reprises sur ce thème, avec Inceste paru chez Stock en 1999, puis en 2012 avec Une semaine de vacances paru chez Flammarion.

Les violences sexuelles demeurent un thème embarrassant dans la littérature, souvent abordées sans concessions, les publications défrayent alors la chronique. Déjà en 1955 le roman Lolita de Vladimir Nabokov avait fait scandale. Ce récit dérangeant, dont le narrateur Humbert Humbert relate sa relation pédophile avec une jeune fille de 12 ans, sera adapté au cinéma en 1962 par Stanley Kubrick. 


\section{HISTORIQUE DE LA NOTION DE TRAUMA EN PSYCHIATRIE : DE L'HYSTERIE A L'ETAT DE STRESS POST-TRAUMATIQUE}

Passer en revue l'évolution de la notion de traumatisme en psychiatrie nécessite dans un premier temps d'exposer son lien avec l'hystérie, pour mieux s'en détacher par la suite.

\subsection{HYSTERIE ET TRAUMATISME}

Dans l'Egypte ancienne, les troubles se rapportant à ce que l'on appelle aujourd'hui hystérie étaient associés «à la migration de l'utérus dans le haut du corps ». Théorie reprise par Hippocrate dans le traité Des maladies de femmes et donne le nom d'hystérie à ses manifestations en lien avec « hystera », utérus en grec.

Plus tard, d'autres se sont attachés à réfuter la théorie utérine, comme Charles le Pois (15631633), Thomas Willis (1621-1675) défendant la thèse de l'origine cérébrale de cette affection car pouvant toucher les deux sexes. Thomas Sydenham (1624-1689) lui, y voit « un désordre ou mouvement irrégulier des esprits animaux » et fait l'ébauche de la description de la personnalité hystérique.

Le terme de syndrome de Briquet a été utilisé dans certaines anciennes classifications pour nommer l'hystérie. En effet, Paul Briquet (1796-1881), médecin français à l'hôpital de la Charité, marque une véritable avancée avec son Traité clinique et thérapeutique de l'hystérie paru en 1859. Il y regroupe 430 observations cliniques d'hystérie lui permettant de fournir hypothèses étiologiques et données épidémiologiques. Associant symptômes physiques et psychiques il définit l'hystérie comme «une névrose de l'encéphale, dont les phénomènes apparents consistent principalement dans la perturbation des actes vitaux qui servent à la manifestation des sensations affectives des passions. » (Garrabé Jean, 2009) [105]

«Briquet pense que le siège de l'hystérie se trouve dans ce qu'il appelle "la partie affective de l'encéphale" et que cette partie est distincte de "la partie intellectuelle" du cerveau. [...] La "partie affective" de Briquet a été appelée "le cerveau viscéral" par Papez en 1938 et est connu actuellement sous le nom de "système limbique", imaginé par Maclean en 1949. » (Mai F. 1980) [155]. 
Ainsi, il est l'un des premiers à distinguer l'émotionnel du cognitif et pour qui l'hystérie traduit alors une souffrance de l'ensemble du corps. Sur le plan épidémiologique, il retrouve un cas d'hystérie masculine pour 20 cas d'hystérie féminine. 365 sujets présentaient un évènement pouvant constituer un choc émotionnel dans leur histoire (deuil, viol, maltraitance). Selon lui quasiment $50 \%$ de ces sujets de moins de 12 ans avaient été l'objet de mauvais traitements. (Darves-Bornoz 1996) [52]

J.M Charcot (1825-1892), neurologue à la Pitié-Salpêtrière, conserve l'idée de l'origine cérébrale et non utérine de l'hystérie. Charcot souhaite prouver l'étiologie organique de l'hystérie, espoir qui restera vain. En effet, il utilise l'hypnose lors de ses fameuses leçons du mardi, pour provoquer les symptômes de l'hystérie telles que «les paralysies hystériques » et met ainsi en évidence leur base psychogène et non neurologique pure. Il redonne sa place de maladie à l'hystérie et la distingue des simulations ainsi que des autres maladies neurologiques. Il va de plus se pencher sur la cause traumatique dans les leçons 18 à 22, rapportant sept cas d'hystérie masculine, utilisant le terme «d'hystérie traumatique ». Il introduit ainsi la notion de dissociation de la conscience dans les suites de «choc nerveux » traumatiques. Sujet repris et développé par P. Janet et S. Freud.

\subsection{CONCEPTION JANETIENNE ET PSYCHOPATHOLOGIE DES TRAUMATISMES PSYCHIQUES}

Pierre Janet (1859-1947), philosophe et psychologue français, sera nommé directeur du Laboratoire de psychologie expérimentale de la Salpêtrière par Charcot, puis exercera comme professeur au Collège de France.

Il effectue des travaux basés sur l'observation de sujets à travers «les actes, les gestes, le langage» et introduit avec les cas Léonie et Lucie, la notion «d'automatisme psychologique ». Théorie qu'il expose dans sa thèse de Lettres en 1889, L'automatisme psychologique. Essaie de psychologie expérimentale sur les formes inférieures de l'activité humaine. Il définit comme activité «subconsciente », l'automatisme psychologique : «une forme rudimentaire de la conscience où l'activité, la sensibilité et l'intelligence se confondent absolument ». Il en distingue deux types, le total, l'esprit étant alors totalement dominé par la 
reproduction de l'expérience passée, se manifestant par la catalepsie et le partiel, au sein duquel on retrouve entre autre la catalepsie partielle, le phénomène d'anesthésie, l'amnésie post hypnotique, le somnambulisme, les existences psychologiques simultanées et successives, les sensations d'étrangeté, le spiritisme, le médiumnisme, les tics ou encore l'écriture automatique. Il y affirme donc une présence de la conscience, même élémentaire: l'automatisme psychologique «est un phénomène psychologique autonome, comportant toujours une conscience rudimentaire $»$ (Janet Pierre, 1889) [128].

Dans sa thèse, il développe aussi la notion de «désagrégation mentale », équivalente de la « dissociation », terme qu'il n'utilisera que plus tard. Il s'agit d'un « rétrécissement du champ de la conscience », certaines informations sont enregistrées par la conscience alors que les autres ne sont prises en compte que par le subconscient perturbant la capacité de synthèse. Cette dissociation des informations, peut être provoquée par une «fatigue d'origine normale ou pathologique » pouvant faire référence à de fortes émotions ou à un épuisement. On peut en déduire alors facilement que les traumatismes psychologiques complexes, peuvent aboutir aux troubles dissociatifs. (Kédia 2009) [134].

Il est important de différencier la dissociation dont parle Janet, proche mais non équivalente $\mathrm{du}$ «Spaltung» de Bleuler traduit aussi en français par dissociation mais signifiant discordance et clivage du moi dans le cadre des troubles psychotiques.

Ses études de cas lui permettent d'envisager comme traitement des troubles dissociatifs les méthodes cathartiques. L'observation de la jeune Marie en est une bonne illustration :

«Cette jeune fille fut amenée de la campagne à l'hôpital du Havre à l'âge de 19 ans, parce qu'on la considérait comme folle et qu'on désespérait presque de sa guérison. En réalité elle avait des périodes de crises convulsives et de délires qui duraient des journées entières. Après quelque temps d'observation, il était facile de constater que la maladie se composait d'accidents périodiques, revenant régulièrement au moments de ses époques et d'autres accidents moins graves se prolongeant et survenant irrégulièrement dans ses intervalles [...] Je voulus avoir des renseignements précis sur la façon dont ses époques avaient commencé et comment elles avaient été interrompues. Elle ne répondit pas clairement, car elle paraissait avoir oublié une grande partie des choses qu'on lui demandait. Je songeai alors à la mettre dans un somnambulisme profond, capable, comme on l'a vu, de ramener des souvenirs en apparence oubliés et pus ainsi retrouver la mémoire exacte d'une scène qui n'avait jamais été connue que très incomplètement. A l'âge de 13 ans, elle avait été réglée pour la première fois, 
mais par la suite d'une idée enfantine et d'un propos entendu et mal compris, elle se mit en tête qu'il y avait quelque honte et chercha le moyen d'arrêter l'écoulement. Vingt heures à peu près après le début, elle sortit en cachette et alla se plonger dans un grand baquet d'eau froide. Le succès fut complet, les règles furent arrêtées subitement et malgré un grand frisson qui survint, elle put rentrer chez elle. Elle fut malade assez longtemps et eut plusieurs jours de délire $[\ldots]$

Quand elles ont réapparu elles ont amené les troubles que j'ai observés. Or si l'on compare l'arrêt subit, le frisson, les douleurs qu'elle décrit aujourd'hui en état de veille avec le récit qu'elle fait en somnambulisme et qui d'ailleurs a été confirmé indirectement, on arrive à cette conclusion : tous les mois, la scène du bain froid se répète avec le même arrêt des règles et un délire qui est, il est vrai beaucoup plus fort qu'autrefois [...] Mais dans la conscience normale, elle ne sait rien de tout cela et ne comprend même pas que le frisson est amené par l'hallucination du froid ; il est donc vraisemblable que cette scène se passe au dessous de cette conscience et amène tous les autres troubles par contre coup [...]

Je ne pus réussir à effacer cette idée que par un singulier moyen. Il fallut la ramener à l'âge de 13 ans, la remettre dans les conditions initiales du délire, et alors la convaincre que les règles avaient durée trois jours et n'avait été interrompues par aucun accident fâcheux. Eh bien, cela fait, l'époque suivante arriva à sa date et se prolongea pendant trois jours, sans amener aucune souffrance, aucune convulsion, ni aucun délire [...]

Voilà cinq mois que ses expériences ont été faites, Marie n'a plus présenté le plus léger signe d'hystérie, elle se porte fort bien et surtout se renforce beaucoup. Son aspect physique a absolument changé. Je n'attache pas à cette guérison plus d'importance qu'elle n'en mérite, et je ne sais pas combien de temps elle durera, mais j'ai trouvé cette histoire intéressante pour montrer l'importance des idées fixes subconscientes et le rôle qu'elles jouent dans certaines maladies physiques aussi bien que dans les maladies morales. » (Janet Pierre, 1889) [128].

Les bases de ce qu'il nomme la «psychologie dynamique » sont alors posées, théorie de nos jours réactualisée par les travaux en neurosciences de Onno Van Der Hart et Ellert Nijenhuis aux Pays-Bas ainsi que ceux de Kathy Steele aux Etats-Unis.

Les écrits de Janet n'attachent pas d'importance particulière à la sexualité comme origine des névroses et donc ne mettent pas particulièrement en avant les traumatismes d'ordre sexuel. C'est entre autres en cela, que sa théorie sera perçue comme concurrente à celle de son contemporain Sigmund Freud. 


\subsection{FREUD : DE LA « NEUROTICA », THEORIE DE LA SEDUCTION OU DU TRAUMATISME SEXUEL, A LA THEORIE DU FANTASME OU DE LA SEXUALITE INFANTILE}

Sigmund Freud (1856-1939), neurologue viennois, intègre au début de son parcours en 1885, l'équipe de Charcot à la Salpêtrière. Quatre mois à s'imprégner des idées de Charcot, « un des plus grands médecins dont la raison confine au génie [...] personne n'a jamais eu autant d'influence sur moi »

Dans la continuité, il élaborera sa première théorie des névroses : «Chaque fois qu'une contrainte névrotique survient dans le psychique, elle provient du refoulement ». [102] Alors que selon Janet la dissociation résulte d'un déficit fonctionnel, pour Freud il s'agit d'un mécanisme de défense protégeant la conscience. Sa collaboration avec Josef Breuer, dont l'étude du cas Anna O, sera marquée par la publication en 1895 de Les Etudes sur l'Hystérie. Ils mettent alors en exergue le lien entre traumatisme, dissociation et hystérie: «Nos observation montrent qu'un traumatisme grave (comme celui d'une névrose traumatique), une répression pénible (celle de l'affect sexuel, par exemple) peuvent provoquer, même chez un sujet normal, une dissociation des groupes de représentations et c'est en cela que consisterait le mécanisme de l'hystérie psychiquement acquise ». La théorie de la séduction définit alors comme origine traumatique de la névrose hystérique une maltraitance sexuelle vécue dans l'enfance.

L'année 1896 marque pour Freud le décès de son père et son repositionnement théorique basé sur la structure œdipienne. En témoigne une lettre adressée à son ami W. Fliess en septembre 1897, dans laquelle il écrit : «Je ne crois plus à ma neurotica [...] dans chacun des cas il fallait accuser le père, et ceci sans exclure le mien de perversion, [...] une telle généralisation de ces actes envers des enfants semble peu croyable [...] il existe aucun indice de réalité dans l'inconscient de telle sorte qu'il est impossible de distinguer la vérité et la fiction investie d'affect. » [101].

Le désaveu sera révélé en 1906 dans Trois Essais sur la théorie sexuelle. Il abandonne donc son premier postulat selon lequel les névroses auraient pour substrat un traumatisme, par exemple une agression sexuelle par un adulte, le plus souvent le père pour les patientes hystériques, mais selon sa nouvelle théorie, un désir réprimé. Les scènes traumatiques sont alors de l'ordre du fantasme inconscient.

Ce positionnement de Freud, père de la psychanalyse, aura pour conséquence pratique de négliger les traumas réels au profit de la théorie du fantasme. 


\subsection{FERENCZI, L'ENFANT TERRIBLE DE LA PSYCHANALYSE}

Sandor Ferenczi (1873-1933), psychanalyste hongrois, à ses débuts disciple de Freud, se détachera au fil de sa pratique de la pensée Freudienne, remettant en cause la théorie du fantasme.

Ainsi, en 1932, avec sa publication Confusion de langue entre les adultes et l'enfant. Le langage de la tendresse et de la passion, relatant des traumatismes pour la plupart d'ordre sexuel, il redonne sa place au traumatisme dans la genèse de certains troubles mentaux. «On ne pourra jamais insister assez sur l'importance du traumatisme et en particulier du traumatisme sexuel comme facteur pathogène. Même des enfants appartenant à des familles honorables [...] sont des victimes de violences et de viols [...] L'objection qu'il s'agissait de fantasmes de l'enfant lui-même, c'est-à-dire de mensonges hystériques, perd malheureusement de sa force par suite du nombre considérable de patients en analyse qui avouent eux-mêmes des voies de fait sur des enfants ». [80]

Il s'agit là de l'adulte répondant de manière sexuée et non adaptée au besoin de tendresse de l'enfant. Celui ci, n'étant pas mature et n'ayant pas le matériel psychique pour l'élaborer sera sujet à des troubles psychiques dont un clivage du moi.

Il introduit alors la notion «d'identification à l'agresseur » comme un des mécanismes de défense de la victime. Il souligne de plus comme facteur aggravant le déni de l'adulte ne reconnaissant pas la souffrance de l'enfant.

En termes de pratique, il remet en question le principe de «neutralité bienveillante » du psychanalyste pour remanier à plusieurs reprises la sienne, passant de sa «Technique active » à «l'analyse mutuelle», en passant par «l'élasticité technique », utilisant néocatharcis et relaxation.

Certaines idées défendues par Ferenczi, peuvent être retrouvées dans les travaux de Mélanie Klein et Donald Winnicott entre autres. 


\subsection{LES GUERRES DU XX ${ }^{\mathrm{e}}$ SIECLE, LA NEVROSE DE GUERRE ET L'ETAT DE STRESS POST-TRAUMATIQUE}

Au milieu des conflits théoriques exposés précédemment, deux personnages se doivent d'être cités :

- Herman Oppenheim (1857-1919), neurologue allemand, est le premier à utiliser le terme de « Névrose traumatique » en 1889 pour décrire les symptômes présentés par les victimes d'accident de chemin de fer. Il rapporte alors des changements organiques chez des victimes de trauma psychologiques, par exemple des maladies cardiovasculaires chez des soldats.

- Charles Samuel Myers (1873-1946), médecin anglais et consultant psychologue pour la British Army au cours de la première guerre mondiale, utilisa le terme de « shell shock » ou « choc des tranchées » dans l'un de ses articles publiés en 1915 dans The Lancet.

Malgré son désaveu de la Neurotica, Freud n'a pas pour autant complètement abandonné la notion de traumatisme. En témoigne Au-delà du principe de plaisir en 1920, en partie écrit sur son travail avec des soldats survivant de la première guerre. Il ne sera pas le seul en ces temps d'après guerre à s'exprimer sur ce thème. Un ouvrage reprend son intervention, ainsi que celles de $\mathrm{S}$. Ferenczi et $\mathrm{K}$. Abraham, au $\mathrm{V}^{\mathrm{ème}}$ Congrès International de psychanalyse à Budapest, automne 1918, Sur les névroses de guerre. Dans l'introduction faite par Freud on peut lire : «Les névroses de guerre, pour autant qu'elles se distinguent de névroses banales du temps de paix par des propriétés particulières, sont à concevoir comme des névroses traumatiques qui ont été rendues possibles ou qui ont été favorisées par un conflit du moi. » (Freud S., 1919/1984) [100]

Il faut attendre la fin de la Seconde Guerre mondiale, ses survivants des camps de concentration et ses anciens combattants, pour voir un nouvel élan permettant une avancée sur le trauma. Ainsi, deux psychanalystes américains, Roy Grinker et John Spiegel publièrent en 1945 leurs études cliniques de pilotes de l'armée de l'air, Men Under Stress. Ils exposent entre autre, l'importance du soutien moral du groupe, pour affronter et surmonter le stress au combat. Henry Krystal, né en 1925 en Pologne, survivant de l'Holocauste immigrera en 1947 à Détroit aux Etats-Unis, où il deviendra psychiatre. Il travaillera tout naturellement alors sur les survivants des camps et décrira les prémices de la notion actuelle d'alexithymie. 
La problématique des agressions sexuelles dont le viol, alors délaissée en temps de guerre, sera replacée au sein du traumatisme psychique, par deux américaines, Ann Burrgess, psychiatre et Linda Holmstrom, sociologue. Elles sont les premières, en 1974 à décrire le « rape trauma syndrom » ou «syndrome traumatique du viol», le comparant au traumatisme de guerre. Selon elles, la réaction au viol comprend deux phases. La première, «phase aiguë», de désorganisation, se manifestant par des «réactions de choc» ainsi que des «réactions somatiques ». La seconde, «phase de réorganisation » ou encore de modification du mode de vie.

Parallèlement, en France, les travaux sur le trauma psychologique seront restreints et uniquement marqués dans les années 70 par ceux de deux psychiatres militaires, Louis Crocq et Claude Barrois. Depuis et sous leur impulsion, la psychotraumatologie en France s'est développée, aussi bien dans l'enseignement, grâce à la mise en place de formations et diplômes spécifiques, que dans la pratique avec la création des CUMP (Cellule d'Urgence Médico-Psychologique). La circulaire du 28 mai 1997 relative à la création d'un réseau national de prise en charge de l'urgence médico psychologique en cas de catastrophe, fait suite à l'attentat terroriste en juillet 1995 de la station de RER Saint-Michel à Paris.

C'est à la suite de la guerre du Vietnam (1964-1975), que le terme de «trouble de stress post traumatique » se développera progressivement. Les Etats-Unis montrant un regain d'intérêt pour les troubles présentés par ses vétérans du Vietnam. Le PTSD (Post-Traumatic Stress Disorder) en français ESPT (Etat de Stress Post-Traumatique) n'aura sa définition rigoureuse qu'en 1980, lors de sa réintroduction au sein de la catégorie des troubles anxieux dans le Manuel diagnostique et statistique des troubles mentaux-troisième révision (DSM-III), élaboré par la Société Américaine de Psychiatrie, sous la direction de Robert Spitzer pour cette édition. L'ESPT ayant été auparavant inscrit au DSM-I en 1952 mais supprimé du DSMII en 1968. En 1992, il sera inclu à la $10^{\text {ème }}$ édition de la Classification internationale maladie (CIM 10) de l'Organisation Mondiale de la Santé, puis en 1994, le DSM-IV intégrera la notion d'état de stress aigu. 


\section{COMMENT DEFINIR LES VIOLENCES SEXUELLES ?}

En 2002, selon l'Organisation Mondiale de la Santé (OMS), le terme de violence sexuelle regroupe «Tout acte sexuel, tentative pour obtenir un acte sexuel, commentaire ou avances de nature sexuelle, ou actes visant à un trafic ou autrement dirigés contre la sexualité d'une personne utilisant la coercition, commis par une personne indépendamment de sa relation avec la victime, dans tout contexte, y compris mais sans s'y limiter, le foyer et le travail »

On constate alors que derrière la notion de violence sexuelle, il existe plusieurs entités qui peuvent être classées de diverses façons. Utiliser le cadre juridique français peut nous permettre de les distinguer.

Les articles de loi sont consultables sur : http://www.legifrance.gouv.fr [147]

Loin d'ignorer les limites de ces définitions, elles ont le mérite d'éviter toutes appréciations subjectives et sont nécessaires au principe de légalité, notamment en droit pénal.

\subsection{MAJORITE SEXUELLE EN FRANCE}

Il est nécessaire avant de classifier les violences sexuelles, de définir la notion de majorité sexuelle ou plutôt «d'âge de consentement». Il s'agit pour la loi, de «l'âge minimum que doit avoir une personne pour avoir des relations de son plein gré » ou encore « de l'âge à partir duquel un mineur civil peut entretenir une relation sexuelle avec un adulte sans que cet adulte ne commette une infraction pénalement réprimée. »

En France, l'article 227-25 du code pénal fixe la majorité sexuelle à 15 ans depuis 1945. A noter qu'il varie d'un pays à l'autre, entre 11 et 21 ans. Ainsi, au Vatican il est de 12 ans alors qu'à Malte il est de 18 ans. 


\subsection{CADRE JURIDIQUE DES VIOLENCES SEXUELLES}

Les violences sexuelles sont regroupées en deux grandes classes, les agressions sexuelles (viol, attouchement, exhibitions sexuelles) puis les autres violences sexuelles (atteintes sexuelles sur mineur, harcèlement sexuel...)

\subsubsection{Les agressions sexuelles}

L'article 222-22 du code pénal définit comme agression sexuelle, «toute atteinte sexuelle commise avec violence, contrainte, menace ou surprise ». Définition qui comprend le viol ainsi que les agressions sexuelles autres que le viol.

\subsubsection{Le viol}

Selon l'OMS (2002), le viol est un «acte de pénétration, même légère, de la vulve ou de l'anus imposé notamment par la force physique en utilisant un pénis, d'autres parties du corps ou un objet. Il y a tentative de viol si l'on essaie de commettre un tel acte. La violence sexuelle peut comprendre d'autres formes d'agression dans lesquelles intervient un organe sexuel notamment le contact imposé entre la bouche et le pénis, la vulve ou l'anus. Lorsqu'il y a viol d'une personne par deux ou plusieurs agresseurs, on parle de viol collectif. »

Le viol est la plus grave des agressions sexuelles dans le domaine juridique, qualifié de crime et définit dans les articles 222-23 à 222-26 du code pénal comme «tout acte de pénétration sexuelle de quelque nature que ce soit commis sur la personne d'autrui par violence, contrainte, menace ou surprise ». L'auteur encourt alors une peine de 15 ans de réclusion criminelle et jusqu'à 20 ans en cas de circonstance aggravante.

Une circonstance aggravante peut être l'âge de la victime. En effet, un rapport sexuel avec un mineur de moins de 15 ans est toujours qualifié de viol. Autre circonstance aggravante, si l'auteur est « un ascendant légitime naturel ou adoptif ou par toute personne ayant autorité sur la victime » ou qui «abuse de l'autorité que lui confère ses fonctions ». Cette définition permet de prendre en compte les cas ou les faits seraient commis par le père, un grand-père, un beau père, un oncle, un enseignant, un moniteur ou éducateur mais aussi un ami de famille ou voisin ayant autorité sur l'enfant. 


\subsubsection{Les agressions sexuelles autres que le viol}

Les agressions sexuelles autres que le viol se retrouvent dans les articles 222-27 à 222-31 du code pénal.

Elles regroupent «tous les faits d'attouchements sexuels commis avec violence, contrainte, menace ou surprise sans acte de pénétration sexuelle ». L'auteur encourt alors 5 ans de prison et 75000 euros d'amende.

Dans le cas des agressions sexuelles autres que le viol, deux catégories de circonstances aggravantes peuvent être retenues :

- Les faits imposés à un mineur de 15 ans ou à une personne particulièrement vulnérable. La peine peut être de 7 ans de prison et 100000 euros d'amende.

- Les faits imposés à un mineur de 15 ans par plusieurs personnes, ainsi que les faits imposés par un ascendant légitime, naturel ou adoptif ou par une personne ayant abusé de l'autorité que lui confèrent ses fonctions. La peine peut être de 10 ans de prison et 150000 euros d'amende.

La loi ne fait donc pas de distinction en termes de condamnation « entre les agressions commises par un membre de la famille, par une personne de la communauté scolaire ou éducative ou par un groupe de jeunes sur un mineur de moins de 15 ans (racket sexuel) »

\subsubsection{L'exhibition sexuelle}

L'exhibition sexuelle est aussi inscrite au code pénal (article 222-32), faisant partie des agressions sexuelles. Elle n'est condamnable que si «imposée à la vue d'autrui, elle a lieu dans un endroit accessible au regard du public ». L'auteur peut être puni d'un an de prison et de 15000 euros d'amende. 


\subsubsection{Les autres violences sexuelles}

\subsubsection{Les atteintes sexuelles sur mineur}

Définis dans l'article 227-25 du code pénal comme étant tous «attouchements sexuels commis par un majeur sur un mineur de 15 ans, sans violence contrainte menace ni surprise ». Est donc considéré comme délit, toute relation sexuelle, y compris consentie entre un majeur et un mineur sexuel. L'auteur encourt 5 ans de prison et 75000 euros d'amende.

Les circonstances aggravantes pouvant être retenues se trouvent dans l'article 227-26 du Code Pénal et sont :

- si l'auteur est « un ascendant ou une personne ayant autorité sur la victime »

- si l'auteur est « une personne abusant de l'autorité que lui confère ses fonctions »

- si le délit a été commis par «plusieurs personnes, auteurs ou complices »

- «si la victime a été mise en contact avec l'auteur grâce à l'utilisation, pour la diffusion de messages à destination d'un public non déterminé, d'un réseau de télécommunications » (loi $\mathrm{n}^{\circ} 98-468$ du 17 juin 1998)

La peine pourra alors être de 10 ans d'emprisonnement et 150000 euros d'amende.

L'article 227-27 du code pénal, définit quant à lui le cas ou l'atteinte sexuelle a été commise sur un sujet de plus de 15 ans, mais moins de 18 et non émancipé par le mariage. L'auteur doit être un ascendant, une personne ayant autorité ou encore une personne abusant de l'autorité que lui confère ses fonctions. La condamnation peut être de 2 ans de prison et 30000 euros d'amende. Dans les autres cas le mineur étant considéré en âge de consentir, la loi ne retient pas d'infraction.

\subsubsection{Le proxénétisme}

« Activité délictueuse consistant à favoriser la prostitution ou à en tirer profit. » (Définition du Larousse).

En France, il est considéré comme une atteinte à la dignité de la personne. Il est puni de 7 ans d'emprisonnement et de 150000 euros d'amende. 
L'article 225-5 du code pénal le définit comme «le fait, par quiconque, de quelque manière que ce soit : d'aider, d'assister ou de protéger la prostitution d'autrui ; de tirer profit de la prostitution d'autrui, d'en partager les produits ou de recevoir des subsides d'une personnes se livrant habituellement à la prostitution; d'embaucher, d'entrainer ou de détourner une personne en vue de la prostitution ou d'exercer sur elle une pression pour qu'elle se prostitue ou continue à le faire. »

\subsubsection{Deux notions qui font débat sur le plan juridique}

\subsubsection{Le harcèlement sexuel}

Le harcèlement sexuel a été introduit comme délit en France dans le code pénal en 1992 (précisé en 1998) par l'article 222-23 comme étant «le fait de harceler autrui en donnant des ordres, proférant des menaces, imposant des contraintes ou exerçant des pressions graves dans le but d'obtenir des faveurs de nature sexuelle, par une personne abusant de l'autorité que lui confère ses fonctions. » La peine est alors d'un an de prison et 15000 euros d'amende.

La loi du 17 janvier 2002 a modifié sa définition dans le but de la clarifier et d'élargir son champ d'application devenant alors «le fait de harceler autrui dans le but d'obtenir des faveurs de nature sexuelle ». La difficulté réside alors pour la majorité des instructions, dans l'absence de preuves matérielles. Cependant, après 2003, la victime n'a plus à établir la réalité de son absence de consentement alors que l'auteur, lui, doit apporter des éléments justifiant son comportement. A partir de 2007, un SMS envoyé par le harceleur peut être reconnu comme élément de réalité des faits.

Le 4 mai 2012, le conseil constitutionnel abroge l'article, le considérant trop imprécis. Le harcèlement sexuel n'est alors plus considéré comme une infraction. De nombreuses associations, personnalités publiques et politiques s'exprimeront et manifesteront face à ce vide juridique.

Le 6 août 2012, la loi précisera l'article 222-33 du code pénal définissant alors comme délit de harcèlement sexuel : «Le fait d'imposer à une personne, de façon répétée, des propos ou comportement à connotation sexuelle qui, soit portent atteinte à sa dignité en raison de leur caractère dégradant ou humiliant, soit créent à son encontre une situation intimidante, hostile 
ou offensante. Est assimilé au harcèlement sexuel le fait, même non répété d'user de toute forme de pression grave dans le but réel ou apparent d'obtenir un acte de nature sexuelle, que celui-ci soit recherché au profit de l'auteur des faits ou au profit d'un tiers »

La peine pouvant être encourue est de 2 ans de prison et 30000 euros d'amende.

Là encore, elle peut être majorée à 3 ans d'emprisonnement et 45000 euros d'amende en cas de circonstances comprenant « les faits commis :

- Par une personne abusant de l'autorité que lui confère ses fonctions sur mineur de 15 ans.

- Sur une personne dont la particulière vulnérabilité, due à son âge, à une maladie, à une infirmité, à une déficience physique ou psychique ou à un état de grossesse, est apparente ou connue de leur auteur.

- Sur une personne dont la particulière vulnérabilité ou dépendance résultant de la précarité de sa situation économique ou sociale est apparente ou connue de leur auteur.

- Par plusieurs personnes agissant en qualité d'auteur ou de complice. »

\subsubsection{L'inceste}

La loi du 8 février 2010 inscrivait pour la première fois la notion d'inceste au code pénal avec l'article 222-32-1 : «Les viols et les agressions sexuelles sont qualifiés d'incestueux lorsqu'ils sont commis au sein de la famille sur la personne d'un mineur par un ascendant, un frère, une sœur ou par toute personne, y compris s'il s'agit d'un concubin d'un membre de la famille, ayant sur la victime une autorité de droit ou de fait. »

Le conseil constitutionnel a censuré cette loi en septembre 2011, considérant la définition de «membre de la famille » trop imprécise et donc contraire à la constitution. Aujourd'hui, dans le domaine pénal, l'inceste est indirectement pris en compte au sein des crimes et délits sexuels avec circonstance aggravante, c'est-à-dire commis par « un ascendant légitime naturel ou adoptif ou toute personne ayant autorité sur la victime. »

On doit donc à ce jour se tourner vers les dictionnaires : «Union illicite entre des personnes liées par un degré de parenté entraînant la prohibition du mariage » (Petit Larousse). Définition qui se rapproche de la notion des articles 161 à 164 du code civil et ce depuis 1804 : «En ligne directe, le mariage est prohibé entre tous les ascendants et descendants et les alliés dans la même ligne ». 


\subsection{DELAI DE PRESCRIPTION}

Dans le cas d'agression sexuelle (article 8 du code de procédure pénale) :

- Si la victime est majeure au moment des faits, le délai est de trois ans, à compter de la date des faits.

- Si la victime est mineure au moment des faits, le délai est alors de dix ans à compter de la majorité.

- Lorsqu'il y a circonstance aggravante, le délai est alors de vingt ans.

En cas de viol (article 7 du code de procédure pénale) :

- Si la victime est majeure au moment des faits, le délai est de dix ans, à compter de la date des faits.

- Si la victime est mineure au moment des faits, le délai est alors de vingt ans. 


\section{EPIDEMIOLOGIE DES VIOLENCES SEXUELLES}

Les données épidémiologiques concernant les violences sexuelles, notamment lorsque les victimes sont mineures, sont issues de diverses études et rapports, de méthodologies variables, de part les définitions et législations non consensuelles au niveau international. Cependant, elles ne nous fournissent pas uniquement des chiffres de prévalence et d'incidence, elles nous permettent aussi des caractériser globalement les victimes, les agresseurs et les types de violence. Elles attirent de plus notre attention, sur les éventuels facteurs de risque et séquelles de violences sexuelles dans l'enfance.

Nous allons passer en revue les publications majeures, aussi bien en France qu'à l'étranger, afin d'obtenir un panorama du sujet dans le monde occidental.

\subsection{DONNEES INTERNATIONALES}

Au Canada, le rapport du comité Badgley (1984) est la référence dans le domaine des agressions sexuelles ainsi que des violences à l'endroit des jeunes [9]. Il s'agit d'un sondage national, réalisé au sein d'un échantillon d'adultes. Leurs résultats montrent que :

- Une femme sur deux et un homme sur trois, ont été victimes de plusieurs actes sexuels non désirés.

- 4\% de ces actes ont été commis pendant l'enfance et l'adolescence.

- 4\% de ces femmes ont été violées, $2 \%$ des hommes ont été l'objet de tentatives ou d'acte de pénétrations.

- 1 agresseur sur 4 est un membre de la famille ou une personne de confiance et 1 agresseur sur 6 était un étranger.

- Au sein de la famille, les filles sont davantage que les garçons, victimes de violences sexuelles.

- L'âge médian au moment des faits est entre 8 et 10 ans.

- $1 \%$ des agresseurs sont de sexe féminin.

- Moins de $28 \%$ des femmes et moins de $10 \%$ des hommes ayant été victimes de tels faits, ont demandé de l'aide. 
* Déjà en 1976, aux Etats-Unis, DeFrancis (membre de l'American Human Society) attire l'attention sur le sujet, en publiant pour sa thèse de Doctorat, une étude menée sur les crimes sexuels commis sur des enfants. Il conclut: «les enfants victimes de violences sexuelles sont statistiquement plus nombreux que ceux qui subissent des violences physiques [...] tous les indices inclinent fortement à penser que son incidence, à ce niveau est infiniment plus important que ne pourrait le laisser supposer le nombre de cas rapportés. » [62]

S'en suivent plusieurs études nationales américaines sur les violences sexuelles sur mineurs, dont celles de Finkelhor et al. En 1990, ils montrent que $27 \%$ des femmes et $16 \%$ des hommes interrogés, déclarent avoir été victimes de violences sexuelles avant l'âge de 18 ans. L'âge moyen au moment des faits est de 9 ans et demi pour les femmes et le plus souvent l'agresseur est un membre de la famille ou une connaissance. Pour les hommes, l'âge moyen est de 9 ans et 9 mois, l'agresseur étant le plus souvent inconnu. [86]

En 1994, ils estiment le taux d'incidence pour les Etats-Unis en 1993, à 2,4 pour 1000 enfants. La prévalence de violences sexuelles avant 18 ans pour les pays d'Amérique du Nord est de $20 \%$ des femmes et $7 \%$ des hommes. [87]

En 1997, ils retrouvent un taux de prévalence de 1,9\% sur un an d'agressions sexuelles intrafamiliales sur des enfants de 0 à 17 ans. [89]

* Une enquête suédoise, de Edgardh et Ormstad (2000), réalisée chez 2153 étudiants de 7 ans, obtient les chiffres suivants :

- 3,1\% des garçons et $11,2 \%$ des filles avaient connu une situation d'agression sexuelle.

- L'âge moyen de survenue était de 9,1 ans pour les garçons et 9 ans pour les filles.

- 1,2\% des garçons et 7,1\% des filles signalaient des relations orales vaginales et/ou anales

- Les tentatives de suicides ou autres actes auto-agressifs étaient rapportés par 33,3\% des étudiants garçons et $30,4 \%$ des filles ayant été victimes ( $\mathrm{p}<0,001)$.

- Les troubles du sommeil et du comportement alimentaire, la consommation précoce d'alcool, l'expérimentation de drogues illicites ainsi que l'expérience sexuelle consentie avant 15 ans étaient plus souvent rapportés et de manière significative chez les filles ayant été victimes.

- Aucun garçon et très peu de filles victimes ne s'étaient confiés à un professeur, un professionnel de santé ou un travailleur social. [76] 
Une étude similaire a été réalisée par le CREA (Centre de Recherche sur l'Enfance et l'Adolescence) à Paris. Il retrouve au sein d'un échantillon de 1000 étudiants, que $8 \%$ des jeunes filles et $7 \%$ des jeunes hommes, signalent avoir connu une situation d'agression sexuelle. [37]

\subsection{LES ENQUETES EPIDEMIOLOGIQUES FRANÇAISES}

Le Service National d'Accueil Téléphonique pour l'Enfance Maltraitée (SNATEM), nous fournit des chiffres intéressants, bien que de sources uniquement déclaratives.

Avec l'Observatoire National de l'Enfance en Danger (ODAS), ils recensent les signalements de violences sexuelles. Après une régression de $13 \%$ entre 1995 et 1999, une croissance est rapportée entre 2000 et 2001 , passant de 5500 cas à 5900 cas. [167]

Ils nous donnent alors une idée de la répartition des types de violences sexuelles dont peuvent être victimes les mineurs (tableau 1).

Tableau 1: Répartition des mauvais traitements sexuels sur mineurs

\begin{tabular}{|lcccc|} 
& $\mathbf{1 9 9 8}$ & $\mathbf{1 9 9 9}$ & $\mathbf{2 0 0 0}$ & $\mathbf{2 0 0 1}$ \\
\hline $\begin{array}{l}\text { Climat } \\
\text { equivoque }\end{array}$ & $26 \%$ & $27 \%$ & $23,6 \%$ & $\mathbf{2 5 , 4 \%}$ \\
\hline Attouchement & $49 \%$ & $48 \%$ & $50,3 \%$ & $\mathbf{4 9 , 6 \%}$ \\
\hline Viol & $25 \%$ & $25 \%$ & $26,1 \%$ & $\mathbf{2 5 \%}$ \\
\hline Total & $100 \%$ & $100 \%$ & $100 \%$ & $100 \%$ \\
\hline
\end{tabular}

Source : SNATEM [189]

Avec le Collectif Féministe Contre le Viol (CFCV) en 1999, ils rapportent que les cas d'inceste représentent $20 \%$ des procès d'assises en France, $75 \%$ sont des situations d'agressions sexuelles sur enfants et $57 \%$ des viols sur mineurs. [38]

Ils obtiennent aussi des chiffres marquants concernant les viols sur mineurs (Tableau 2). Nous ne pouvons que constater une nette augmentation entre 1991 et 1998. De plus, en 1998, 481 cas sur 865 , soit un peu plus de $55 \%$ étaient des viols intrafamiliaux. 
Tableau 2: «Type » de viol et répartition par sexe

\begin{tabular}{|l|l|l|l|l|l|l|l|l|l|l|l|}
\cline { 2 - 11 } \multicolumn{1}{c|}{} & \multicolumn{19}{l|}{1991} & \multicolumn{1}{l|}{1995} & \multicolumn{1}{l|}{1996} & \multicolumn{1}{l|}{1997} & \multicolumn{2}{l|}{1998} \\
\cline { 2 - 12 } \multicolumn{1}{c|}{} & $\mathrm{F}$ & $\mathrm{M}$ & $\mathrm{F}$ & $\mathrm{M}$ & $\mathrm{F}$ & $\mathrm{M}$ & $\mathrm{F}$ & $\mathrm{M}$ & $\mathrm{F}$ & $\mathrm{M}$ \\
\hline $\begin{array}{l}\text { Viols sur } \\
\text { majeur }\end{array}$ & 327 & 26 & 511 & 43 & 415 & 25 & 393 & 45 & 511 & 47 \\
\hline Intrafamilial $^{*}$ & 44 & 0 & 53 & 0 & 92 & 0 & 106 & 3 & 85 & 1 \\
\hline Extra-familial & 283 & 26 & 458 & 43 & 323 & 25 & 287 & 42 & 426 & 46 \\
\hline $\begin{array}{l}\text { Viols sur } \\
\text { mineur }\end{array}$ & 599 & 36 & 1127 & 67 & 980 & 58 & 1019 & 90 & $\mathbf{7 8 3}$ & $\mathbf{8 2}$ \\
\hline Intrafamilial & 358 & 21 & 805 & 609 & 30 & 606 & 35 & $\mathbf{4 5 2}$ & $\mathbf{2 9}$ \\
\hline Extra-familial & 241 & 15 & 322 & 67 & 371 & 28 & 413 & 55 & $\mathbf{3 3 1}$ & $\mathbf{5 3}$ \\
\hline $\begin{array}{l}\text { Total des } \\
\text { viols }\end{array}$ & 926 & 62 & 1638 & 110 & 1395 & 83 & 1412 & 135 & 1294 & 129 \\
\hline
\end{tabular}

* Cette catégorie est exclusivement composée des "viols" par un compagnon (mari, concubin, etc....). Source : CFCV/Ciavaldini 2001 [43]

Jaspard et al. réalisent en 2001 une Enquête Nationale sur les Violences Envers les Femmes en France (ENVEFF). Parmi les 6970 femmes de 20 à 59 ans interrogées, 11,4\% avaient été victimes d'agressions sexuelles au cours de leur vie et la moitié des cas étaient avant 18 ans. Avant 18 ans, 6\% des femmes avaient été victimes d'agressions sexuelles (hors viol) et 3,7\% ont été victimes de viol. Selon eux, 9,7\% des femmes en France ont donc été victimes de violences sexuelles avant 18 ans. [129]

Selon Bouhet et al. (1992), 4,6\% des hommes ont été victimes de violences sexuelles avant l'âge de 18 ans. [23]

L’Observatoire National de la Délinquance et des Réponses Pénales (ONDRP) constate une progression de $11 \%$ des violences sexuelles sur mineurs, entre septembre 2011 et août 2012, par rapport à la même période sur l'année précédente. [1]

En 2010, une enquête menée par l'institut de sondage Ipsos, à la demande de l'Association Internationale des Victimes d'Inceste (AIVI), retrouve au sein d'un échantillon de 931 sujets, que 3\% déclarent avoir été victimes d'inceste et que 26\% connaissent au moins une personne victime d'inceste dans leur entourage. Ainsi, 2 millions de français seraient concernés. [126] 


\subsection{EPIDEMIOLOGIE DES FACTEURS DE RISQUE DE VIOLENCES SEXUELLES SUR MINEUR}

\subsubsection{Caractéristiques des agresseurs sexuels sur mineur}

Le pourcentage d'agresseurs masculins varie selon les études entre $85 \%$ et $100 \%$ (Finkelhor 1990 [86], Wolfe 2007 [227]). Lorsque la victime est une jeune fille, l'agresseur est un homme dans $92 \%$ des cas, une femme pour $2 \%$, à la fois un homme et une femme dans $4 \%$ des cas (sexe non spécifié dans 2\%). Concernant les garçons, l'agresseur est un homme dans $51 \%$ des cas, une femme 21\%, les deux 18\% (sexe non spécifié dans 10\%). (Dude et al 2005) [72].

Concernant l'âge, Holmes et Slap (1998) dans leur revue de publications, retrouvent que dans $40 \%$ des violences sexuelles sur mineurs, l'agresseur a moins de 20 ans et entre $13 \%$ et $18 \%$ moins de 13 ans. [123]

Grant et al. (2009) eux, ont identifié trois types de facteurs de risque pour un adolescent d'être auteur d'agressions sexuelles sur un enfant: avoir dans l'enfance été victime de violences physiques ou sexuelles, vivre dans une famille dysfonctionnelle et présenter des troubles psychiques (troubles des conduites, troubles de la personnalité, troubles thymiques). [112]

Entre $75 \%$ et $90 \%$ des enfants connaissent leur agresseur qui, pour la plupart, n'a pas de lien de parenté. (Badgley 1984, Finkelhor 1990) [9,86]. Cependant, l'étude d'une cohorte de 39 patients victimes de viols incestueux, consultant au Centre Médico Légal de Tours (DarvesBornoz J.M 1995), donne les chiffres suivants : pour 33\% des cas, l'auteur était le père, 28\% le beau père, $21 \%$ un oncle, $13 \%$ un frère, $3 \%$ un grand père et $3 \%$ un arrière grand-père. [51]

Ainsi, les facteurs parentaux associés aux violences sexuelles intrafamiliales sur mineurs, que l'on peut recenser sont : l'âge inférieur à 18 ans avec un faible niveau d'étude de la mère, une grossesse non désirée, un faible sentiment de compétence parentale, une consommation de drogue ou d'alcool, un problème de santé mentale chez les parents ou encore des difficultés conjugales (Stier et al. 1993 [202], Black et al. 2001 [18], Finkelhor et Baron 1986 [85], Fleming et al 1997 [91], Wolfe 2007). 
Dans ce sens, en France, le SNATEM (1999) retrouve pour des situations d'agressions intrafamiliales, des difficultés relationnelles familiales à $73 \%$, des contextes de séparation ou de divorce des parents à 43\%, l'alcoolodépendance à $11 \%$, ainsi que la précarité à $7 \%$. [194]

\subsubsection{Facteurs de vulnérabilité des victimes}

Les victimes mineures d'agressions sexuelles sont $75 \%$ à $85 \%$ de sexe féminin, ainsi elles ont trois fois plus de risque d'être victimes que les garçons (Black et al. 2001, Wolfe 2007). Les âges critiques sont entre 6 et 7 ans ainsi qu'entre 10 et 12 ans (Wolfe 2007). Elles semblent aussi plus vulnérables aux agressions sexuelles intrafamiliales (Finkelhor et al. 1990, Tourigny 1991 [207], Wolfe 2007). Ce risque est encore majoré lorsque l'environnement comprend un beau père, un parent adoptif ou un parent d'accueil (Finkelhor et Baron 1986, Wolfe 2007).

Quelque soit le sexe du sujet mineur, pour $45 \%$ des victimes d'attouchements sexuels la première agression s'est déroulée entre 6 et 11 ans, alors que $69 \%$ des viols le sont à l'adolescence. (Tourigny et al. 2008) [208]

D'autres caractéristiques personnelles et environnementales peuvent définir une vulnérabilité. Notre attention doit donc être toute particulière face à des jeunes présentant un déficit intellectuel, un handicap physique, des difficultés scolaires, un isolement social, mais aussi certains manifestants un besoin accru d'attention et surtout les jeunes ayant par le passé déjà été victimes de violences physiques. (Finkelhor et Baron 1986, Fleming et al. 1997, Black et al. 2001, Wolfe 2007). 


\section{DEUXIEME PARTIE}

\section{LES TROUBLES POST-TRAUMATIQUES CHEZ L'ADULTE : CLINIQUE ET EVALUATION DIAGNOSTIQUE}




\section{LES TROUBLES CHEZ L'ADULTE AYANT ETE VICTIME DE VIOLENCES SEXUELLES DANS L'ENFANCE}

A la suite de violences sexuelles, jusqu'à $40 \%$ des enfants peuvent être initialement asymptomatiques ou ne présenter que peu de symptômes (Finkelhor et Beliner, 1995) [88]. Ce sont ces sujets, qui sont les plus susceptibles de présenter une dégradation globale avec le temps (Gomes-Schwartz, 1990) [111]. Les victimes ne sont donc jamais indemnes des maltraitances vécues dans l'enfance. Les conséquences, variables avec l'âge, peuvent aussi en fonction des individus, avoir diverses présentations et gravités.

Frank et Putman en 2003, dans leur revue de littérature, retrouvent chez l'adulte, associées aux violences sexuelles durant l'enfance, des pathologies codifiées dans le DSM-IV, à savoir : épisodes dépressifs majeurs, troubles de la personnalité type borderline, somatisations, abus de substances, états de stress post-traumatique, troubles dissociatifs de la personnalité et troubles du comportement alimentaire, mais aussi des troubles du comportement et des altérations neurobiologiques. [99]

Encore plus récemment, une étude longitudinale néo-zélandaise de Fergusson et al. (2013) a permis d'analyser les liens entre les maltraitances sexuelles dans l'enfance et le développement de diverses séquelles à l'âge adulte. Ils ont suivi une cohorte née en 1977, de 1265 sujets, de la naissance, puis à 4 mois, 1 an, annuellement jusqu'à 16 ans et enfin à 18 , 21, 25 et 30 ans. En calculant les valeurs de covariances ajustées sur les facteurs de confusion, d'erreur standard et de niveau de significativité des associations, ils montrent des associations entre la notion d'agression sexuelle avant l'âge de 16 ans et l'augmentation du risque de dépression majeure $(\mathrm{Cov}=0,426 ; \mathrm{ES}=0,094 ; \mathrm{p}<0,001)$, de troubles anxieux $(0,364 ; 0,089$; $<0,001)$, d'idées suicidaires $(0,395 ; 0,089 ;<0,001)$, de tentative de suicide $(1,863 ; 0,403$; $<0,001)$, de dépendance à l'alcool $(0,374 ; 0,118 ;<0,002)$ et de dépendance à d'autres drogues $(0,425 ; 0,113 ;<0,001)$. A l'âge de trente ans, l'association est marquée avec les symptômes d'ESPT $(0,120 ; 0,051 ; 0,017)$, de diminution de l'estime de soi $(-0,371,0,181$, $0,041)$ et de diminution de satisfaction de vie globale $(-0,510 ; 0,189 ; 0,007)$. Ils ont de plus obtenu un lien avec l'âge plus précoce de début d'activité sexuelle $(-0,318 ; 0,091 ;<0,001)$, d'augmentation du nombre de partenaires $(0,175 ; 0,035 ;<0,001)$, d'augmentation des recours aux services médicaux pour des problèmes de santé physique $(0,105 ; 0,023 ;<0,001)$ ainsi qu'une augmentation du recours aux aides sociales $(0,310 ; 0,099 ;<0,002)$. [83] 
En utilisant les données de la littérature, nous exposerons les conséquences psychiques et organiques se manifestant à l'âge adulte, des violences sexuelles dans l'enfance.

\subsection{PSYCHOPATHOLOGIE}

Les publications soulignent le lien entre certains troubles à l'âge adulte, dont des pathologies psychiatriques, et un antécédent de violences sexuelles.

L'impact psychique d'un traumatisme dans l'enfance, persiste la plupart du temps à l'âge adulte, se manifestant par des épisodes dépressifs, des troubles anxieux, une dépendance à l'alcool et aux drogues, des troubles de personnalités ainsi que des troubles du comportement alimentaire (Rorty et al. 1996, Wonderlich et al., 2001). [184,229]

La recherche menée par Nelson et al. (2002) chez 1991 paires de jumeaux, montre que chez le jumeau ayant un antécédent de violences sexuelles dans l'enfance, les taux de dépression, de tentative de suicide, de dépendance à l'alcool et la nicotine, de trouble des conduites, d'anxiété sociale, de viol après 18 ans et de divorce sont supérieurs. [159]

La psychopathologie décrite chez des sujets soumis à des traumatismes différents, présente bien évidemment certains caractères spécifiques, mais reste globalement assez similaire. L'état de stress post-traumatique regroupe certaines des manifestations fréquentes dans les suites de guerre, d'accident ou d'agression sexuelle.

\subsubsection{L'Etat de Stress Post-Traumatique (ESPT) et autres troubles névrotiques}

Les chiffres portant sur l'ESPT, tous types de traumas confondus, dont nous disposons, sont relativement hétérogènes, de par les zones géographiques explorées et les méthodes d'investigations différentes utilisées.

L'étude française, «Santé Mentale en Population Générale » (SMPG, Vaiva et al.) de 2008, donne une prévalence pour l'ESPT de 0,7\% [215]. L'étude ESEMeD (European Study of the Epidemiologigy of Mental Disorder Survey, Alonso et al. 2004), estime une prévalence vie 
entière de l'ESPT chronique à 1,9\% [4]. Un chiffre bien plus important, est révélé par l'enquête américaine de Kessler en 2005, avec une prévalence au cours de la vie à 6,8\%. [137]

La revue de littérature de De Venter (2013), montre à partir de 65 publications, que les plus importants facteurs de risque associés aux troubles anxieux sont un vécu de violences intrafamiliales et de violences sexuelles dans l'enfance [64]. Chen (2010) dans sa métaanalyse, obtient une association statistiquement significative entre l'apparition d'un trouble anxieux et le fait d'avoir un antécédent d'agressions sexuelles dans l'enfance. [41]

On constate alors, que parmi ces publications abordant les maltraitances dans l'enfance, l'état de stress post-traumatique est la pathologie souvent citée. Steil et Straube (2002) décrivent un Etat de Stress Post-Traumatique chez 80\% des enfants victimes de violences sexuelles. [196]

Plusieurs enquêtes soulignent alors les troubles comorbides à l'ESPT. L'étude SMPG (Vaiva, 2003) nous donne, associés à l'ESPT, les chiffres suivants : un épisode dépressif majeur dans $39,1 \%$ des cas, une dépression récurrente à 17,5\%, un trouble anxieux généralisé à $61,5 \%$, un trouble panique à $18,6 \%$, une phobie sociale à $15,9 \%$, des troubles liés à l'alcool à $13,4 \%$ et aux drogues à 11,4\%, ainsi qu'un risque suicidaire 30,9\%. [216]

Ainsi, peuvent se manifester tout un panel de pathologies, la plus représentée étant l'épisode dépressif majeur (EDM). Cependant, la nature de l'association ESPT/EDM n'est pas clairement définie. Dans le cadre d'un évènement traumatique, présentent-elles les mêmes caractères de vulnérabilité, ou sont-elles deux manifestations indépendantes ?

\subsubsection{Troubles thymiques dont l'épisode dépressif majeur}

Les études abordant différents évènements traumatiques estiment la prévalence des manifestations dépressives chez des patients présentant un ESPT entre 30 et 50\%. (Nixon 2004 [162], Blanchard 1998 [20]). L'étude «Adverse Chilhood Experiences » (ACE, Chapman 2004) montre une relation graduelle entre le nombre d'évènements potentiellement délétères dans l'enfance et la fréquence des troubles dépressifs récents et vie entière $(\mathrm{p}<0,0001) .[39]$ 
Une association statistiquement significative est retrouvée entre un antécédent de violences sexuelles dans l'enfance et des troubles thymiques, avec en première ligne, la symptomatologie dépressive chez l'adulte (Paolucci et al. 2001 [171], Polusny et Follette 1995 [178], Neumann et al. 1996 [160]). Le suivi de 1000 nouveau-nés en Nouvelle-Zélande (Fergusson et al. 1996 a,b) a permis d'estimer, pour la dépression majeure, une augmentation de l'odds ratio de 8,1 entre les sujets avec des notions de violences sexuelles dans l'enfance et ceux indemnes, et de 11,8 pour les tentatives de suicides. [81,82]

$\mathrm{Au}$ sein de cette population, la présentation clinique de la dépression aura quelques particularités. Le premier épisode est souvent plus précoce (Gisese 1998 [106]), les épisodes plus longs (Zlotnick et al 2001 [239]), les symptômes neurovégétatifs peuvent être inversés (Levitan et al. 1998 [149]), avec une hypersomnie, une augmentation de l'appétit et une prise de poids. D'autres facteurs peuvent influencer la sévérité des symptômes: le lien avec l'agresseur, la durée des violences, le recours à la violence physique (Trickett et al. 2001 [209]) et le sexe masculin (Gold et al. 1999 [107]).

\subsubsection{Les troubles de la personnalité}

Parmi des patients présentant un ESPT, on peut identifier pour certains, un trouble de la personnalité, notamment limite, paranoïde ou antisocial. (Golier et al 2003 [110])

Le type état limite, largement majoritaire, présente des similitudes avec l'ESPT. L'irritabilité, les troubles dissociatifs et du comportement, dont les passages à l'acte, ne doivent pas entraver leur distinction.

Les sujets victimes de maltraitances dans l'enfance présentent fréquemment un trouble de la personnalité. C'est le constat rapporté par l'étude de 105 adultes entre 18 et 64 ans de Tyrka et al. (2009) [212]. Chez les adultes ayant été victimes d'inceste durant l'enfance, un trouble de personnalité est souvent en lien avec un attachement «insécure », alors que la dépression et l'ESPT le sont avec la sévérité des violences (Alexander et al. 1998 [2]). On comprend en effet aisément, que lorsque l'agresseur est une des figures d'attachement, la personnalité se structure par la suite vers l'état limite. En abordant différemment cette association, on retrouve, au sein de population de patients limites, une notion d'agressions sexuelles dans l'enfance, chez environ 75\% d'entre eux (Linehan, 1993 [151]). 
Certains voyaient dans les traumatismes sexuels de l'enfance, une étiologie au trouble de personnalité type limite, reviennent actuellement sur leur affirmation (Zanarini et al 2000 [235]). Cependant, les chiffres les associant interpellent, les modalités de leur lien restent donc à éclaircir.

\subsubsection{Les addictions}

L'ESPT est après la dépression le trouble de plus fréquemment associé aux addictions selon Kessler (1995) [135].

Le Docteur Sigward en 2006, montre dans son étude réalisée à l'hôpital Tenon que 8\% des patients toxicomanes du groupe étudié ont été témoins ou victimes de scènes potentiellement traumatisantes et que parmi eux, 53\% ont subi au moins une fois des attouchements sexuels ou un viol. [192]

Concernant l'alcool, Dude (2006) évalue que 93,6\% des sujets adultes ayant été victimes d'agressions sexuelles dans l'enfance ont déjà consommé de l'alcool, contre $88 \%$ chez les sujets indemnes de ce type d'évènement $(\mathrm{OR}=2,0 ; \mathrm{IC} 95 \%=[1,6-2,5])$. L'initiation précoce est aussi constatée avec une consommation avant l'âge de 14 ans chez 13,4\% des sujets versus $7 \%(\mathrm{OR}=2,8 ; \mathrm{IC} 95 \%=[2,3-3,5]) .[73]$

L'étude «Adverse Childhood Experiences » (ACE, Anda et Felliti 1999 [5]) réalisée chez 9215 individus, montre une forte association entre le tabagisme et les évènements traumatiques dans l'enfance. En comparant des sujets indemnes versus des sujets avec un vécu traumatique, ils obtiennent une augmentation du risque d'initiation au tabac avant 14 ans $(\mathrm{OR}=5,4$; IC95\%=[4,1-7,1]), d'une consommation occasionnelle $(\mathrm{OR}=2,8$; IC95\%=[ 2,6$3,8])$, de consommation régulière $(\mathrm{OR}=2,1 ; \mathrm{IC} 95 \%=[1,6-2,7])$ et d'être un gros consommateur $(\mathrm{OR}=2,8$; IC95\%=[1,9-4,2]). Le tabagisme actif, se trouve donc lui aussi majoré, chez les victimes d'agressions sexuelles dans l'enfance (27,4\% VS 17,7\% ; $\mathrm{OR}=1,51 ; \mathrm{IC} 95 \%=[1,35-1,69])($ Ford, 2011) [95]. 
L'usage de toxiques pour ces patients peut s'apparenter à de l'automédication face aux symptômes handicapants que sont les troubles du sommeil, l'anxiété et les symptômes intrusifs, avec une mise à distance des affects. L'intrication forte avec les troubles précédemment évoqués font que les addictions, répondent de manière satisfaisante aux prises en charge de type cognitivo-comportementale (Tison 2002, [206]) que nous exposerons par la suite.

\subsubsection{Comportement suicidaire}

L'étude ACE (Dude 2001) donne en population générale, une prévalence de tentative de suicide au cours de la vie de 3,8\%. Ce risque augmente de 2 à 5 fois quelque soit le type d'évènement délétère dans l'enfance. Les sujets présentant plusieurs expériences négatives ont alors une prévalence de 35,5\% (OR ajusté 31,1\% ; IC95\%=[20,6-47,1]). [71]

Avec pour les hommes un OR=3,7 (IC95\%=[2,24-4,67]) et pour les femmes un OR=3,3 $($ IC95\% $=[1,4-7,9])$, Brezo (2008) retrouve un risque de tentative de suicide majoré chez des sujets agressés sexuellement dans l'enfance. En soulignant le lien entre agression sexuelle dans l'enfance et comportement suicidaire, il montre de plus une majoration de ce risque lorsqu'il s'agit de victimes d'inceste [30]. Ainsi, pour l'enquête AIVI, parmi les 341 victimes d'incestes interrogées, 53\% ont fait une tentative de suicide. [7]

Selon l'enquête SMPG de Vaiva et al. (2008), l'ESPT induit quatre fois plus de tentatives de suicide et sept fois plus de suicides [215]. Le risque suicidaire, bien que déjà élevé chez des sujets ayant un ESPT isolé (Oquendo et al. 2005), peut aussi être majoré par les addictions, les troubles thymiques et de la personnalité que l'on sait fréquemment comorbides à l'ESPT. [169]

Les comportements suicidaires, de toute évidence marqués au sein de cette population de patients, se doivent d'être recherchés. L'évaluation par le clinicien doit tenir compte des caractéristiques associées, influençant le risque suicidaire, afin d'en améliorer la prévention. 


\subsubsection{Les troubles du comportement alimentaire}

Pour Wonderlich (1997) et Neumark-Sztainer (2000), un traumatisme dans l'enfance notamment sexuel, est un facteur de risque prédisposant aux troubles du comportement alimentaire (TCA) à l'adolescence ainsi qu'à l'âge adulte [228,161]. Selon Rodrigez (2005), $45 \%$ des patients avec des TCA ont une histoire de maltraitance entre autre d'ordre sexuel. [182]

Sullivan (1995) centre son étude sur le lien entre boulimie et agressions sexuelles dans l'enfance et évalue la survenue de comorbidités. Ainsi, il retrouve chez 87 patientes, prises en charge pour boulimie, un antécédent de violence sexuelle dans l'enfance. 29\% d'entre elles présentent un trouble bipolaire type II, alors que chez les patientes sans cet antécédent, seulement $6 \%$ de trouble bipolaire étaient observés $(\mathrm{p}=0,004), 63 \%$ une addiction versus $37 \%$ $(\mathrm{p}=0,01)$ et $46 \%$ une tentative de suicide versus $24 \%(\mathrm{p}=0,04)$. Selon lui, ce type de traumatisme rend plus précoce la survenue d'une boulimie mais n'a pas d'influence sur sa sévérité. [203]

Kong (2009), après avoir constaté chez 73 patients que les négligences psychologiques physiques et sexuelles, pouvaient être prédictives de pathologies du comportement alimentaire, conclut à la nécessité de rechercher un traumatisme dans l'enfance chez des patients suivi pour des TCA. Il note de plus, une forte association entre TCA, traumatisme dans l'enfance et dépression. [141]

Cherchant à expliquer la fonction des troubles du comportement alimentaire chez ses patients, trois hypothèses psychopathologiques peuvent être avancées. La première vient de la nécessité de contrôle, ici du poids, alors que précisément l'évènement traumatique a marqué un épisode de perte de contrôle. La seconde est la modification corporelle induite par un surpoids ou une maigreur, gommant les caractères sexuels secondaires. La troisième est l'état de dissociation recherché par ses sujets et provoqué au moment des crises. 


\subsubsection{Troubles psychotiques}

Whitfield (2005) observe un lien statistiquement significatif entre des notions de traumatisme dans l'enfance et d'hallucinations, indépendamment de consommations de toxiques. Ainsi, $3,1 \%$ des sujets ayant vécu une agression sexuelle dans l'enfance rapportent des manifestations hallucinatoires, contre $1,7 \%$ au sein de sujets non victimes. [225]

Cependant, pour Bendall (2011), il semble que l'on ne puisse pas établir de lien de causalité clair entre un trouble psychotique et un vécu traumatique dans l'enfance. [14]

De plus, selon la méta-analyse de Sideli (2012), aucune étude n'a apporté de résultat probant, soutenant l'hypothèse que des maltraitances dans l'enfance soient une condition nécessaire et suffisante pour développer un trouble psychotique. [191]

Alors que le rôle étiologique est sujet à controverse, certains chercheurs se tournent vers l'hypothèse gène-environnement (Van Winkel, 2013). Ils abordent en synergie, psychologie et neurobiologie chez des patients psychotiques, ayant des notions de traumatisme dans l'enfance. [219]

\subsubsection{Revictimisation}

La revictimisation peut être définie comme la répétition au cours de la vie d'expériences traumatiques. Certains se limitent aux revictimisations d'ordre sexuel, d'autres incluent plusieurs types d'évènements traumatiques, les définitions de ce terme varient d'une étude à l'autre.

Coid (2001) a voulu étudier la relation entre un traumatisme dans l'enfance et la revictimisation une fois adulte. Chez 1207 les femmes inclues, un rapport sexuel non désiré avant 16 ans est retrouvé associé à des climats de violences physiques des parents ou tuteurs $(\mathrm{OR}=3,58 ; \mathrm{IC} 95 \%=[2,06-6,20])$ et de viol $(\mathrm{OR}=2,70 ; \mathrm{IC} 95 \%=[1,27-5,74]) ;$ mais aussi à l'âge adulte, de violences domestiques (OR=3,54; IC95\%= [1,52-8,25]), de viol $(\mathrm{OR}=2,84$; IC95\%=[1,09-7,35], ou encore d'autres types de traumas (OR=3,85; IC95\%=[2,23-6,63]). [45] 
Pour Banyard et al (2001), les femmes ayant été victimes de violences sexuelles dans l'enfance sont plus fréquemment sujettes à d'autres évènements traumatiques au cours de leur vie. [11]

Walsh (2012) a réalisé aux Etats-Unis, une étude évaluant la prévalence de victimes d'agressions sexuelles, de revictimisation sexuelle et de manifestation d'ESPT, au sein de populations de femmes à trois tranches d'âges différentes. Ainsi, parmi les 11,7\% d'adolescentes entre 12 et 17 ans ayant été victimes d'agressions sexuelles, 52,7\% ont un vécu de revictimisation, dont $20 \%$ présentent un ESPT. Au sein des 12,5\% des étudiantes victimes, 50\% signalent une revictimisation, dont 40\% manifestent un ESPT. Pour les femmes adultes, on retrouve $20 \%$ de victimes, avec $58 \%$ de revictimisation d'ordre sexuel dont $27,2 \%$ avec un ESPT. [221]

Présenter à la fois un ESPT et un trouble de la personnalité majore le risque de mise en danger et donc de connaître d'autres évènements traumatiques (Debray 2006 [61]). On peut alors penser que des facteurs tels que la nature du soutien familial et social, le type de traumatisme, l'usage de toxiques ou la dépression, contribuent au lien entre les violences sexuelles dans l'enfance et la revictimisation.

\subsubsection{Troubles de l'attitude sexuelle et de la sexualité}

Les premières étapes du développement psychosexuel se font dans l'enfance et à l'adolescence, les agressions sexuelles se déroulant lors de ces phases clefs, vont de toute évidence avoir des conséquences sur la sexualité de ces individus.

Ainsi, Hotte (1992) observe chez des jeunes filles entre 8 et 14 ans, victimes d'inceste, des comportements plus sexualisés. Elles sont moins pudiques, abordent avec aisance des sujets masculins plus âgés et ont avec les adultes des interactions sexualisées. [125]

Windom et Ames (1994) mettent en évidence une part significativement plus importante d'arrestations pour crime sexuel ou prostitution, chez les sujets ayant un vécu de violences sexuelles dans l'enfance et ce quelque soit leur sexe. Majoration non observée chez les individus avec des notions de simples violences physiques ou de négligence. [226] 
Au sein de ce type de population, un risque accru de grossesse précoce est constaté (Fiscella 1998 [90], Rainey 1995 [180]) avec plus de complications de la parturition ainsi que de bébés hypotrophes. Stevens-Sinon et Reichert (1994) relient ces observations au stress, la dépression, l'isolement social et la toxicomanie. [201]

Stephenson (2012) rapporte une plus grande fréquence de troubles du désir, de l'excitation, de la lubrification et de l'orgasme chez les femmes avec des antécédents d'agressions sexuelles dans l'enfance, engendrant un sentiment de détresse sexuelle. Ces troubles de la fonction sexuelle sont encore majorés lorsque l'agression était intrafamiliale. [200]

\subsubsection{Les séquelles neurobiologiques comme modèle explicatif de la psychopathologie}

Des altérations neuro-anatomiques et physiologiques ont été mises en évidence chez les victimes de violences sexuelles dans l'enfance.

Ainsi, un volume cérébral inférieur à celui de la population générale est constaté chez des enfants victimes de maltraitances. Une diminution marquée du volume des zones médiane et postérieure du corps calleux, associée à des symptômes d'ESPT et de dissociation est rapportée, et ce majoritairement dans la population masculine (De Bellis, 1999 [59]). Une diminution du volume hippocampique a été mesurée par des études utilisant l'imagerie par résonance magnétique (Driessen 2000, Bremmer 1997). [67,27]

Putnam et Trickett (1997) ont pu observer au sein d'un échantillon de femmes avec un vécu de violences sexuelles, une altération de l'axe hypothalamo-hypophyso-surrénalien, du système immunitaire ainsi qu'un dysfonctionnement du système nerveux sympathique. [179]

Certains mettent en relation ces altérations neurophysiologiques et la psychopathologie décrite précédemment. Par exemple, les manifestations d'agressivité des personnalités borderlines qui présentent une altération structurelle du cortex préfrontal ventro-latérale. (Norandotti, 2013). [163]

Les séquelles neurobiologiques ne sont pas les seuls troubles organiques constatés. 


\subsection{LES PATHOLOGIES ORGANIQUES}

Les plaintes somatiques sont souvent pour ces patients, le motif d'entrée dans les soins. Les pathologies telles que les maladies sexuellement transmissibles sont communément reconnues comme devant interpeller les soignants. Cependant, d'étonnantes associations sont retrouvées par les publications entre un antécédent de violence sexuelle et certaines manifestations organiques moins évidentes.

- Maladies sexuellement transmissibles :

La population de patients que nous étudions, présentent comme nous avons pu le voir précédemment, des troubles de l'attitude sexuelle et des mises en danger à répétition. Il est alors aisé de faire le lien avec la majoration du risque de maladies sexuellement transmissibles existant dans cette même population.

La méta-analyse de Norman (2012), retrouve une majoration du risque de maladies sexuellement transmissibles et de comportements sexuels à risque $(\mathrm{OR}=1,78$; IC95\%=[ 1,502,10]) au sein de sujets adultes, ayant vécu des maltraitances physiques dans l'enfance. [164]

Les publications associant le risque plus précisément lié au VIH (Virus d'Immunodéficience Humaine) et les maltraitances sexuelles dans l'enfance sont assez riches. (Wyatt 2002 ; Allers 1993 ; Zeiler 1991) [231,3,237]

Il est aussi intéressant d'aborder le risque de maladies sexuellement transmissibles, directement lié aux maltraitances et pas seulement celui associé aux troubles secondaires. L'observation doit donc être faite au sein d'une population de mineurs. Ainsi, les centres de santé d'Atlanta aux Etats-Unis, participant au programme de surveillance nationale du VIH (Virus d'Immunodéficience Humaine) et SIDA (Syndrome d'Immunodéficience Acquise), ont recensé en décembre 1996, 9136 enfants HIV positifs, au sein desquels 24 cas avaient été examinés pour des antécédents de maltraitances sexuelles. (Lindegren 1998). [150] 
- Maladies auto-immunes et Diabète

Boscarino en 2004, a menée une étude sur 2490 vétérans du Viêtnam, montrant ainsi un lien entre l'ESPT chronique et la prévalence de maladies auto-immunes incluant la polyarthrite rhumatoïde, le psoriasis, le diabète insulinodépendant et les dysthyroïdies. Ces sujets présentent des taux plus élevés de lymphocytes $\mathrm{T}$, une réponse immune hypersensible au test cutané retardé, des taux d'immunoglobulines M plus élevés avec des taux inférieurs de déhydroépiandrostérone. Les anomalies de ces marqueurs biologiques sont alors compatibles avec des troubles inflammatoires, dont des maladies cardiovasculaires et auto-immunes. [22]

\section{- Le poids}

Des publications ont analysé l'Indice de Masse Corporel (IMC) chez des patients avec un vécu de traumatisme sexuel dans l'enfance. Roenholt et Beck (2012) ont réalisé au Danemark, une étude de probabilité randomisée et stratifiée entre 2008 et 2009 au sein de 2981 sujets nés en 1984. Ils recherchaient un lien entre la symptomatologie d'ESPT les maltraitances dans l'enfance et le poids. Selon eux, la symptomatologie d'ESPT et les maltraitances dans l'enfance sont associées de manière significative avec le faible IMC ainsi qu'avec le surpoids et l'obésité. En analysant les résultats par catégorie de maltraitance, ils constatent que les sujets victimes de violences sont $13,6 \%$ à être en surpoids et $11,9 \%$ sont obèses. Contrairement au poids insuffisant qui lui est plus fréquemment associé aux maltraitances de type émotionnel. [183]

- Céphalées et autres plaintes algiques

L'étude ACE de 2010 réalisée à partir de 17337 adultes montre une relation graduelle entre la prévalence de céphalées fréquentes et le nombre d'évènements dans l'enfance. Le risque de céphalées fréquentes augmente de plus de deux fois chez les sujets ayant de multiples expériences traumatiques par rapport aux sujets indemnes $(\mathrm{OR}=2,1 ; \mathrm{IC} 95 \%=[1,8-2,4])$. [6]

Paras (2009) a regroupé dans sa méta-analyse les résultats de 23 études publiées entre 1980 et 2008, réalisant alors une évaluation de 460 sujets. Il obtient des associations significatives entre un vécu d'agression sexuelle, et ce quelque soit l'âge de la victime au moment des faits, avec de multiples manifestations douloureuses, dont les troubles fonctionnels gastrointestinaux $(\mathrm{OR}=2,43$; IC95\%=[1,36-4,31]), les douleurs chroniques non spécifiques 
$(\mathrm{OR}=2,20 ; \quad \mathrm{IC} 95 \%=[1,54-3,15])$ et les douleurs pelviennes chroniques $(\mathrm{OR}=3,35$; IC95\%=[1,73-4,30] ). Lorsqu'il s'agit de viol, l'association significative est retrouvée avec la fibromyalgie $(\mathrm{OR}=3,35 ; \mathrm{IC} 95 \%=[1,51-7,46])$. [172]

- Chez les sujets âgés

Les travaux de Stein et Barrett-Connor (2000) retrouvent au sein d'une population de 1359 sujets dont la moyenne d'âge est de 75 ans, des déclarations d'agressions sexuelles chez 5,4\% des hommes et $12,7 \%$ des femmes. Parmi la population féminine, le vécu d'agression sexuelle est retrouvé associé à une majoration du risque d'arthrite $(\mathrm{OR}=1,76$; $\mathrm{IC} 95 \%=[1,13-2,76])$ et de cancer du sein $(\mathrm{OR}=2,21$; IC95\%=[1,12-4,33] 95\%). Le fait que les agressions soient répétées dans le temps multiplie le risque entre 2 à 3 par rapport à un évènement isolé. Pour les hommes, la seule association statistiquement significative était obtenue avec les pathologies de la thyroïde (OR=4,62; IC95\%=[1,08-20,3]). [197] 


\subsection{THE ADVERSE CHILDHOOD EXPERIENCES STUDY}

Déjà citée à de nombreuses reprises, cette étude menée chez plus de 17000 sujets aux EtatsUnis est majeure. Elle a fait l'objet de plus d'une cinquantaine d'articles et au moins autant d'autres l'ont utilisée comme base pour des recherches plus avancées dans le monde entier. Elle a évalué les conséquences d'évènements négatifs dans l'enfance et ce dans tous les domaines, aussi bien médical que familial, social et économique. Ces «expériences négatives » regroupent les maltraitances physiques, émotionnelles et sexuelles, mais aussi les dysfonctionnements au sein du foyer, tels que avoir été témoin de violences domestiques, grandir avec un criminel dans le foyer ou encore avec une personne ayant une maladie mentale ou un abus de substance.

La publication de Felliti (1998) nous permet de faire une synthèse des observations précédentes. Réalisée chez 9508 sujets, elle conclut à une relation graduelle entre l'ampleur de l'exposition à des expériences négatives pendant l'enfance, sans distinction particulière pour les agressions sexuelles, et l'augmentation du risque de comportements et pathologies chez l'adulte, certaines pouvant entrainer une surmortalité. [78]

L'étude ACE a réussi à résumer la chronologie des conséquences des expériences négatives dans l'enfance par le schéma suivant :

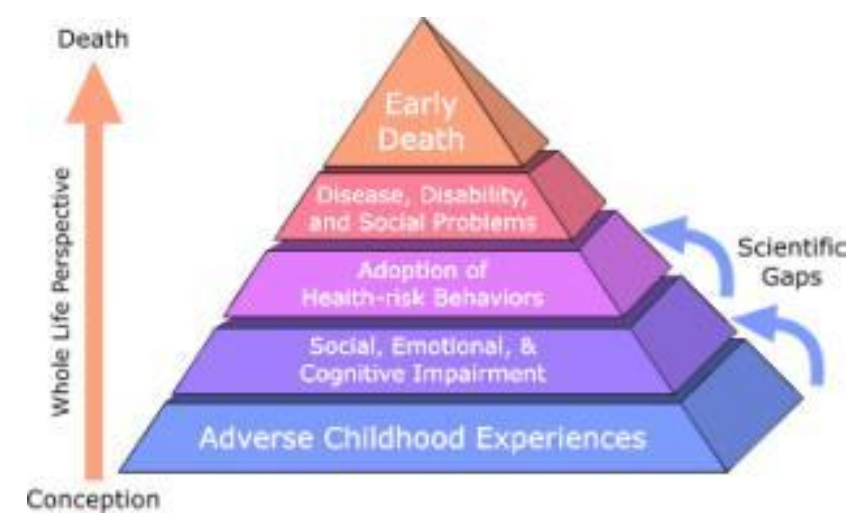

Source : http://acestudy.org/ 
Ci-dessous le tableau 3, résumant les principaux troubles dont on observe une majoration du risque chez les adultes ayant été exposés dans leur enfance à quatre évènements de vie néfastes. Les odds ratio sont tous statistiquement significatifs.

Tableau 3: Conséquences de l'exposition à quatre évènements de vie pendant l'enfance

\begin{tabular}{|l|c|l|l|}
\hline Conséquences & Facteur de risque & Conséquences & Facteur de risque \\
\hline Tabagisme & $\times 2,2$ & Obésité sévère & $\times 1,6$ \\
\hline Dépression durant plus de 2 semaines & $\times 4,6$ & Absence d'activité physique et de loisir & $\times 1,3$ \\
\hline Tentatives de suicide & $\times 12,2$ & Toxicomanie & $\times 4,7$ \\
\hline Alcoolisme & $\times 7,4$ & Toxicomanie Parentérale & $\times 10.3$ \\
\hline Maladie sexuellement transmissible & $\times 2,5$ & Coronaropathie & $\times 2,2$ \\
\hline Cancer & $\times 1,9$ & Agressions & $\times 2,4$ \\
\hline Broncho-pneumopathie chronique obstructive & $\times 3,9$ & Diabète & $\times 1,6$ \\
\hline Hépatite & $\times 2,5$ & Fractures & $>50$ partenaires sexuels \\
\hline Etat de santé précaire & $\times 2,2$ & $\times 1,6$ \\
\hline
\end{tabular}

Source : ACE Felliti 1998

Une limite peut toutefois être opposée à tous ces résultats. Tant le phénomène des agressions sexuelles est fréquent qu'il est aisé de trouver un lien avec des manifestations psychiques ou physiques, le lien obtenu n'étant alors pas systématiquement statistiquement significatif.

Il semble aussi caricatural de faire le raisonnement inverse en cherchant à associer de manière trop simple un trouble de personnalité type limite, les troubles de la sexualité ou encore les addictions et les comportements suicidaires à une notion de violence sexuelle. 


\section{CRITERES DES MANUELS DIAGNOSTIQUES}

\subsection{L'ETAT DE STRESS POST-TRAUMATIQUE (ESPT) DANS LE DSM-IV}

Le DSM-IV-TR [68] ou Manuel diagnostique et statistique des troubles mentauxquatrième version révisée, établi par la Société Américaine de Psychiatrie en 2000, caractérise l'état de stress post-traumatique (ESPT) à partir des six critères suivant, de A à F :

A. La confrontation à l'évènement traumatique

Le sujet a été exposé à un évènement traumatique dans lequel les deux éléments suivants étaient présents :

- 1. Le sujet a vécu, a été témoin ou a été confronté à un évènement ou à des évènements durant lesquels des individus ont pu trouver la mort ou être très gravement blessés ou bien ont été menacés de mort ou de blessures graves ou bien durant lesquels son intégrité physique ou celle d'autrui a pu être menacée.

2. La réaction du sujet à l'évènement s'est traduite par une peur intense, un sentiment d'impuissance ou d'horreur.

B. Symptômes d'intrusion

L'évènement traumatique est constamment revécu de l'une (au moins) des façons suivantes :

1. Souvenirs répétitifs et envahissants de l'évènement provoquant un sentiment de détresse et comprenant des images, des pensées ou des perceptions.

- 2. Rêves répétitifs de l'évènement provoquant un sentiment de détresse.

口 3. Impression ou agissements soudains «comme si » l'évènement traumatique allait se reproduire (illusions, hallucinations, flash-back).

4 4. Sentiment intense de détresse psychologique lors de l'exposition à des indices externes ou internes évoquant ou ressemblant à un aspect de l'évènement traumatique en cause.

$\square$ 5. Réactivité physiologique lors de l'exposition à des indices internes ou externes pouvant évoquer un aspect de l'évènement traumatique en cause. 
C. Symptômes d'évitement et d'émoussement

Evitement persistant des stimuli associés au traumatisme et émoussement de la réactivité générale (ne préexistant pas au traumatisme), avec au moins trois des manifestations suivantes :

$\square$ 1. Efforts pour éviter les pensées, les sentiments ou les conversations associées au traumatisme.

口 2. Efforts pour éviter les activités, les endroits ou les gens qui éveillent des souvenirs du traumatisme.

3. Incapacité à se rappeler un aspect important du traumatisme.

4. Réduction nette de l'intérêt pour des activités antérieurement importantes ou réduction de la participation à ces mêmes activités.

$\square$ 5. Sentiment de détachement d'autrui ou bien sentiment de devenir étranger aux autres personnes.

$\square$ 6. Restriction des affects (par exemple : incapacité à éprouver des sentiments tendres).

7. Sentiment d'avenir «bouché » (par exemple : ne pas pouvoir faire carrière, se marier, avoir des enfants...).

D. Symptômes neurovégétatifs

Présence de symptômes persistants, traduisant une activation neurovégétative (ne préexistant pas au traumatisme); au moins deux des manifestations suivantes :

口 1. Difficultés d'endormissement ou sommeil interrompu

口 2. Irritabilité ou accès de colère

口 3. Difficultés de concentration

$\square$ 4. Hyper-vigilance

口. Réactions de sursaut exagérées

E. Les perturbations des critères $\mathrm{B}, \mathrm{C}$ et $\mathrm{D}$ durent plus d'un mois.

F. La perturbation entraîne une souffrance cliniquement significative ou une altération du fonctionnement social, professionnel ou dans d'autres domaines importants.

Des critères temporels sont spécifiés. Ainsi, l'ESPT aigu se caractérise par des symptômes présents sur un à trois mois, il s'agira d'un ESPT chronique quand ils existeront sur plus de trois mois. Enfin, il sera de survenue différée lorsque les manifestations débuteront six mois après l'évènement traumatique. 


\subsection{L'ETAT DE STRESS POST-TRAUMATIQUE SELON LE DSM-V}

La nouvelle édition du Manuel diagnostique et statistique des troubles mentaux (DSM-V) est parue au mois de mai 2013. Elle fait l'objet de nombreuses controverses.

Un nouveau chapitre est créé, il concernera les traumatismes et troubles liés à un facteur de stress, dans lequel on retrouvera l'ESPT avec une révision de ses critères. [69]

Le critère A1 est modifié, permettant une clarification de la notion «d'évènement traumatique » avec une précision de l'identification du facteur déclencheur en le catégorisant et en proposant des scénarii.

La personne a été exposée à un ou plusieurs des évènements suivants :

- Mort ou menace de mort

- Blessure ou menace de blessure grave

- Agression ou menace d'agression sexuelle

L'exposition résulte d'un ou plusieurs des scénarii suivant :

- Avoir personnellement vécu l'évènement

- Avoir été témoin de l'évènement

- Avoir appris que l'évènement a concerné un membre de sa famille ou un ami proche (dont la mort ou menace de mort, violente ou accidentelle)

- Exposition répétée ou extrême aux détails aversifs de l'évènement. Cela ne comprend pas les expositions au travers des médias, des images, de la télévision ou des films à moins que cela fasse partie de la profession du sujet.

Le critère $A 2$, réaction de peur intense, sentiment d'impuissance ou d'horreur, a été supprimé car jugé non prédictif de l'apparition d'un ESPT.

Plus d'attention est portée aux critères comportementaux, proposant ainsi quatre groupes diagnostics au lieu des trois du DSM-IV incluant trois nouveaux symptômes (en gras ci-dessous) : 
- La «réexpérience» du traumatisme, comprenant les souvenirs spontanés, les rêves récurrents, les flashbacks liés à l'évènement traumatique ou encore toute autre détresse psychologique intense ou prolongée induite lors d'un rappel de l'évènement par des indices externes ou internes.

- L'évitement persistant des stimuli internes ou externes associés à l'évènement.

- Les altérations cognitivo-émotionnelles marquées : la persistance d'une humeur et d'un état émotionnel négatif, des cognitions négatives sur soi et autrui, une auto dévalorisation, le sentiment d'être détaché et étranger aux autres et l'incapacité à se souvenir des aspects principaux de l'évènement.

-Les symptômes d'activation neurovégétative persistants comprenant entre autres troubles du comportement auto ou hétéro agressif, les comportements irritables ou agressifs, les prises de risque et conduites ordaliques, l'hypervigilance et les troubles du sommeil.

Les perturbations, indépendamment du déclencheur, induisent une souffrance importante, une altération des interactions sociales, de la capacité à travailler ou d'autres domaines de fonctionnement. Il ne s'agira pas de conséquences physiologiques en lien avec une autre pathologie, un médicament, une drogue ou de l'alcool.

Le nombre de symptômes devant être identifiés au sein de chaque critère dépendra du groupe auquel appartiendra le sujet.

Les critères de durée sont modifiés, la durée minimum d'un mois de manifestations symptomatiques ne sera plus nécessaire et la distinction de l'ESPT chronique et aigue ne se fera plus.

Deux formes seraient créées, l'une dite «préscolaire » afin de mieux définir l'ESPT chez les enfants de moins de 6 ans et l'autre dite «dissociative» pour les sujets présentant d'importants symptômes dissociatifs comprenant des expériences avec un sentiment de détachement de son corps ou de son propre esprit, de monde irréel, onirique ou déformé. 
Le DSM-V caractérise spécifiquement l'agression sexuelle parmi les évènements traumatiques et caractérise de façon spécifique l'ESPT chez les jeunes enfants.

Si l'on se réfère au DSM-IV-TR, de par le cadre posé par notre sujet, notre exposé porte pour l'essentiel sur l'état de stress post traumatique chronique.

\subsection{PLACE DES VIOLENCES SEXUELLES DANS LES MANUELS DIAGNOSTICS}

Au sein du DSM-IV, on peut retrouver la notion «d'abus sexuel d'un enfant » et «d'abus sexuel d'un adulte ». Les violences sexuelles ne sont alors un critère diagnostique que lorsque le motif de l'examen clinique est l'abus sexuel lui même.

Pour la CIM 10, il faut se référer aux sévices sexuels (T74.2) qui regroupent les notions d'« abus sexuels », de « sévices sexuels » et de « viol».

Ces manuels diagnostiques ont le mérite de permettre une certaine homogénéisation du vocabulaire, notamment lors de travaux de recherches. Mais ils ne doivent pourtant pas être la base de notre pratique au détriment de notre sens clinique. 


\section{EVALUATIONS DIAGNOSTIQUE ET PRONOSTIQUE DE L'ETAT DE STRESS POST-TRAUMATIQUE}

Comme dans toute spécialité médicale, avant d'établir une stratégie thérapeutique, une évaluation clinique précise est indispensable.

A la suite d'un évènement traumatique, les sujets vont présenter dans les jours qui suivent des manifestations post-traumatiques, la majorité d'entre eux va alors progressivement récupérer son fonctionnement antérieur et ne développera pas d'ESPT chronique.

Quels sont les facteurs de risque et de protection de développer un ESPT suite à des violences sexuelles? En psychotraumatologie, le ou les premiers entretiens doivent bien évidemment permettre de confirmer ou non le diagnostic, mais ils doivent surtout permettre l'investigation de ces informations satellites donnant un aiguillage pronostic et permettant de guider le patient vers les options thérapeutiques qui lui correspondent.

\subsection{EVALUATION EN DIX QUESTIONS}

Le genre demeure le plus simple des facteurs de vulnérabilité face aux violences sexuelles. Les femmes, bien que globalement moins exposées à des évènements traumatiques (Brewin et al. 2000) sont plus fréquemment victimes d'agressions sexuelles, fortement inductrices d'ESPT (Breslau et al. 2007). [29,28]

Les autres composants, moins évidents, peuvent être sondés avec comme canevas « les 10 questions de l'évaluation » proposé par Jehel L., Sylvestre M. et Louville P, (Jehel 2006). [132]

Dix questions clefs auxquelles doivent répondre les premiers entretiens, développées cidessous : 
1) Quelles sont la nature de l'évènement et l'intentionnalité humaine de l'évènement ?

Les agressions sexuelles, classées dans les évènements de type II (répétitifs et/ou infligés volontairement par un être humain) sont considérées comme de plus mauvais pronostic par rapport à une catastrophe naturelle, classée évènement de type I (imprévisible de durée limitée). Cette catégorisation des évènements traumatiques a été définie par Terr en 1991. Le caractère individuel induit une majoration de la probabilité de développer un ESPT (Norris 1992) [165].

\section{2) Quel est le délai entre l'évènement et la consultation ?}

Quand il s'agit d'un ESPT chronique, la prise en charge sera évidement différente d'un état de stress aigu et une attention toute particulière doit être portée aux comorbidités.

\section{3) Quelle est la réaction péritraumatique de la personne lors de l'évènement ?}

Les réactions péritraumatiques aussi bien physiques (tachycardie, sueurs, tremblements) qu'émotionnelles (effroi, dégoût, colère...), sont des facteurs pronostics (Ozer, 2003). [170] Ainsi, les manifestations dissociatives péritraumatiques importantes sont en général associées à un plus fort risque de développer un ESPT (Lensvelt-Mulders et al. 2008) [148].

\section{4) De quel soutien social a-t-elle bénéficié et bénéficie-t-elle encore ?}

Un soutien social précoce et étayant peut être un facteur protecteur (Cadell 2003 et Jovanovic 2004) $[35,133]$. La revue de littérature de Billette et al. en 2005, montre l'influence du soutien social sur le psychisme des victimes d'agressions sexuelles [15]. Des interactions sociales négatives sont donc de pronostic péjoratif chez les femmes victimes de violences sexuelles (Zoellner et Foa 1999) [241]

\section{5) Quels sont les antécédents psychiatriques ou d'autres évènements traumatiques ?}

Avoir présenté un trouble psychiatrique antérieur à l'évènement est un facteur de vulnérabilité. Pour Brewin (2000) [29] et Ozer (2003) [170] les troubles de la personnalité, de l'humeur, anxieux ou obsessionnels sont des facteurs de risques prétraumatiques, de même qu'un traumatisme antérieur. Ainsi, le risque de présenter un ESPT augmente avec le nombre 
et le type de traumatismes (Cougle et al. 2009) [47]. De plus, l'exposition à un évènement majore la probabilité d'expositions ultérieures (Stein et al. 2002) [198]

\section{6) Quels sont les symptômes psychotraumatiques spécifiques présents ?}

Rechercher les symptômes psychotraumatiques, en utilisant par exemple les critères du DSM, contribue à l'établissement du diagnostic et participe à l'orientation thérapeutique. Certains symptômes peuvent être la cible spécifique d'un traitement médicamenteux ou psychothérapique. Ils ont aussi bien évidemment valeur pronostic et sont évaluables à l'aide d'outils, auto ou hétéro questionnaires que nous détaillerons par la suite.

\section{7) Quels sont les autres symptômes psychiatriques, en particulier troubles} thymiques, autres troubles anxieux, conduites addictives, potentiel suicidaire ?

Kessler dans son étude de 2000 aborde l'ESPT comme un facteur de risque de présenter un épisode dépressif majeur, un abus de substance ou de conduite suicidaire [136]. Pour Shalev, Freedman et al. (1998), une symptomatologie dépressive post-traumatique serait corrélée à l'installation d'un ESPT [188]. Les troubles psychiatriques comorbides à l'ESPT se doivent d'être repérés et traités précocement. Nous les avons déjà passés en revue dans un des chapitres précédents.

\section{8) Quel est le retentissement somatique ?}

Comme nous l'avons vu dans un des chapitres précédents, ce ne sont pas uniquement des comorbidités psychiatriques que nous observons dans cette population de patients, mais aussi des pathologies somatiques, dont la fréquence interpelle. L'exposition à des évènements traumatiques notamment dans l'enfance, serait-il un facteur de risque pour certaines pathologies physiques ? Des études déjà citées ont cherché à évaluer ce lien dont «The Adverse Childhood Experiences Study » de Felitti. [78]

\section{9) Quel est le retentissement social, professionnel et personnel ?}

Tout comme les antécédents familiaux et le niveau socio-économique antérieur ont un impact sur la genèse d'un ESPT, le contexte de vie à la suite d'un évènement traumatique a de toute évidence une influence pronostic. Ainsi, dans la méta-analyse de Brewin et al. en 2000, une 
situation professionnelle difficile telle qu'une perte d'emploi peut favoriser le développement d'un ESPT. [29]

\section{0) Quelles sont les implications médico-judiciaires ?}

Au delà de la rédaction de certificat ou d'expertise, l'information et l'accompagnement des victimes sur le plan judiciaire font parties de la prise en charge et d'autant plus lorsqu'il s'agit de violences sexuelles tel qu'un viol par exemple. C'est dans ce domaine que le travail en réseau prend toute son importance. Savoir orienter ces patients vers des associations spécialisées et compétentes pour ces questions doit faire partie de la palette du professionnel de santé, afin aussi de préserver un cadre strictement thérapeutique. (Lopez 2012) [153]

Dans le guide 2007 de l'HAS, Affections psychiatriques de longue durée : trouble anxieux grave , au chapitre «traitement de l'ESPT » on retrouve l'importance du soutien psychosocial ainsi que de l'aide associative et juridique : «Le soutien psychosocial est essentiel chez la plupart des patients victimes de traumatismes graves (viols, accidents, attentats, catastrophes naturelles). L'information du patient sur son trouble et sur ses droits est essentielle, et peut être facilitée par le soutien d'associations de patients ou d'aide aux victimes (assistance juridique, psychothérapies, etc.). » [116]

Après avoir tenté de décliner les facteurs pré, péri et post-traumatiques protecteurs ou de risques de l'état de stress post traumatique il semble incontournable d'exposer les concepts de « coping » et de « résilience » 


\subsection{RESSOURCES PERSONNELLES OU COPING ET RESILIENCE}

Le terme « coping » vient de l'anglais « to cope with» ou «faire face à ». Pour Lazarus et Folkman c'est «l'ensemble des efforts cognitifs et comportementaux destinés à maîtriser, réduire ou tolérer les exigences internes ou externes qui menacent ou dépassent les ressources d'un individu ». (Jeammet P. 2004) [130]

Il regroupe les stratégies d'adaptation conscientes qu'emploient le sujet, afin de faire face à des situations déstabilisantes. Souvent utilisé pour illustrer les réactions adoptées par des patients apprenant une pathologie grave, le coping en psychotraumatologie définit les stratégies d'ajustement comme par exemple le déni, la banalisation, la colère, l'évitement, l'attitude régressive ou passive. Il semblerait que l'on entre alors dans le domaine pathologique de 1'ESPT, lorsque les capacités de coping sont dépassées ou dysfonctionnelles. Certaines thérapies cognitivo comportementales s'attachent à permettre l'acquisition de ces stratégies par le patient.

La résilience, ou capacité de certains matériaux à résister aux chocs est une notion de physique. Les premiers à l'emprunter, sont deux psychologues américaines, Werner et Smith, travaillant avec des enfants dans les années 1980, mais aussi Bowlby (1984) l'introduisant dans sa théorie de l'attachement. Il existe de multiples approches et définitions du concept de résilience en psychotraumatologie. Ainsi, pour Higgins en 1994, il s'agit de la «capacité de s'adapter malgré la présence de stresseurs ou de facteurs de risque et de se redresser après avoir vécu un évènement traumatique. » En France, ce concept ne sera introduit et vulgarisé qu'à partir des années 90, par Boris Cyrulnik (2001) : «La résilience, c'est l'art de naviguer dans les torrents » [50]. Pour Tisseron (2007) « la résilience est à la fois la capacité de résister à un traumatisme et celle de se reconstruire après lui. »

On peut ainsi considérer que chaque facteur protecteur, y compris les capacités de coping, contribue à la résilience. Certains ont cherché à la mesurer et à l'évaluer. Ainsi, Hoge et al. en ont fait une revue de littérature en 2007. [122] 
L'évaluation clinique décrite précédemment peut être aidée et complétée par des outils d'évaluation standardisés. Il existe une multiplicité d'auto et d'hétéro-questionnaires validés, nous n'en aborderons que quelques uns.

\subsubsection{Evaluation des réactions péritraumatiques}

- Le PDEQ (Peritraumatic Dissociative Experience Questionnaire) a été construit à partir du «Peritraumatic dissociative experience scale» de Mamar (1997) et validé par Birmes et Brunet (2005). Cet auto-questionnaire de 10 items mesure la dissociation péritraumatique. $[154,16]$

- Le PDI (Peritraumatic Distress Inventory), élaboré par Brunet (2001) puis traduit en français et validé par Jehel (2005), évalue avec 13 items les réactions péritraumatiques, «ce que le sujet a ressenti pendant et immédiatement après l'évènement critique ». [32,131]

\subsubsection{Instruments d'évaluation diagnostique}

- L'IES-R (Impact of Event Scale-Revised), réalisé par Weiss et Marmar (1997) à partir de l'IES d'Horowitz (1979), puis validé en français par Brunet en 2003, permet de coter avec 22 items la sévérité des symptômes post-traumatiques présents. Sans induire un diagnostic formel avec un seuil supérieur à 33, il peut néanmoins, une fois complété en cinq minutes, mettre en évidence les symptômes d'intrusion, d'évitement et d'hyperactivité neurovégétative. L'IES est l'échelle d'auto-évaluation la plus utilisée dans les travaux de recherche, entre autre d'évaluation d'efficacité des thérapeutiques. De plus, une version a été adaptée pour les enfants de plus de 8 ans. [224, 124, 33] 
- La CAPS (Clinical Administred PTSD Scale) de Blake (1990), disponible en version française, est l'instrument référence de mesure des conséquences psychopathologiques d'un évènement traumatique. Au cours d'un entretien d'environ 45 minutes, 30 questions sont soumises au patient afin d'évaluer la fréquence et l'intensité des symptômes d'ESPT selon le DSM-IV. [19]

- La PCLS (Post-traumatic stress disorder Check List Scale) comme l'indique son nom, évalue la sévérité des 17 symptômes d'ESPT décrit dans le DSM IV. Cet autoquestionnaire, validé pour les anglo-saxons par Weathers et al. en 1993, l'a été en version française en 2003 par Yao et Cottraux. Chaque item est coté par le patient, de 1 «pas du tout » à 5 «très souvent », un total supérieur à 44 semble orienter vers un diagnostic d'ESPT. $[223,232]$

\subsubsection{Recherche de troubles associés}

- Le SCID II (Structured Clinical Interview for DSM) a été élaboré à partir de la première version de Spitzer (1990). Sa passation, d'une à deux heures, doit être réalisée par un clinicien formé. Cet outil de psychopathologie générale comprend une partie d'investigation d'état de stress aigu et post-traumatique. Il permet donc la mise en évidence à la fois d'un ESPT, mais aussi d'éventuels troubles comorbides. [195]

- Le MINI (Mini International Neuropsychiatric Interview) élaboré par Sheehan (1998) et Lecrubier (1997) pour les versions anglo-saxonne et française. Les 120 questions sont plus souvent réalisées sous forme d'entretien structuré d'environ 45 minutes, mais peuvent aussi être utilisées en autoévaluation. Leur objectif est de passer en revue les troubles psychiatriques majeurs du DSM-IV. [189,146]

- Le BDI (Beck Depression Inventory), publié pour sa première version par Aaron et Beck en 1961, a été par la suite revisité afin de mieux répondre aux critères diagnostiques actuels. Cet auto-questionnaire de 21 items, est un «gold standard » de l'indentification et de l'évaluation de la sévérité d'un trouble dépressif. 
- L'HAD (Hospital Anxiety and Depression), recherche et évalue les symptômes de dépression et d'anxiété. Cet hétéro-questionnaire de 14 items a été développé par Zigmond et Snaith en 1983. D'une utilisation simple et rapide (5 minutes), il a l'avantage de pouvoir être manié par des médecins non psychiatres. [238]

- Le GHQ (General Health Questionnaire) est une auto-évaluation, présenté par Goldberg en 1972 et validé en 1979 pour le GHQ28. Traduit en français et révisé par Pariente et al. (1992), il évalue avec 28 questions la dépression, l'anxiété, le dysfonctionnement social et les troubles somatoformes. J.M. Darves-Bornoz (1998) a mis en évidence la pertinence de son utilisation chez des femmes victimes d'agressions sexuelles. [108, 109, 174, 53]

- Le SSQ (Support Social Questionnaire) de Sarason et al. 1983 recherche le nombre de personnes soutenantes et la qualité de leur soutien perçu par le patient. [186]

\subsubsection{Les outils anglo-saxons}

Nous pouvons compléter la liste précédente par deux instruments qui semblent pertinents, notamment chez des sujets victimes de violences sexuelles, mais n'existant pour l'instant qu'en anglais.

- Le PSS-SR (PTSD Symptom Scale-Self-Report) en 1993 et le PDS (PTSD Diagnostic Scale) en 1997 sont deux échelles diagnostiques élaborées en anglais par Foa et al. à partir des critères du DSM-IV. Sous forme d'auto évaluation de 49 et 14 items, elles permettent l'évaluation de la sévérité d'un ESPT. [92, 93]

- Le RAST (Rape Aftermath Symptom Test) composé de 70 items, construit par Kilpatrick en 1988 est un auto-questionnaire destiné aux victimes de viol, cherchant à évaluer les symptômes séquellaires. [138] 
Tous ces instruments d'évaluation psychométrique standardisée nous donnent une indication sur la sévérité du trouble qu'ils cherchent à évaluer, mais ils peuvent aussi faciliter l'identification par le patient de ses symptômes.

La combinaison, entretien d'évaluation complété d'auto ou d'hétéro-questionnaires, favorise une meilleure appréciation, permettant une prise en charge adaptée. L'efficacité des thérapeutiques choisies devient alors vérifiable et ce à différent temps du traitement.

Bien que valides, fidèles et sensibles pour ceux cités précédemment, ils présentent des limites. En effet, au delà d'éléments cliniques aisément évaluables car quantifiables, en cotant leur intensité ou leur fréquence, certains sont de l'ordre du subjectif, comme le sentiment de détachement d'autrui ou encore celui d'avenir bouché. Intervient alors l'incontournable évaluation personnelle du thérapeute, dépendant de son expérience clinique indispensable.

Le psychiatre ou psychologue spécialisé en psychotraumatologie n'est le plus souvent pas le premier interlocuteur du patient présentant un traumatisme psychique. Afin d'aider les professionnels non aguerris à la question, des outils simples ont été élaborés avec des guides. On peut citer une aide au repérage d'évènement traumatique en 4 questions ciblées que l'on peut retrouver sous le nom de «Trousse de diagnostic rapide» sur le site www.infotrauma.org.

Après avoir passé en revue les multiples instruments permettant de diagnostiquer les troubles post-traumatiques, il semble évident qu'il faille se pencher sur les stratégies thérapeutiques disponibles actuellement. 


\section{TROISIEME PARTIE}

\section{LES TROUBLES POST-TRAUMATIQUES CHEZ L'ADULTE : STRATEGIES THERAPEUTIQUES}


$\mathrm{Au}$ cours de l'Histoire, les procédés thérapeutiques du traumatisme ont évolué avec les guerres. Ainsi, au cours de la première guerre mondiale, les neuropsychiatres militaires tentaient de soigner le «choc des tranchées » encore appelé «obusite » par le procédé de faradisation. L'entre-deux-guerres lui, voit se développer un procédé utilisant des anesthésiants (éther, chloroforme, barbituriques, penthotal) afin de faciliter l'expression des souvenirs refoulés, la narcoanalyse.

De nos jours les thérapeutiques proposées dans la prise en charge de l'ESPT chronique, psychothérapies ou pharmacothérapies, ont pour objectif patent, l'amélioration de la qualité de vie du sujet, en diminuant sa souffrance. Les psychothérapies, qu'elles cherchent à traiter les symptômes ou le sujet traumatisé, vont pour la grande majorité inclure, dans un cadre sécurisant, un temps de rappel à la conscience du traumatisme, c'est-à-dire d'abréaction.

Quand il s'agit de victimes de traumatismes d'origine sexuelle, elles doivent avoir pour programme «de donner place dans des psychismes ravagés et désertifiés par l'effroi, à de nouveaux objets fictifs et réels sur lesquels puissent se reconstruire une histoire et un sens. » (Darves-Bornoz, 1996, P209) [52]

Aborder de manière non exhaustive les thérapies, permet de réaliser en parallèle une brève rétrospective de ces différents modèles psychopathologiques. 


\section{MODELES PSYCHOPHATHOLOGIQUES ET PSYCHOTHERAPIES DE L'ETAT DE STRESS POST-TRAUMATIQUE}

\subsection{LES THERAPIES COGNITIVO-COMPORTEMENTALES ET LES THERAPIES DERIVEES}

Le comportementalisme ou béhaviorisme est basé sur les grandes théories de l'apprentissage issues de deux expériences majeures, celle de Pavlov en 1890 et celle de Skinner dans les années quarante.

L'expérience de Pavlov est celle du «conditionnement classique ». De manière innée, la salivation du chien est déclenchée par la vue des aliments. Le conditionnement consiste à faire en sorte que le chien associe les aliments au son de la cloche, pour que par la suite le simple son de cette cloche, déclenche chez lui la salivation; théorie équivalente du «conditionnement répondant» de Watson. L'expérience de Skinner est celle du « conditionnement opérant» ou de l'apprentissage programmée par essai-erreur. Il définit alors quatre types de conditionnements opérant: le renforcement positif, le renforcement négatif, la punition positive, la punition négative.

La part cognitive se réfère au modèle de traitement de l'information encore appelé modèle cognitif de Beck. Ce dernier utilise le terme de schéma de pensée pour définir les processus de pensée conscients ou inconscients amenant notre propre interprétation du monde à travers des situations vécues ainsi que les émotions que nous y associons.

Progressivement, sur le plan thérapeutique, les deux concepts comportementalisme et cognitivisme se complètent et fusionnent en une entité : les thérapies cognitivocomportementales (TCC). Elles se développent alors depuis le milieu du $\mathrm{XX}^{\text {ème }}$ siècle. Elles se veulent issues de démarches scientifiques expérimentales et ont pour but l'apprentissage de nouveaux comportements plus adaptés, à partir de l'élaboration de pensées non ou moins dysfonctionnelles. Souvent qualifiées de thérapies de «l'ici et maintenant », les thérapies cognitivo-comportementales s'attaquent aux symptômes handicapants au quotidien le sujet et altérant sa qualité de vie. Leur principe repose sur la confrontation du patient aux situations générant chez lui de l'anxiété ou de la peur. Les stratégies thérapeutiques majeures sont la 
désensibilisation systématique, l'exposition, l'entraînement des compétences sociales, la restructuration cognitive. Les outils d'évaluation et de thérapie sont standardisés.

Le principe de déroulement des TCC peut être résumé en quatre étapes :

- Evaluation quantitative et qualitative avant le début de la thérapie avec les grilles et échelles.

- Etablissement du contrat thérapeutique avec le patient (déroulé et objectif de la thérapie)

- Mise en place de la thérapie (application du protocole)

- Evaluation des résultats

\subsubsection{Fondements théoriques des TCC dans la prise en charge de l'ESPT}

Dans le domaine des modèles comportementaux s'intéressant à l'ESPT, l'énoncé majeur est peut-être celui de Orval Hobart Mowrer en 1960. Psychologue américain à l'université de l'Illinois, il base son modèle sur le conditionnement classique et opérant. « Le traumatisme est une situation de danger, qui provoque des réactions émotionnelles (anxiété, peur), physiologiques (sursaut, hypervigilance), cognitives (danger, impuissance) et comportementales (fuite, inhibition). A cette situation initiale de danger et de peur intense, sont associés divers stimuli, qui par conditionnement vont déclencher les mêmes réponses que l'évènement traumatique. En évitant tout stimulus rappelant l'évènement traumatique, le sujet observe une diminution rapide des manifestations symptomatiques pénibles ressenties initialement. Cet apaisement momentané, recherché par le sujet, conduit à renforcer les comportements d'évitement tels que le repli social, les addictions (alcool, drogue qui permettent d'éviter les cauchemars et les troubles du sommeil) » (Pariente 2008). [173]

Modèle que l'on peut affiner avec les publications de Mac Farlane en 1988 exposant l'impact des images intrusives, ainsi que celles de David W. Foy dans les années quatre-vingt dix entre autres Cognitive Behavioral Strategy, sur les facteurs protecteurs ou fragilisants suite à une expérience traumatique. 
Pour les modèles cognitifs, il faut se référer aux travaux de Beck et ses schémas de pensée déjà cités précédemment, qui à l'origine étaient axés sur les troubles anxieux de type phobique et peu sur l'ESPT. On peut tout de même citer les travaux d'Horowitz, ou encore d'Ehlers et Clark, qui ont abouti à des modèles voulant expliquer la psychopathologie posttraumatique. Janoff-Bulman en 1992 aborde, tout comme Epstein en 1991, la modification des croyances initiales du sujet, souvent communes à tous, en croyances dysfonctionnelles suite à une situation traumatique. Citons de plus Edna Foa et Barbara Olasov Rothbaum avec leur modèle de «structure de peur » en 1998.

Les TCC se sont donc adaptées à la prise en charge de l'ESPT dans les années quantre-vingt. Il s'agit là alors d'activer le souvenir traumatique afin d'en modifier la dimension pathologique.

Plusieurs thérapies dérivées des TCC ont été développées comme thérapies spécifiques de l'ESPT notamment suite à des violences sexuelles, par exemple l'intégration neuroémotionnelle, la thérapie par l'exposition prolongée, l'EMDR (Eye Movement Desensitization and Reprocessing) et plus récemment les thérapies issues du «mindfulness » (thérapie de pleine conscience).

Je n'en exposerai que deux : la thérapie par l'exposition prolongée et l'EMDR.

\subsubsection{La thérapie par l'exposition prolongée}

Edna FOA, née à Hafai en Israël, a reçu en 1970 sa maitrise en Psychologie clinique à l'université de l'Illinois, ainsi que son doctorat en psychologie et personnalité clinique à l'université du Missouri. Actuellement professeur de psychologie clinique à l'Université de Pennsylvanie ainsi que directrice du Centre de traitement et d'étude de l'anxiété de Philadelphie, elle se consacre à l'étude des troubles anxieux. Ainsi, elle a présidé le comité du DSM-IV pour les troubles obsessionnels compulsifs et coprésidé celui pour l'ESPT. Le professeur Edna FOA a depuis publié de nombreux ouvrages et article ainsi et donné de nombreuses conférences à ce sujet. [94]

Nous exposons ici sa thérapie par l'exposition prolongée présentée lors d'un colloque en octobre 2012 «Thérapie de l'Etat de Stress Post-Traumatique par Exposition Prolongée. Soigner les victimes de viol » pour l’Université Paris-Descartes. [205] 


\subsubsection{Théorie}

La thérapie par l'exposition prolongée est basée sur le traitement des facteurs favorisant le maintien des symptômes post-traumatiques, que sont les pensées et croyances invalidantes, ainsi que l'évitement de situations mais aussi de pensées et d'images liées au traumatisme. L'évitement empêche le sujet de faire des expériences et maintient donc les croyances. D'où l'utilisation de deux types d'expositions : imaginaire et in vivo.

Les patients à qui s'adresse ce type de thérapie sont des «sujets souffrant d'ESPT chronique et psychopathologies associées à la suite de tout type de traumatisme, cela comprend les individus présentant des problèmes de comorbidités telles que dépression, autres troubles anxieux, toxicomanie, trouble de l'axe II et symptômes dissociatifs. » (Foa 2012) [94]

Elle n'a pas encore fait l'objet de publication pour des patients présentant des troubles dissociatifs de l'identité, des comportements auto-agressifs graves ou présentant une menace imminente de suicide ou encore chez des patients psychotiques.

\subsubsection{En pratique}

Le programme se déroule en 10 à 12 séances hebdomadaires de 90 minutes chacune. Ces séances regroupent quatre grands principes :

- Identification et explication au patient de ses symptômes et de ses réactions issues de son traumatisme que l'on va chercher à diminuer.

- L'enseignement de la rééducation respiratoire (ralentir la respiration et se concentrer sur l'expiration lente)

- L'exposition en imagination : le patient faisant le récit, les yeux fermés, se replonge dans l'évènement traumatique en y associant les émotions ressenties. Le thérapeute fait preuve d'empathie tout en faisant le moins de commentaire possible.

- L'exposition réelle : le patient se confronte de manière répétée aux situations craintes et évitées depuis l'évènement.

Chaque séance se termine par un «travail à la maison » qui sera alors le point de départ de la suivante. Elles sont enregistrées par vidéo ou audio, le patient devant les réécouter au moins une fois dans la semaine avant la suivante. Comme outils, E. Foa conseille d'utiliser des questionnaires dont le QAPP (Questionnaire sur l'Agression et le Passé du Patient) et le PSS (PTSD Symptom Scale). 


\subsubsection{Déroulé des séances d'exposition prolongée simple dans le cas d'un viol [94] \\ Séance1}

- Présentation de la thérapie (25-30 min) :

- Quelles sont les procédures utilisées dans le cadre du programme

- Expliquer l'importance accordée aux symptômes de l'ESPT

- Recueillir des informations relatives à l'agression grâce au QAPP (45 min)

- Présenter le contrôle respiratoire (10-15 min)

- Donner les exercices à faire à la maison :

- Pratiquer le contrôle respiratoire 10 min trois fois par jour

- Lire le document «Raison d'être de la thérapie »

- Ecouter l'enregistrement de la séance une fois

\section{Séance 2}

- Discussion sur le travail réalisé à la maison (5 min)

- Présentation du programme de la séance (5 min)

- Utilisation de la «fiche sur les réactions courantes aux agressions » pour informer le patient des symptômes de l'ESPT (25 min)

- Explication de l'exposition in vivo (10 min)

- Présentation des unités subjectives de détresse ressentie (SUD) (5 min)

- Construction d'une hiérarchie des situations in vivo (20 min)

- Choix d'exercices in vivo à faire à la maison (5 min)

- Donner les exercices à faire à la maison (10 min)

- Lire quotidiennement la « fiche sur les réactions courantes aux agressions »

- Continuer les exercices de contrôle respiratoire

- Lire la liste des situations évitées à la maison et la compléter

- Commencer le travail d'exposition in vivo

- Ecouter au moins une fois l'enregistrement de la séance.

\section{Séance 3}

- Discussion sur le travail réalisé à la maison (10 min)

- Présentation du programme de la séance (5 min)

- Exposition imaginaire (60 $\mathrm{min})$

- Présenter l'exposition

- Mener l'exposition

- Traiter l'exposition 
- Donner les exercices à faire à la maison (10 min)

- Continuer les exercices de contrôle respiratoire

- Ecouter au moins une fois par jour l'enregistrement de l'exposition imaginaire

- Continuer les exercices d'exposition in vivo quotidiennement, en remontant les niveaux de la hiérarchie des SUD

- Ecouter au moins une fois l'enregistrement de la séance

\section{Séances 4 à 8}

- Discussion sur le travail réalisé à la maison (10 min)

- Présentation du programme de la séance (5 min)

- Mener et traiter l'exposition imaginaire : en mettant progressivement l'accent sur les points sensibles (30-45 $\mathrm{min})$

- Discuter puis mettre en application l'exposition in vivo (20 min)

- Donner les exercices à faire à la maison (10 min)

- Continuer les exercices de contrôle respiratoire

- Ecouter au moins une fois par jour l'enregistrement de l'exposition imaginaire

- Continuer les exercices d'exposition in vivo quotidiennement

- Ecouter au moins une fois l'enregistrement de la séance

- A la huitième séance, utiliser la PSS et déterminer si un traitement supplémentaire est nécessaire.

\section{* Dernière séance}

- Discussion sur le travail réalisé à la maison (10 min)

- Présentation du programme de la séance (5 min)

- Exposition imaginaire (30 $\mathrm{min})$

- Traiter l'exposition et évaluer avec le patient la mesure dans laquelle sa vision du traumatisme a changé

- Bilan détaillé des progrès réalisés et suggestion pour continuer les exercices (30 min)

- Mettre fin à la thérapie, discuter d'un suivi et conseiller de poursuivre le travail personnel.

Pour certains patients, présentant colère, honte ou culpabilité, on peut inclure dans ces séances des exercices de restructuration cognitive, ayant pour but d'identifier et de remettre en question les distorsions cognitives. 


\subsubsection{Eye Movement Desensitization and Reprocessing (EMDR)}

L'EMDR, que l'on peut traduire en français par désensibilisation et retraitement des informations par mouvements oculaires, a été développé par Francine Shapiro depuis la fin des années quatre-vingt, comme thérapie spécifique du stress post-traumatique. Francine Shapiro est docteur en psychologie au Mental Research Institute de Palo Alto aux USA.

Sa technique a, dans un premier temps, été expérimentée chez les vétérans de guerre comme la plupart des autres thérapies s'intéressant à l'ESPT, puis s'est étendue aux prises en charges d'autres types de traumas, notamment chez les sujets victimes d'agressions sexuelles. Le premier article publié par Shapiro date de 1989: "Efficacy of the eye movement desensitization procedure in the treatment of traumatic memories" dans Journal of Traumatic Stress décrivant son efficacité. Elle connait un véritable essor depuis les années 2000 aux USA ainsi qu'en Europe.

Cette thérapie n'est pas une TCC à proprement parlé car elle fonctionne avec un protocole qui lui est propre et fait appel à des connaissances en neurologie. Cependant, elle intègre tout de même des modèles cognitivistes avec entre autres la notion de «distorsion cognitive » ainsi que des hypothèses comportementalistes dont «l'équilibre excitation-inhibition du cerveau normal » de Pavlov.

\subsubsection{Principe}

La thérapie du Dr Shapiro est basée sur le constat personnel suivant : des mouvements oculaires abaissent son seuil d'anxiété en atténuant ses pensées négatives et ses ruminations. Elle va alors développer un protocole visant à utiliser les stimulations sensorielles bilatérales alternées oculaires afin de modifier les souvenirs négatifs anxiogènes de l'évènement traumatique qui ont été créés sous forme sensorielle et non cognitive par le cerveau. Les mouvements oculaires, proches des mouvements spontanés de nos yeux au cours des rêves, vont mobiliser l'attention du sujet alors qu'il s'expose aux souvenirs qu'il a de l'évènement. Le système limbique, que nous savons depuis Papez (1939) impliqué dans les comportements émotionnels, les apprentissages et la mémoire, est alors stimulé en activant de manière alternative les deux hémisphères cérébraux, ce qui permet au sujet au cours de cet état de relaxation, d'intégrer différemment les informations liées au trauma. 


\subsubsection{Déroulement de la thérapie}

Les premières séances (les deux premières en général) sont celles de l'évaluation et de l'établissement de l'alliance thérapeutique. Ces premiers entretiens permettent de poser la réelle indication de l'EMDR en définissant l'évènement cible, mais aussi d'identifier la complexité du traumatisme, la souffrance, les symptômes invalidants, ainsi que les comorbidités que peut présenter le patient. Le patient doit de plus identifier ses ressources positives et ainsi définir son «lieu sûr », lieu en imagination où il se sent en sécurité. Le thérapeute pourra l'utiliser dans les cas où le patient demande à interrompre le protocole par un «signal d'arrêt ».

Assis en face à face, la séance d'environ une heure débute par le choix de la scène cible par le patient avec l'aide du thérapeute. Puis il lui est demandé d'identifier une ou deux croyances négatives, d'y associer les croyances positives inverses, que le sujet note par une échelle de véracité allant de 1 à 7 . Il doit aussi noter les émotions rattachées à la scène sur une échelle de perturbation de 1 à 10 .

S'en suit donc l'utilisation des mouvements oculaires par épisode de trente secondes à quelques minutes. Alors que ses pensées et ses émotions ressurgissent, le patient suit des yeux, les mouvements alternatifs et brefs qu'effectue le thérapeute avec ses doigts. Entre les épisodes, le sujet verbalise son ressenti, le thérapeute n'en fait aucune interprétation et intervient peu.

Les séries de balayage se poursuivent tant que les émotions ne sont pas maîtrisées ou positives. L'objectif idéal est d'obtenir une échelle de véracité de 7 et de perturbation de 0 .

A la fin de la séance le sujet réalise un «scan corporel» afin de détecter toute tension ou émotion résiduelle en lien avec la cible qui pourrait alors servir de point de départ lors de la suivante.

Le nombre total de séances nécessaires peut être très variable mais est en général de 4 à 6 .

Un livret explicatif des protocoles d'EMDR est consultable sur :

http://www.barbery.net/psy/emdr/emdr.pdf 
Pour l'instant, le rôle réel des mouvements oculaires n'est pas clairement expliqué sur le plan théorique. Certains utilisent d'autres stimulations sensorielles le toucher (par des tapotements alternatifs sur les mains du patients ou encore des vibrations), l'audition (par des sons sollicitant de manière alternative l'oreille droite puis gauche), ou encore le visuel (mais cette fois en utilisant la lumière).

Très récemment on observe l'essor de l'Intégration par les Mouvements Oculaires (IMO). Thérapie dérivée de l'EMDR, mise en place par Steve Andreas et Connirae au Colorado, puis développée par une psychologue québécoise, Danie Beaulieu. L’IMO utilise aussi des mouvements oculaires mais d'un autre type, le SPEM (Smooth Pursuit Eye Mouvement) ou poursuite visuelle continue.

L'EMDR est reconnue efficace dans le traitement de l'ESPT, en France depuis 2004 par l'Inserm (Institut national de la santé et de la recherche médicale) dans son rapport Psychothérapies trois approches évaluées ainsi que par l'American Psychiatric Association et le National Institue of Clinical Excellence du Royaume Uni depuis 2005. En juillet 2012, l'Organisation Mondiale de la Santé lors de sa réunion scientifique à Amman, a officiellement reconnue l'EMDR comme l'une des thérapies recommandées pour le traitement de l'ESPT. Elle bénéficie aussi de recommandations de l'HAS (Haute Autorité de Santé) depuis 2007 dans Traitement de troubles anxieux graves. [116]

Ces thérapies types TCC et leurs dérivées ont une efficacité sur la qualité de vie immédiate des patients souffrant de troubles post-traumatiques qui ne peut être discutée. Cependant, leurs limites résident entre autres dans la capacité du sujet à avoir au préalable identifié les symptômes handicapants et à être conscient que certains stimuli peuvent être déclencheurs de souvenirs traumatiques. Certaines études vont dans le sens d'une efficacité limitée. Pour certains, TCC et EMDR auraient un taux de rechute de 30 à 40\% à un an (Vaiva et al., 2008) [215].

Les arguments fréquemment retrouvés en opposition aux TCC sont la standardisation des protocoles, accompagnés d'une volonté de normalisation ainsi que la réduction de la relation médecin malade à l'alliance thérapeutique, sans en analyser les mouvements transférentiels et en chercher le sens. 


\subsection{LES PSYCHOTHERAPIES PSYCHODYNAMIQUES}

Les thérapies psychodynamiques regroupent aussi bien la psychanalyse traditionnelle que les psychothérapies dites d'inspiration analytique longues ou brèves.

Pour aborder ces thérapies dans le cadre de l'ESPT, il faut exposer le modèle d'Horowitz qui trouve ses bases sur la théorie freudienne, mais qui reprend aussi la notion de traitement de l'information. Selon lui, un blocage ou un défaut de «perlaboration » va induire chez le sujet les troubles. Ces troubles peuvent entre autres se manifester par des réactions psychosomatiques ainsi que des comportements inadaptés.

Horowitz a formalisé sa psychothérapie psychodynamique brève en vingt séances, visant la résolution de conflits intrapsychiques. Ceux-ci inhibant le processus d'adaptation du sujet depuis le traumatisme vécu. La position du thérapeute au cours de cette thérapie est différente de celle de la cure analytique. Les principes de «neutralité bienveillante » et «d'attention flottante » sont relégués au profit d'une attitude empathique ainsi qu'un rôle actif du thérapeute. Celui-ci mène les entretiens, guide le sujet vers la prise de conscience de l'altération, depuis le trauma, des conceptions antérieures de soi et de sa perception de son environnement. Il accorde une place toute particulière à l'analyse du contre-transfert. Les modalités de cette thérapie ont la particularité d'intégrer des recommandations médicamenteuses mais aussi concernant le sommeil, l'alimentation, les loisirs, la relaxation, l'incitation aux liens sociaux et aux groupes de soutien, les réactions et positionnement des proches.

Selon la théorie psychodynamique, la réponse au traumatisme du viol peut être expliquée de la manière suivante : «On peut dire que le hiatus entre les informations réelles apportées par l'évènement traumatique et la conception du soi antérieure au traumatisme est ce qui produit le stress traumatique. Autrement dit le viol est traumatique parce qu'il contredit les conceptions, parfois naïves et sûres d'elles mêmes, sur le soi et le monde qui assureraient un équilibre serein de la personne. C'est une blessure narcissique qui ne peut cicatriser que dans un processus de transformation du soi, comme dans un autre domaine une perte ne peut cesser de provoquer de la souffrance qu'avec une réorganisation du soi » (Darves-Bornoz 1996, P199) [52] 
La limite de ces thérapies d'inspiration psychanalytique peut venir de la nécessaire grande capacité d'introspection du patient. De plus elles sont peu pratiquées par les analystes qui par attachement à leur pratique traditionnelle sont peu habitués à la position active. La cure analytique elle, est décrite comme peu adaptée à ce type de patients, qui souvent de par la nature même de leur traumatisme psychique, perçoivent la position du psychanalyste comme agressive voire critique.

Nous aborderons dans l'un des chapitres qui suit, un nouvel outil de la palette des psychothérapies psychodynamiques brèves, la thérapie interpersonnelle.

\subsection{L'HYPNOSE}

Selon l'American Psychological Association, l'hypnose est une «procédure spécifique au cours de laquelle on suggère au client d'expérimenter des changements au niveau de ses sensations de ses perceptions, de ses pensées et ou de ses comportements ». Charcot, Freud et Janet déjà, utilisaient l'hypnose au cours de leurs travaux sur l'hystérie et le trauma. La dissociation selon Janet nous permet de comprendre que l'hypnose est une forme de dissociation thérapeutique.

L'hypnose traditionnelle telle que la pratiquait Freud, utilisant la suggestibilité, est contre indiquée dans les traumatismes notamment d'ordre sexuel, car sous-tend une certaine emprise du thérapeute sur le sujet. Diverses méthodes d'hypnothérapie ont alors été développées, l'approche ericksonienne étant l'une des références dans le domaine du psychotraumatisme.

Milton Erickson, psychiatre américain (1901-1980), a beaucoup travaillé, avec une conception différente de l'Inconscient, sur l'hypnose comme thérapeutique. Il définit l'hypnose comme «l'évocation et l'utilisation des apprentissages inconscients ». Pour lui, l'Inconscient n'est pas une menace pulsionnelle mais le contenant d'un ensemble de compétences. Capacités qui, une fois identifiées et mobilisées par le sujet, peuvent lui permettre de résoudre ses problèmes. Selon Erickson : «chaque personne est unique et ne peut entrer dans aucune théorie ». Il faut alors «créer des situations dans lesquelles l'individu modifiera lui-même volontairement sa façon de penser. » (Zeig 1985) [236] 
Ainsi, l'actuelle pratique de l'hypnose ericksonienne, développée en 1937 par ses élèves Jay Haley, Jeffrey Zeig et Ernest Laurence Rossi, s'inspire de ses observations et théories. Réalisée sur trois à dix séances, elle se veut être une pratique interactionnelle et non standardisée, au cours de laquelle le thérapeute utilise les données apportées par le patient pour le guider dans sa prise de conscience de ses ressources intérieures.

E. Cardeña et D. Spiegel quant à eux ont développé une approche d'hypnose en huit étapes, praticable dans le traitement suite à une agression sexuelle (Cardeña 2000) [36] .

1- Confronter le traumatisme : on demande au patient de reconnaître l'importance du trauma dans l'étiologie de ses symptômes.

2- Condensation du traumatisme : trouver une condensation de l'expérience traumatique, un moment particulier, effrayant du psychotraumatisme.

3- Confession à soi-même : reconnaître, en revoyant ou revivant les expériences traumatiques, les sentiments ou les situations dont ils se sentent profondément honteux, qu'ils n'ont jamais osé raconter à personne.

4- Consolation : le thérapeute peut exprimer son émotion aux patients et les «consoler»de façon appropriée par des mots, des recadrages, des éclairages, des gestes d'encouragement et des sentiments sincères qu'il éprouve pendant la séance.

5- Conscience : rendre conscients des évènements réprimés ou dissociés entraîne la possibilité de les restructurer. Prendre conscience d'un certain nombre de ressources dont le patient n'avait plus conscience en raison de sa souffrance.

6- Concentration : mobiliser et focaliser la concentration. Cela permet d'activer des souvenirs et de les désactiver quand le travail thérapeutique est terminé.

7- Contrôle : le traumatisme, par ses conséquences, entraîne un sentiment d'impuissance chez le patient. La thérapie par l'hypnose améliore le contrôle des émotions intimement liées au souvenir des expériences présentes et passées.

8- Congruence : le but est d'aider à affronter cette période tragique, à l'intégrer dans la vision présente qu'ils ont désormais d'eux-mêmes, afin que les souvenirs n'aient plus besoin d'être dissociés pour survivre. Ceci implique une restructuration du souvenir afin de le rendre plus supportable à l'état de conscience. (Simon 2006) [193] 
Concernant la réelle efficacité de ce type de thérapies, très peu d'études ont été menées, on retrouve essentiellement des études de cas. La publication de Brom et al. en 1989 est à citer, nous l'aborderons dans l'un des chapitres qui suit. [31]

La limite majeure à son utilisation est la susceptibilité hypnotique du sujet. Il s'agit de la capacité de l'individu à répondre à une série de suggestions au cours de la procédure. Les patients ayant vécu un traumatisme présentent assez fréquemment des symptômes de dissociation, ils sont alors particulièrement hypnotisables. De plus, aussi bien sur le plan thérapeutique qu'éthique, elle ne doit absolument pas être pratiquée avec l'objectif de retrouver des souvenirs traumatiques. Le risque est alors d'engendrer de « faux souvenirs ».

En pratique, on comprend alors que l'hypnose trouve tout son sens dans la prise en charge de patients présentant un PTSD, entre autre dans les suites de violences sexuelles, quand elle est utilisée en synergie avec les autres thérapeutiques, quelles soient de type cognitivocomportementale, psychodynamique ou encore médicamenteuse. 


\section{COMPREHENSION DE LA NEUROPHYSIOLOGIE DU STRESS ET LA PHARMACOTHERAPIE DE L'ESPT}

La pharmacothérapie aura pour cible l'amélioration des symptômes propres de l'ESPT mais aussi la prise en charge des comorbidités tel qu'un syndrome dépressif ou encore un trouble anxieux.

Envisager les traitements médicamenteux oblige d'expliciter les hypothèses neurobiologiques de l'ESPT pour ainsi permettre la compréhension leur mode d'action. (Cheiwess 2000, Elias 2001) $[40,77]$

\subsection{LES MECANISMES NEUROBIOLOGIQUES}

D'un point de vue neurologique, l'ESPT serait associé à une hyperactivité amygdalienne par défaut d'activation du cortex préfrontal médian ainsi qu'un dysfonctionnement hippocampique avec diminution de son volume en lien avec une altération du traitement de la mémoire contextuelle (Shin et al. 2001) [190]. Dysfonctionnements associés à une dysrégulation de plusieurs hormones et neurotransmetteurs.

La physiologie normale du stress a pour axe le système hypothalamo-hypophyso-surrénalien. Une situation de stress va stimuler la libération par l'hypothalamus de CRH (Corticotropin Releasing Hormone), celle-ci stimule la libération d'ACTH (Adreno Cortico Trophic Hormone) par l'hypophyse, qui va alors induire la sécrétion de cortisol par la corticosurrénale mais aussi de catécholamines (adrénaline, noradrénaline) par la médullo-surrénale. Le cortisol permet un rétrocontrole négatif sur l'axe hypothalamo-hypohysaire et intervient dans la régulation d'autres neuromédiateurs. Cortisol, adrénaline et noradrénaline par des mécanismes complexes, ont une action sur le locus coeruleus, les amygdales et les hippocampes.

Un dysfonctionnement de l'axe corticotrope est constaté chez les patients présentant un ESPT : une diminution de la cortisolurie, une cortisolémie moyenne plus faible au cours du nycthémère, une augmentation de la sensibilité des récepteurs aux glucocorticoïdes ainsi qu'une hyperfreination de l'axe corticotrope (Yehuda R. et al. 1991, 1993) [233,234]. 
Cependant, la chronologie de ces dérèglements neurobiologiques chez des patients présentant un ESPT est encore incertaine. Sont-ils antérieurs au trauma et dans ce cas ils seraient un facteur de vulnérabilité, ou bien sont-ils secondaires?

\subsection{LES ANTIDEPRESSEURS}

Dans les suites d'un évènement traumatique, la majorité des symptômes évoluent naturellement de manière positive dans la plupart des cas, l'instauration du traitement ne doit donc pas se faire dans le premier mois. La période d'évaluation d'efficacité d'une molécule pour un patient donné est de six semaines. Le traitement médicamenteux, une fois jugé efficace, sera d'une durée minimale de 12 mois. (Attal, 2009) [8]

\subsubsection{Les inhibiteurs de la recapture de la sérotonine}

Les inhibiteurs de la recapture de la sérotonine (ISRS) sont les molécules références des recommandations internationales dans l'ESPT comme celles de la World Federation of Societies of Biological Psychiatry (WFSBD) en 2002. [230]

Leur mode d'action est basé sur la sérotonine modulant l'hyperstimulation du circuit de la peur, régulant alors le système noradrénergique du locus coeruleus.

Les publications montrant leur efficacité sont pour la Fluoxétine (PROZAC®), Connor et al 1999, Van Der Kolk et al. 1994 [46,217], pour la Paroxétine (DEROXAT®) Tucker et al. 2001 [210], pour la Sertraline (ZOLOFT®) Brady et al. 2000, Davidson et al. 2001, Zohar et al. 2002 [25, 56, 240] et pour le Citalopram (SEROPRAM®), Tucker et al. 2003 [211].

En France, seules la Paroxétine et la Sertraline (depuis mars 2013, [118]) ont l'autorisation de mise sur le marché avec cette indication. Les ISRS montrent alors tous leurs intérêts en cas de troubles associés tel que dépression, addiction, attaque de panique ou trouble obsessionnel. 


\subsubsection{Les inhibiteurs de la monoamine oxydase et les tricycliques}

Pour les IMAO, (inhibiteurs de la monoamine oxydase), seule la Phenelzine, non commercialisée en France, a fait l'objet d'évaluations. Certaines montrent son efficacité en la comparant à l'Imipramine (Frank J.B.et al 1988 ; Kosten et al 1991). [98,142]

Pour les antidépresseurs tricycliques, parmi de très nombreuses publications, deux montrent une amélioration des symptômes d'ESPT sans symptomatologie dépressive. Davidson et al. 1990 pour l'Amitriptiline (LAROXYL®) et Crocq 1992 pour l'Amoxapine (DEFANYL®). $[54,48]$

Tricycliques et IMAO sont peu utilisés car ils ont une moins bonne tolérance que les ISRS.

\subsubsection{Les autres antidépresseurs}

Concernant la Mirtazapine (NORSET®), l'étude de Davidson de 2003 conclut à son efficacité sur les symptômes d'ESPT mais aussi sur les troubles du sommeil. [55]

Parmi les IRSNA (Inhibiteurs de la Recapture de la Sérotonine et de la Noradrénaline), la Venlafaxine (EFFEXOR®) a fait l'objet de publications dont celle de Davidson en 2006. [57]

La prescription de ces deux molécules est recommandée en seconde intention en cas de non efficacité des ISRS.

L'Agomélatine (VALDOXAN®), dont le mode d'action est à la fois agoniste de récepteurs de la mélatonine et antagoniste des récepteurs 5HT2c de la sérotonine, ne bénéficie pour l'instant dans le cadre de l'ESPT que de publication d'étude de cas (De Berardis 2012). [60] 


\subsection{LES THYMOREGULATEURS}

L'action des anticonvulsivants tiendrait de leurs propriétés «anti-kingling », théorie empruntée au modèle de l'épilepsie. L'exposition répétée à des stimuli négatifs (symptômes de répétition et reviviscences) entraînerait une baisse du seuil d'excitabilité et donc une majoration de la réactivité neuronale. Les anticonvulsivants réguleraient cette hyperréactivité neuronale, limiteraient les symptômes intrusifs et l'irritabilité du sujet.

Les études dans le cadre de l'ESPT sont multiples :

- Ainsi, la supériorité de la Lamotrigine (LAMICTAL®) est montrée face à un placebo (Hertzberg et al 1999) [120].

- Fesler (1991) conclut à l'amélioration des symptômes d'hypervigilance avec le Valproate (DEPAKINE®) [84].

- Quant au Valpromide (DEPAMIDE®), lui a l'AMM en France pour les troubles caractériels et les états d'agressivité mais reste peu étudié car non commercialisé aux Etats-Unis.

- La Carbamazépine (TEGRETOL®) est utile pour l'impulsivité et l'irritabilité mais aussi pour les troubles du sommeil (Lipper 1990 et Schoenfeld 2004) [152, 187].

Ducrocq F., Vaiva et al. en 2001 mettent en avant l'efficacité du Lithium, du Valproate, de la Carbamazepine, de la Gabapentine (NEURONTIN®) et de la Lamotrigine. [70]

Le Lithium aurait un intérêt chez les patients manifestant une impulsivité et une agressivité marquées (Forster 1995). [96]

En pratique les thymorégulateurs, anticonvulsivants et lithium, sont majoritairement utilisés en association aux antidépresseurs et beaucoup moins en première ligne. 


\subsection{LES ANTIPSYCHOTIQUES ATYPIQUES}

Les symptômes d'hypervigilance, d'anxiété et de sursaut sont pour certains rattachés à l'hyperactivité du système dopaminergique dans l'ESPT (Friedman 1998) [103]. Cette hypothèse justifie alors l'utilisation de l'action antagoniste dopaminergique des antipsychotiques.

Leurs bénéfices comme traitement adjuvant sont montrés dans les études entre autres de Bartzokis (2005) pour la Risperidone (RISPERDAL®), Stein (2002) pour l'Olanzapine (ZYPREXA®) mais aussi Kozaric-Kovacic (2007) et Hammer M.B. (2003) pour la Quétiapine (XEROQUEL®). [13,199,143,115]

Tout comme les thymorégulateurs classiques, les antipsychotiques sont plus facilement utilisés chez des patients présentant des troubles du comportement associés.

\subsection{LES ANXIOLYTIQUES}

Actuellement, la prescription des benzodiazépines est sujette à controverse, aussi bien en phase aigue après un trauma que pour l'ESPT chronique. En effet, les résultats des études sont contradictoires et iraient plutôt dans le sens d'un effet délétère notamment de l'Alprazolam (XANAX®) (Braun 1990 [26]). Si l'on doit avoir recours à cette classe de médicaments, il est préférable de prescrire une benzodiazépine à demi vie longue, afin d'éviter le phénomène de rebond anxieux mais surtout de manière très ponctuelle, pour éviter le risque de dépendance, accrue dans cette population de patients.

Il existe des alternatives intéressantes avec d'autres classes d'anxiolytiques. La Buspirone (BUSPAR $\left.{ }^{\circledR}\right)$ qui bénéficie de bons résultats dans le trouble anxieux post-traumatique (Duffy, 1994 [74]) mais aussi, l'Hydroxysine (ATARAX®), dont l'intérêt est surtout en phase aigue. Les antihistaminiques $\mathrm{H} 1$, classe dont fait partie l'Hydroxysine, bloquent la voie histaminergique au niveau hippocampique ce qui empècherait la constitution des phénomènes de conditionnement. De plus ils contrôleraient les effets sur l'amygdale de l'hyperactivité adrénergique. D'autres antihistaminiques H1 comme la Cyproheptadine ont fait l'objet d'observations sur les cauchemars récurrents (Gupta, 1998) [113]. 


\subsection{EN RESUME}

L'option médicamenteuse reste une thérapeutique adjuvante aux psychothérapies. Pour certains elle faciliterait le travail psychique. (Lebigot 2006) [145]

Son instauration ne peut se faire qu'après une évaluation minutieuse comprenant les antécédents, le contexte de vie, les troubles associés ainsi que les bénéfices attendus. La prescription s'accompagne bien évidemment de l'information au patient avec entre autre l'éducation thérapeutique favorisant la bonne observance. L'action des différentes classes est résumée dans le tableau 4.

Tableau 4: Impacts des principaux psychotropes sur les principaux symptômes de l'ESPT

\begin{tabular}{|c|c|c|c|c|c|c|}
\hline Symptômes cibles & ISRS & IMAO & Tricycliques & Benzodiazépines & Bétabloqueurs & Anticomitiaux \\
\hline $\begin{array}{l}\text { Réduction du syndrome de } \\
\text { répétition }\end{array}$ & ++ & ++ & $+/ 0$ & + & ++ & + \\
\hline $\begin{array}{l}\text { Réduction de la tendance à } \\
\text { tout interpréter comme une } \\
\text { récurrence du traumatisme }\end{array}$ & ++ & + & + & 0 & ++ & + \\
\hline $\begin{array}{l}\text { Réduction de l'état } \\
\text { d'hyperéveil }\end{array}$ & + & + & + & ++ & + & 0 \\
\hline Réduction des évitements & + & ++ & + & + & $?$ & + \\
\hline $\begin{array}{l}\text { Amélioration de l'état } \\
\text { dépressif et de } \\
\text { l'émoussement affectif } \\
\text { (numbing) }\end{array}$ & $\begin{array}{l}\text { Dépression : + } \\
\text { Numbing : } 0\end{array}$ & $\begin{array}{l}\text { Dépression : }+ \\
\text { Numbing : } 0\end{array}$ & + & 0 & - & + \\
\hline $\begin{array}{l}\text { Réduction des éléments } \\
\text { dissociatifs }\end{array}$ & 0 & 0 & & 0 & 0 & + \\
\hline $\begin{array}{l}\text { Réduction de l'agréssivité } \\
\text { (auto- et hétéro) }\end{array}$ & + & + & & + & + & ++ \\
\hline
\end{tabular}

Source : Neurobiologie des états de stress post traumatiques Actualité en neurologie, Septembre 2000

L.Cheiwess [40] 


\section{LES THERAPEUTIQUES NOVATRICES}

\subsection{LES STIMULATIONS MAGNETIQUES TRANSCRANIENNES (r-TMS)}

La première description de stimulations magnétiques du cortex cérébral date de 1896 par Arsène d'Arsonval. La première étude clinique elle, de 1985 en Angleterre par Anthony Barker et son équipe. Son principe est non invasif, il s'agit d'appliquer grâce à une bobine d'induction placée sur le scalp, un champ magnétique, qui réalisera une stimulation ciblée de réseaux neuronaux corticaux.

Les premières applications et études cliniques en psychiatrie datent des années 90 et s'inscrivaient surtout dans le cadre du traitement de la dépression.

Dix méta-analyses entre 2001 et 2010 concluent à l'efficacité des TMS pour la dépression. Aujourd'hui, ses indications en psychiatrie sont la dépression résistante et les hallucinations résistantes chez les schizophrènes. En neurologie, elle est appliquée dans le cadre de maladie de Parkinson, de douleurs chroniques comme la fibromyalgie ainsi que suite à des accidents vasculaires cérébraux. Les TMS présentent peu d'effets cognitifs délétères, elles se pratiquent en ambulatoire, sans anesthésie, le patient restant conscient.

Les contre indications concernent les femmes enceintes, les porteurs de pacemaker, d'implants métalliques magnétiques et les sujets ayant des antécédents de comitialité de traumatisme crânien, d'hypertension intracrânienne ou de neurochirurgie.

Les études récentes, souvent couplées à l'imagerie fonctionnelle, cherchent à étendre ces indications, par exemple dans le cadre d'addictions, de troubles anxieux et de l'ESPT.

Dans ce sens, Cohen, Kaplan et leurs collaborateurs ont mené en Israël une étude publiée dans 1'American Journal of Psychiatry en 2004, «Repetitive Transcranial Magnetic Stimulation of the right dorsolateral prefrontal cortex in Posttraumatic Stress Disorder : a double-blind, placebo-controlled study ». [44] Vingt-quatre patients souffrant d'ESPT ont été randomisés en trois groupes pour recevoir, soit une basse fréquence $(1 \mathrm{~Hz})$, soit une haute fréquence $(10 \mathrm{~Hz})$ ou de fausses TMS. Les TMS étaient administrées en double aveugle, dix séances quotidiennes, sur quinze jours avec une évaluation avant, pendant et après le 
protocole, de la sévérité des symptômes d'ESPT, de dépression et d'anxiété. Les résultats montraient des effets plus thérapeutiques pour le groupe bénéficiant des TMS à $10 \mathrm{~Hz} d u$ cortex préfrontal dorsolatéral droit, que pour les groupes basse fréquence ou stimulation factice, avec une amélioration nette des symptômes d'ESPT tels que évitement et reviviscence.

\subsection{LA THERAPIE INTERPERSONNELLE}

La thérapie interpersonnelle, approche psychodynamique brève, a initialement été développée aux Etats-Unis dans les années 70 par G. Klerman et M. Weissman pour le traitement de la dépression. L'étude des relations interpersonnelles de Sullivan, la psychobiologie de Meyer et la théorie de l'attachement de Bowlby en sont les trois bases théoriques.

Encore très peu pratiquée en France, son efficacité dans la dépression fait l'objet de publications, dont celle de Cuijpers et al. en 2011. [49]

Théoriquement structurée sur 12 à 16 séances, elle travaille sur quatre dimensions liées à la dépression. Le deuil pathologique, les conflits interpersonnels, les déficits interpersonnels et la transmission difficile (difficultés en lien avec un changement de statut).

Actuellement, la thérapie interpersonnelle fait l'objet d'élargissement de son champ d'application et notamment dans l'ESPT. En effet, les quatre thèmes cités précédemment peuvent être retrouvés comme problématique dans l'ESPT notamment après une agression sexuelle.

Ainsi, J.L Krupnick a développé une thérapie interpersonnelle de groupe spécifique pour les victimes d'agressions sexuelles. [144]

Deux études récentes de faible puissance (Bleiberg 2005, Robertson et al. 2007) retrouvent une efficacité intéressante de la thérapie interpersonnelle dans le traitement de l'ESPT. $[21,181]$ 


\subsection{NOUVELLES OPTIONS PHARMACOLOGIQUES}

\subsubsection{Les agents adrénergiques}

Une augmentation de l'activité du système noradrénergique semble en lien avec les symptômes comme l'hypervigilance, l'insomnie, les cauchemars et reviviscences ainsi que les attaques de panique (Yehuda R. et al. 1991), d'où l'utilisation possible d'agents adrénolytiques qui inhiberaient l'activité du locus coeruleus. [233]

Les études sur la Prazosine (ALPRESS $®)$, qui est un alpha 1 adrénergique, montrent un effet significatif sur les troubles du sommeil (Dierks 2007). [66]

La Clonidine (CATAPRESSAN®), agoniste alpha 2 adrénergique, diminuerait les cauchemars et les réactions de sursaut chez les vétérans de guerre (Kolb 1987 et Kinzie 1989). $[140,139]$

Le Propranolol (AVLOCARDYL®), Beta bloquant dont l'intérêt avait jusqu'alors surtout été montré en phase aigue (Pitman 2002, Vaiva 2003), permettrait de modérer la charge émotionnelle des souvenirs traumatiques lors de processus de remémoration active, même chez des patients présentant un ESPT chronique (Brunet 2008). [177, 214, 34]

\subsubsection{Un antituberculeux}

Un chercheur canadien spécialiste de l'ESPT, Stéphan Guay, base ses récentes recherches sur la D-Cyclosérine, antibiotique antituberculeux, qui utilisé en association à une psychothérapie, en favoriserait les effets thérapeutiques. Selon lui, «la D-Cyclosérine augmenterait la libération du glutamate, un neurotransmetteur qui favorise l'apprentissage et la mémorisation et atténue le sentiment de peur chez les personnes aux prises avec des troubles anxieux. » [12] 


\subsubsection{L'ocytocine}

Certains chercheurs optent pour la piste de l'ocytocine. Cette hormone produite naturellement par le cerveau est par exemple libérée lors de l'accouchement et à un rôle dans l'établissement du lien mère-bébé pendant les premiers mois. Olff et Frijling ont publié en juillet 2013 une revue de littérature dont certaines études évaluent cette molécule dans le traitement du stress et de l'ESPT. Ils partent de l'hypothèse que l'ocytocine cible l'amygdale et réduit la peur. Son administration à des patients, permettrait de réduire leur anxiété et ainsi faciliterait entre autres les interactions sociales. [168] 


\section{LES PUBLICATIONS EVALUANT LES DIFFERENTES THERAPEUTIQUES}

Il est nécessaire de compléter les références et recommandations citées précédemment par quelques publications majeures.

La publication référence des thérapies psychodynamiques dans le cadre de l'ESPT est celle de Brom et Kleber publiée en 1989. Elle a été menée chez 112 sujets souffrant d'ESPT sévère répartis en 4 groupes. 29 sujets bénéficiant d'une thérapie psychodynamique brève, 29 d'une hypnothérapie d'orientation behaviorale, 31 d'une désensibilisation et 23 formant le groupe contrôle. Les patients sont évalués avant la prise en charge puis à trois mois. Les résultats montrent une efficacité similaire pour les trois thérapies avec une diminution des symptômes cardinaux, contrairement au groupe contrôle. [31]

En 1998, Van Etten et Taylor publient leur méta-analyse de 39 études regroupant 61 essais évaluant plusieurs thérapeutiques, aussi bien les psychothérapies (thérapie par l'exposition, désensibilisation, relaxation, EMDR, hypnose, thérapie de soutien) que les traitements médicamenteux (entre autres Fluoxétine, Fluvoxamine, Phenelzine). Ils concluent que, pour réduire les symptômes d'ESPT, les psychothérapies sont plus efficaces que les traitements médicamenteux. Les psychothérapies efficientes sont les comportementales et l'EMDR, dont les effets sont maintenus à 15 semaines de suivi. Concernant les psychotropes, les meilleurs résultats semblent être avec les ISRS et les carbamazepines. La pharmacothérapie étant plus performante que les psychothérapies sur les symptômes de dépression. [218]

La méta-analyse de Bisson et Ehlers, publiée en 2007, regroupe 38 essais contrôlés randomisés, évaluant différentes thérapies dont la TFCBT (Trauma Focused CognitiveBehaviorial Therapy), l'EMDR, la thérapie de gestion du stress. Selon eux, la TFCBT et l'EMDR sont les thérapies de première ligne de l'ESPT. Elles ont une efficacité supérieure à la thérapie de gestion du stress ainsi que par rapport aux autres thérapies et n'ont pas de différence significative d'efficacité entre elles. [17] 
Il existe d'autres publications contradictoires :

Concernant l'EMDR, il y a celle de Davidson et Parker de 2001 [58]. Cette méta-analyse conclut que l'EMDR ne semble pas être plus efficace que les autres techniques d'exposition, de plus les mouvements oculaires, partie intégrante de la méthode ne sont pas nécessaires.

Bradley, Greene et al. publient en 2005 une méta-analyse incluant 26 études publiées entre 1980 et 2003 [24]. Elle évalue 44 traitements psychothérapeutiques dans le cadre de l'ESPT. Ils n'ont pas trouvé de différences significatives entre les différentes thérapies. La plupart des sujets après traitement continuaient d'avoir des symptômes résiduels. Ils mettent alors en avant les lacunes des études, dont le suivi trop court des patients inclus, en général de quelques mois mais qui devrait être d'au moins deux ans selon eux.

Deux méta-analyses (Sack 2001, Maxfield 2002) ont exclusivement cherché à analyser la méthodologie des études faites sur l'EMDR dans le traitement de l'ESPT, afin d'évaluer de manière précise les effets de cette thérapie. [185,156] 


\section{CONCLUSION DES THERAPEUTIQUES}

La thérapeutique de l'ESPT résulte donc de la juste association entre une prise en charge psychothérapeutique et un traitement médicamenteux, avec en première ligne les ISRS.

Nous aurions alors tendance à orienter la psychothérapie de l'ESPT vers les TCC, les thérapies d'exposition et l'EMDR, cependant, il est important de noter qu'elles sont plus étudiées et bénéficient donc de plus publications que les autres types de thérapies, notamment la psychodynamique.

De notre revue de publications, ressortent les limites méthodologiques des études évaluant les thérapies, ainsi que l'absence de véritable consensus clair sur la thérapie à employer. «Jusqu'à ce jour il n'y a pas de programme de traitement par excellence de l'ESPT et aucune approche thérapeutique particulière n'a encore reçu l'approbation universelle des cliniciens » (McFarlane et Yehuda, 2000). [157]

Nous n'avons abordé dans cet exposé que les thérapies individuelles, mais thérapies familiales, thérapies de groupes et autres groupes de paroles ne doivent pas être oubliés.

Le guide de la Haute Autorité de Santé, émis en Juin 2007, Affection de longue durée: trouble anxieux graves dans lequel sont exposées les recommandations de prise en charge de l'état de stress post-traumatique chronique, peut être l'une des références en France. On y trouve d'ailleurs la prise en compte incontournable des comorbidités nombreuses de 1'ESPT chronique mais aussi du soutien psychosocial. [116] 


\title{
QUATRIEME PARTIE
}

\author{
ETUDE PILOTE
}

\section{LES ADULTES AYANT ETE VICTIMES DE VIOLENCES SEXUELLES AVANT L'AGE DE 15 ANS : APPROCHE MEDICO-ECONOMIQUE}




\section{CONTEXTE DE L'ETUDE}

La motivation d'une étude sur l'impact médico-économique des violences sexuelles se trouve résumée dans le rapport de Mr André Ciavaldini, Les agressions sexuelles-Données Epidémiologique Générales : «L'ampleur des conséquences de ce phénomène de santé publique suppose pour cette dernière un coût élevé, d'autant plus que les troubles, lorsqu'ils se présentent sous une forme somatique, sont plus difficilement identifiables comme conséquences d'une agression sexuelle. Faute d'être repérée, et les victimes ne sont pas enclines à dévoiler ce qui est enfoui sous le poids de la honte, l'agression continuera son action muette fournissant sa charge de récurrence pathologique, augmentant encore le coût à moyen et long terme de soin. Il serait donc nécessaire de disposer d'études longitudinales permettant d'évaluer à moyen et long terme l'impact d'une agression sexuelle et donc ses divers pronostics. » $[43]$

En France, peu d'études se sont penchées sur cette question. Cependant, depuis peu, de par notre contexte économique et politique, rapports et publications semblent y porter un intérêt tout particulier. Les pays comme les Etats-Unis, l'Australie, le Canada et plus proche de nous l'Allemagne ont déjà plusieurs longueurs d'avance sur l'évaluation de l'impact économique des maltraitances et violences sexuelles.

Aux Etats-Unis, Suzanne Fromm Reed en 2001 retrouve des dépenses globales évaluées à 94 milliards. Dépenses globales en dollars, prenant en compte les coûts directs, besoins immédiats, mais aussi les indirects, visant les dépenses à long terme et celles liées aux effets secondaires. Les coûts directs (24 milliards) regroupent les hospitalisations (6 milliards), les problèmes chroniques de santé ( 2 milliards), le système de santé mentale (425 millions), la protection de l'enfance (14 milliards), la protection judiciaire (24 millions), le système judiciaire (341 millions). Les coûts indirects (69 milliards) comprennent l'éducation spécialisée (223 millions), la santé mentale et la protection de santé (4 milliards), la délinquance juvénile ( 8 milliards), la perte de productivité pour la société (656 millions) et la criminalité adulte (55 milliards). En 2007, Wang et Holtong, avec une étude similaire, retrouvent 33 milliards de dépenses directes, 71 milliards d'indirectes pour un coût global de 103 milliards de dollars. [104, 222] 
En Australie, Taylor, Moore Pelluzo et al. en 2008 avec une prévalence d'enfants de moins de 18 ans maltraités ou négligés, estimée entre 177000 et 666 000, le coût annuel économique et sociétal est selon eux de 4 milliards de dollars. Avec une incidence en 2007 entre 130237 et 490000 cas, ils projetaient un futur coût entre 13,7 et 38,7 milliards de dollars. [204]

Au Canada en 2003, McKenna, Bowlus et al. estiment le coût des mauvais traitements aux enfants en 1998 à 15705910047 dollars. [158]

Très récemment, une étude menée en Allemagne par Habetha, Bleich et al. (2012), retrouve un coût global sociétal, en lien avec les violences ou négligences psychiques, émotionnelles, physiques et sexuelles sur des enfants, entre 11,1 et 29,8 milliards d'euros avec une répercussion par allemand de 134,84 à 363,58 euros. [114]

D'autres publications recentrent leurs estimations sur les dépenses médicales. Ainsi, en 1997, Irazuzta, McJunkin et al., dans leur étude de cohorte rétrospective de patients admis dans USIPédiatrique en Virginie, entre janvier 1991 et août 1994, évaluent qu'une facture médicale pour les soins immédiats d'un enfant maltraité est en moyenne de 35641 dollars. [127]

En 1999, Walker, Unutzer et al. étudient les dépenses de santé chez des femmes. Ils retrouvent une majoration moyenne des dépenses de santé annuelles chez les femmes ayant été victime de maltraitances dans l'enfance de 95 dollars et de 245 dollars chez celles victimes de maltraitances sexuelles. [220]

Les résultats de la très grande étude menée par V.F. Felitti «Adverse Childhood Experiences » aux Etats-Unis, retrouve en 2008, une majoration du taux de prescriptions médicamenteuses de $40 \%$ entre des sujets n'ayant pas d'évènement traumatique dans l'enfance et ceux présentant un nombre d'évènements supérieur ou égal à cinq. [79]

Les chiffres de toutes ces publications vont dans le sens d'un surcoût par rapport à la population générale, qu'il semble alors nécessaire de quantifier aussi en France. 


\section{HYPOTHESES DE RECHERCHE ET OBJECTIFS}

A travers cette étude nous avons cherché à répondre aux questions suivantes :

- Les dépenses de santé chez des sujets adultes ayant été victimes de violences sexuelles dans leurs 15 premières années de vie, sont-elles supérieures à celles de la population générale, au cours des 12 derniers mois ? Si oui, de combien sont-elles ?

- Peut-on identifier des facteurs influençant ces dépenses?

Cette étude pilote peut permettre de tester la faisabilité de notre méthodologie et la validité de nos hypothèses, avant d'envisager une étude prospective de plus grande ampleur.

Si nous confirmons nos hypothèses, cela pourrait permettre d'identifier les dépenses de santé et d'appuyer la démarche de prévention, de renforcer les actions de dépistage et de traitement, le plus précocement possible.

\section{POPUlATION ET METHODE}

\subsection{POPULATION ETUDIEE}

Les sujets ont été recrutés au sein de la population de patients du service de psychiatrie et psychotraumatologie de l'Hôpital Tenon à Paris. Ils ont bénéficié d'une prise en charge psychiatrique dans le service que ce soit en hospitalisation ou en ambulatoire entre mai et octobre 2012.

Critères d'inclusion :

- Sujet ayant un diagnostic d'ESPT posé.

- Sujet ayant été victime de violences sexuelles au cours des 15 premières années de vie

- Sujet âgé d'au moins 18 ans, sans limite d'âge supérieure

- Sujet acceptant de participer à l'étude 
Critères de non inclusion :

- Sujet sous tutelle, curatelle ou incapable majeur

- Sujet n'ayant pas donné son consentement à l'étude

- Sujet ayant un diagnostic de psychose ou de démence posé

- Sujet dans l'incapacité de remplir les auto-questionnaires (illettré, analphabète)

\subsection{METHODOLOGIE}

\subsubsection{Modalité de recrutement et passation des questionnaires}

Au sein de la population définie précédemment, les patients sélectionnés étaient contactés par téléphone s'ils étaient en ambulatoire, ou directement abordés quand ils étaient en hospitalisation. Ils recevaient une information succincte, expliquant le contexte et l'objectif de l'étude mais aussi le déroulé de la passation. Ainsi, un accord oral était obtenu et un rendez vous ambulatoire fixé dans le service de psychiatrie à Tenon.

Le jour de la passation, le patient recevait de nouveau de manière orale, ainsi qu'à travers un document écrit qui lui était remis, des informations indispensables, notamment sur l'anonymat et le traitement des données.

La passation, en deux étapes, était d'une durée moyenne de 45 minutes. La première partie, plus fastidieuse, passée avec l'investigateur, collecte des données administratives ainsi que de consommation médicale. La seconde partie, plus simple, faite d'auto-évaluation avec les deux échelles internationales.

\subsubsection{Elaboration des questionnaires}

Deux types d'évaluations ont été utilisés. Un questionnaire d'hétéro-évaluation entièrement établi par l'investigateur pour répondre à la description de la population et surtout quantifier leurs dépenses de santé (document annexe 1). Une partie d'auto-questionnaire cherchant à évaluer brièvement la clinique du sujet (document annexe 2 et 3 ). 


\subsubsection{Collecte des données socio démographique et de santé globale}

Pour les données socio-démographiques, il s'agissait d'obtenir le lieu de naissance, l'âge, le sexe, la situation familiale et professionnelle et le niveau socio économique. Le niveau de santé globale actuel était évalué avec le poids et la taille, les pathologies déclarées par le sujet et ses consommations de toxiques. Pour les trois principaux toxiques (tabac, alcool, cannabis), nous avons recherché l'âge de première consommation, si la consommation était encore actuelle, ainsi que sa quantification. Pour le tabac le paquet-année a été utilisé. Pour l'alcool nous qualifions la consommation d'abusive lorsqu'elle était supérieure à 2 verres par jour pour les femmes et 3 pour les hommes. Pour le cannabis en estimant la consommation en nombre de joints par jour ou par semaine, nous pouvions savoir si la consommation du sujet était régulière ou occasionnelle. Pour les autres toxiques, cocaïne, crack, hallucinogènes, amphétamines, héroïne, etc., il s'agissait simplement de savoir s'il y avait déjà eu au moins une consommation au cours de la vie.

\subsubsection{Comment évaluer les dépenses de santé ?}

\section{Les outils d'élaboration du questionnaire}

L'évaluation des consommations a été basée sur les outils d'évaluation des dépenses de santé au niveau national. En effet, les comptes de la Santé en France comprennent différents agrégats :

\section{$\underline{\text { La Consommation Médicale Totale (CMT) }}$}

Comprend la Consommation de Soins et de Biens Médicaux (CSBM) et la Consommation des Services de Médecine Préventive (CSMP), (CMT=CSBM+CSMP)

- La Consommation de Soins et de Biens Médicaux :

Correspond à l'ensemble des biens et services achetés par les ménages et comprend :

- Les soins hospitaliers : publics et privés

- Les soins ambulatoires : médecin, auxiliaire médical, dentiste, analyse, cure thermale

- Transport de malades

- Médicaments

- Autres biens médicaux : optique, prothèses, orthèses, véhicules pour handicapés physiques, petit matériel. 
- La Consommation de Services de Médecine Préventive :

Correspond entre autres à la médecine du travail, la médecine scolaire, la protection maternelle et infantile.

\section{Les Dépenses Courantes de Santé}

Couvrent un champ plus large que la CMT, elles comprennent également les indemnités journalières, la prévention collective, les subventions reçues par le système de santé, le fonctionnement de la recherche, l'enseignement et de l'administration sanitaire.

\section{Les Dépenses Pour les Malades}

Comprennent la CSBM, les indemnités journalières et les soins de longue durée (services de soins à domicile, soins aux personnes en établissement)

Le questionnaire a été établi pour pouvoir calculer pour chaque sujet, de manière rétrospective sur l'année écoulée, la consommation médicale totale. En effet, les dépenses courantes de santé n'ont pas été recherchées, car trop complexes à évaluer à l'échelle individuelle. Les enveloppes qu'elles regroupent, par exemple, les subventions et budget attribués à la recherche, l'enseignement et l'administration sanitaire ne peuvent être obtenues avec notre questionnaire.

\section{Modalités d'investigation des dépenses}

Afin d'évaluer la consommation de médicaments du sujet, il fallait obtenir, si possible avec l'aide de ses ordonnances, le nom des produits (distinguant générique ou princeps) et ses caractéristiques (dosage et forme galénique), la quantité consommée par jour et le nombre de fois par semaine. Pour chaque molécule citée, nous lui demandions s'il s'agissait d'automédication ou de prescription par un médecin.

Pour les soins ambulatoires, il s'agissait de demander au sujet le nombre de consultations au cours des douze derniers mois, chez un généraliste et chez des spécialistes. S'il s'en souvenait il nous donnait à titre indicatif les tarifs pratiqués. Il pouvait aussi nous donner l'identité du médecin et le lieu de consultation afin que nous puissions retrouver dans un second temps les tarifs appliqués, sur les sites de la sécurité sociale. Il lui était demandé de se rappeler des examens complémentaires prescrits et réalisés, aussi bien les examens biologiques que tous types d'imagerie. 
Le nombre de recours dans l'année à un service d'urgences était demandé ainsi que les examens pratiqués.

Le nombre d'hospitalisations mais aussi les actes chirurgicaux ont été évalués comprenant alors le type de service, la durée et le lieu de l'hospitalisation.

Les prises en charge paramédicales étaient passées en revue (psychologue, dentiste, kinésithérapeute, ostéopathe, orthophoniste, hôpital de jour etc.) et les tarifs estimés. Pour les chirurgiens dentistes, les actes pratiqués étaient demandés. Les prises en charges paramédicales à domicile étaient aussi recherchées.

Il était de plus demandé au sujet s'il avait bénéficié d'une cure thermale dans l'année, mais aussi des services d'un opticien ou s'il avait d'autres biens médicaux tels que des prothèses auditives, orthèses ou attelles.

Nous demandions au sujet le nombre de recours aux services du SAMU, des pompiers ou encore d'SOS médecin au cours des 12 mois précédents. L'horaire d'intervention devait être précisé.

Concernant le transport de malade, il s'agissait de retrouver le type de transport (taxi conventionné, véhicule sanitaire léger ou ambulance), le trajet effectué et le créneau horaire.

Le recours à un service de médecine préventive était recherché.

Nous demandions au sujet s'il avait bénéficié au cours des douze derniers mois d'un ou de plusieurs arrêts de travail et leur durée.

Deux questions supplémentaires permettaient au sujet de compléter le questionnaire précédent. Il pouvait nous citer des dépenses qu'il aurait omis de nous donner ou encore s'il estimait que nous avions oublié de lui en demander. 


\subsubsection{Evènements de vie}

La dernière partie de l'hétéro questionnaire avait pour but de définir le ou les évènements traumatiques. Confirmer les violences sexuelles avant l'âge de 15 ans en distinguant les cas de viol ou d'autres violences sexuelles, mais aussi obtenir des informations sur l'agresseur, comme son sexe, son âge approximatif au moment des faits ou le lien avec la victime (connaissance, membre de la famille, voisin, ami...)

Deux autres informations ont été recherchées, le délai entre les faits et la prise en charge psychiatrique ou psychologique ainsi que l'existence d'autres évènements traumatiques à l'âge adulte.

\subsubsection{Evaluation clinique grâce à deux auto-questionnaires}

La partie d'auto évaluation permettant une évaluation clinique succincte comprend deux échelles :

- L'échelle de Beck aussi appelée Beck Depression Inventory (BDI), recherche une éventuelle symptomatologie dépressive. Elle comprend 21 items, avec 4 possibilités graduelles de réponses cotées de 0 à 3 . Le sujet entoure pour chaque item la réponse qui lui correspond le mieux. Ainsi, un total est obtenu sur 63. (Document annexe 2)

- L'IES-R (Impact of Event Scale-Revised) évalue l'impact de l'évènement traumatique en ciblant les symptômes neurovégétatifs tels que les manifestations d'intrusion, d'hypervigilance et d'évitement. Composée de 22 items sous forme d'affirmation, le patient doit noter si celle-ci lui correspond, «pas du tout » à « extrêmement » (0 à 4). On peut alors calculer un score sur 88. Cette échelle est l'une des plus utilisée pour les essais cliniques et a été validée dans ce cadre par le comité d'experts de l'European College of Neuropsychopharmacology (Document annexe 3). 


\subsubsection{Traitement des informations recueillies grâce aux questionnaires}

Les informations socio-démographiques ainsi que les données concernant l'évènement n'ont pas nécessité de traitement particulier.

L'Indice de Masse Corporel était calculé selon la formule consacrée poids/taille ${ }^{2}$ et ainsi, le sujet était catégorisé avec la valeur de son IMC selon les valeurs internationales.

Les pathologies intercurrentes déclarées ont été codées à l'aide de la CIM-10.

Les coûts ont été estimés selon les dires des patients, confirmés grâce aux investigations directes auprès des structures telles que les cliniques privées, mais surtout avec l'aide des sites internet de la sécurité sociale ameli.fr et ameli-direct.fr.

Certains calculs doivent être expliqués. Ainsi, pour les traitements médicamenteux, une estimation du coût sur la semaine pouvait être faite grâce aux Prix Publics Imposés obtenus sur le site Eurekasanté.fr par Vidal et ainsi qu'une extrapolation grossière sur l'année. Pour la biologie réalisée en ambulatoire, la nomenclature standard a été appliquée. Il s'agit d'appliquer le coefficient $\mathrm{B}$ à la valeur de l'examen. B ayant pour valeur 0,27 lorsque l'examen est pratiqué en laboratoire et en journée. Par exemple, la valeur d'une glycémie à jeun dans le contexte cité précédemment est 5 B donc 5X0,27=1,35 euros. Pour le recours aux urgences, il faut savoir que si les examens complémentaires pratiqués dépassent 91 euros, ils sont comptabilisés en supplément du coût forfaitaire d'une prise en charge aux urgences. Pour le transport de malade, le coût était calculé à partir du type de transport, de l'horaire et du kilométrage approximatif en appliquant les tarifs donnés par la sécurité sociale. Les dépenses en lunetterie ont été retrouvées grâce aux factures des patients ou alors calculées approximativement avec les corrections des patients avec l'aide d'un opticien appliquant les tarifs de la sécurité sociale y compris le forfait CMU. Pour les orthèses et attelles, les tarifs moyens sont ceux fixés par la LPPR (Liste des Prestations et Produits Remboursables) définie par l'assurance maladie.

Les valeurs chiffrées des dépenses ainsi obtenues étaient regroupées et sommées afin d'obtenir les différents agrégats cités précédemment pour chaque sujet. 
Le score de l'échelle de Beck, obtenu sur 63, permet d'alerter sur une possibilité de syndrome dépressif actuel et de qualifier le degré de dépression : moins de 11 points, pas de dépression ; de 12 à 19 points, dépression légère; de 20 à 26 points, dépression modérée ; plus de 26 points, dépression majeure ou grave.

Le score de l'IES-R quant à lui donne une indication sur l'éventualité d'un ESPT : inférieur à 11 il y a peu de signe, entre 12 et 32 le patient présente bon nombre de symptômes, supérieur à 33 le patient présente probablement un état de stress post traumatique.

\subsubsection{Analyse statistique}

Dans un premier temps, nous allons simplement aborder les résultats de manière descriptive, permettant ainsi de décrire notre population et ses dépenses. Dans un second temps, grâce à des outils statistiques nous allons tenter de vérifier notre hypothèse principale ainsi que les secondaires.

Nous utiliserons pour la comparaison d'une moyenne théorique et d'une moyenne observée le test $t$ de Student avec comme condition d'applicabilité que la moyenne théorique soit répartie selon une loi normale.

Puis compte tenu de notre petit échantillon pour rechercher une corrélation entre deux variables, nous allons utiliser le test corrélation de Spearman.

Pour comparer les moyennes de deux groupes, nous utiliserons le test de Mann-Whitney.

Du fait de la répétition des tests statistiques, avec un intervalle de confiance à $95 \%$, la significativité des résultats sera avec un $\mathrm{p}<0,01$. 


\section{RESULTATS}

\subsection{STATISTIQUES DESCRIPTIVES}

\subsubsection{Caractéristiques de la population de l'étude}

17 sujets pouvaient être sélectionnés. Seules deux patientes contactées n'ont pas voulu donner suite. 15 patients ont donc été recrutés, parmi eux 14 femmes et 1 homme entre 25 et 60 ans (moyenne d'âge 41,26 ans).

1 seul sujet est né à l'étranger (en Inde), les 14 autres sont nés en France. 13 sujets résident actuellement en Ile-de-France, 1 habite la Picardie, 1 autre le Languedoc-Roussillon.

Concernant leur statut matrimonial (Figure 1), aucun n'est marié, 11 sont célibataires, 3 sont pacsés ou en concubinage et 1 est divorcé. 11 sujets sont sans enfant, 4 n'ont qu'un seul enfant. On observe alors 3 sujets célibataires avec enfants.

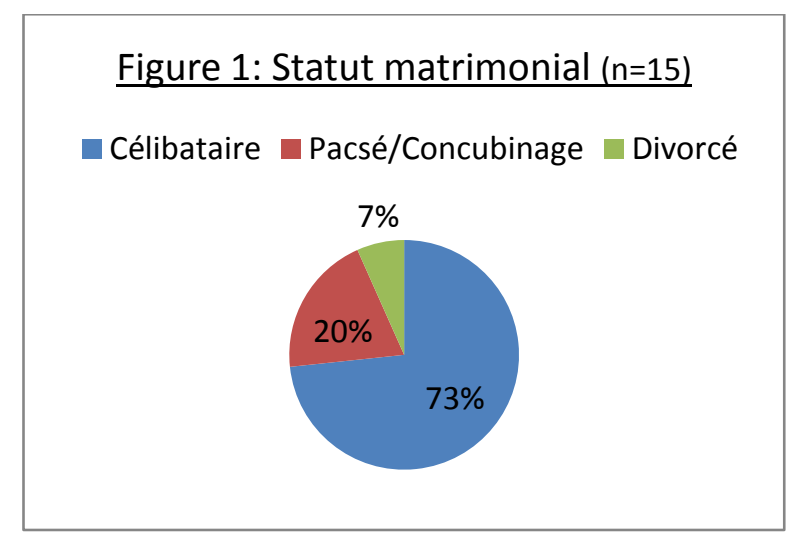

Pour le niveau socio économique, 12 sujets sont locataires de leur logement, 2 sont propriétaires et 1 sujet (le plus jeune de notre échantillon) vit chez ses parents (Figure 2). 9 sujets sont au chômage et 6 sont actifs aucun n'est donc étudiant ou retraité (Figure 3 ).

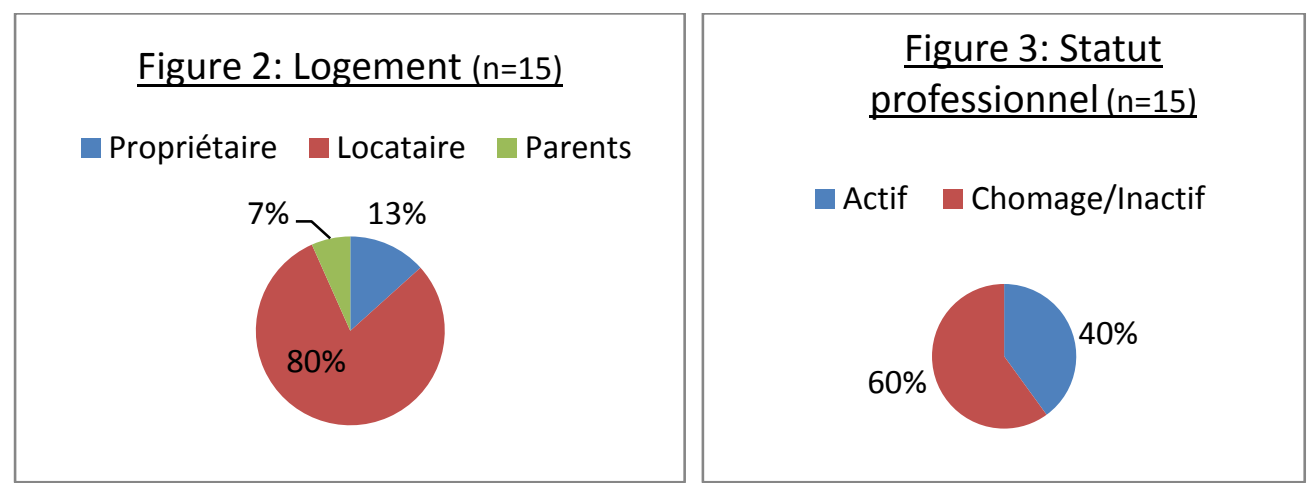


Parmi les actifs, on retrouve 2 employés de la fonction publique, 2 exerçant une profession intermédiaire (de l'enseignement, de la santé, de la fonction publique ou assimilé), 1 cadre de la fonction publique et 1 employé administratif d'entreprise. Ainsi, 5 sujets sur les 6 actifs travaillent dans la fonction publique. Parmi les chômeurs et inactifs, les niveaux d'étude selon la classification de l'Education Nationale et du ministère chargé de l'Enseignement supérieur sont répartis de la manière suivante : 3 sujets niveau I et II (Licence, Maitrise, Doctorat ou diplôme de grandes écoles...), 2 niveau III (Diplôme d'état, Brevet de technicien supérieur, Diplôme universitaire de technologie...), 2 niveau V (Certificat d'aptitude professionnel, Brevet d'étude professionnel) et 2 niveau $\mathrm{V}$ bis (Certificat de formation, Diplôme national de brevet).

Concernant les prestations sociales, 9 sujets bénéficient de 1'Affection Longue Durée, 3 de la Couverture Maladie Universelle. 11 sujets cotisent pour une mutuelle.

Les autres prestations dont bénéficie notre population se repartissent selon la figure 4.

\section{Figure 4 : Nombre de sujets par type de prestation sociale}

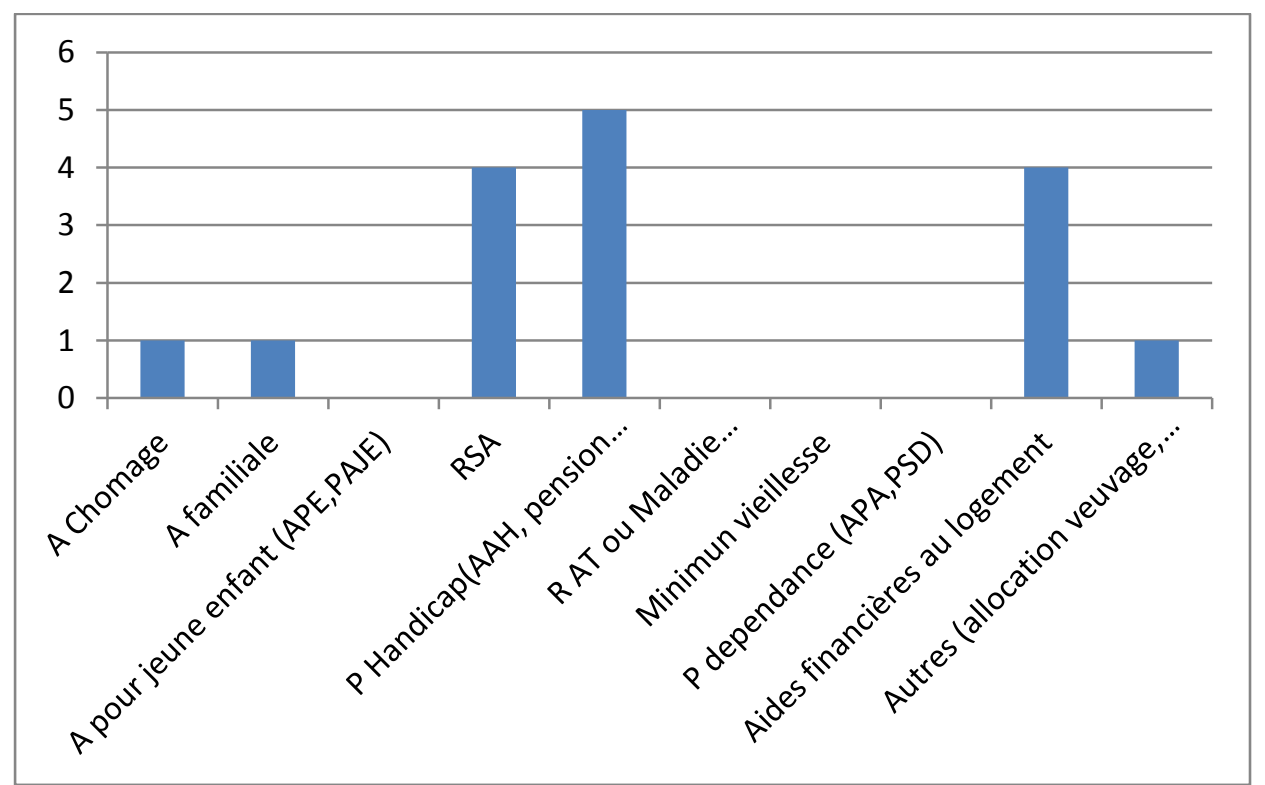

$\mathrm{A}=$ Allocations ; $\mathrm{P}=$ Prestation, $\mathrm{RAT}=$ Rente d'accident du travail, $\mathrm{RSA}=$ Revenu de solidarité actif

Nous avons une estimation des revenus nets mensuels des sujets inclus. Ainsi, le revenu mensuel moyen des sujets inclus est de 1371,73 euros avec un maximum de 2700 euros et un minimum de 417 euros. 


\subsubsection{La santé globale de notre échantillon}

Le calcul de l'Indice de Masse Corporel (IMC) a permis d'obtenir la répartition suivante (Figure 5)

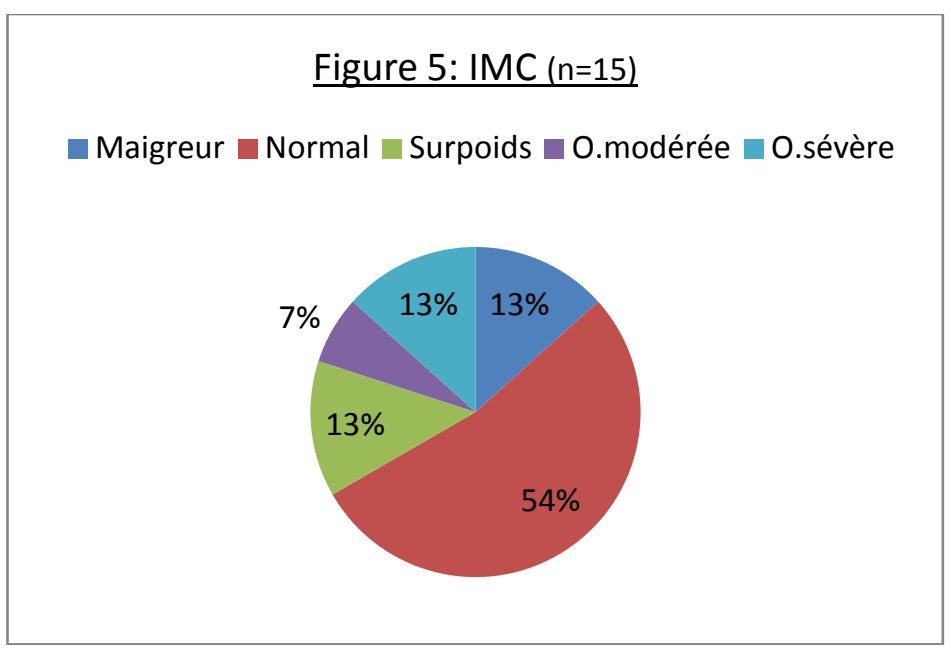

7 sujets présentent un trouble du poids : 2 sont en surpoids, 3 sont obèses et 2 sont maigres

8 sujets ont un poids normal

Les pathologies déclarées ont été classifiées pour plus de facilité selon la CIM 10.

On retrouve alors :

- 13 sujets sur 15 reconnaissent un trouble psychiatrique

- 4 sujets déclarent une ou plusieurs pathologies endocriniennes

- 1 sujet déclare une pathologie du système nerveux

- 2 sujets ont une pathologie ORL

- 2 sujets ont une ou plusieurs pathologies circulatoires (entre autre hypertension artérielle)

- 4 sujets ont une pathologie du système digestif

- 5 sujets déclarent une pathologie ostéo-articulaire, musculaire ou du tissu conjonctif.

- 2 sujets ont une pathologie de l'appareil génito-urinaire

La figure 6 montre la répartition des sujets en fonction du nombre de pathologies qu'ils ont déclarées.

Nous constatons alors que 2 sujets déclarent n'avoir aucune pathologie pas même d'ordre psychiatrique. 
Figure 6: Nombre de pathologies déclarées par nombre de sujet

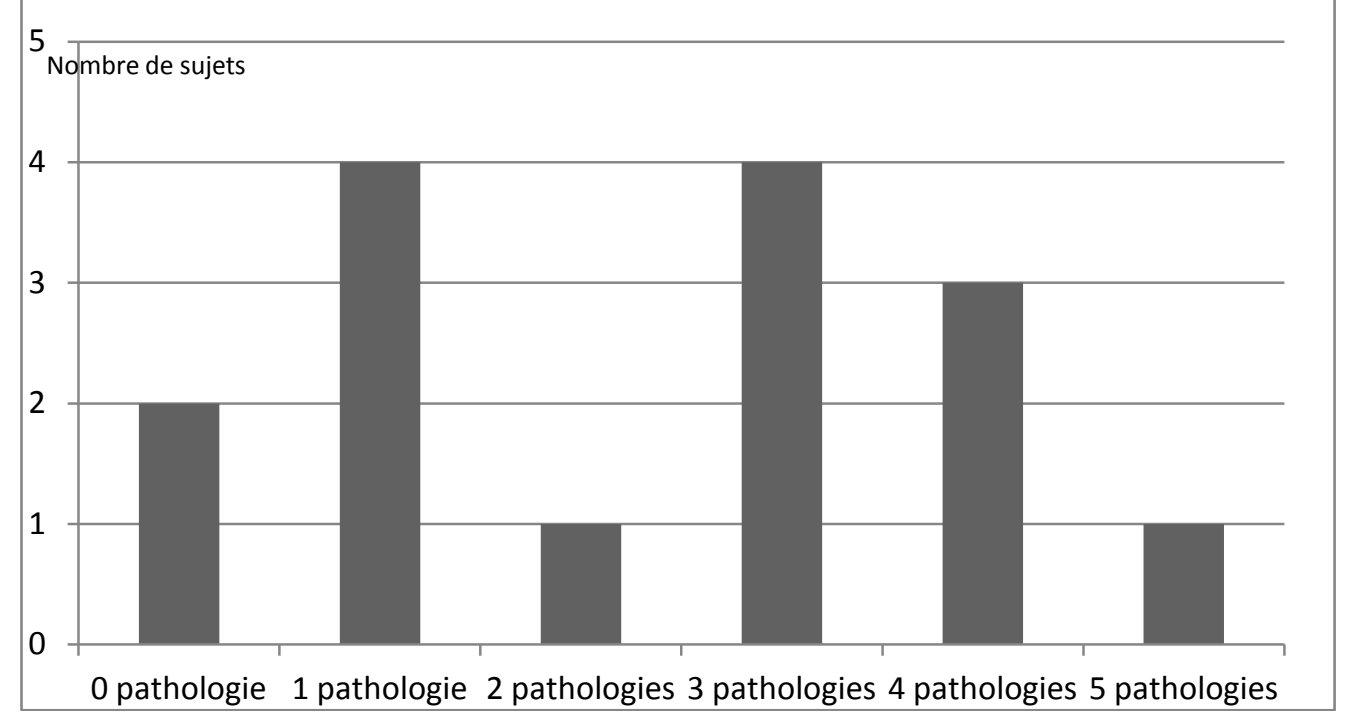

La consommation de toxiques

Consommation de tabac :

12 sujets ont déjà consommé du tabac au cours de leur vie.

L'âge moyen de la première cigarette est de 14,25 ans (minimum 6 ans, maximum 20 ans). Notre population est dans la fourchette nationale.

9 sujets ont actuellement un tabagisme actif avec une consommation moyenne de 26,3 Paquet-Année (minimum 8,75 PA et maximum 92 PA).

Consommation d'alcool :

14 sujets ont déjà consommé de l'alcool.

L'âge moyen de la première consommation est 17,4 ans (Minimum 10 ans, Maximum 30 ans). Actuellement 4 sujets ont une consommation abusive d'alcool.

\section{Consommation de cannabis :}

8 sujets ont déjà consommé du cannabis.

L'âge moyen de consommation est 12,2 ans (minimum 16 ans et maximum 35 ans). Actuellement, 8 sujets consomment régulièrement du cannabis dont 2 sont des consommateurs quotidiens. 


\section{$\underline{\text { Autres toxiques : }}$}

Les sédatifs et hypnotiques en dehors des doses prescrites sont les autres toxiques les plus utilisés. Au même niveau, nous retrouvons la cocaïne, le crack, les stimulants et les hallucinogènes.

La figure 7 montre la répartition des toxiques autres que tabac, alcool, cannabis expérimentés dans notre échantillon.

Figure 7 : Nombre de sujet par type de toxique expérimenté

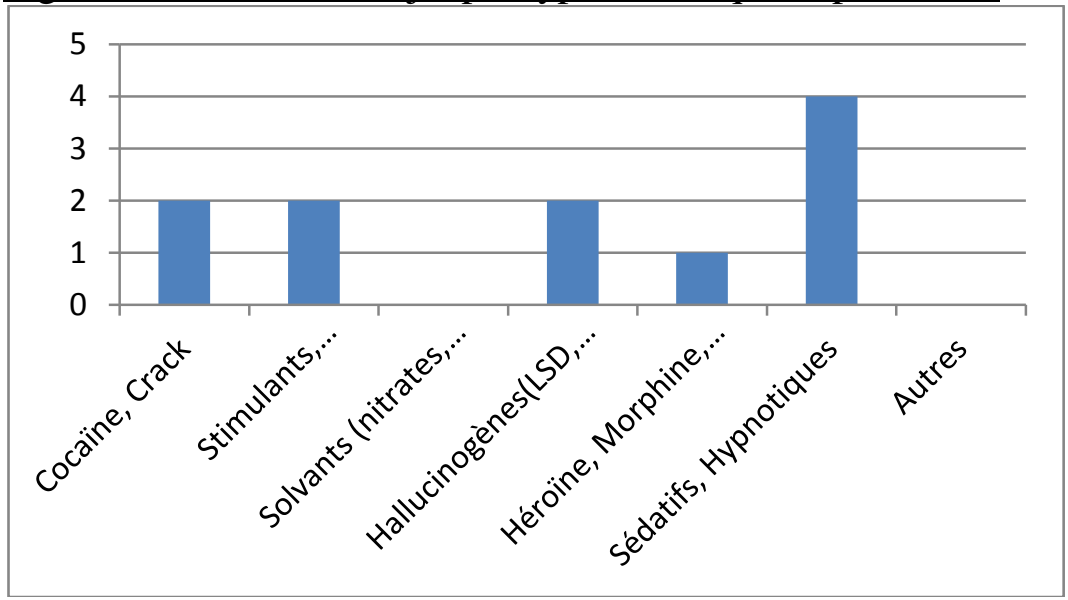

\subsubsection{Les évènements de vie}

13 sujets ont été victimes de viol et 2 d'autres types de violences sexuelles.

L'âge moyen au moment des faits est de 7,7 ans, le plus jeune avait 5 ans et le plus âgé avait 14 ans.

L'agresseur avait un âge approximatif maximum de 50 ans et minimum de 11 ans. Ce qui fait une moyenne d'âge de 28 ans. 6 agresseurs étaient des mineurs et donc 9 étaient des individus majeurs. Dans 14 cas, il était de sexe masculin. 3 sujets ont rapporté avoir été victimes de plusieurs agresseurs. Pour 14 victimes interrogées sur 15, l'agresseur était une connaissance, et pour 11 sujets, il s'agissait d'un membre de la famille. Le lien à l'agresseur est détaillé figure 8 . 
Figure 8: Nombre de sujet par lien avec l'agresseur

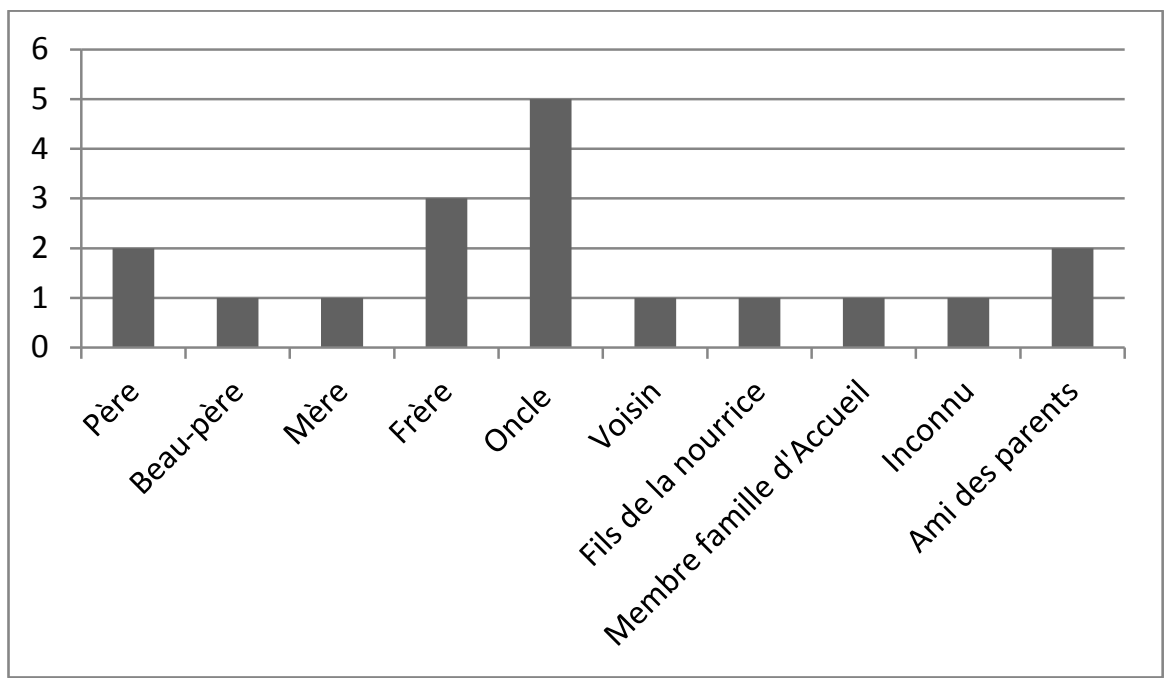

12 sujets considèrent avoir vécu au moins un autre évènement traumatique à l'âge adulte.

L'âge moyen lors de la première prise en charge psychiatrique ou psychologique est de 22,7 ans avec l'âge maximum retrouvé à 40 ans et minimum à 10 ans. Nous obtenons alors un délai de prise en charge moyen de 15 ans après les faits, le maximum de 34 ans et le minimum de 0 année.

\subsubsection{Les échelles d'auto évaluation}

\section{Le Beck Depression Inventory}

Le score maximal est de 42 , le minimal de 3 . On obtient un score moyen pour la population de 22,4 .

On peut observer la répartition au sein de la population grâce à la figure 9 :

Nous constatons possiblement une absence de dépression chez 3 sujets (score $<11$ ), une dépression légère chez 2 patients (entre 12 et 19), une dépression modérée chez 5 patients (entre 20 et 26) et une probable dépression majeure chez 5 sujets (score > 26). 


\section{Figure 9: Score de BDI au sein de l'échantillon}

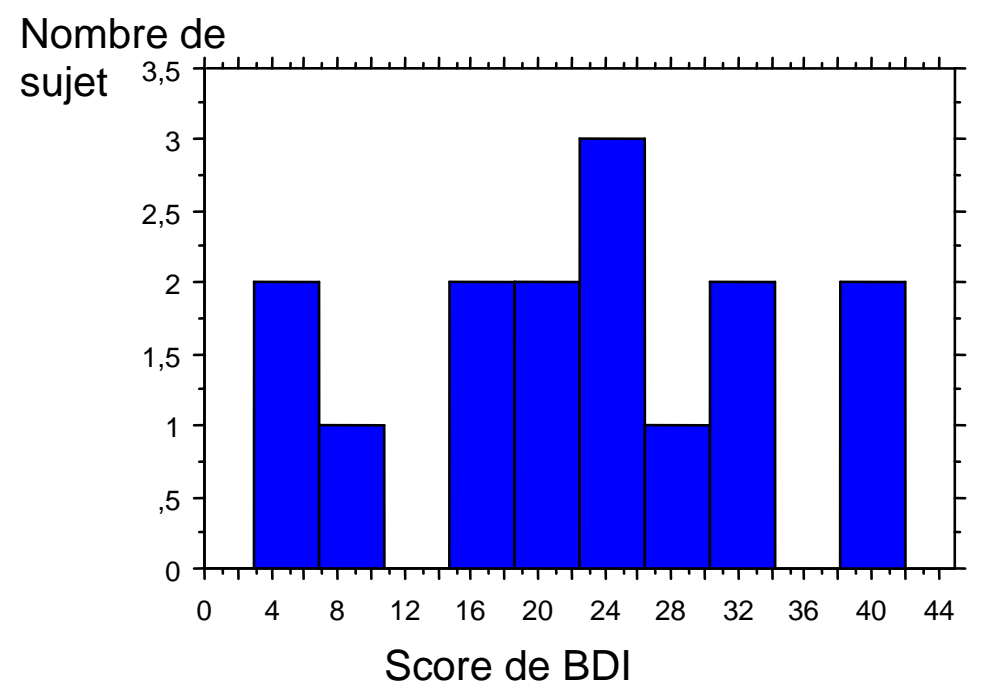

L'IES-R ou Impact of Event Scale-Revised

Le score moyen dans la population étudiée est de 49,73 avec un maximum à 78 et un minimum à 4 .

La répartition au sein de notre population se trouve sur la figure 10 :

1 sujet semble n'avoir que peu de signe d'ESPT et 10 présentent très probablement un ESPT.

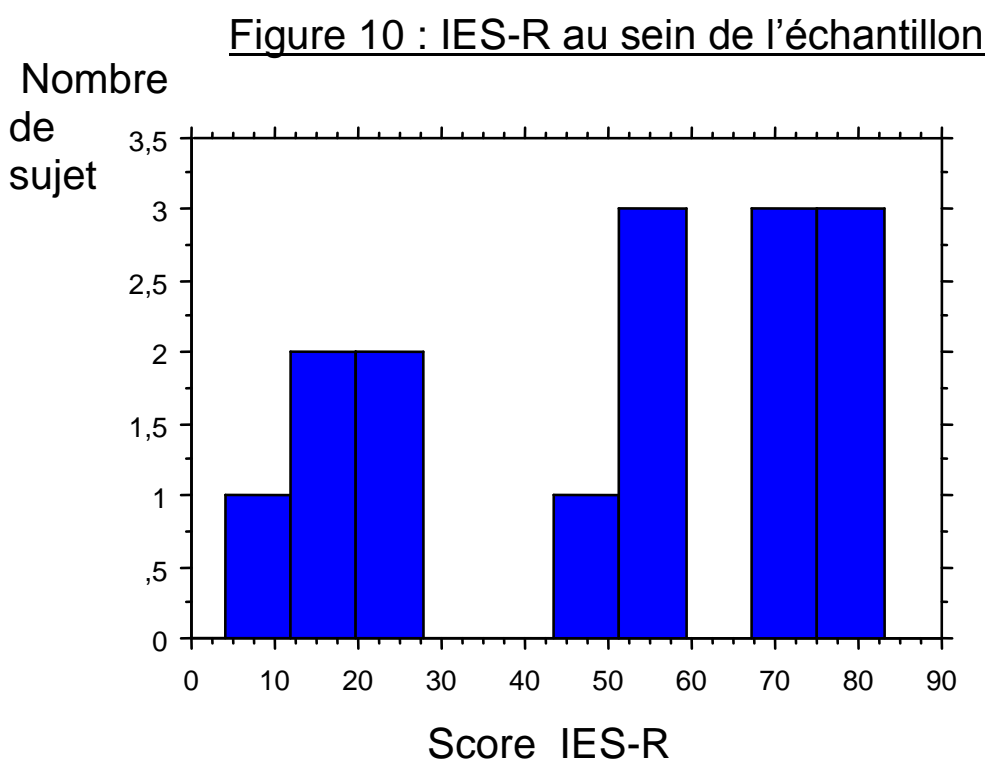




\subsubsection{Les dépenses de santé de notre population}

\subsubsection{Calcul des dépenses et données associées obtenues}

\section{Les traitements médicamenteux sur une semaine :}

- 2 sujets n'ont bénéficié d'aucun traitement médicamenteux.

- Le montant total sur 7 jours par sujet : maximum $=148,88 €$ et moyenne $=35,63 €$

- Le montant des psychotropes par sujet : maximum $=139,72 €$ et moyenne $=5,81 €$

- Le montant des antalgiques par sujet : maximum $=69,09 €$ et moyenne $=6,26 €$

-Dans les dépenses liées aux traitements médicamenteux le pourcentage moyen de psychotropes est de 35,04\% (maximum 100\%, minimum 0\%), d'antalgiques 13,82\% et d'automédication $18,92 \%$.

\section{Soins ambulatoires et hospitaliers au cours des 12 derniers mois :}

- Les consultations chez le généraliste: 3 sujets n'ont eu aucune consultation chez leur généraliste au cours de l'année. Le nombre de consultations maximum pour un même patient est de 15 consultations. La moyenne est de 6,26 consultations par sujet.

- Les consultations aux urgences : maximum 1 fois et ce pour 5 sujets.

- Aucun sujet n'a fait appel à un service tel que SOS médecin.

- Les hospitalisations : 7 sujets n'ont eu aucune hospitalisation dans l'année. Le maximum étant 4 hospitalisations pour un même patient.

- Les hospitalisations en psychiatrie : 5 sujets ont été hospitalisés en psychiatrie dans l'année. Les hospitalisations les plus longues, 230 jours et 225 jours, sont celles de deux sujets ayant bénéficié de 2 hospitalisations dans l'année. La durée moyenne d'hospitalisation en psychiatrie de 37,13 jours dans notre échantillon. 5 sujets ont été hospitalisés dans des services non psychiatriques et non chirurgicaux. 1 sujet a été hospitalisé dans un service de chirurgie. 
- Les consultations chez des spécialistes au cours de l'année : le maximum de consultations spécialisées est de 34, pour une moyenne de 10,13 consultations dans notre population au cours des 12 derniers mois. 1 sujet n'a eu aucune consultation de spécialiste dans l'année.

- Les consultations chez un psychiatre : le nombre maximum est de 32 consultations pour un sujet et une moyenne de 5,4 consultations dans notre population. 4 sujets n'ont pas eu de consultation avec un psychiatre dans l'année.

- Un maximum de 20 consultations et une moyenne de 4,5 sont retrouvés pour les consultations spécialisées hors psychiatrie. Les spécialistes après les psychiatres les plus consultés sont les gynécologues (la population recrutée est largement féminine), les ophtalmologues puis les dermatologues.

La figure 11 permet d'observer la répartition des consultations de spécialistes au sein de notre population.

Figure 11: Pourcentage de sujet ayant consulté au moins une fois par type de spécialiste

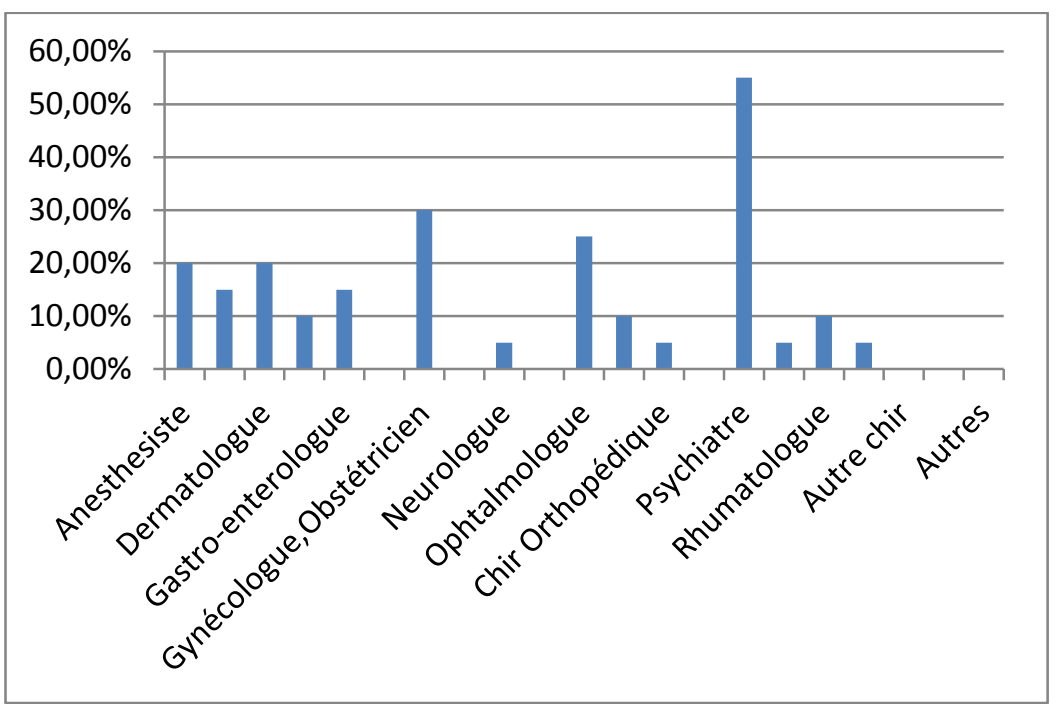

\section{Les consultations de paramédicaux :}

Tous les sujets interrogés ont consultés des paramédicaux. La dépense maximale sur l'année écoulée est de $6636 €$, le minimum est de $20 €$ pour une moyenne de $1964,61 €$.

La part liée aux soins en santé mentale est de 1 054,66 € en moyenne avec un maximum de $6636 €$ et un minimum de $0 €$. 


\section{Les paramédicaux à domicile :}

Aucune prise en charge paramédicale à domicile n'a été retrouvée.

\section{Cure Thermale :}

Aucun sujet n'a été en cure thermale

\section{La consommation de biens médicaux :}

- 4 sujets ont bénéficié des services d'un opticien dans l'année. La facture maximale étant de 1811 euros et la moyenne de 277,70 euros.

- 4 sujets sont concernés par l'achat de prothèse, d'orthèse ou d'attelle. On évalue alors la dépense maximale sur l'année à 3950 euros et une moyenne de 292,67 euros.

\section{Transport de malade au cours de 12 derniers mois :}

- Aucun n'a eu recours au SAMU.

- 3 sujets ont bénéficié de jour de transport par VSL (Véhicule Sanitaire Léger), ambulance ou taxi conventionné. Le coût maximal est de 730,71 euros sur l'année et la moyenne est de 56,27 euros.

\section{Médecine préventive :}

5 sujets ont eu recours à la médecine du travail sur 6 actifs. Ces 79,53 euros annuels par salarié sont versés par l'employeur.

\section{Dépenses complémentaires :}

- Un sujet a fait appel aux pompiers.

- 6 sujets ont bénéficié d'un arrêt de travail de plus de 3 jours. La moyenne d'arrêt étant de 57,4 jours dans l'année avec un coût moyen de 3 286,82 euros. 


\subsubsection{Observation de la consommation annuelle de notre population}

Toutes les données que nous avons pu rassembler vont être utilisées pour calculer les différents postes de dépenses en prenant pour modèle les agrégats nationaux. Le tableau 5 synthétise le calcul des dépenses annuelles pour les différents agrégats au sein de notre population.

$\underline{\text { Tableau } 5 \text { : Dépenses totales annuelles en euros pour chaque agrégat }}$

\begin{tabular}{|c|c|c|c|}
\hline & Coût minimum & Coût maximum & Coût Moyen \\
\hline $\begin{array}{c}\text { Consommation de soins } \\
\text { hospitaliers }\end{array}$ & 0 & 101685,95 & 23972,13 \\
\hline $\begin{array}{c}\text { Consommation de soins } \\
\text { ambulatoires }\end{array}$ & 197,10 & 11141,92 & 3057,52 \\
\hline Transport de malade & 0 & 730,71 & 113,45 \\
\hline $\begin{array}{l}\text { Consommation de } \\
\text { médicaments }\end{array}$ & 0 & 7741,76 & 1854,68 \\
\hline $\begin{array}{l}\text { Consommation d'autres } \\
\text { biens médicaux }\end{array}$ & 0 & 3950 & 571,37 \\
\hline $\begin{array}{l}\text { Consommation de soins } \\
\text { et de biens médicaux }\end{array}$ & 683,20 & 117574,92 & 29569,15 \\
\hline $\begin{array}{l}\text { Consommation } \\
\text { médicale totale }\end{array}$ & 683,20 & 117574,92 & 29605,35 \\
\hline $\begin{array}{l}\text { Dépenses globales } \\
\text { annuelles évaluées }\end{array}$ & 683,20 & 124774,92 & 32892,17 \\
\hline
\end{tabular}

Nous avons nommés «dépenses globales », toutes les dépenses investiguées y compris les interventions des pompiers ou les indemnités journalières. Cependant, pour le reste de l'étude nous nous en tiendrons à la Consommation Médicale Totale (CMT). 
La figure 12 schématise les moyennes des différents agrégats avec leur écart type. On voit ainsi que la Consommation de Soins Hospitaliers est d'une grande variabilité entre les sujets.

\section{Figure 12 : Moyennes annuelles des différents postes de dépenses de santé (écart type)}

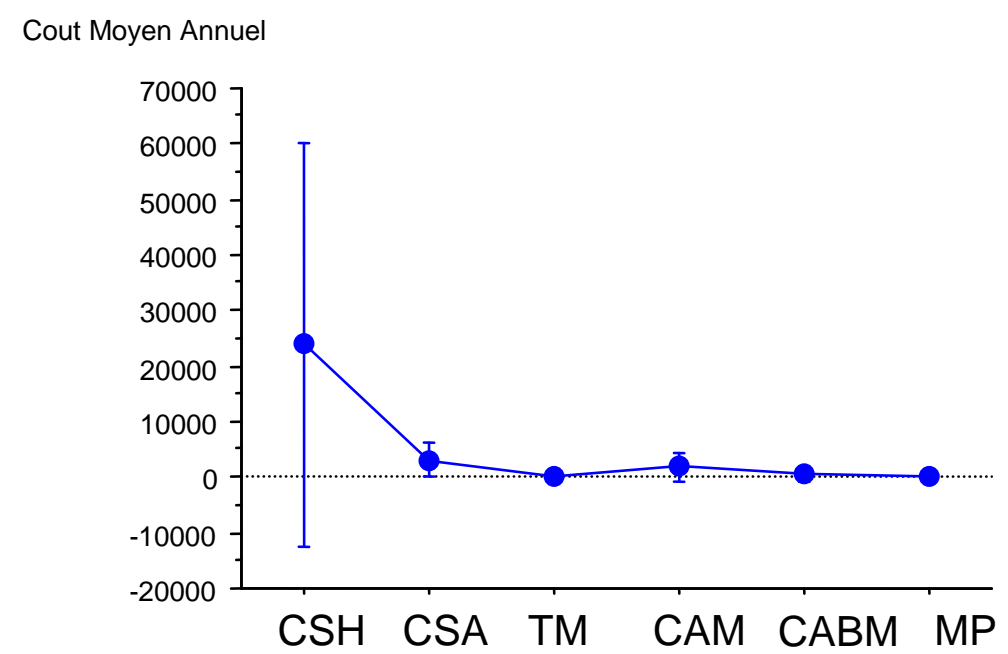

CSH : Consommation de soins hospitaliers, CSA : Consommation de soins ambulatoires TM : Transport de malade, CAM : Consommation annuelle de médicaments CABM : Consommation d'autres biens médicaux, MP : Médecine préventive

La figure 13 permet d'apprécier la répartition de la consommation médicale totale dans la population étudiée. La majorité des sujets ont des dépenses inférieures à 20000 euros par an. Seuls deux patients possèdent des dépenses « extrêmes », supérieures à 100000 euros.

\section{Figure 13: Consommation Médicale Totale au sein de l'échantillon}

Nombre de

sujet

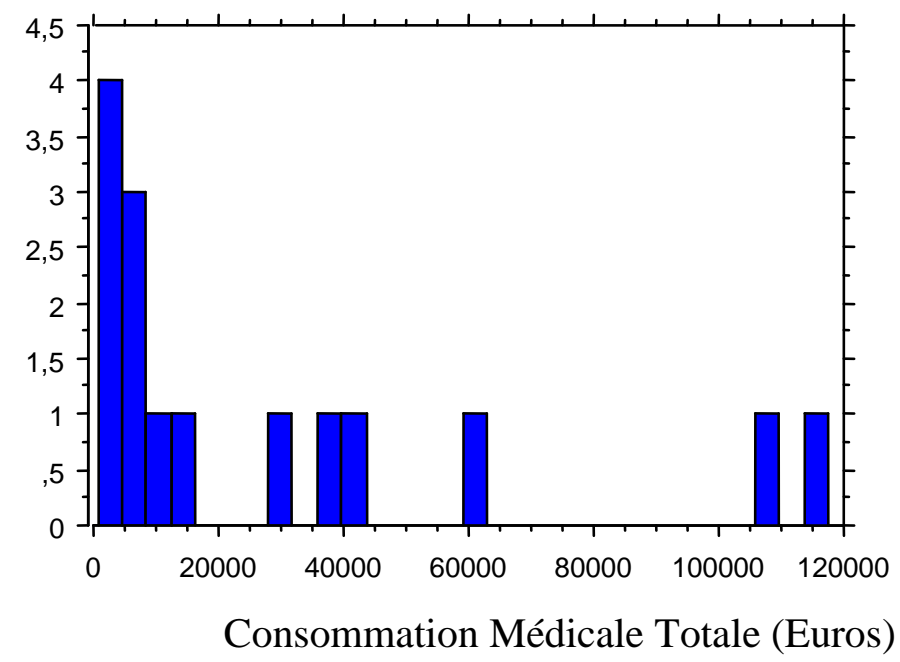


Les dépenses de notre échantillon (Figure 14) se répartissent par ordre décroissant de la manière suivante: les hospitalisations, le paramédical, les médicaments, les médecins, les autres biens médicaux et enfin transport et médecine préventive.

Figure 14 : Répartition des dépenses dans notre échantillon

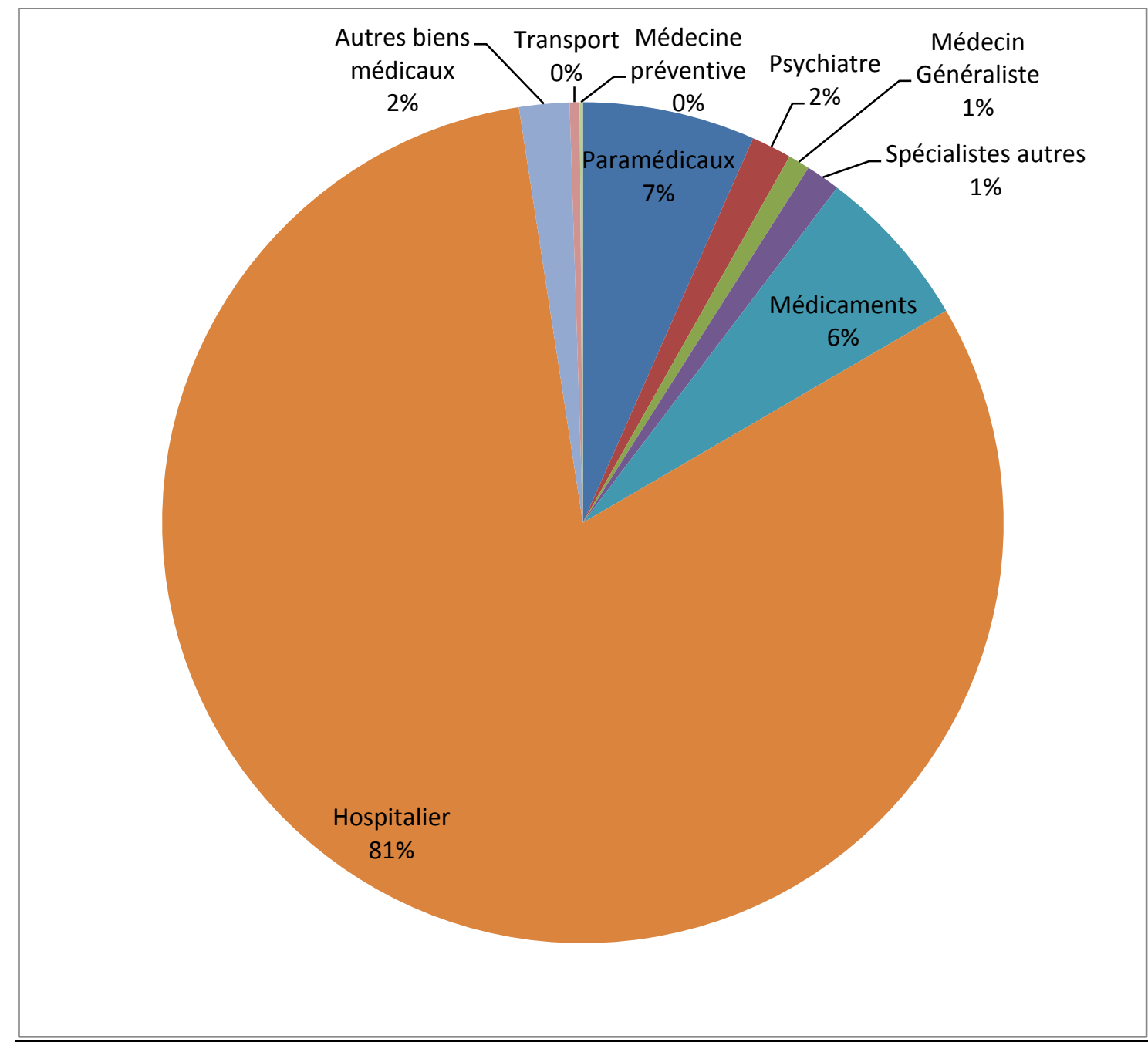




\subsection{EVALUATION STATISTIQUE}

\subsubsection{Quelques éléments descriptifs de la population française.}

En 2012 le Salaire Minimum Interprofessionnel de Croissance est de 1118,36 euros net, le revenu moyen d'un français est de 2410 euros brut par mois (Acoss 2012).

L'enquête nationale sur l'obésité et le surpoids, Obépi-Roche 2012, retrouve en France pour la population générale de plus de 18 ans que 49,2\% de sujet ont un poids normal, 3,5\% sont maigre, $32,3 \%$ sont en surpoids et $15 \%$ de français présentent une obèsité [166]. Notre population de 15 sujets, en ce qui concerne l'IMC, est globalement proche de la population générale.

Les chiffres de 1'Institut National de Prévention et d'Education pour la Santé estiment la première cigarette dans la population générale Française entre 13,5 et 14,5 ans.

L'observatoire français des drogues et toxicomanies a estimé en 2010 la première expérimentation de cannabis entre 11 et 17 ans.

Les dépenses de la population générale (Figure 15) et celles de notre échantillon (Figure 14) se répartissent avec la même hiérarchisation.

Figure 15: Composition en pourcentage des différents postes de la CMT en 2011 au niveau national

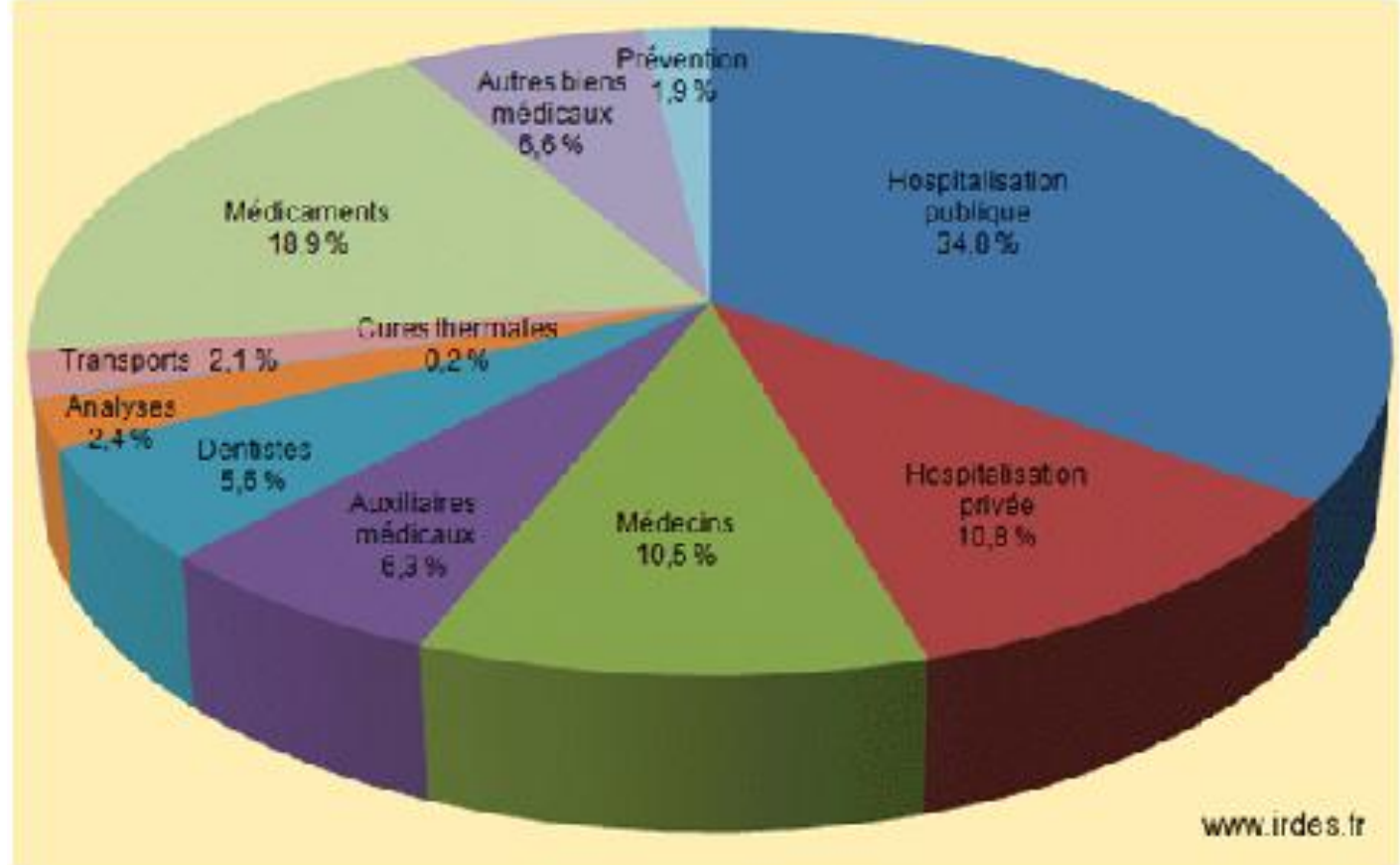

Source : Eco-Santé France 2012, d'après les données Drees, Comptes de la Santé [75] 


\subsubsection{Comparaison des moyennes annuelles nationales et des moyennes de l'échantillon}

Afin de comparer les moyennes de notre échantillon aux moyennes annuelles nationales pour les différents agrégats de dépenses (tableau 6), nous utiliserons les données nationales chiffrées pour l'année 2011 de Eco-santé France 2012 fournies par l'Institut de Recherche et Documentation en Economie de la Santé (IRDES). [79]

Nous constatons que les moyennes de Consommation de Soins Ambulatoires, de Consommation de Soins et de Biens Médicaux et surtout de Consommation Médicale Totale de notre population sont significativement différentes des moyennes nationales.

La différence entre les moyennes de notre population de Consommation de Soins Hospitaliers et de Consommation de Médicaments avec les moyennes nationales sont proches de la significativité statistique. Ce résultat est probablement dû au manque de puissance de notre étude en lien avec notre petit échantillon.

Tableau 6: Moyennes annuelles nationales de 2011 et moyennes de l'échantillon pour les différents agrégats de dépenses

\begin{tabular}{|c|c|c|c|c|c|}
\hline & $\begin{array}{c}\text { Moyenne } \\
\text { nationale } \\
2011 \\
\text { (Euros) }\end{array}$ & $\begin{array}{l}\text { Moyenne de } \\
\text { l'échantillon } \\
\text { (Euros) }\end{array}$ & $\begin{array}{c}\text { Rapport des } \\
\text { moyennes } \\
\text { (Echantillon/Nationale) }\end{array}$ & $\begin{array}{c}\text { Test-t } \\
\text { univarié : } \\
\text { Valeur de } \\
\mathbf{t}\end{array}$ & $\begin{array}{c}\text { valeur de } \\
\qquad p\end{array}$ \\
\hline $\begin{array}{l}\text { Consommation } \\
\text { de soins } \\
\text { hospitaliers }\end{array}$ & 1282,42 & 23972.125 & 18,69 & 2,42 & $p=0.029$ \\
\hline $\begin{array}{l}\text { Consommation } \\
\text { de soins } \\
\text { Ambulatoires }\end{array}$ & 700,75 & 3057,521 & 4,36 & 3,01 & $\mathrm{p}=0.009 *$ \\
\hline $\begin{array}{l}\text { Transport de } \\
\text { malade }\end{array}$ & 59,83 & 113,455 & 1,8 & 0,91 & $p=0.376$ \\
\hline $\begin{array}{l}\text { Consommation } \\
\text { de médicaments }\end{array}$ & 532,46 & 1854,68 & 3,48 & 2,02 & $p=0.062$ \\
\hline $\begin{array}{l}\text { Consommation } \\
\text { d'autres biens } \\
\text { médicaux }\end{array}$ & 186,88 & 571,371 & 3,05 & 1,29 & $p=0.216$ \\
\hline $\begin{array}{l}\text { Consommation } \\
\text { de soins et de } \\
\text { bien médicaux }\end{array}$ & 2762,34 & 29569,34 & 10,70 & 2,7 & $p=0.016 *$ \\
\hline $\begin{array}{l}\text { Consommation } \\
\text { médicale totale }\end{array}$ & 2814,77 & 29605,349 & 10,52 & 2,7 & $p=0.016^{*}$ \\
\hline
\end{tabular}

* statistiquement significatif 


\subsubsection{Dépenses en santé mentale}

Observer les chiffres liés aux pathologies somatiques en les comparant à la pathologie mentale, au sein des populations de patients, permet de souligner le poids économique de cette dernière.

En France, les coûts en santé mentale en 2007 (Chevreul 2012) représentaient 13,4 milliards d'euros soit 8\% de la Consommation de Soins et de Biens Médicaux. Il ne faut pas négliger les coûts indirects. En effet, la pathologie mentale est la première cause d'invalidité et le second motif d'arrêt de travail. On peut retrouver liés à la perte de productivité, environ 24,4 milliards d'euros et à la perte de qualité de vie 65 milliards. Le coût global approche les 109 milliards d'euros liés à la maladie mentale en France. [42]

Le diabète, représente 12,5 milliards d'euros par an en France. Un diabétique coûte alors 5300 euros annuel (chiffres InVS 2007).

Pour un patient hypertendu, les dépenses liées à sa pathologie sont de 422 euros par an. Ce qui représente 4,4 milliards d'euros au niveau national par an (chiffres de la sécurité sociale 2006).

En 2004, les dépenses d'un sujet atteint d'un cancer de l'appareil digestif étaient de 29000 euros annuel, 46000 pour un cancer hématologique et 19000 pour un cancer du sein. (Analyse économique des coûts du cancer en France, mars 2007, Institut nationale du cancer).

En 2006, les dépenses de santé d'un asthmatique étaient multipliées par 1,2 par rapport à la population générale, et étaient de l'ordre de 1275 euros par patient et par an. Le total des dépenses liées à l'asthme étaient alors de 2,4 milliards d'euros (chiffres IDRESS 2006).

La publication de Heijink R. de 2009 (tableau 7) nous donne le coût moyen par français des pathologies mais cette fois en population générale. [119] 
Tableau 7 : Coût et pourcentage des dépenses par pathologie et par français en 2002 (Le cours moyen de l'euro en 2002 était de 0,9 Dollars)

\begin{tabular}{|c|c|c|}
\hline & $\begin{array}{l}\text { Pourcentage par rapport } \\
\text { aux dépenses totales }\end{array}$ & $\begin{array}{c}\text { Coût par français en } \\
\text { Dollars US }\end{array}$ \\
\hline Maladies cardiovasculaires & $13,6 \%$ & 226 \\
\hline $\begin{array}{c}\text { Maladies de l'appareil } \\
\text { digestif }\end{array}$ & $13,4 \%$ & 222 \\
\hline $\begin{array}{l}\text { Troubles mentaux et du } \\
\text { comportement }\end{array}$ & $10,9 \%$ & $181(201 €)$ \\
\hline Tumeurs & $7,1 \%$ & 118 \\
\hline $\begin{array}{l}\text { Maladie de l’appareil } \\
\text { respiratoire }\end{array}$ & $7,1 \%$ & 119 \\
\hline $\begin{array}{c}\text { Maladie endocriniennes et } \\
\text { métaboliques }\end{array}$ & $4,3 \%$ & 71 \\
\hline ... & ... & ... \\
\hline Total & $100 \%$ & \\
\hline $\begin{array}{l}\text { Dépenses en soins et biens } \\
\text { médicaux en Dollars US. }\end{array}$ & & $\begin{array}{c}2817 \\
(2928 €)\end{array}$ \\
\hline
\end{tabular}

Source : Heijink R. 2009

Concernant les sujet victimes de violences sexuelles avant l'âge de 15 ans de notre étude, nous pouvons obtenir et analyser la part de leurs dépenses dédiées aux soins en santé mentale. Le coût des consultations psychiatriques, des hospitalisations en psychiatrie et des autres soins ambulatoires tels que les consultations chez un psychologue ou encore les prises en charge en hôpital de jour est alors associé.

Nous obtenons alors une dépense moyenne en santé mentale de 1539,54 euros (Coût maximum $=6$ 934,52 euros et un coût minimum $=204,35$ euros $)$.

Les dépenses de santé mentale représentent en moyenne 20,43\% des dépenses totales.

La répartition dans notre échantillon est schématisée figure 16. 


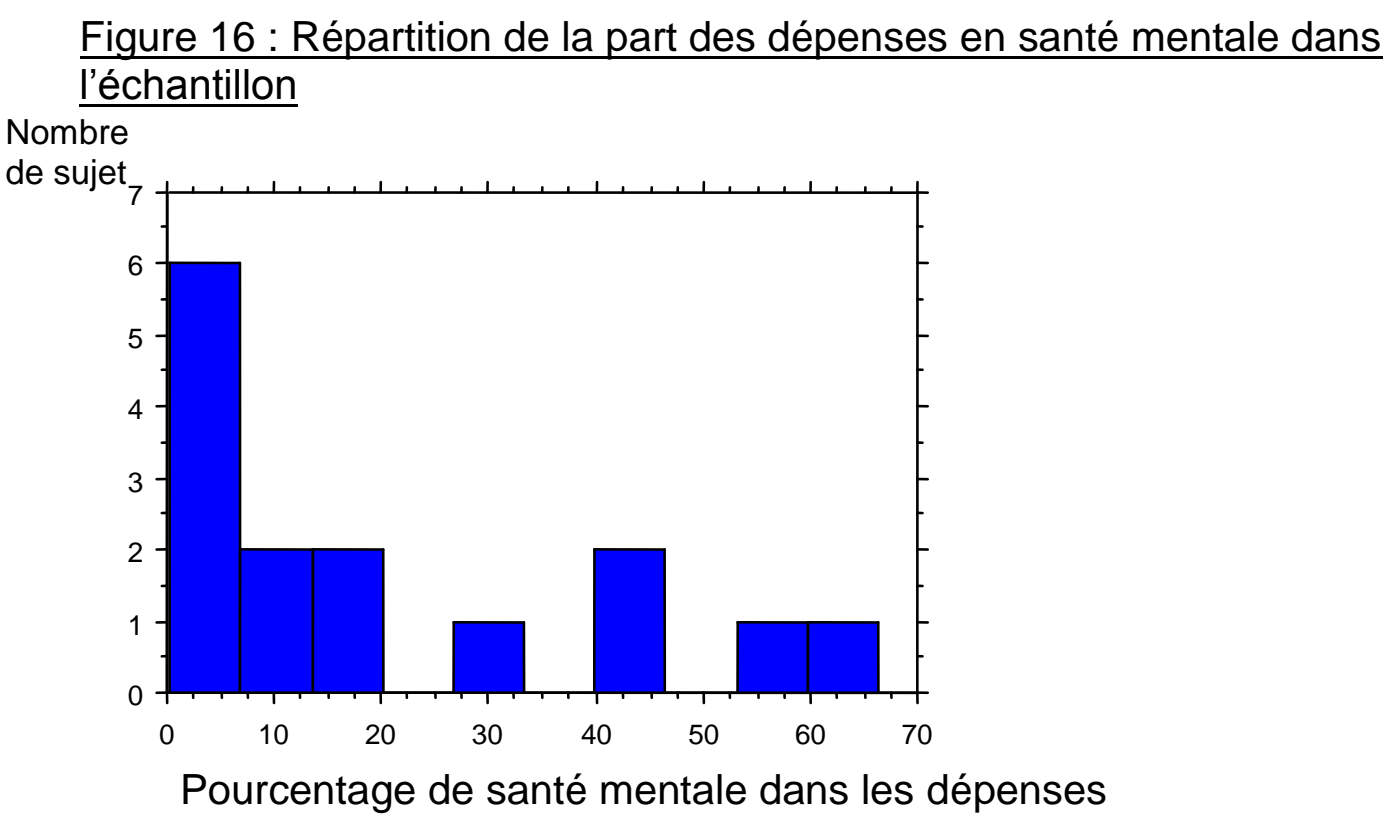

Nous pouvons alors comparer les dépenses annuelles en santé mentale de notre échantillon (1 539,54 euros) à celle de la population générale en France, qui est de 201 euros par français pour l'année 2002 (Heijink 2009). On obtient une différence extrêmement proche de la significativité statistique avec un $\mathrm{t}=2,76$ et $\mathrm{p}=0,015$.

Par déduction, on obtient les dépenses de santé hors santé mentale. Pour notre échantillon, la moyenne est de 28 033,61 euros et pour la population générale française en 2002, elle est de 2928 euros (Heijink 2009). Ses deux moyennes sont quasiment significativement différentes sur le plan statistique $(\mathrm{t}=2,6$ avec $\mathrm{p}=0,02)$.

\subsubsection{Recherche de facteurs pouvant influencer les dépenses}

- On ne retrouve pas de différence statistiquement significative de la consommation médicale totale et de la consommation de soins ambulatoires entre les sujets ayant été victimes de viol ou ceux ayant été victimes d'autre type d'agression sexuelle (Test Mann-Whitney $p=0,39$ et $\mathrm{p}=0,23)$.

- Il n'y a pas de lien statistiquement significatif entre les scores IES-R ou BDI avec la Consommation Médicale Totale ou encore la Consommation de Soins Ambulatoires. 
- Entre la Consommation Médicale Totale et l'âge au moment des faits, on ne retrouve pas de lien statistiquement significatif. Cependant, le manque de puissance peut encore une fois expliquer la non significativité statistique car on observe un Rho=0,5 pour $\mathrm{p}=0,06$.

- Nous ne retrouvons pas de lien statistiquement significatif entre l'âge au moment des faits et les autres postes de dépenses ni avec les scores IES-R et BDI.

- Il n’y a pas de différence en termes de Consommation Médicale Totale entre les sujets dont l'agresseur était un membre de la famille ou non, ou même simplement une connaissance de la victime.

- Le lien entre l'âge actuel du sujet et la CMT n'est pas statistiquement significatif, cependant on obtient un $\mathrm{Rho}=-0,05$ et $\mathrm{p}=0,047$. On pourrait quasiment dire que plus le sujet est jeune plus le coût est important.

- Pas de lien entre le délai de prise en charge et la Consommation Médicale Totale ou encore le coût des soins en santé mentale.

- D'autres résultats sont proches de la significativité statistique :

Entre le coût des soins en santé mentale et l'âge de l'agresseur au moment des faits on obtient Rho $=-0,57$ et $\mathrm{p}=0,033$.

Entre le coût des soins en santé mentale et l'âge du patient au moment de sa prise en charge, Rho $=-0,49$ et $\mathrm{p}=0,06$.

Entre la part du coût en santé mentale et le score de BECK, Rho=0,50 et p=0,057.

- Nous avons voulu établir un score de gravité regroupant, l'âge du sujet au moment des faits, si l'agresseur était un membre de la famille, la survenue d'autres évènements traumatiques au cours de la vie et le Score IES-R.

- Nous n'avons pas obtenu de lien statistiquement significatif entre les scores de gravité et les dépenses mais nous pouvons observer la répartition de ce score dans notre population figure 17. 


\section{Figure 17: Nombre de sujet par score de gravité obtenu}

Nombre de

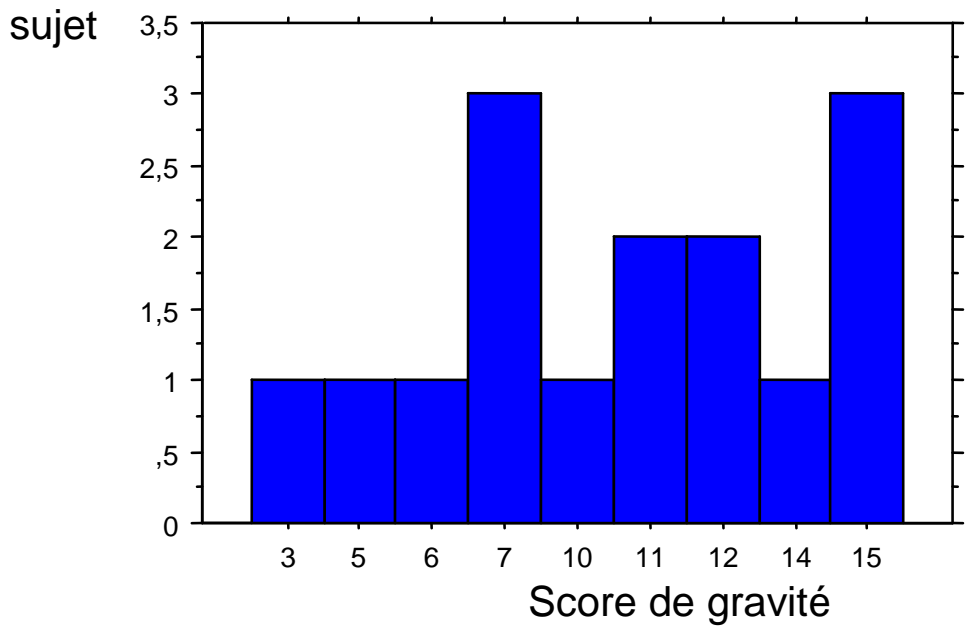

\section{DISCUSSION}

Les sujets pris en charge à l'hôpital Tenon ayant été victimes de violences sexuelles avant 15 ans dépensent bien plus que la population française générale en ce qui concerne les Soins Ambulatoires mais aussi la Consommation de Soins et Biens Médicaux et la Consommation Médicale Totale. Pour certains agrégats, un facteur 10 peut être calculé entre les dépenses de nos sujets et celle d'un français moyen. Le même constat est fait pour les dépenses de santé mentale.

D'autres résultats, tout aussi intéressants ne sont que proches de la significativité. Nous pouvons alors mettre en avant le manque de puissance de l'étude dû à notre petit échantillon. Notre population semble dépenser davantage que la population générale en soins hospitaliers et en médicaments. Les coûts en santé mentale semblent être majorés lorsque le sujet est jeune au moment de la prise en charge et aussi lorsque l'agresseur est jeune au moment des faits. La consommation médicale totale pourrait être plus importante lorsque le sujet interrogé est jeune. L'intensité de la symptomatologie dépressive, plus que celle de l'ESPT, semble majorer les dépenses. 
Les résultats négatifs se doivent d'être mis en évidence. Ainsi, le lien à l'agresseur et le type de violences sexuelles n'ont pas de répercussions sur les dépenses de santé de nos patients une fois devenus adultes.

Nos résultats, bien que certains ayant une significativité statistique, ne peuvent être généralisés. En effet, notre étude souffre de diverses limites. Cependant, de par notre position, nous pouvions difficilement les contourner.

- Tout d'abord, le recrutement de nos sujets peut être discuté. Il engendre de toute évidence des biais. L'échantillon recruté fait partie de la population générale intégrée dans la comptabilité nationale. De plus, nos sujets ne sont issus que d'un unique centre, très spécialisé et faisant partie d'une région où l'offre de soins n'est pas représentative au niveau national.

- Le recueil de données, et particulièrement celui des dépenses, est tout aussi discutable en termes de fiabilité, car reposant sur les dires des patients, faisant appel à leur mémoire ainsi qu'à leur bonne volonté. Nous ne pouvons omettre une possible surestimation pour certains, possiblement liée à leur besoin de reconnaissance et de réparation. A l'opposé, une sous estimation est possible par des sujets ayant des difficultés à retracer leur parcours de soins, ou alors, bien qu'ayant accepté l'inclusion, se trouvent réticents devant la densité des questions.

Cependant, des résultats comme celui de la hiérarchisation des postes de dépenses, nous permettent d'être globalement satisfaits des investigations et des calculs, renforçant la pertinence du questionnaire.

Des critiques peuvent toutefois être émises sur la qualité des questionnaires. Des points supplémentaires auraient pu être évalués, notamment au niveau clinique avec des échelles plus poussées. Nous avions craint le caractère rébarbatif pour les sujets de la première partie, donc voulu alléger les auto-évaluations. C'était sans compter sur la disponibilité des sujets inclus qui à postériori nous ont paru en capacité de répondre à une évaluation clinique plus aboutie. Nous aurions peut être pu montrer une influence de l'état de santé psychique des patients sur leur dépenses, résultats qui manquent de manière indiscutable à notre étude.

Quantifier les coûts en médecine préventive à notre échelle a été l'une des difficultés majeures rencontrées. Certaines cliniques ont pu aussi se montrer réticentes à nous donner leurs tarifs. 
Il nous semble important de rapporter les remarques des sujets interrogés. Outre pour certains le caractère incongru d'une quantification des dépenses, ils ont pu nous interpeller concernant les associations auxquelles ils ont recours. Leur fonctionnement basé sur le bénévolat est financé majoritairement grâce aux dons de mécènes et quelques subventions de 1'Etat. Il nous était évidemment impossible de quantifier ce coût bien réel, qu'il ne faut pourtant pas omettre de citer. Ces remarques mettent en avant l'importance du soutien social pour ce type de patients.

Nous avons essentiellement limité notre étude aux dépenses liées à la santé que l'on qualifie de coût direct. Cependant, nous voyons clairement que les études françaises sur la santé mentale ne ciblant pas de pathologie particulière, s'intéressent aussi aux dépenses indirectes. Nous n'avons pu réaliser qu'une ébauche de cette investigation en cherchant à quantifier les arrêts de travail et les indemnités journalières faisant partie intégrante de la perte de productivité. Il semble donc indispensable de pouvoir aussi évaluer l'impact des violences sexuelles pour ces coûts indirects, touchant les domaines sociaux et juridiques comme l'ont fait les études internationales citées précédemment.

\section{CONCLUSION DE L'ETUDE}

Malgré les multiples biais cités précédemment, ce travail mené avec très peu de moyens et à très faible échelle, met en exergue le poids économique des conséquences à l'âge adulte, de violences sexuelles avant 15 ans. Nous le constatons clairement en termes de santé mentale et de dépense de santé globale, mais l'étude nous laisse aussi supposer un impact sociétal considérable.

La faisabilité des recherches sur ce thème est démontrée et ainsi une étude à plus grande échelle peut être justifiée. Elle permettrait d'affiner les résultats précédents et d'ouvrir la porte des coûts indirects pour ce type de patients. Pourquoi ne pas tenir aussi compte du coût sur l'entourage du sujet concerné par les violences sexuelles? 


\section{CINQUIEME PARTIE}

\section{VIGNETTES CLINIQUES}




\section{Mlle L.}

Il s'agit de la première patiente que j'ai été amenée à prendre en charge dans le service de psychotraumatologie de l'Hôpital Tenon en mai 2010.

Mlle L. est une patiente de 37 ans, adressée en hospitalisation par son psychiatre ainsi que sa psychologue, pour une symptomatologie anxio-dépressive avec des idées suicidaires.

Dès son arrivée dans l'unité, nous sommes frappés par la fragilité patente de Mlle L., jeune femme gracile, blonde aux yeux clairs, le teint blafard, osant tout juste s'adresser aux soignants d'une voix blanche. Nous apprenons alors qu'elle est célibataire, sans enfant, seule fille d'une fratrie de quatre enfants, dont elle est la benjamine. Son père est décédé à l'âge de 67 ans des suites d'un cancer du rectum voilà 5 ans et dit ne plus avoir de contact avec sa mère depuis un an. Elle exerce la profession d'employée dans une compagnie d'assurances depuis 1996 et bénéficie d'un mi-temps thérapeutique depuis 2 ans.

Voilà un an et demi que Mlle L. est suivie par un psychiatre libéral. Elle consulte une psychologue dans un centre anti-douleur depuis 2 ans, ainsi qu'un algologue pour une fibromyalgie, dont le diagnostic a été porté en 2006. Sa seule hospitalisation en psychiatrie était au mois d'octobre 2009, en clinique, pendant sept semaines, pour un épisode dépressif majeur évoluant depuis plusieurs mois.

Grâce au courrier du psychiatre, nous connaissons le motif de cette prise en charge qu'il a voulu spécialisée en psychotraumatologie. Mlle L. ne parvient pas à l'évoquer, elle est immédiatement en pleurs et prise de tremblements. En effet, alors qu'elle se trouvait en clinique six mois auparavant, elle a révélé à une soignante, dont elle dit avoir perçue l'empathie, avoir été victime d'agressions sexuelles à l'âge de 9 ans par son cousin alors âgé de 15 ans ; la détresse de la patiente n'allant depuis lors que crescendo.

Dans ses antécédents, nous retrouvons une notion de traitement par d'Amitriptyline (LAROXYL®), stoppé rapidement pour des épisodes d'hypotensions orthostatiques, de la Miansérine (ATHYMIL®), là aussi mal tolérée et enfin de la Duloxétine (CYMBALTA®) pendant 6 mois, décrite comme efficace sur ses douleurs mais sans amélioration de l'humeur. Elle n'a par ailleurs aucune consommation de toxiques. 
L'ordonnance habituelle de Mlle L comprend 11 molécules : Sertraline( ZOLOFT®) 200mg/j, Cyamémazine (TERCIAN®)100mg/j, Alprazolam (XANAX®) 0,75mg/j, Tetrazepam (MYOLASTAN®)150 mg/j, Prégabaline (LYRICA®) 175mg/j, Paracétamol, Ibuprofène, Zopiclone (IMOVANE®), Pantoprazole (INIPOMP®), Chlorhydrate d'héptaminol (HEPT-A-MYL®), Anétholtrithione (SULFARLEM®).

Cette longue liste de traitements systématiques, traduction de la souffrance psychique et physique de Mlle L., met en évidence la difficulté des différents intervenants à trouver une solution autre que médicamenteuse pour la soulager quitte à multiplier les molécules.

Au fil des entretiens, aussi bien médicaux que psychologiques ou infirmiers, nous apprenons à faire sa connaissance. Elle arrive à être en confiance avec une équipe bienveillante, à son écoute et abordant à son rythme son histoire de vie.

Dans l'histoire familiale, on retrouve l'alcoolo-dépendance de son frère H., dont elle se dit proche et qu'elle cherche à tout prix à protéger, une tante maternelle qu'elle décrit comme dépressive et ce cousin T., auteur des agressions décédé à l'âge de 31 ans par suicide il y a maintenant 13 ans. Ses antécédents familiaux sont schématisés grâce au génogramme réalisé figure 18 .

\section{Figure 18: Génogramme de Mlle L}

$$
\begin{aligned}
& \text { - Une tante paternelle } \\
& \text { décrite comme dépressive }
\end{aligned}
$$

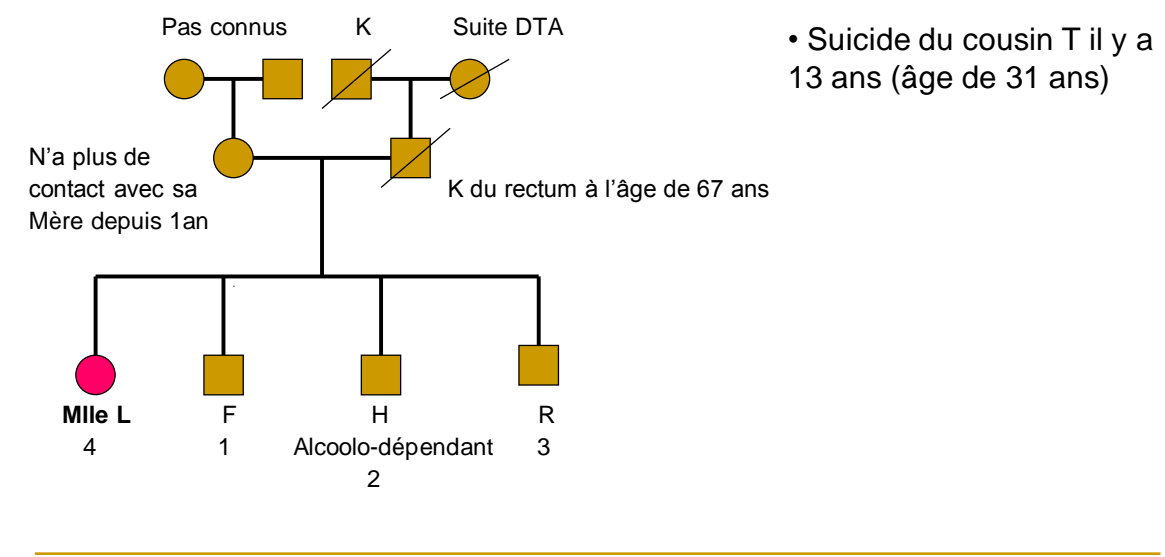


Nous pouvons percevoir au travers de son discours, une idéalisation du père dont le décès a été un accélérateur de sa grande détresse, son frère ainé $F$., faisant dès lors office de figure paternelle de substitution. A l'inverse, elle rejette totalement sa mère, la décrivant comme absente et en qui elle n'aurait «jamais eu confiance».

Les entretiens avec Mlle L. doivent se faire à des moments choisis et chaque intervention pesée, tant elle parait vulnérable, sursautant à chaque intrusion dans sa chambre, puis assise tremblante au bord de son lit, jouant nerveusement avec ses doigts, les yeux fixant le sol.

La symptomatologie anxieuse est au premier plan et les éléments dépressifs prégnants. Triste et de plus en plus isolée socialement, elle passe la majorité de son temps à son domicile, au lit, ruminant ses idées suicidaires, s'imaginant se précipiter sous un train ou encore provoquer un accident de la voie publique.

Les symptômes de stress post-traumatiques sont de toute évidence importants. Elle décrit comme majoritairement invalidants au quotidien ses cauchemars, toujours empreints de violence avec des réveils multiples, des reviviscences accompagnées de signes neurovégétatifs, sueur et tachycardie et une hypervigilance quasi constante. Un trouble du comportement alimentaire sera mis en évidence, avec une prise de poids conséquente, plus 7 kilos en 5 mois, fait de «pulsions alimentaires pour éviter les images », sans comportement compensatoire.

Elle pourra verbaliser sa colère à l'encontre de sa mère « absente », sa rancœur pour sa grandmère, chez laquelle les faits se déroulaient, une difficulté de positionnement vis-à-vis de ses frères, mais surtout sa culpabilité, sentiment la submergeant à l'évocation de son silence alors même que sa mère l'interrogeait suite aux plaintes d'une autre fillette pour les mêmes faits.

Progressivement, elle parviendra à mettre en mots son ressenti et même, de manière moins allusive qu'au départ, le déroulement des faits, toujours avec pudeur.

Cependant, il est plus aisé pour elle de s'exprimer par écrit, elle nous fera parvenir les deux documents ci-dessous. L'un dactylographié (document 1), antérieur à l'hospitalisation et l'autre rédigé pendant l'hospitalisation (document 2). 


\section{Document 1}

Le 18 mars 2010

Voilà comment tout cela se passait.

J'avais 9 ans, lui 15. Lui, mon cousin, T

C'était toujours chez ma grand-mère, le mercredi. Mes frères qui étaient sensiblement $d u$ même âge que lui ne venaient déjà plus chez elle pour passer le mercredi. Ils restaient à la maison ou sortaient avec des copains.

Mais lui, non, il venait chez mes grands-parents.

Le « pourquoi ? » ne se pose même pas. La raison était évidente.

Ma grand-mère avait fait d'une pièce un salon pour recevoir du monde. Un espace de vie pour elle aussi et mon grand-père.

Cette pièce n'était pas un espace de vie pour tout le monde.

Pour moi c'était juste un lieu de violence silencieuse.

Il transformait cette pièce en sorte de camping, afin de jouer disait-il à ma grand-mère.

Mon grand-père lui travaillait et ne voyait donc pas tout ça.

Pour se cacher, pour nous cacher, il alignait des chaises en les recouvrant de draps, afin de former une sorte de tente.

Tout se passait en dessous de cette « tente » fabriquée par ses soins.

Je me souviens que ma grand-mère disait « quel bazar vous mettez, vous pourriez trouver

d'autres moyens de vous amuser ! ».

Et là, allongée par terre, il faisait ce qu'il avait à faire. Tellement de choses.

C'est la première fois, même par écrit, que je crois que je vais réussir à décrire ces choses. Ces choses qui envahissent ma tête.

J'étais une petite fille, donc souvent en jupe, ce qui lui facilitait la tâche. Et puis je me souviens qu'il me demandait de me mettre en jupe, et moi j'exécutais.

Il soulevait donc ma jupe, sans me déshabiller complètement, baissait ma culotte et enfonçait son sexe dans le mien.

Il mettait aussi son sexe dans ma bouche.

Mais de l'ensemble des ces actes, je crois que le pire était lorsqu'il m'embrassait.

Même ça il me l'a volé. Mon premier baiser.

Je me souviens qu'après j'avais le besoin d'aller à la salle de bain.

Et puis, on allait prendre notre goûter comme si rien ne s'était passé.

Mais certaines fois, c'était pire. Une chose se passait sous les yeux de ma grand-mère qui ne voyait rien. Il faut dire qu'elle était en adoration devant mon cousin.

C'était dans la salle à manger, assis autour de la table. Il me prenait sur ses genoux et passait ses mains sous ma jupe.

Pourquoi avoir laissé faire ? Pourquoi ne pas avoir dit à mère « oui, il se passe des choses » lorsqu'elle me l'a demandé ?

Aujourd'hui je n'éprouve que du dégoût.

Mes sentiments me dégoûtent, mon silence aussi, mon corps mes pensées. 
Document 2

$$
\text { L........ 9/0S/2010. }
$$

Les images sont differentes depuis que je suis ici.

Avant d'ètre à l' hopital je revoyais sans cesse les actes, je seatais son odeur...

In limage est fwee. If me regarde, pend plaisir à me vait lic en se disant que non sestement if m' a fait mat pendant l'enfance et qu'aujourd'hui c'est moi qu' on enferme à f'hopital, pour me punir - Je me sens "enfermèe pour un acte que fui m'a fait subir.

Son regard ne me fäche pas un seul instant.

On me demande souvent si je faùs des cauchemars. Si dui, je ne méen saviens jas je ne êveille claque nuit britalement, en sueur ed angoissée. Par contre, je rêve que je suis avec ma famitle et à ce moment. lá coussi je me revertle tres angoissee. Surement a cause de toute cethe peur à deviler mon secret.

Parler ou ne pus paster. je este à souffoir. Si je garde le silence ma famille parra evider de suuffir avec mo: En mêne denps je n'errive plus à vivre avec ce passé - Alors, quelle est ka solution? Les scénarios de suicide ne sont plus presents comme il y a quelques semaines, mais fidée de mort est tojours prejente. La mort serait le symbote de $f_{a}$ fin de cette souffrance qui restera tajgurs présente. Je me sens lellement éprisée que mâme d’alter ou bout de cet acte... Je n'ai pas cette force, ce courage. It pourtant, jáa Pimpression que je vis un combat perdu davance.

olon but de P'hospitalisction est le suivant: etre apaisee, ne plus voir ces inages, mas caussi et surtort savoir quette attitude avoir avec ma famille.

j’ai trés peur pour un de mes frères, Herve. Il était bres proches de mon cousin. En revelant le passé il va temiblement souffirir.

132 
Après quelques semaines d'hospitalisation, elle nous demandera d'organiser un rendez-vous en présence de son frère ainé $\mathrm{F}$. et de sa belle sœur, dont elle se dit proche. Elle les décrit comme soutenants, mais lui exprimant leur désemparement, ne connaissant le motif de son mal-être. Mlle L. nous fait aussi part de sa peur de l'après, peur de ce que ses deux proches feront de «ce secret » qui jusqu'alors lui appartenait. Lors de cet entretien, elle leur explique les faits comme elle le souhaitait. Le frère, attentif et bienveillant, tout comme sa femme, sera dans un premier temps totalement silencieux, comme sonné par cette révélation, versant lui aussi des larmes mais reprenant le dessus, exprimant sa volonté de poursuivre le soutien qu'il peut apporter à sa soeur.

Mlle L. attachant beaucoup d'importance à ses traitements médicamenteux, seules quelques modifications ont pu être réalisées et après de longues explications des bénéfices attendus et des effets secondaires possibles. Ainsi, l'antidépresseur sera modifié, Sertraline (ZOLOFT®) contre Venlafaxine (EFFEXOR®). Les antalgiques, Paracétamol et Ibuprofène ne seront plus en prises systématiques. Les douleurs en lien avec sa fibromyalgie pour lesquelles elle bénéficie de kinésithérapie s’atténuant, elle en modérera la consommation.

Mlle L. restera hospitalisée six semaines dans le service, avec à la sortie un projet de maison de convalescence puis de suivi ambulatoire par son psychiatre habituel mais aussi de poursuite du travail psychothérapeutique débuté avec une des psychologues du service de Tenon.

Cette hospitalisation lui aura permis de mettre à distance ses idées suicidaires, de se confier à deux personnes lui apportant un soutien familial indispensable, son frère et sa belle-sœur, une nette amélioration de sa symptomatologie algique, une partielle d'adaptation du traitement mais compliquée par la réticence de Mlle L.

Grâce à une ébauche d'élaboration, la jeune femme fragile et terne avait gagné quelques forces psychiques et physiques se permettant d'esquisser des sourires que jusqu'alors nous ne lui connaissions pas. 
A titre informatif, à son arrivée, l'HAD (Hospital Anxiety and depression) évaluait une anxiété à 15/21 et la dépression à 16/21, l'IES-R (Impact of Event Scale-Revised) quant à elle était de 57. A la sortie, ils seront de 11 pour l'anxiété, 13 pour la dépression et 51 pour l'IES$\mathrm{R}$.

Cependant, son parcours allait encore être long, plusieurs hospitalisations suivront celle-ci. Les difficultés familiales, professionnelles et sociales, allaient refaire surface. Sa force de caractère toute différente et sa passivité écartée, pouvaient nous faire craindre un passage à l'acte en cas de nouvelles idées suicidaires, nous devions alors l'aider à utiliser cette détermination dans le chemin vers la résilience.

\section{Bilan de Mlle L.}

Le cas de Mlle L, met en évidence la grande intrication entre douleur psychique et physique avec la difficile prise en charge médicamenteuse.

Mais surtout, cette observation montre le rôle majeur de l'hospitalisation pour ces patients en situation de crise. L'objectif primaire reste le désamorçage de la phase aigue, mais secondairement, elle peut être le temps de renouer et d'améliorer soutiens familial et social indispensables pour l'amélioration des symptômes. Dans ce sens, les groupes de paroles fréquemment proposés dans les services d'hospitalisation, médiation permettant l'échange d'expériences communes, peuvent contribuer à une ébauche de lien social. 


\section{Mlle H. Laurie}

Mlle H Laurie est une jeune adolescente prise en charge en pédopsychiatrie à Orsay que j'ai été amenée à suivre lors de mon stage d'un an dans ce service en 2011.

Laurie, 14 ans a été adressée au Centre d'Accueil d'Urgence et de Soins par les urgences du Centre Hospitalier, ainsi que sur les conseils de son pédiatre.

La demande de consultation est faite par la mère auprès du secrétariat. Les motifs rapportés sont : des idées suicidaires, une variabilité de l'humeur, des troubles du sommeil ainsi que des difficultés scolaires.

\section{Premier entretien d'évaluation le 9/02/2011}

Laurie est en classe de $4^{\text {ème }}$, dernière d'une fratrie de trois, composée de 2 grands frères J. (20 ans) et D. (19 ans). Les parents sont séparés depuis que Laurie a 6 ans. La procédure de divorce est toujours en cours.

\section{$\underline{\text { 1. Histoire familiale }}$}

Figure 19: Génogramme de Mlle H.

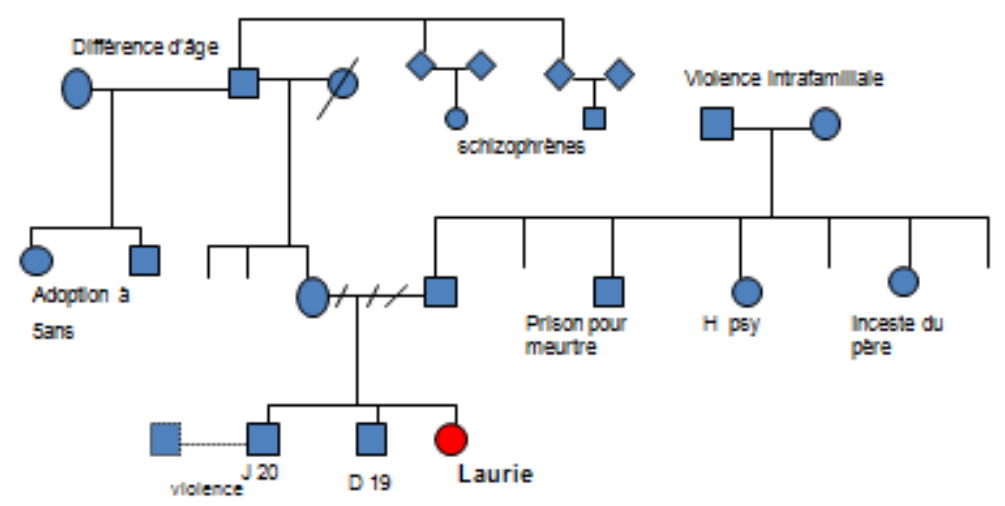


Au delà du divorce des parents, le génogramme figure 19, partant de Laurie et ses frères, met en évidence des antécédents familiaux notables. La branche paternelle est marquée par une violence intrafamiliale. Une notion de violence conjugale des grands-parents, un oncle purgeant une peine de prison pour meurtre, une tante ayant des antécédents d'hospitalisation en psychiatrie et une autre tante ayant porté plainte bien après sa majorité contre son père pour agressions sexuelles.

La branche maternelle n'est pas en reste, avec deux cousins de la mère diagnostiqués schizophrènes et un grand père veuf, puis remarié à une jeune femme de vingt ans de moins, le couple ayant adopté il y a un an, deux enfants de cinq ans.

Le frère ainé de Laurie, homosexuel, semble lui aussi vivre au sein de son couple un climat de violence verbale et physique.

\section{$\underline{\text { 2. L'histoire de vie de Laurie }}$}

La mère nous fait d'emblée part des attouchements dont Laurie aurait été victime entre 4 et 5 ans par son père. Suite à la révélation des faits par Laurie, madame quitte immédiatement le domicile avec ses enfants. Un premier dépôt de plainte pour attouchements sexuels est enregistré mais sera classé sans suite, les 2 frères contestant les propos de Laurie.

Le frère ainé J., est suivi à cette époque en pédopsychiatrie par la chef de service le Dr P. Alors âgé de 12 ans, il présentait des éléments dépressifs avec des mises en danger à répétition, deux tentatives de suicide et des périodes d'anorexie mentale. A son tour, J. portera plainte à l'âge de 18 ans (en 2009) contre son père pour viol. Le dossier de Laurie sera alors ré-ouvert.

Quant au deuxième frère, il affirme jusqu'à ce jour, ne pas avoir été victime du père.

C'est alors dans ce contexte de procédure judiciaire avec le procès d'assises débutant dans dix jours, que la prise en charge de Laurie débute au CAUS. 


\section{Les antécédents de Laurie}

Dans les antécédents médicaux sont à noter des infections urinaires à répétition dans l'enfance. Depuis la classe de CM1, Laurie consulte une orthophoniste pour dyslexie, dyscalculie et dysorthographie.

Elle bénéficie d'aides médico-sociales. Une mesure d'AEMO judiciaire (Action Educative en Milieu Ouvert) a été mise en place depuis 2001, grâce à laquelle elle reçoit l'aide d'une éducatrice et a depuis 6 mois quelques entretiens avec une psychologue, avant les étapes majeures de la procédure judiciaire. Un PAI (Projet d'Accueil Individualisé) a été mis en place cette année (en $4^{\text {ème }}$ ), à la demande du pédiatre et de l'orthophoniste avec un allègement des heures de cours et des devoirs maison.

Le pédiatre, devant les manifestations anxieuses de Laurie, a déjà prescrit de manière successive : phytothérapie avec de l’Euphytose ${ }^{\circledR}$, homéopathie avec du Sédatif PC $®$ ainsi que de l’Hydroxysine (Atarax $®)$ sans obtenir d'amélioration notable.

\section{L'entretien avec Laurie.}

$\mathrm{Au}$ premier entretien, Laurie se présente comme extravertie, se définissant comme «métalleuse ». En effet, elle est vêtue uniquement de noir avec un maquillage très prononcé. Ses cheveux sont blonds et longs, une grande mèche lui couvrant la quasi moitié du visage. Frange dans laquelle elle passe continuellement les doigts s'assurant d'un positionnement parfait.

Laurie est d'emblée très volubile. Même en présence de sa mère en début d'entretien, elle ne lui laisse que très peu la parole. On peut alors noter, une tachypsychie avec fuite des idées, une familiarité ainsi qu'une exaltation de l'humeur. Son discours logorrhéique, est centré sur ses multiples plaintes, majoritairement d'ordre somatique, douleurs aux membres inférieurs, asthénie, de fréquentes céphalées et des douleurs abdominales. Elle présente ses idées suicidaires, dont elle parle au passé, mais avec une dimension théâtrale non négligeable et sans symptomatologie dépressive associée. Non sans une certaine fierté, elle dit avoir fait part de ses scénarii «à tous ses amis », «je pensais sauter sous le RER ou du pont », «j'ai aussi parlé d'une corde ». Elle montre ses cicatrices de scarifications qu'elle compare à celles de son frère $\mathrm{J}$. 
Laurie nous fait part de ses «sautes d'humeur», son impulsivité, son intolérance à la frustration ainsi qu'une grande irritabilité se manifestant contre sa mère. Eléments qu'elle décrit parfaitement, s'en plaignant et espérant trouver des solutions.

Menant l'entretien et essayant de contenir son flot de paroles, des symptômes d'ESPT sont retrouvés. Les détails de ceux-ci ne seront pas d'emblée abordés, mais semblent très invalidants, à type de troubles du sommeil (difficultés d'endormissement et cauchemars), troubles de la concentration et de flashbacks.

Laurie évoquera à peine son père lors de ce premier entretien, le peu qu'elle en dira sera avec un sourire largement défensif.

Quand elle décrit ses frères, elle tient des propos très durs au sujet du second, « lui n'a pas vécu les mêmes choses que J. et moi, il ne comprend rien ». A l'opposé, elle calque la présentation de son histoire et de ses symptômes sur ceux de son frère aîné. Il semble au cœur de ses inquiétudes ce jour là. Elle le décrit comme fragile, ne sachant pas se protéger, pour preuve son compagnon actuel qui serait selon elle, violent.

Sans transition et de manière totalement décousue, elle évoque le décès il y a 11 mois d'un ami dans un accident de la route, ainsi que de son cousin d'un cancer puis le suicide d'un autre ami il y a 3 mois. Ces trois évènements sont rapportés sans aucun affect, comme des faits anodins. Il semble alors que ce détachement permette de ne laisser par la suite aucune trace émotionnelle de ce récit : manifestation prégnante d'évitement chez Laurie.

Elle ne rapporte pas de consommation de toxiques, cependant elle évoquera un épisode d'alcoolisation aigue récent, seule au domicile.

\section{$\underline{\text { 5. Résumé Clinique de cet entretien }}$}

Ce premier contact avec Laurie, nous permet de retenir des symptômes d'hypomanie avec tachypsychie, logorrhée, exaltation de l'humeur, familiarité, coq à l'âne. Certains éléments permettent l'ébauche d'un trouble de personnalité, non encore défini à son âge, mais évoquant déjà un trouble de type limite ou histrionique. 
Les symptômes d'ESPT, bien qu'explorés partiellement, sont déjà manifestes avec des cauchemars et des flashbacks, des symptômes neurovégétatifs, des troubles du sommeil, une irritabilité, mais aussi des conduites d'évitement qui semblent importantes chez Laurie.

A noter aussi, les relations à ses frères absolument opposées, l'une projective et l'autre rejetante.

\section{Les entretiens suivants de février à octobre}

Les entretiens seront fixés au rythme d'un par semaine. Cependant, ils ne s'avéreront pas réguliers, Laurie ou sa mère oubliant un rendez vous sur 3 environ.

Toutefois, Laurie sera rapidement dans l'élaboration et plus authentique, l'alliance s'établissant rapidement.

La prise en charge de Laurie est rythmée par la procédure judiciaire, avec une nécessaire intensification de l'écoute et du soutien à l'approche des rencontres avec son avocat, des audiences avec le juge et surtout des confrontations avec le père.

\section{Traitements médicamenteux de Laurie :}

Pour Laurie, les premiers symptômes jugés handicapants, sont des attaques de panique, se majorant depuis peu. Celles-ci peuvent survenir aussi bien au domicile qu'en cours. Suite à l'échec de l'Hydroxysine essayé par le généraliste, de l'Alprazolam sera prescrit si besoin et de manière uniquement temporaire. En quelques semaines, ses attaques de paniques céderont.

La variabilité d'humeur de Laurie est facilement repérable d'un entretien à l'autre de par sa présentation vestimentaire et son maquillage changeant, mais aussi par ses capacités d'élaboration et de concentration fluctuantes. Laurie perçoit et décrit clairement cette labilité thymique «handicapante et épuisante dans son quotidien », ne facilitant pas les interactions sociales et familiales ainsi que son travail scolaire. Nous abordons alors la possibilité d'un traitement thymorégulateur. 
Un mois après le début du suivi, Laurie fera un passage aux urgences du Centre Hospitalier avec comme demande : «je veux être internée en psychiatrie pour qu'on s'occupe de moi et qu'on me soutienne ». Formulation à la fois provocatrice et désarmante comme sait si bien le faire Laurie. Elle sera reçue par le pédopsychiatre d'astreinte, il notera une grande facticité des affects avec des éléments d'hypomanie.

La question du traitement est alors de nouveau posée. Il lui sera prescrit un thymorégulateur, Valpromide, après avoir informé sa mère et elle-même des modalités du traitement donc de l'observance, du suivi biologique, des effets secondaires possibles et des bénéfices attendus.

Le traitement sera rapidement efficace sur la symptomatologie, avec une bonne observance car elle avoue en ressentir les bénéfices. «Je suis moins speed», «je me maquille moins ». Le même constat est rapporté par son entourage.

Après deux semaines, Laurie présentera des douleurs abdominales avec nausées et vomissements. Le traitement sera stoppé, suspectant une mauvaise tolérance. Le bilan biologique le confirmera, montrant une perturbation hépatique modérée et isolée.

La variabilité d'humeur de Laurie sera de nouveau présente une semaine après l'arrêt du traitement, mais de manière plus modérée.

Un traitement par antipsychotique est alors discuté mais ne sera pas prescrit, le suivi devenant trop irrégulier par la suite.

\section{Les éléments cliniques importants}

Les mises en danger à répétition de Laurie :

Notons un épisode d'agression physique en $6^{\text {ème }}$ par un groupe d'élèves de $3^{\text {ème }}$ En plus des multiples menaces de passage à l'acte auto-agressif déjà évoquées, Laurie provoque verbalement les élèves du collège. S'en suivent des menaces régulières qui peuvent évoluer en bagarres. «Je dois frapper pour me protéger» »

Ces éléments créant un climat d'insécurité et d'hypervigilance au quotidien aussi bien dans les transports que les lieux publics. 


\section{Son rapport au corps :}

Elle ne peut décrire que son visage. Explique son maquillage, ses piercings et sa mèche pour cacher la ressemblance qu'elle dit avoir avec son père. "Quand je me regarde dans un miroir, je me dis que c'est un monstre qui m'a faite. »

\section{.L'identification projective à son frère $\mathrm{J}$.}

Laurie à un rapport à son frère J. plus que complexe. Il passe d'un statut de figure d'autorité à celui de protégé. Ambivalence, probablement induite pour une part par l'attitude de celui-ci mais induisant une confusion des rôles chez la patiente.

Dans un même temps, elle peut dire de lui : «il m'insulte au sujet de mes tenues », «il se prend pour mon père » mais aussi, «il a le droit, c'est mon frère et il souffre ». « Moi c'est moins grave, c'est pour mon frère que je veux qu'il paye » en parlant de son père. Un commentaire de Laurie montrera une ébauche de réflexion : «j'utilise peut être mon frère comme prétexte. »

Lors d'une visite de J. au domicile au cours d'un week-end, ils auront une altercation. Selon Laurie, il aurait essayé de la gifler. Elle s'autorisera à exprimer sa rancœur contre lui : «il ne m'a pas protégée quand il fallait », «il m'a menti petite en disant que mon père était quelqu'un de bien. » Cet épisode marquera une phase de rejet de son frère avec une différenciation de leurs symptômes respectifs.

\section{. Les « troubles du comportement alimentaire»}

A la suite d'une énième dispute avec sa mère, Laurie décrira des épisodes de vomissements provoqués sur un week-end. Au sujet de son agressivité verbale, elle dira: «celui qui me parlait s'en prenait plein la gueule !»

Après certaines contrariétés, Laurie sera dans le refus de s'alimenter mais sur des périodes ne dépassant pas 48 heures. Celles-ci inquiètent son entourage.

Ainsi, pendant quelques semaines, Laurie n'acceptera de manger qu'en présence de sa mère et de son deuxième frère à table.

Ces épisodes mettent en avant l'aspect histrionique et parfois manipulateur de la personnalité de Laurie. 


\section{.Les symptômes d'ESPT chez Laurie}

Lors de certains entretiens, Laurie ressent le besoin de faire le récit de ses cauchemars. L'un d'entre eux sera particulièrement évocateur : «Une jeune fille court dans un bois, elle est poursuivit par un homme [...] J'ai peur, ça pourrait être moi. Après je suis cachée et je vois cette fille se faire violer [...] L'agresseur me voit et se met à me poursuivre [...] Je me retrouve alors dans une salle sombre où deux jeunes enfants sont menacés d'une arme par un autre homme qui leur tire alors dans la tête [...] Quand je me suis réveillée je transpirais, j'avais le cœur qui battait super vite avec un goût amer dans la bouche. »

En plus des flashbacks et cauchemars en rapport avec les violences vécues, elle expliquera avoir quelques fois l'impression d'entendre ou de voir son père ainsi que ses amis décédés, « je suis comme paralysée » «ça m'a déjà fait tomber dans l'escalier du collège». Ces hallucinations peuvent aussi bien avoir lieu au domicile qu'au collège. Elles induisent réactions physiques de stress et sentiment de détresse psychologique. «Je n'en parlais pas, j'avais peur d'être folle», pouvoir exposer ces éléments et les comprendre semble la rassurer, contribuant à leur mise à distance et non à les renforcer comme on aurait pu le craindre face à la personnalité de Laurie.

Elle exprimera ses craintes par rapport à ce qu'elle nomme «mes blocages » à table, devant la télévision ou au collège. Ceux-ci sont en fait des épisodes de dissociation décrits avec précision. Elle les associe à ses difficultés d'apprentissage et de concentration en cours « Je ne suis plus là, j'entends le prof comme s'il était loin et je ne sais plus de quoi il parle », «quand ça m'arrive à table, D (son second frère) s'énerve et dit que je le fais exprès. »

Quelques jours avant un entretien, Laurie croisera son père dans la rue, sur le chemin entre son collège et son domicile. «Pourquoi il est venu par là ? », «je n'arrivais plus à marcher, j'étais terrorisée ! », «je crois qu'il ne m'a pas vu », «après, je me suis imaginée l'insulter dans la rue ». Les trois semaines qui suivront, elle ne pourra sortir seule de son domicile. Sa mère devra la conduire en cours, lorsqu'elle arrive à y aller. Laurie ne fera pas de lien entre le fait d'avoir croisé son père et ses nouvelles difficultés. Même en essayant de le lui suggérer, elle écarte cette possibilité et en est agacée. Ses comportements d'évitement sont là, bien en évidence et Laurie est dans l'incapacité de les identifier. 
Laurie présente une hypervigilance quasi constante, largement majorée la nuit. Elle explique avoir, par exemple, positionné son lit contre le mur, pour pouvoir dormir dos à celui-ci. Elle se dira lassée de ses réactions pluriquotidiennes de sursaut au moindre bruit.

A l'approche d'une chirurgie du genou, sa préoccupation principale est l'anesthésie générale, «je serai coupée du monde», «je ferai plus de cauchemars», «je ne saurai pas ce qui se passe ». Ses craintes seront génératrices d'une anticipation anxieuse négative majeure.

L'irritabilité comme les grands accès de colère se manifestent majoritairement face à sa mère. Celle-ci acceptant tout, mêmes les insultes les plus virulentes sans en tenir rigueur à sa fille.

Les symptômes d'évitement, d'intrusion mais aussi les manifestations neurovégétatives très denses chez Laurie, altèrent son fonctionnement au quotidien. Ils induisent des dysfonctionnements au niveau familial, scolaire et social, générateurs d'une souffrance pour Laurie mais aussi pour son entourage proche.

\section{$\underline{\text { 3. L'entretien à la veille de la confrontation avec le père }}$}

Celui-ci est marqué par l'anxiété de Laurie, qu'elle tentera de masquer par la colère.

Elle pourra décrire la majoration de ses troubles du sommeil, une réactivation de ses troubles du comportement alimentaire ainsi que de l'agressivité verbale face à sa mère.

Au sujet de son père elle s'exprimera avec virulence, «je vais lui cracher à la gueule !», «je vais le défoncer !» mais aussi «je suis certaine qu'il a fait plus », «je sais qu'il s'est passé pire ».

Ses questionnements mais aussi sa culpabilité feront surface, «quand j'étais petite je pensais que c'était normal », «je me suis laissée faire », «j'ai dû le narguer », «c'est forcément de ma faute », «il a peut être subi la même chose lui, quand il était petit ». 


\section{Les multiples prises en charges somatiques de Laurie}

Pendant toute l'année, Laurie aura beaucoup de difficultés à avoir une scolarité régulière. Les motifs seront le plus souvent somatiques, à type de douleurs abdominales, douleurs lombaires, céphalées avec à chaque fois de multiples consultations chez son généraliste ou son pédiatre complétées de nombreux examens.

Elle présentera deux épisodes d'infections cutanées, l'un au niveau d'un piercing qu'elle s'est elle-même fait au niveau du sourcil, l'autre suite à une morsure de son chien à la main.

Le suivi sera interrompu sur deux mois le temps de sa chirurgie du genou. Laurie présente une pathologie méniscale héréditaire nécessitant, comme pour sa mère, une intervention avec par la suite une rééducation. Cette période coïncide avec les vacances d'été.

A la rentrée, Laurie fait la demande de reprendre le suivi. Il n'y aura qu'un entretien au mois de septembre, sa mère nous faisant part d'une nouvelle blessure de Laurie à la cheville nécessitant là encore une intervention, immobilisation puis rééducation.

Quittant le poste au mois d'octobre, je ne fus pas amenée à revoir Laurie.

Ce suivi aura permis à Laurie de trouver une écoute. Un lieu où elle a pu se permettre de livrer sa violence verbale et sa colère sans jugement, allégeant ainsi pour une part, l'agressivité que recevait sa mère au quotidien. Laurie aura aussi identifié certains symptômes, pu y mettre des mots, en avoir des explications, écartant la honte qu'elle y associait.

Enfin, le point central était de l'aider à passer cette période de procédure judiciaire et de confrontation à son père. Laurie sera certes dans la provocation constante, mais n'utilisera pas le temps des entretiens, ses menaces suicidaires pour obtenir une réaction de l'autre. Laurie restera fidèle à son «look de métalleuse » qui deviendra plus modéré. Ses vêtements et son 
maquillage ne seront plus uniformément noirs, son visage dégagé de sa longue mèche; elle se permettra même, à de rares occasions, un authentique sourire.

Les multiples blessures et problèmes somatiques sont les manifestations d'un soulagement qui n'aura été que très partiel. Ils laissent imaginer le long chemin encore à parcourir pour Laurie, vers la résilience.

Je ne sais dans quel délai, mais Laurie a certainement repris un suivi au CAUS, après sa convalescence. Elle avait manifesté lors du tout dernier entretien, l'envie d'intégrer des groupes thérapeutiques animés par des psychologues et infirmiers, chose qu'elle avait jusqu'alors refusé.

\section{$\underline{\text { Bilan de Mlle H. Laurie }}$}

Le cas de Mlle H. Laurie nous permet de constater que la symptomatologie post-traumatique chez l'adolescent est similaire de celle de l'adulte, que ce soit pour l'ESPT que pour les comorbidités psychiques et physiques. Cependant, l'aide médicamenteuse est encore plus complexe que pour le sujet adulte.

Le rôle de soutien des soignants au cours des procédures judiciaires est ici parfaitement évident.

Nous voyons de plus que des mesures médico-sociales existent, dont le maniement doit être maîtrisé par le pédopsychiatre. 


\section{Mlle F.}

Mlle F est une patiente prise en charge à l'hôpital Tenon depuis 2004. En décembre 2012, nous pouvions recenser 10 hospitalisations ainsi qu'un suivi ambulatoire. Ici, j'insisterai sur les deux hospitalisations lors de mes passages en stage dans cette unité, les étés 2010 et 2012.

\section{Hospitalisation d'août 2010 à octobre 2010 (54 jours)}

C'est donc lors de sa sixième hospitalisation dans le service que je suis amenée à prendre en charge Mlle F. Connue de l'équipe, suivie par une psychologue et un psychiatre du service, ils l'adressent cette fois pour une symptomatologie dépressive avec alcoolo-dépendance et mésusage de ses traitements.

Mlle F est la seconde d'une fratrie de trois, une sœur ainée V. et un frère cadet C. Ses parents ont divorcé alors qu'elle n'avait que 11 ans, la mère ayant initialement obtenu la garde des enfants. Son grand-père paternel est décédé mais nous en ignorons la cause. Les antécédents familiaux de la patiente, résumés figure 20, sont peu fournis. Nous comprendrons ce manque d'information de part son histoire de vie.

\section{Figure 20: Génogramme de Mlle F}

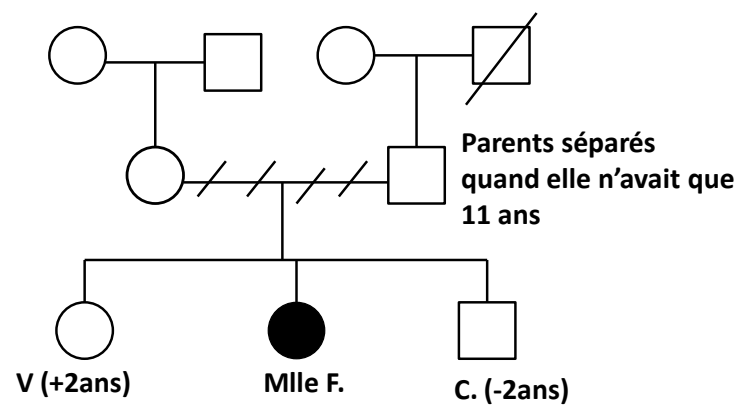

Dans le contexte de séparation de ses parents, Mlle F. sera amenée à consulter une psychologue dès ses 12 ans. C'est à l'âge de 16 ans, qu'elle révélera être victime des viols et attouchements de son frère, plus jeune de deux ans, et ce depuis ses 14 ans. 
Une plainte sera enregistrée, Mlle F est alors placée en foyer à l'ASE (Aide Sociale à l'Enfance) jusqu'à sa majorité avec un administrateur Ad hoc, la mère étant déchue de ses droits parentaux. Son frère sera reconnu coupable avec une injonction thérapeutique mais restera au domicile de la mère. La sœur ainée quant à elle, a quitté le domicile maternel dès sa majorité.

Adolescente, elle a pratiqué le judo à un bon niveau, allant jusqu'aux championnats de France. Elle devra mettre fin à cette pratique suite à une blessure à 16 ans. Elle a obtenu son Bac professionnel dans l'hôtellerie, puis un BTS (Brevet de Technicien Supérieur) de restauration en 2008. Elle travaillera comme chef de cuisine et effectuera même des extras au Crillon entre 2008 et 2010. A 18 ans, elle emménage seule dans un appartement en région parisienne.

Dans ses antécédents médicaux, nous retrouvons: une péritonite à point de départ appendiculaire à 16 ans, une intolérance au lactose et à la cannelle, des migraines et 3 crises d'allure épileptiques depuis 2005 dans des contextes d'alcoolisations massives pour lesquelles elle est suivie en neurologie à Tenon.

Ses antécédents psychiatriques comprennent sa prise en charge débutée à Tenon en 2004. On recense trois tentatives de suicide, par strangulation en 2005 et en 2006 et par intoxication médicamenteuse volontaire en 2008. Elle rapporte des consommations de cannabis entre 17 et 22 ans et toujours actives de tabac (1 paquet par jour) et d'alcool (6 bières par jour ainsi que des alcools forts) le soir, seule au domicile.

Sa prescription médicamenteuse comprend: Paroxétine (DEROXAT®) 20mg, Diazépam (VALIUM®) 30mg, Clonazepam (RIVOTRIL®) 20 gouttes par jour, Amisulpride (SOLIAN®) 100 et Zopiclone (IMOVANE®) au coucher, Hydroxyzine (ATARAX®) 100 si besoin.

Lors de l'entretien d'admission, la présentation de Mlle F. 23 ans, est très masculine. Cheveux bruns courts et vêtements de sport, cherchant probablement à masquer son surpoids. Elle se montre introvertie et mal à l'aise, fuyant le regard. Elle s'exprime sur un ton monocorde, le visage ne laissant transparaître aucune émotion. Cependant, elle se présente comme bisexuelle, se comparant à sa sœur qu'elle dit homosexuelle. 
Cliniquement, nous constatons ce qu'il semble être une symptomatologie anxio-dépressive avec tristesse, ralentissement psychomoteur, anhédonie, mais sans idée de mort. Elle décrit des éléments d'ESPT, flash-back, cauchemars, hypervigilance et conduites d'évitement, très invalidants notamment au niveau professionnel. Elle a d'ailleurs été récemment licenciée pour absentéisme. En plus de ses alcoolisations massives et ses mésusages de traitements qu'elle banalise, elle décrit des comportements d'automutilations à type de scarifications, accumule les conduites à risque et de revictimisation. Elle aurait reçu plusieurs menaces de dealers mais aussi laissé des individus «peu recommandables » loger dans son appartement.

Les échelles d'auto-évaluation proposées à l'arrivée montrent un IES-R (Impact of Event Scale-Revised) à 77 et une HAD (Hospital Anxiety and depression) avec 16/21 pour l'anxiété et $15 / 21$ pour la dépression.

Déjà en confiance avec l'équipe soignante, l'hospitalisation lui permettra de revenir sur sa relation avec ses parents. L'attitude ambigüe et culpabilisante lors du procès, notamment de sa mère qui lui reproche sa plainte, allant même jusqu'à soutenir C. Son sentiment de «mise à l'écart » du cadre familial, C. vivant encore chez la mère et n'ayant d'ailleurs pas respecté l'injonction thérapeutique. Concernant le père, elle dit avoir toujours gardé une assez bonne relation avec des contacts réguliers. Mlle F. décrit sa sœur comme peu soutenante et n'aurait quasiment plus de liens avec ses grands-parents maternels.

Elle révélera en psychothérapie avoir été de nouveau victime d'un viol, au mois de février 2010, cette fois au domicile, par l'un de ses «squatteurs ». Elle se refuse à porter plainte de peur de représailles. D'ailleurs, lors d'une permission, elle affirmera avoir été menacée de mort par arme blanche par une connaissance de son agresseur. Evènement qui la décidera dans la démarche de plainte contre X. Deux agents de police se déplaceront à l'hôpital afin d'enregistrer sa déposition.

Par ailleurs, ses addictions sont manifestes : tabac, alcool, cannabis mais aussi les traitements médicamenteux qu'elle consomme de façon totalement anarchique dans un but de sédation. En dehors de l'antidépresseur, elle peut sur une journée multiplier par plus de trois les doses des autres molécules. Elle justifie cette automédication, sans la critiquer, par ses reviviscences qu'elle décrit d'une rare intensité. En effet, dans le service, celles-ci peuvent la conduire à frapper dans les murs de sa chambre, occasionnant même lors d'une crise, une fracture diaphysaire du troisième métacarpien de la main droite. De plus, elle présentera plusieurs 
crises d'allure épileptique, occasionnant des chutes, avec pour conséquences des entorses au poignet gauche, à la cheville droite et aux cervicales. Compte tenu de ses antécédents d'épilepsie, un avis spécialisé sera demandé et des investigations neurologiques réalisées. Un traitement anti-comitial sera prescrit, bien que ces manifestations semblent plus de l'ordre de la conversion. Mlle F. se retrouve alors boitant dans les couloirs, portant une minerve, une attelle à chaque poignet ainsi qu'à la cheville et devient l'objet de l'attention des autres patients du service.

Le reste du bilan somatique réalisé mettra en évidence une infection sexuellement transmissible récente à papillomavirus, que l'on peut supposer secondaire à sa dernière agression sexuelle, nécessitant un suivi régulier à vie.

Les modifications des traitements médicamenteux aboutiront à l'ordonnance suivante: Venlafaxine LP (EFFEXOR®)375mg, Miansérine (ATHYMIL®) 60mg, Alimémézine (THERALENE®) 20mg au coucher, Alprazolam (XANAX®) 0,5mg 3 fois par jour, Lévétiracétam (KEPPRA®) 2000mg.

Un terme sera mis à l'hospitalisation après plus d'un mois et demi, Mlle H étant embauchée dans les cuisines d'un restaurant parisien après avoir passé plusieurs entretiens lors de ses permissions, cependant, la symptomatologie n'est que partiellement améliorée. Cette longue hospitalisation, comprenant un temps de sevrage de l'alcool, aura surtout permis une adaptation des traitements et de leurs dosages. Ses troubles majeurs du sommeil seront eux peu améliorés. Cependant, l'auto-évaluation permet de trouver à la sortie une HAD avec 10/21 pour l'anxiété et 9/21 pour la dépression.

\section{L'année 2011}

En 2011, Mlle F sera hospitalisée à trois reprises pour un total de 129 jours. Admissions rythmées par une procédure judiciaire à laquelle elle sera de nouveau confrontée, en lien avec le dernier viol au domicile. Notons une nouvelle intoxication médicamenteuse volontaire au mois d'avril dans le cabinet de son avocate mais aussi la reprise de ses consommations abusives d'alcool et de médicaments. Cette période sera marquée par une incapacité à retravailler, des demandes de congés longue maladie et d'Allocation Adulte Handicapé seront effectuées. 2011 sera aussi l'année du diagnostic de son diabète de type 2. 
Au cours de l'été 2012, Mlle F. est de nouveau adressée pour mésusage de ses traitements, cette fois par la psychologue ayant constaté lors de ses entretiens hebdomadaires, un état de sédation important.

La présentation de Mlle F est identique, figée avec de nouveaux kilos acquis, en témoigne un indice de masse corporelle à 30 .

Depuis la précédente hospitalisation de 2011, il y a 5 mois, elle a maintenu l'abstinence en alcool et cannabis, largement remplacée par les médicaments. En effet, cette fois, elle ne nie pas ses consommations excessives de benzodiazépines et de Cyamémazine (TERCIAN ®) et en perçoit le handicap. Il semblerait que Mlle F. ait mis à distance ses conduites à risque, elle ne présente plus d'épisode de crise aussi bien clastique que pseudo épileptique. Une relative maîtrise de ses reviviscences et cauchemars a pu être acquise, notamment par les séances de sophrologie et de relaxation proposées par l'équipe soignante.

Elle vit toujours dans ce même appartement mais ne travaille plus depuis début 2011. Elle explique avoir rapidement démissionné du restaurant parisien, s'étant rendue compte du non respect des règles d'hygiène et de sécurité de l'établissement. Pour Mlle F., le travail constituait un élément stable d'étayage. Nous ne pouvons alors que constater une dégradation progressive de la patiente avec un repli au domicile et une évolution quasi déficitaire. En effet, dans le service, elle se montrera d'une grande passivité avec des attitudes régressives.

Cependant, cette hospitalisation sera le temps d'avancées en psychothérapie. Elle revient sur des détails de son enfance, avant le divorce de ses parents, et décrit alors une mère peu présente et très investie dans des activités extra-familliales. Une juste distance pourra être prise avec celle-ci : «je n'attends plus de reconnaissance de sa part ». Elle en pointe d'ailleurs les ambivalences du comportement, relatant ses visites qui ne peuvent se faire que si sa mère trouve un alibi pour que C. soit absent. Elle en critique sans culpabilité l'attitude protectrice auprès du frère. 
Pour la première fois depuis 8 ans, elle se permettra de parler plus en détails de son père. Elle révélera alors le passage d'un statut de parent protecteur à celui d'agresseur incestueux. La mère souvent absente, c'est lui qui s'occupait d'elle petite, il l'accompagnait dans ses activités extra-scolaires et compétitions de judo. Or, c'est justement à l'occasion de ces déplacements que se déroulaient les agressions. Pour tenter d'y mettre fin, elle dit s'être volontairement blessée pendant une compétition. Occasionnant une cervicopathie, elle devenait inapte à la pratique du judo. Elle aurait été victime d'inceste par son père de 6 à 20 ans, les agressions auraient donc continué après le divorce mais aussi pendant son suivi à Tenon sans jamais qu'elle n'en fasse référence.

Sur le plan somatique, son suivi en neurologie a été maintenu. En gynécologie, une colposcopie a du être réalisée devant des frottis suspects. Ses douleurs cervico-lombaires, largement majorées par son surpoids, nécessiteront un avis de l'équipe antidouleur avec la décision d'introduire de la Prégabaline (LYRICA®). Le diabète a continué de progresser, le suivi par son généraliste ne suffisant plus, elle est dirigée en endocrinologie où sera posée l'indication d'antidiabétiques oraux.

Une nouvelle fois les traitements médicamenteux sont modifiés et bien que restant importantes les doses sont revues à la baisse. Son ordonnance comprend alors : Venlafaxine 300mg, Aripiprazole (ABILIFY ®) 10mg, Hydroxyzine (ATARAX®) 100mg , mais aussi, Lévétiracétam (KEPPRA®) $2 \mathrm{~g}$ et Metformine 500mg.

Cette hospitalisation de deux mois et demi sera surtout le temps de nouvelles révélations et d'un travail d'élaboration avec la psychologue mais aussi de discussion d'équipe afin d'envisager des outils d'étayage pour Mlle F. Etant dans l'incapacité de travailler, un projet en hôpital de jour pluri-hebdomadaire sera établi avec, en attendant l'acceptation de son dossier, une convalescence de plusieurs mois. 


\section{Le bilan de Mlle F.}

Auprès de soignants à son écoute, elle a pu un temps pallier un soutien familial totalement défaillant, culpabilisant, voire même délétère. Ayant fait preuve de maturité et d'autonomie par le passé, menant de front procédures judiciaires, études, projets professionnels, Mlle F. se retrouve en 2012 dans une position régressive alors même qu'une distance a pu être prise avec sa mère et plus récemment avec son père.

Sa position régressive et son apragmatisme progressif, bien qu'ayant 25 ans, pourraient être proches de ce que R. Spitz a décrit chez les bébés en carence affective, comme étant «la dépression anaclitique » voir «l'hospitalisme ».

Mlle F. nous met aussi face à un relatif échec sur le plan médicamenteux. Nous voyons bien une escalade difficilement maîtrisable conduisant à des ordonnances vertigineuses sans réel apaisement des symptômes. Une juste utilisation doit donc être recommandée dès le début des suivis avec une vigilance accrue quant aux dérives possibles chez des patients souvent sujets aux addictions.

Les durées d'hospitalisations conséquentes (entre celle de Tenon et sa convalescence, elle aura eu une prise en charge hospitalière temps plein de 8 mois en 2012), ses traitements et soins somatiques, nous permettent, encore mieux que les cas précédents, d'illustrer l'ampleur des dépenses de santé chez ces patients. Dépenses qui, au final, ne sont pas uniquement psychiatriques ou même de santé, mais aussi liées à une perte de productivité entraînant un coût sociétal indirect conséquent. 


\section{Mlle R.}

Mlle R est aussi une patiente ayant bénéficié de soins en hospitalisation à Tenon au cours de l'été 2010, avec deux prises en charge très rapprochées, menant à quasiment 2 mois d'hospitalisation.

\section{$\underline{\text { Hospitalisation du 16/07/2010 au 30/08/2010 }}$}

Mlle R. est âgée de 17 ans et 10 mois lors de cette première admission dans le service pendant les vacances scolaires d'été.

Elle a été dirigée vers la consultation de Tenon par sa psychologue scolaire, suite à des dessins faits en classe, alertant l'équipe pédagogique. A cette psychologue, elle a révélé avoir été victime à deux reprises, à 6 puis 11 ans, d'agressions sexuelles par le fils d'amis de ses parents, celui-ci ayant 6 ans de plus qu'elle.

Le psychiatre consulté à Tenon, a posé l'indication d'une hospitalisation, devant la symptomatologie d'ESPT invalidante et les menaces suicidaires exprimées en entretien.

Mlle R., encore mineure pour quelques semaines, se présente dans le service, accompagnée de son père. Nous sommes frappés par la présentation extrêmement masculine de la patiente. Une carrure de basketteur, des cheveux bruns attachés à la nuque, elle porte un maillot de basket avec un survêtement de sport et des baskets aux pieds. Elle ne laisse transparaître aucun indice de féminité.

L'entretien d'admission se fera dans un premier temps avec le père afin de recueillir les informations et antécédents généraux, puis seule avec Mlle R., toujours en présence d'un infirmier. Mlle R. s'est alors positionnée dans un coin de la pièce, de façon à ne faire face à aucun des intervenants. Elle laissera très majoritairement son père répondre aux questions, fixant le sol.

Avec les antécédents familiaux, nous obtenons le génogramme figure 21. 


\section{Figure 21: Génogramme de Mlle R.}

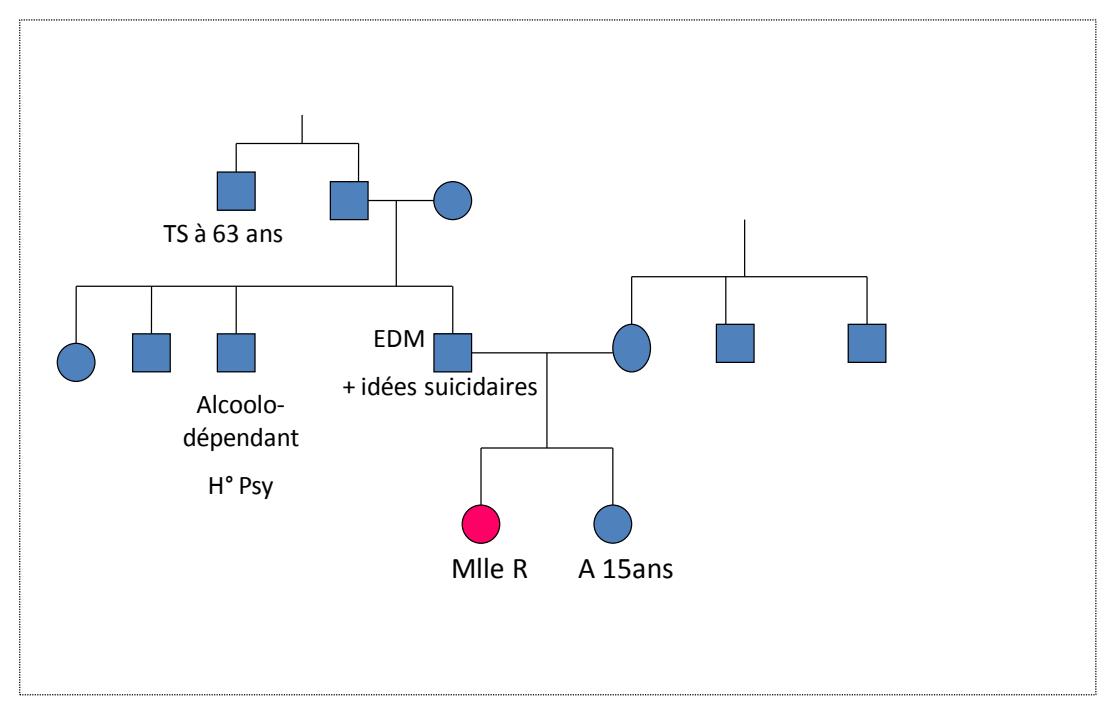

Nous apprenons alors que Mlle R. est l'ainée d'une fratrie de deux. Sa sœur cadette A. est âgée de 15 ans. La famille paternelle est d'origine italienne. Elle pratique, comme nous avions pu le deviner, le basket à un niveau national, avec des entraînements pluri-hebdomadaires soutenues. Elle est lycéenne en première scientifique. Elle devra redoubler son année, ayant comme projet de changer de filière, espérant prendre une orientation STL (Sciences et Technologies de Laboratoire). Elle souhaiterait de plus, quitter le domicile parental pour s'installer à Lyon, où vit une tante paternelle.

Nous retrouvons un grand oncle paternel ayant fait à 63 ans une tentative de suicide et un oncle alcoolo-dépendant ayant été hospitalisé à maintes reprises en psychiatrie. Les antécédents concernant le père ne nous seront communiqués que dans un second temps, et au cours de circonstances particulières que nous aborderons plus tard.

Après les informations de base de l'anamnèse rapportées par le père, celui-ci nous présente sa fille comme «absente et déconnectée » à des moments selon lui incongrus de la journée. Ces phases ayant alors des conséquences sur sa scolarité et la pratique de son sport. Il décrit de grands accès de colère pouvant aller jusqu'à la violence physique. Il note deux épisodes majeurs, l'un contre lui et l'autre face à son entraîneur à qui elle reprochait «d'être restée sur le banc». Il met ses «coups de sang » sur le compte de ses origines italiennes. En dehors de ses nombreuses heures d'entraînement, elle pratique bien d'autres sports qui selon lui ont un rôle « d'hyper activité compensatrice pour éviter de penser ». 
Fixant toujours le sol et bougeant nerveusement les jambes, Mlle R. ponctue le récit de son père de quelques éclats de voix et de rire, largement défensifs. Comme déstabilisé par la réaction de sa fille mais voulant nous prouver une complicité entre eux, il fait de même.

Avec Mlle R. seule, nous devrons nous contenter, lorsqu'elle accepte de nous les donner, de réponses laconiques. Elle nous dira ne pas consommer de toxiques et évoquera ses troubles du sommeil essentiellement en lien avec des cauchemars. Elle reste réticente à aborder les autres troubles évoqués auparavant par son père et mutique quand il s'agira des idées suicidaires rapportées en consultations.

Mlle R. semble accepter cette hospitalisation passivement et contrainte par ses parents, sans en percevoir l'objectif.

$\mathrm{Au}$ cours de cette hospitalisation, plusieurs entretiens seront réalisés avec les parents et la sœur. Ils permettront de comprendre le fonctionnement familial et leur difficulté de communication, ainsi les questions de fond ne pourront jamais être abordées. Mlle R. adoptant une position enfantine en présence de sa sœur, les deux chuchotant et ricanant sans relâche. A noter que la cadette a emprunté le style vestimentaire et l'attitude masculine de sa sœur ainée. La mère, banalise les symptômes de sa fille, optant pour un ton défensif mais néanmoins agressif permanent. Même lors de simples visites, chacun reste dans un coin de la chambre; les échanges, même verbaux, se font rares.

Comme bon nombre de patients du service, Mlle $\mathrm{R}$ passera beaucoup de temps à écrire et dessiner. Elle nous fera parvenir quelques documents.

Ci-dessous, deux d'entre eux (document 3 et 4) où Mlle R exprime sans détour ses difficultés. 


\section{Document 3}

$\Rightarrow$ Yeux être nec:

$\rightarrow$ Y'aime encore Kévin:

$\rightarrow$ Ye sais pus oci jén suis avee Quentin. Ye sais flus xi je l'aime encore.

$\rightarrow$ Ye veux pas dormir, j'ai puer de dormir parce que je fais des canche mares. Ife

$\rightarrow$ K' crois ples en l'anour.

$\rightarrow$ Ke crois que j'aime plus les neces.

$\rightarrow$ Ye veux pas grandir.

$\rightarrow$ Ye veax fers avanuer. C'est trop dare. j'en ai marre de me battre.

$\rightarrow$ Y'en ai narre de vivke. La sert a kien que je vive. Ye suis un poids

pour toun cents gui n'entourre.

$\rightarrow$ Ye veux pless uivre car c'est trop dere. Y'en ai narre de souffir at de feire souffir mes parents.

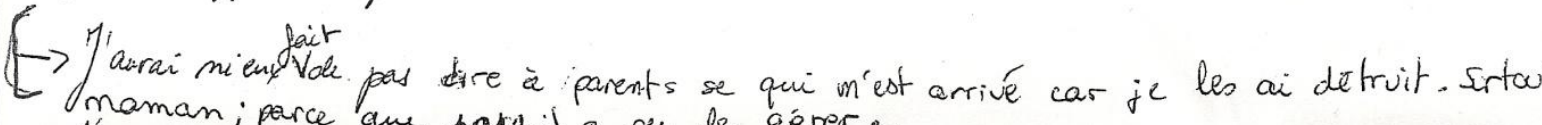
$\rightarrow$ Y'en ai narre de ma nere parce qu' cele peit pas l'effort de me comprendre mair d'an coté je leur parle pas.

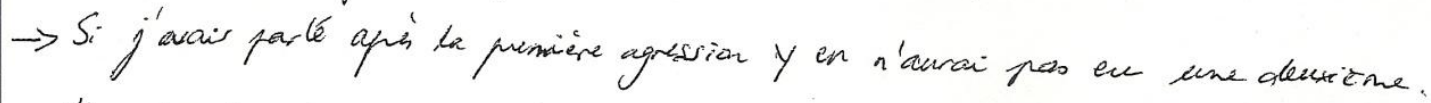
$\rightarrow$ H'en wan à mes parents de pas avoir an paroir m'aider quand j'en wovis vrainent besoin, je dis pas qu'anjourd'hui j'en ai pas hesoir, it pais de tonte fagan $/$ je veux pess qu'ib aident, per hout manen.

$\rightarrow$ Ya der moments. noments ouj jeme dent aube, invisible; pourtant je bais que je le suis pas.

$\rightarrow$ Je rigdest puer un sienniome quand ga ca pas, fue cest pas marrant.

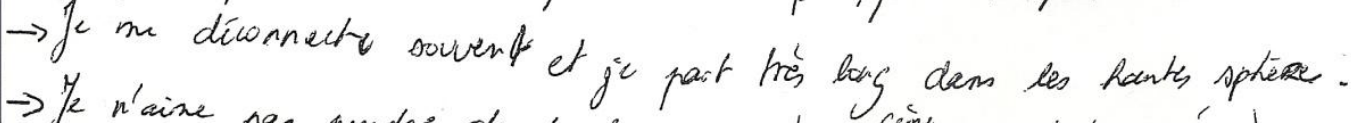
$\rightarrow$ Ye n'aine pas puerere de douche paree qu'en Ginu ga e'est pasé dam sa salle de $\rightarrow$ Mes humeurs sont comse des montagus ueases.

$\rightarrow Y^{\prime}$ arrive pas à parler car j'ai des bocegeas.

$\rightarrow$ I'ai de blocajes dés qu'on tache de pies an de lain ia a qui m'est arrivén. $\Rightarrow y^{\prime}$ ai l'impression are i..... . . . . . ... imilot ane ic 
Document 4

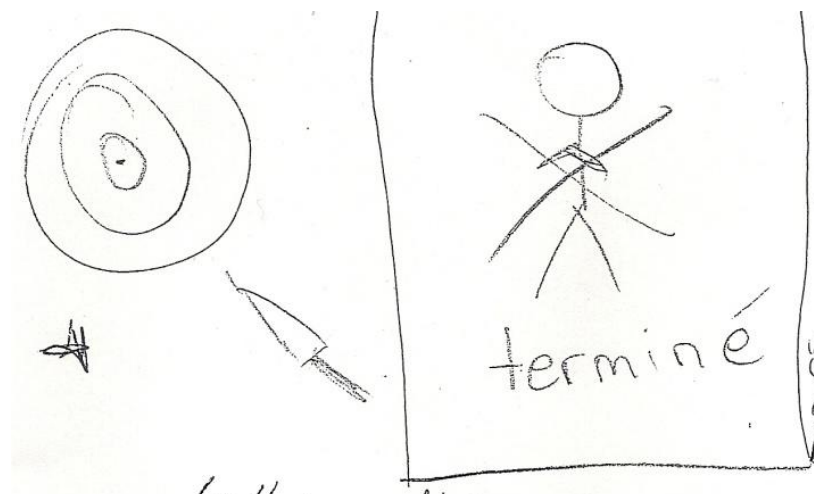

$\Rightarrow$ Lettre adien

$\rightarrow$ voir Mord:

$\rightarrow$ sentiments sur actes.

$\rightarrow$ desin

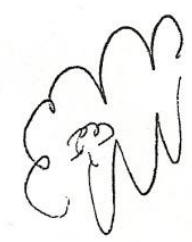

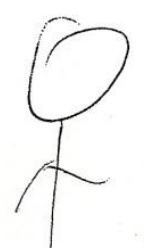

en meme
tenme il we
terissait des
truc er

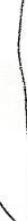

ulne el continas. cia siarrétea

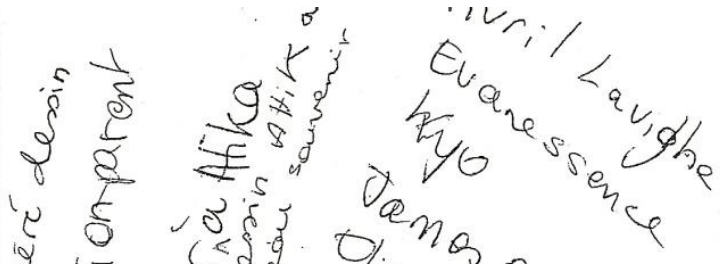

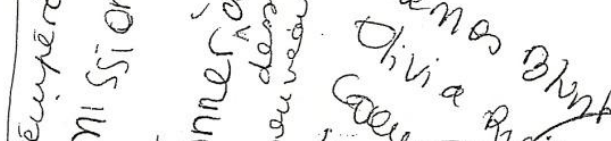
( )
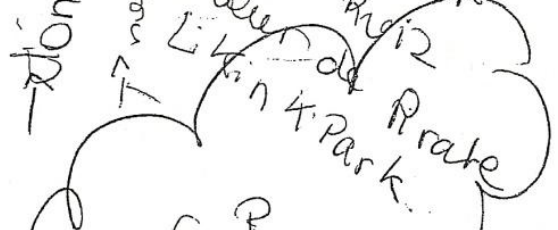
douvenuos a dit stop. il a continué

jeu de le faire dans liau f: essayer dans le grage fait dano la chambre de ses la chantre de ses la chambre de son pere la bureau de son pere nak moit Itamélie paner teluc avee Ané lie dle mon s elle partis. parceque be hamak do cossa.

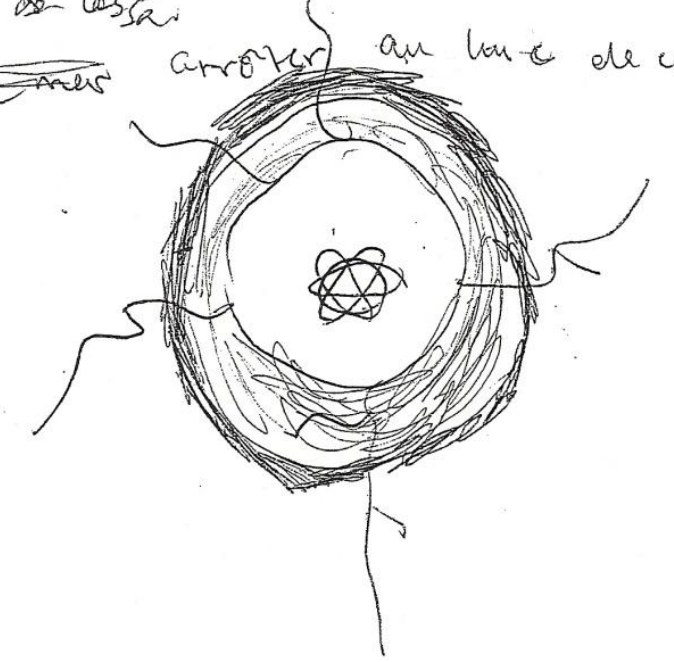

157 
En plus de ce qu'elle peut nous communiquer par ses écrits, nous pouvons aussi constater les symptômes manifestes d'ESPT. En effet, lors des entretiens quotidiens, elle présentera des moments de dissociation pouvant être assez longs. Elle s'autorisera à y mettre quelques mots, cette accroche nous permettant alors de lui en expliquer les mécanismes, ce qui semble dans ce domaine l'apaiser. Elle nous fait de plus part d'un épisode de reviviscence qu'elle décrit comme quasi hallucinatoire, l'agresseur présent dans sa chambre le visage au dessus d'elle.

A l'opposé, son hypervigilance constante, ses troubles du sommeil, faits de lutte contre l'endormissement et de cauchemars, mais aussi son agitation anxieuse en début de soirée, difficilement contenue et indifférente à la réassurance, resteront inabordables. Nous devrons pour ces manifestations, faire face à son extrême réticence voire son mutisme d'où une impossibilité d'élaboration.

Tout comme à l'écrit, elle nous fera part de son inquiétude pour sa petite sœur. Mlle $\mathrm{R}$ s'interroge sur la possibilité pour sa cadette d'avoir été victime de ce même jeune homme. Elle parvient aussi à exprimer ses difficultés à vivre au domicile familial, lieu où se serait déroulée l'une des agressions, justifiant alors son projet de déménagement à Lyon.

Elle manifeste alors une grande ambivalence vis-à-vis de sa famille, prise entre le désir de s'éloigner du domicile et son manque de maturité et d'autonomie, l'empêchant de mener à bien son projet. Elle critique de plus « la pression que lui met sa mère pour porter plainte ».

Sur le plan médicamenteux, de l'Escitalopram (SEROPLEX ${ }^{\circledR}$ ) est introduit avec un essai de Divalproate de sodium (DEPAKOTE®), ainsi que différents hypnotiques et anxiolytiques sans amélioration notable. L'évaluation de l'efficacité sera d'autant plus compliquée que la patiente en a une très mauvaise observance, feignant d'ingérer les comprimés.

A ce stade de l'hospitalisation et après un mois, aucune avancée majeure n'a été opérée, mais une entrée dans le soin ébauchée. En accord avec les parents, le terme de l'hospitalisation est alors décidé, afin de poursuivre les soins en ambulatoire. Cette perspective de sortie marquera un bouleversement dans la prise en charge. 
Mlle R. rompra tout dialogue. Déjà peu loquace, elle en deviendra mutique et multipliera les passages à l'acte. Elle échappera à plusieurs reprises à la vigilance de l'équipe, fuguant du service, mais sera rapidement retrouvée dans une rue non loin ou dans une zone en chantier de l'hôpital. Elle en ramènera clous rouillés ou tessons de bouteille avec lesquels elle se scarifiera les avant bras, les poignets, le ventre et les cuisses.

Adoptant une attitude de franche opposition, dans le refus des entretiens, elle en viendra à utiliser les meubles de sa chambre pour bloquer sa porte. Elle se contentera alors de lâcher avec une extrême violence, ses idées et scénarii suicidaires entre autre de pendaison.

Les entretiens familiaux se multiplieront mais seront peu aidants, ses parents comprenant difficilement ce basculement, disqualifiant les soins, «puisque notre fille semble aller plus mal » et n'identifiant pas la difficulté de Mlle R. à vivre sur le lieu de ses agressions.

L'un de ces entretiens marquera un changement de position des parents. En effet, Mlle R. leur fera part sans détour, pour la première fois, de ses idées de mort. Un lourd silence suivra cette annonce puis la mère, sortant de sa froideur, laissera transparaître un regard inquiet pour sa fille « je ne savais pas que tu pensais à ça ! ». Le père, lui, en larmes et se lance sans retenue, dans le récit de ses propres scénarii suicidaires 2 ans auparavant. Il détaille ses difficultés professionnelles l'ayant conduit à un projet de précipitation depuis un pont, passage à l'acte détourné par un appel de sa femme. Eléments dont aucun membre de la famille n'avait eu connaissance.

L'escalade de mise en danger se poursuivant, la sortie d'hospitalisation devra être de nombreuses fois reportée. Compte tenu de notre cadre d'hospitalisation (service ouvert et uniquement des hospitalisations libres), nous nous retrouvions dans l'incapacité de la protéger. La décision en accord avec ses parents est prise de la transférer sous contrainte sur son secteur. Nous apprendrons plus tard qu'elle y restera trois jours. 
Très vite sortie de son secteur, Mlle R. a arrêté tous les traitements médicamenteux mais a repris le suivi ambulatoire avec sa psychologue scolaire et le psychiatre de Tenon.

Devant la persistance d'idées suicidaires et probablement sans tenir compte du déroulement de l'hospitalisation précédente, celui-ci nous l'adresse de nouveau pour ce motif.

Lors de cette hospitalisation, Mlle R. est majeure. Elle nous explique vivre encore chez ses parents et avoir pu faire sa rentrée dans un lycée du $13^{\text {ème }}$ arrondissement de Paris en STL. Avec ses parents, ils auraient un projet de location d'appartement pour elle, non loin du Lycée pour pallier les longs trajets en train. Les difficultés au domicile se seraient majorées, Mlle R devant travailler dans les transports devant l'impossibilité de lâcher sa vigilance au domicile pour se concentrer sur son apprentissage scolaire.

L'alliance thérapeutique avec l'équipe d'hospitalisation est encore très fragile et de nouveau nous sentons rapidement s'amorcer une nouvelle escalade de mise en danger. Mlle R. nous affichant des dessins provocants et laissant bien en vue des mots explicitant des idées suicidaires mais refusant les entretiens avec les psychologues et les psychiatres du service.

Un terme est mis à cette hospitalisation en accord avec la patiente et ses parents afin de poursuivre les soins ambulatoires probablement mieux adaptés à Mlle R.

Il est important de noter que la première hospitalisation de Mlle R a été compliquée par les changements d'intervenants, aussi bien psychologues que psychiatres, dues aux vacances d'été de chacun. Ceci n'aura facilité en rien l'alliance thérapeutique chez cette patiente déjà difficilement en confiance. De plus, de par le déroulement de la première hospitalisation, l'indication de la seconde est discutable puisque de manière prévisible, et dès son arrivée, sa présentation et son fonctionnement seront les mêmes. 


\section{$\underline{\text { Bilan de Mlle R. }}$}

Ce cas clinique nous permet de percevoir l'importance de l'évaluation des patients.

Le travail fait en amont de l'hospitalisation libre, afin de s'assurer de l'investissement dans les soins et d'autant plus, chez cette adolescente se structurant sur une personnalité limite.

Nous devons aussi être capables de reconnaître les limites des structures spécifiques du psychotraumatisme afin de pouvoir être garants de la sécurité des patients et de soins adaptés, et donc en cela, savoir rediriger vers des institutions plus adaptées, même temporairement.

Cette situation d'impasse thérapeutique nous conduit à aborder les notions de «burn out » et de « traumatisme vicariant ».

Le terme de «traumatisme vicariant» a été introduit en 1995 par Pearlman et Saakvitne [175]. Il s'agit d'une « usure de compassion » consécutive au contact répété avec des victimes traumatisées. Il « relève de l'accident de travail pour ceux qui travaillent avec des victimes et il ne reflète ni la pathologie du thérapeute ni l'intentionnalité de la victime » (Pearlman 1996) [176]

Lors des hospitalisations de Mlle R., nous avons pu constater l'impact des récits et de la souffrance de cette adolescente sur les membres de l'équipe. Pour certains, nous notions une difficile prise de distance face à cette histoire de vie, un sentiment d'impuissance et d'épuisement, confrontés à ses menaces suicidaires et multiples passages à l'acte.

Là encore, nous ne pouvons que souligner le caractère indispensable d'un travail coordonné en équipe pour les soins de patients traumatisés. Il faut de plus savoir passer la main temporairement, pour permettre à un thérapeute ou à une équipe de soins épuisés par une situation difficile, de se restructurer. 


\section{CONCLUSION DES VIGNETTES CLINIQUES}

Ces quatre cas cliniques ont été choisis pour leur caractère d'exemple. Chacun à sa façon, illustre un point crucial de la psychopathologie, ainsi que les comorbidités retrouvées chez des victimes de violences sexuelles dans l'enfance.

Ces patientes ont pour point commun des symptômes d'ESPT invalidants, mais elles ont aussi leurs particularités :

-Mlle L., nous interpelle avec sa symptomatologie dépressive, ses douleurs de fibromyalgie et nous montre l'importance du soutien familial.

-Mlle H., expose la clinique chez l'adolescent et l'évolution d'une personnalité vers un trouble borderline.

-Mlle F., de par ses multiples comorbités nous laisse imaginer le coût vertigineux de ses nombreuses prises en charge.

-Mlle R., permet de mettre l'accent sur les difficultés et la souffrance des soignants face à de telles histoires de vie.

De plus, elles nous poussent à une réflexion sur nos pratiques et à en accepter les limites. L'hospitalisation ne devant par exemple pas être au centre de la prise en charge, mais comme les traitements médicamenteux, doit être utilisée à bon escient, au risque d'être délétère sur le plan social ou professionnel.

En élargissant notre champ d'approche, nous percevons le grand risque de précarité et d'exclusion sociale de nos patients, justifiant alors les compétences des associations de victimes : information, soutien social, aide dans les démarches juridiques mais aussi savoir diriger vers les soins. 
Ces présentations nous conduisent à porter notre attention sur l'environnement familial des victimes, que ce soit à la suite des agressions ou sur les générations précédentes. En effet, les quatre jeunes femmes sont issues de familles que l'on peut qualifier de dysfonctionnelles. S'y manifestent alors un défaut de soutien, des carences affectives et peuvent même se révéler être le lieu des agressions. Une des clefs de la prévention des violences sexuelles sur les mineurs ne serait elle pas de se pencher sur ces familles en difficultés?

Comme nous pouvons le supposer au travers des vignettes cliniques, mais aussi comme nous l'avons vu précédemment dans «les caractéristiques des agresseurs sexuels sur mineurs », avoir été victime de violences sexuelles dans l'enfance peut potentiellement prédisposer à devenir soi même agresseur. Quand est-il alors des dépenses liées aux suivis médical, social et judiciaire de ces sujets devenus agresseurs sexuels ? 


\section{CONCLUSION}

Le travail ici réalisé, permet un tour d'horizon de la question des violences sexuelles dans l'enfance et leurs conséquences à l'âge adulte, mais pas uniquement d'un point de vue médical. Nous sommes partis de généralités historiques et épidémiologiques, pour arriver à quatre cas particuliers sous forme de vignettes cliniques.

En France, jusqu'à 10\% des femmes et 5\% des hommes pourraient avoir été victimes de violences sexuelles dans leur enfance [129,23]. L'inceste concernerait deux millions de français [7]. Il semble alors incontournable de dépasser les divergences entre définitions juridiques et médico-sociales, pour s'accorder face à leurs terribles impacts individuels et sociétaux.

Les violences sexuelles sur mineurs, trop souvent passées sous silence dans notre société, restent encore largement sous évaluées notamment par le corps médical. Le soignant est pourtant, quelle que soit sa spécialité, en première ligne dans son repérage. La nécessité de rechercher de tels antécédents chez nos patients, mêmes adultes, ne doit plus poser question et devrait être enseignée au cours de notre formation. N'oublions pas que le soignant peut s'affranchir du secret professionnel afin d'informer «les autorités judiciaires, médicales ou administratives de privations ou de sévices, y compris lorsqu'il s'agit d'atteintes ou mutilations sexuelles, dont il a eu connaissance et qui ont été infligées à un mineur ou à une personne qui n'est pas en mesure de se protéger en raison de son âge ou de son incapacité physique ou psychique » (article 226-14 du code pénal). Ces situations particulières font partie des dérogations facultatives au secret médical.

Les adultes victimes de violences sexuelles dans l'enfance sont des patients d'une grande complexité. En effet, comme nous avons pu le voir grâce à nos quatre cas cliniques, il n'y a pas qu'une unique présentation psychopathologique au décours de traumatismes sexuels, mais une multitude de manifestations handicapantes et altérant la qualité de vie. L'Etat de Stress Post Traumatique est une notion spécifique, intégrée aux classifications internationales, qui a le mérite d'exister. Cependant, les troubles post-traumatiques vont bien au-delà de cette seule entité. En effet, assez classiquement, nous observons des troubles du comportement alimentaire ainsi que des comportements auto-agressifs s'intégrant à un trouble de la personnalité ou encore à un épisode dépressif majeur, mais les séquelles post-traumatiques peuvent aussi être d'ordre somatique, se devant alors d'être connues et non négligées. 
Les troubles présentés sont sensibles à une prise en charge de qualité, en témoignent nos vignettes cliniques. Ces soins spécifiques, mais sans modèle type, comprennent des psychothérapies se voulant adaptées à chaque patient, des thérapeutiques médicamenteuses symptomatiques à manier avec prudence, sans oublier les aspects sociaux, juridiques et relationnels. Dans ce sens, des consensus publiés en France par l'HAS (Haute Autorité de Santé), telles que «Repérage et signalement de l'inceste par les médecins : reconnaitre les maltraitances sexuelles intrafamiliales chez le mineur» [117] ou encore «Affection psychiatrique de longue durée: Troubles anxieux graves»[116], peuvent guider notre pratique médicale. Repérer, aider et soigner ces patients nécessitent donc de nombreux intervenants, tous indispensables: médecins traitants, scolaires ou du travail, médecins légistes, psychiatres spécialisés en psychotraumatologie mais aussi psychiatres de secteur, psychologues, travailleurs sociaux, éducateurs ainsi que les associations de victimes. Le travail en réseau est alors incontournable. De tels réseaux organisés existent en France et fonctionnent dans des grandes villes comme Lille ou Paris. Il en faudrait bien d'autres, en regard des demandes croissantes et à mesure que se lève le tabou du traumatisme entre autre secondaire aux violences sexuelles.

Nous ne pouvons alors échapper à la question du coût de ces soins spécifiques ou non. Notre étude a donc voulu évaluer la faisabilité d'une quantification des dépenses de santé de ces victimes. En utilisant un échantillon de sujets adultes suivis à Tenon, ayant été victimes de violences sexuelles avant l'âge de 15 ans, nous avons pu conclure à une majoration significative de leur Consommation Médicale Totale par rapport à celle de la population générale française en 2012. La nécessité d'études à plus grande échelle paraît alors indiscutable afin d'obtenir une quantification optimale des coûts, mais aussi de pouvoir calculer les dépenses indirectes des domaines sociaux, juridiques et professionnels. Ainsi, les résultats justifieraient auprès des autorités les ressources mobilisées et même permettre une optimisation de leurs allocations.

Nous pouvons conclure de ce travail, que les violences sexuelles engendrent un coût individuel psychique pour la victime, financier pour la société mais aussi un coût émotionnel pour l'entourage ainsi que pour les intervenants participant au soutien médical et social. Elles se doivent donc d'être inscrites dans une préoccupation de santé publique. Fournir des moyens humains, en favorisant l'information et la formation des professionnels, pourraient permettre de mieux dépister voir d'anticiper les troubles secondaires coûteux et idéalement de prévenir les violences sexuelles sur mineurs. 


\section{BIBLIOGRAPHIE}


1. Agence France Presse «Les violences sexuelles sur mineurs bondissent de $11 \%$ » Libération. Septembre 2012. Consultable sur: http://www.liberation.fr/societe/2012/09/28/les-violencessexuelles-sur-mineurs-bondissent-de-11 849600

2. Alexander P.C., Anderson C.L. et al. "Adult attachment and longterm effects in survivors of incest." Child Abuse \& Neglect. 1998; 22(1) : 45-61.

3. Allers CT, Benjack KJ, White J, Rousey JT. "HIV vulnerability and the adult survivor of childhood sexual abuse.” Child Abuse Negl. 1993;17:291-298.

4. Alonso, J., Angermeyer, M.C., Bernert, S., et al. «Prevalence of mental disorders in Europe: results from the European Study of the Epidemiology of Mental Disorders (ESEMeD) project.» Acta Psychiatrica Scandinavica, 2004: 109, 21-27.

5. Anda RF., Craft JB., Felitti VJ. Et al. "Adverse childhood experiences and smoking during adolescence and adulthood". JAMA. 1999 Nov. 282(17):1652-8

6. Anda R., Tietjen G., et al. "Adverse childhood experiences and frequent headaches in adults". Headache. 2010 Oct ; 50(9) :1473-81.

7. Association Internationale des Victimes de l'Inceste: Vécu, état de santé et impact sur la vie quotidienne des victimes [Internet] 2010 [cited 2013 Mar 6];Available from : http://aivi.org/fr/telechargements-adherents/cat_view/96etudes?orderby=dmdate_published

8. Attal, J. «Orientation clinique et recommandations: Etat de stress aigu et Etat de stress post traumatique.» Revue du Praticien, 2009: 59, 969-972.

$\underline{B}$ :

9. Badgley, «Infractions d'ordre sexuel contre des enfants au Canada. Rapport du Comité sur les infractions sexuelles à l'égard des enfants et des jeunes. » Approvisionnements et services, Canada-Ottawa, 1984.

10. Bajos N., Bozon M. et al. «Les violences sexuelles en France : quand la porte se libère » Population et Sociétés 2008;445 :p1-4

11. Banyard V.L., Williams L.M., Siegel J.A. "The long-term mental health consequences of child sexual abuse: an exploratory study of the impact of multiple traumas in a sample of women".

J Trauma Stress. 2001; 14: 697-715

12. Baril Daniel. «Un antibiotique pourrait réduire les troubles anxieux » Hebdomadaire de l'information de l'université de Montréal, Volume 41, Numéro 24, 19 mars 2007. Consultable sur : http://www.iforum.umontreal.ca/Forum/2006-2007/20070319/R 1.html

13. Bartzokis G., Lu P.H., Turner J. et al. « Adjunctive risperidone in the treatment of chronic combat-related posttraumatic stress Disorder" Biol. Psychiatry. 2005; 57: 474-479.

14. Bendall S., Jackson H.J., Hulbert CA. "What slef-generated speech is externally misattributed in psychosis? Testing three cognitive models in a first-episode sample." Schizophrenia Research. 2011; 129(1):36-41.

15. Billete V, Guay S, Marchand A. «Le soutien social et les conséquences psychologiques d'une agression sexuelle:synthèse des écrits.» Santé Mentale au Québec, 2005: 30(2), 101-120. 
16. Birmes P., Brunet A., Benoit M. et al. «Validation of the peritraumatic dissociative experiences questionnaire self-report version in two samples of French-speaking individuals exposed to trauma" European Psychiatry, 2005, 20, 145-151.

17. Bisson J., Ehlers A. et al. 2, «Psychological treatments for chronic post traumatic stress disorder», British Journal of Psychiatry, 2007

18. Black D.A., Heyman R.E., Slep A.M.S. "Risk factor for child sexual abuse" Aggression \& violent Behavior .2001; 6(2-3): 203-229.

19. Blake D.D., Weathers F.W., Naguy N.M. "Clinician rating scale for assessing current and lifetime PTSD: The CAPS-1”. Behavior Therapist. 1990; 13: 187-188

20. Blanchard E.B., Buckley T.C. et al. « Post-traumatic stress disorder and comorbid major depression : Is correlation an illusion? » Anxiety Disorder.1998; 12:21-31

21. Bleiberg KL, Markowitz JC "A pilot study of interpersonal psychotherapy for posttraumatic stress disorder" American Journal of Psychiatry 2005

22. Boscarino JA. "Posttraumatic stress disorder and physical illness: result from clinical and epidemiologic studies" Ann N Y Acad Sci. 2004 Dec

23. Bouhet B., Pérard D., Zorman M. «De l'importance des abus sexuels en France», in Les enfants victimes d'abus sexuels, ss dir. M. Gabel, Paris, Éd. PUF, 285 p 37-53. 1992

24. Bradley R., Greene J. et al« Multidimensional Meta-analysis of psychotherapy for PTSD » en American Journal of Psychiatry, Février 2005

25. Brady K, Pearlstein T, Asnis GM et al. «Efficacy and safety of sertraline treatment of posttraumatic stress disorder: a randomized controlled trial." Jama 2000, 283: 1837-1844.

26. Braun P., Greengerg D., Dasberg H, Lerer B. Core symptoms of post-traumatic stress disorder unimproved by alprazolam treatment. J Clin. Psychiatry. $1990 ; 51: 236-238$.

27. Bremner J., Randall P., Vermetten E. " Magnetic resonance imaging-based measurement of hippocampal volume in posttraumatic stress disorder related to childhood physical and sexual abuse: a preliminary report." Biol Psychiatry. 1997;41:23-32

28. Breslau N, Anthony J. «Gender Differences in the Sensitivity to Posttraumatic Stress Disorder.» Journal of Abnormal Psychology, 2007: 116(3), 607-611.

29. Brewin, C.R.,Andrews,B.etValentine,J.D. «Meta-analysis of risk factors for posttraumatic stress disorder in trauma-exposed adults.» Journal of Consulting and Clinical Psychology, 2000: 68 (5), 748-766.

30. Brezo J., Paris J., Vitaro F. "Predicting suicide attempts in young adults with histories of childhood abuse.” Br J Psychiatry 2008 Aug; 193:134-139.

31. Brom D, Kleber RJ et al. « Brief Psychothérapy for Posttraumatic Stress dissorders », Journal of Consulting and Clinical Psychology, 1989.

32. Brunet A., Weiss D., Metzler T.J., et al. «The peritraumatic distress inventory: a proposed measure of PTSD Criterion A2". American Journal of Psychiatry, 2001, 158:1480-1485.

33. Brunet A., Saint Hilaire A., Jehel L., King S. « Validation of a French Version of Event Scale revised » Canadian Journal of Psychiatry. 2003 ; 48: 56-61 
34. Brunet A., Orr S.P., Tremblay J., al. "Effect of post-retrieval propranolol on psychophysiologic respondind during subsequent script-driven traumatic imagery in post traumatic stress disorder." Journal of Psychiatric Research. 2008 ; 42 : 503-506

$\frac{\mathrm{C}:}{35}$

35. Cadell S, Regehr C., Hemsworth D. "Factor contributing to posttraumatic growth: a proposed structural equation model." Am. J. Orthopsychiatry. 2003; 73(3): 279-287.

36. Cardeña E., Maldonado J. et al «Hypnosis», Journal of Traumatic Stress, 2000,13, 580-584

37. Centre de Recherche sur l'Enfance et l'Adolescence (CREA), étude menée après de 1000 étudiants. Cité par Bouhet et al. 1992

38. CFCV (Collectif Féministe Contre le Viol) « Bilans d'Activités de 1986 à 2000 », Paris, 9 Villa d'Este 75013

39. Chapman DP., Whitfield CL., Felitti VJ. Et al. "Adverse childhood experiences and risk of depressive disorders in adulthood". Journal of Affective Disorders. 2004 Oct; 82(2):217-25.

40. Cheiwess L. «Neurobiologie des états de stress post traumatiques » Actualité en neurologie Septembre 2000

41. Chen LP, Murad MH, Paras ML "Sexual abuse and lifetime diagnosis of psychiatric disorders: systematic review and meta-analysis." Mayo Clin Proc. 2010 Jul;85:618-

42. Chevreul K., Prigent A. et al. "The cost of mental disorders in France ». European Neuropsychpharmacology, Septembre 2012

43. Ciavaldini A. «Les agressions sexuelles-Données Epidémiologique Générales ». Psychopathologie et traitements actuels des auteurs d'agression sexuelle, Conférence de consensus, 2001 Consultable sur : http://psydoc-

fr.broca.inserm.fr/conf\&rm/Conf/confagrsex/RapportsExperts/Ciavaldini.html

44. Cohen H., Kaplan Z. et al. «Repetitive Transcranial Magnetic Stimulation of the right dorsolateral prefrontal cortex in Posttraumatic Stress Disorder: a double-blind, placebocontrolled study" American Journal of Psychiatry en 2004

45. Coid J., Petruckevitch A. et al. "Relation between childhood sexual and physical abuse and risk of revictimisation in women: a cross-sectional survey". Lancet .2001; 358(9280):450-4

46. Connor KM, Sutherland SM, Tupler LA et al. "Fluoxetine in post-traumatic stress disorder. Randomised, double-blind study.” Br J Psychiatry 1999, 175: 17-22.

47. Cougle J-R, Resnick H, Kilpatrick DG. "Does prior exposure to interpersonal violence increase risk of PTSD following subsequent exposure?" Behav Res Ther. 2009 December; 47(12): 1012-1017.

48. Crocq L., Puech D. «L'Amoxapine dans le traitement des états de stress post-traumatiques. » Psychologie Médicales $1992 ; 24$ : 49-53

49. Cuijpers P., Geraedts AS, Van Oppen P. et al. «Interpersonal psychotherapy for depression : a meta-analysis." American Journal of Psychiatry, Juin 2011.

50. Cyrulnik Boris, extrait d'une interview avec Antoine Spire. Le Monde de l'éducation Mai 2001

$\underline{\text { D : }}$

51. Darves-Bornoz J-M, Degiovanni A. et al. « Incestuous rape in adolescence". Fourth International Congress of the International Society for Adolescent Psychiatry (ISAP). 
Athènes, 1995. In « Syndrome traumatique du viol et de l'inceste » de Darves-Bornoz J-M MASSON 1996

52. Darves-Bornoz J.M. « Syndromes traumatiques du viol et de l'inceste » Masson, Paris, 1996

53. Darvez-Bornoz JM., Pierre F. et al. «Screening for psychologically traumatized rape victims.» Eur J Obstet Gyn R B. 1998, 77: 71-5.

54. Davidson J., Kudler H., Smith R. et al. "Treatment of post-traumatic stress disorder with amitriptyline and placebo." Arch. Gen. Psychiat., 1990; 47: 259-66

55. Davidson J., WeislerR.H, Butterfield et al. « Mirtazapine versus placebo in posttraumatic stress disorder: a pilot trial.” Biol. Psychiatry, 2003; 53: 188-191

56. Davidson JR, Rothbaum BO, van der Kolk BA et al. "Multicenter, doubleblind comparison of sertraline and placebo in the treatment of posttraumatic stress disorder."Arch Gen Psychiatry 2001, 58: 485-492.

57. Davidson J., Baldwin D., Stein D.J. et al. "Treatment of posttraumatic stress disorder wtih Venlafaxine extended release: A 6 month randomized controlled trial." Arch. Gen. Psychiatry, Octobre 2006.

58. Davidson P.R, Parker K.C.H, « Eye movement desensitization and reprocessing (EMDR) : a meta-analysis », Journal of Consulting and Clinical Psychology, Avril 2001.

59. De Bellis M., Keshavan M., Clark D. et al. « Developmental traumatology, part II: brain development." Biol Psychiatry. 1999; 45:1271-1284

60. De Berardis D., Serroni N. et al. «Agomelatine for treatment of post traumatic stress disorder : A case report» Annals of Clinical Psychiatry. 2012, 24(3):241-242.

61. Debray Q. "Traumatisme, syndrome psychtraumatique et trouble de personnalité", in Psychotraumatologie: Evaluation, Clinique, traitement » Paris, Dunod, 2006

62. DeFrancis V. «Protecting the child victim of sex crimes comitted by adults », American Human Association, children division. Denver, CO, 1969

63. Denis Daphnée, «Le viol, l'autre arme de destruction massive », Slate.fr, 13/09/2011 Consultable sur: http://www.slate.fr/story/42885/viol-arme-guerre.

64. De Venter M., Demyttenaere K., Bruffaerts R. «The relationship between adverse childhood experiences ans mental health in adulthood. A systematic literature review" Tijdsschr Psyvchiatry 2013; 55(4):259-68.

65. De Villiers Laurent, «Tais-toi et pardonne! », Flammarion, Paris, 2011.

66. Dierks M.R., Jordan J.K., Sheehan A.H. "Prazosin treatment of nightmares related to posttraumatic stress disorder." Ann.Pharmacother. 2007 juin; 41: 1013-1017.

67. Driessen M., Herrmann J., Stahl K. et al. "Magnetic resonance imaging volumes of the hippocampus and amygdala in women with borderline personality disorder and early traumatization." Arch Gen Psychiatry. 2000; 57:1115-1122

68. DSM-IV-TR, « Manuel diagnostique et statistique des troubles mentaux » American Psychiatic Association, version française : Gelfi, Julien-Daniel Masson 
69. DSM-V, les modifications concernant l'ESPT sont consultables sur : http://www.dsm5.org/Documents/PTSD\%20Fact\%20Sheet.pdf

70. Ducrocq F, Viaga G. «Traitement psychopharmacologique de l'état de stress post traumatique ». In De clercq M., Lebigot F., Les traumatismes psychiques. 2001.Masson P287320.

71. Dude SR., Anda RF., Felitti VJ. Et al. "Childhood abuse, household dysfunction, and the risk of attempted suicide throughout the life span: findings from the adverse Childhood Experiences Study" JAMA. 2001 Dec 26, 286(24):3089-96.

72. Dude S.R., Anda R.F., Whitfield C.L. et al."Long-term consequences of childhood abuse by gender of victim." American Journal of Preventing Medicine. 2005; 28(5), 430-438.

Consultable sur: http://citeseerx.ist.psu.edu/viewdoc/download?doi=10.1.1.189.5033\&rep=rep1\&type=pdf

73. Dude SR., Miller JW., Brown DW. "Adverse childhood experiences and the association with ever using alcohol and initiating alcohol use during adolescence." J Adolesc Health, 2006 Apr;38:444.e1-10.

74. Duffy J.D., Malloy P.F. "Efficacy of buspirone in the treatment of post traumatic stress disorder: an open trial" Am; Clin. Psychiatry. 1994; 6: 33-37

$\underline{E}:$

75. Eco-Santé France 2012, d'après les données Drees, Comptes de la Santé. Consultable sur : http://www.irdes.fr/EspaceEnseignement/ChiffresGraphiques/Cadrage/DepensesSante/Agrega tsComptesSante.htm

76. Edgardh K. et Ormstad K. "Prevalence and characteristics of sexual abuse in national sample of Swedish seventeen-year-old boys and girls. " Acta Paediatrica. 2000; 89(3) :310-319

77. Elias S., Louville P., Navarre C. «Traitements médicamenteux de l'état de stress post traumatique: revue de littérature» Actualité Médicale Internationale-Psychiatrie, Décembre 2001.

$\underline{F}:$

78. Felliti VJ., Anda RF., Nordenberg D. et al. "Relationship of childhood abuse and household dysfunction to many of the leading causes of death in adults. The Adverse Childhood Experiences (ACE) Study" American Journal of Preventive Medicine.1998May;14(4):245-58

79. Felitti V.J., Kaiser Permanente MD, Anda RF, , MS Centers for Disease Control and Prevention "The Adverse Childhood Experiences (ACE) Study and prescription drug in a cohort study of adult HMO patients" BCM Public Health 2008

80. Ferenczi Sándor, «Confusion de langue entre les adultes et l'enfant », Petite Bibliothèque Payot, 2004, p. 41-42

81. Fergusson D., Lynskey M., Horwood L. "Childhood sexual abuse and psychiatric disorder in young adulthood, I: prevalence of sexual abuse and factors associated with sexual abuse." $J$ Am Acad Child Adolesc Psychiatry.1996a; 35:1355-1364

82. Fergusson D., Horwood L., Lynskey M. "Childhood sexual abuse and psychiatric disorder in young adulthood, II: psychiatric outcomes of childhood sexual abuse." J Am Acad Child Adolesc Psychiatry (1996b); 35:1365-1374

83. Fergusson DM, McLeod GF, Horwood LJ. "Chilhood abuse and adult developmental outcomes: Findings from a 30-year longitudinal study in New Zealand. Child abuse and neglect. Avril 2013. 
84. Fesler F.A. "Valproate in combat-related post-traumatic stress disorder." J. Clin. Psychiatry. 1991.

85. Finkelhor D. and Baron L. "Hight-Risk children". In D. Finkelhor A sourcebook on child sexual abuse (P60-80). Beverly Hills, CA: Sage Publications. 1986.

86. Finkelhor D., Hotaling G. et al. «Sexual abuse in a national survey of adult men and women: prevalence, characteristics and risk factors." Child Abuse and Neglect.1990; 14(1):19-28.

87. Finkelhor D. «Current information on the scope and nature of child sexual abuse". Future Child. 1994 Summer-Fall; 4(2):31-53

88. Finkelhor D., Berliner L. "Research on the treatment of sexually abused children: a review and recommendations." J Am Acad Child Adolesc Psychiatry. 1995

$34: 1408-1423$

89. Finkelhor D., Moore D. et al. «Sexually abused children in a national survey of parents : methodological issues". Chid Abuse and Neglect. 1997; 21(1):1-9

90. Fiscella K., Kitzman H., Cole R., Sidora K., Olds D. "Does child abuse predict adolescent pregnancy?" Pediatrics. 1998; 101:620-624

91. Fleming J., Mullen P. and Bammer G. "A study of potential risk factors for sexual abuse in childhood”. Child Abuse and Neglect. 1997; 21(1): 49-58.

92. Foa E.B, Riggs D.S, Dancun C.V., Rothbaum B.O. «Reliability and validity of a brief instrument for assessing post-traumatic stress disorder" Journal of Traumatic Stress. 1993; 6: 459-473.

93. Foa E.B, Cashman L., Jaycox L.H, Perry K.. "The validation of a self-report measure of PTSD: the PTSD Diagnostic Scale (PDS)”. Psychological Assessment. 1997; 9: 445-451.

94. Foa Edna, Olasov Rothbaum B. «Traiter le traumatisme du viol » Dunod, Paris, 2012

95. Ford ES, Anda RF, Edwards VJ. "Adverse childhood experiences and smoking status in five states." Prev Med 2011 Sep;53:188-193.

96. Forster P.L., Schoenfeld F.B., Marmar C.R. et al. "Lithium for irritability in posttraumatic stress disorder". Journal of Traumatic Stress. 1995; 8: 143-9

97. Fragoso Margaux, «Tigre, tigre ! », Flammarion, 2012.

98. Frank JB, Kosten TR, Giller EL, Jr., Dan E “A randomized clinical trial of phenelzine and imipramine for posttraumatic stress disorder”Am J Psychiatry 1988, 145: 1289-1291.

99. Frank W., Putnam M.D. "Ten-Yeat Research Update Review: Child Sexual Abuse". J. Am. Child Adolesc. Psychiatry. 2003 ; 42(3):269-278

100. Freud Sigmund. (1919/1984) «Introduction à la psychanalyse des névroses de guerre », In Résultats, Idées, Problèmes. Paris : Presse universitaire de France, P247

101. Freud Sigmund. «Une lettre de Sigmund Freud à Wilhelm Flies». 21 Septembre 1897. Espace Lacan 
102. Freud S. «La première Théorie des névroses » $4^{\mathrm{e}}$ Edition. Quadrige/ Oeuvres complètes de psychanalyse. Presses Universitaires de France, Janvier 2010, Paris

103. Friedman MI. "Toward rational pharmacotherapy for PTSD: an interim report." Am J Psyciatry 1998.

104. Fromm Reed, S. "Total estimated cost of child abuse and neglect in the United States: Statistical evidence". Chicago, IL: Prevent Child Abuse America. 2001. Academic: 122 S. Michigan Avenue • Chicago, IL • 60603 • 630-874-4257

G :

105. Garrabé Jean, «Le Syndrome de Briquet », Perspectives psy, 2009.

106. Gisese A., Thomas M. et al. "The impact of a history of childhood abuse on hospital outcome of affective episodes." Psychiatr. Serv. 1998; 49:77-81

107. Gold S., Lucenko B. et al. "A comparison of psychological/psychiatric symptomatology of women and men sexually abused as children." Child Abuse Neglect. 1999;23:683-692.

108. Goldberg DP. "The detection of psychiatric illness by questionnaire maudsley Monograph”, n²1, Oxford, Oxford university press. 1972.

109. Goldberg DP., Hillier VF. “A scale version of the General Health Questionnaire.”.Psychol Med, 1979, 9: 139-45.

110. Golier JA., Yehuda R., Bierer LM., et al. «The relationship of borderline personality disorder to posttraumatic stress disorder and traumatic events". American Journal of Psychiatry. 2003; 160 (11):2018-24.

111. Gomes-Schwartz B., Horowitz J. et al. (1990), "The aftermath of child sexual abuse 18 months later.” In: Child Sexual Abuse, Gomes-Schwartz B, Horowitz J, Carcharelli A, eds. Newbury Park, CA: Sage, 1990:132-152

112. Grant J., Indermaur D., Thornton J. et al. «Intrafamilial adolescents sex offebder : psychological profile and treatment ». Trends \& issues in crime and criminal justice. 2009. N 375 , Canberra, Australia: Australian Institute of criminology. Consultable sur : http://www.aic.gov.au/documents/D/F/9/\%7BDF967C8E-EE57-4891-993E90E40CF1F7B2\%7Dtandi375.pdf

113. Gupta S, Popli A., Bathurst E. et al. "Efficacy of cyproheptadine for nightmares associated with post-traumatic stress disorder." Compr. Psychiatry 1998; 39: 160-164

$\underline{\mathrm{H}:}$

114. Habetha S., Bleich S., Weidenhammer J. et al. "A prevalence-based approach to societal cots occurring in consequence of child abuse and neglect.", Child and Adolescent Psychiatry and MentalHealth, 2012, 6:35

115. Hammer M.B., Deitsch S.E., Brodrick P.S. et al. Quetiapine treatment in patients with posttraumatic stress disorder: An open trial of adjunctive therapy. J. Clin. Psychopharmacol. $2003 ; 23: 15-20$.

116. HAS ( Haute Autorité de Santé) « Affection psychiatrique de longue durée : Troubles anxieux graves » Guide-affections de longues durées, 2007. Consultable sur: http://www.hassante.fr/portail/upload/docs/application/pdf/guide_medecin_troubles_anxieux.pdf 
117. HAS «Repérage et signalement de l'inceste par les médecins : reconnaitre les maltraitances sexuelles intrafamiliales chez le mineur $\gg$ Recommandations de bonnes pratiques professionnelles, 2011, consultable sur: http://www.hassante.fr/portail/upload/docs/application/pdf/201106/maltraitance sexuelle fiche de synthese. pdf

118. HAS «Commission de la transparence, Avis Mars 2013, Zoloft» Consultable sur : http://www.has-sante.fr/portail/upload/docs/evamed/CT7400_ZOLOFT_PIC_avis1_CT7400.pdf

119. Heijink R., Renaud T. «Etudes des couts par pathologie une comparaison méthodologique entre cinq pays.», Question d'économie de la santé, Juin 2009, n 143

120. Hertzberg MA, Butterfield MI, Feldman ME, et al."A preliminary study of lamotrigine for the treatment of posttraumatic stress disorder." Biol Psychiatry, 1999, 45: 1226-1229

121. Hochard F., Roussel A. «L'hopital face à l'enfant maltraité ». Edition Karthala. Paris. 1997

122. Hoge A, Austin ED, Pollack MH.« Resilience: research evidence and conceptual considerations for posttraumatic stress disorder» Depress Anxiety,2007: 24(2),139-152

123. Holmes W.C and Slap G.B. "Definition, prevalence, correlates, squelae, and management." Journal of the American Medical Association. 1998; 280(21), 1855-1862.

124. Horowitz M.J., Wilner N.R., Alvarez W. "Impact of Event Scale: A measure of subjective distress". Psychometric Medicine. 1979; 41:209-218.

125. Hotte JP., Rafman S. "The specific effects of incest on prepubertal girls from dysfunctional families." Child Abuse Neglect. 1992;16:273-283.

I :

126. IPSOS/AIVI (Association Internationale des Victimes d'inceste). « Etat des lieus de la situation des personnes victimes d'inceste: vécu, état de santé et impact sur la vie quotidienne» 2010. Consultable sur :

http://www.memoiretraumatique.org/assets/files/doc_violences_sex/AIVI_inceste_mai_2010. pdf

127. Irazuzta J.E, McJunkin J.E, Danadian K, et al. "Outcome and cost of child abuse". Child abuse and neglect, 1997, vol. 21, n $\mathrm{n}^{\circ}$, pp. 751-757 Robert C. Byrd Health Sciences Center of West Virginia University/Charleston Division and Women \& Children's Hospital, Charleston Area Medical Center, Charleston, WV, ETATS-UNIS

$\underline{\mathrm{J}:}$

128. Janet Pierre. «L'automatisme psychologique. Essaie de psychologie expérimentale sur les formes inférieures de l'activité humaine ».1889 Document numérique de la collection Les classiques des sciences sociale. Dirigée par Jean Marie Tremblay. Université du Québec à Chicoutimi.

129. Jaspard M. et al. « Nommer et compter les violences envers les femmes une première enquête nationale en France ». Population et société, 2001 Janvier ; n³64

130. Jeammet P Reynaud M., Consoli S. Abrégés:Psychologie médicale. Masson 2é ed. 2004. P230

131. Jehel L., Brunet A., Paterniti S., Guelfi J.D. « Validation de la version française de détresse péritraumatique ». Canadian Journal of Psychiatry.2005, vol 50, n 1, 67-71 
132. Jehel L., Sylvestre M. et Louville P. «Evaluation, orientation et accompagnement : intervention auprès d'une personne souffrant d'un trouble psychotraumatique. » In Psychothérapie des victimes : traitements, évaluations, accompagnement. De Lopez G., Sabouraud-Seguin A., Jehel L. DUNOD $2^{\mathrm{e}}$ edition 2006 (2) : 20-27

133. Jovanovic A., Aleksandric B."Family Hardiness and Social support as predictor of PostTraumatic Stress Disorder" Psychiatry, Psychologie and Law. 2004;11(2): 263-268

$\underline{K}:$

134. Kédia Marianne. «La dissociation : un concept central dans la compréhension du traumatisme ». 2009, L'évolution psychiatrique, 74:487-496.

135. Kessler R.C., Sonnega A. et al. « Posttraumatic stress disorder in National Comorbidity Survey » Archives of General Psychiatry. 1995; 52(1):1048-60

136. Kessler R.C., "Posttraumatic stress disorder: the burden to the individual and to society." Journal of Clinical Psychiatry. 2000; 61 Suppl 5, 4-12; discussion 13-14.

137. Kessler R.C., Bergluand P., et al. « Lifetime prevalence and age-of-onset distributions of DSM-IV disorders in the National Comorbidity Survey Replications. Archives of Genera Psychiatry. 2005; 62:593-603.

138. Kilpatrick D.G., «Rape Aftermath Symptom Test » in Dictionary of behavioral assessment techniques, M. Hersen, A.S. Bellack (éd), Oxford, Pergamon Press 1988.

139. Kinzie J.D., Leung P. "Clonidine in cambodian patients with posttraumatic stress disorder." Journal of Nervous and Mental Disease. 1989; 177: 546-550.

140. Kolb L.C., Burris B.C., Griffith S. "Propranolol and Clonidine in the treatment of posttraumatic stress disorder of war". American Psychiatric press. 1987;p 98-105

141. Kong S., Bernstein K. "Childhood trauma as a predictor of eating psychopathology and its mediating variables in patients with eating disorders". Journal of Clinical nursing. 2009;18:1897-1907. http://www.brown.uk.com/eatingdisorders/kong.pdf

142. Kosten T.R. et al. "Pharmacotherapy for post-traumatic stress disorder using Phenelzine or Imipramine". J Nerv. Dis., 1991; 179: 366-370

143. Kozaric-Kovacic D., Pivac N. Quetiapine treatment in a open label trial in combat-related posttraumatic stress disorder with psychotic features. Int. J. Neuropsychopharmacol. 2007; 10: 253-261.

144. Krupnick JL, Green BL, Stockton P et al. "Group interpersonal psychotherapy for low-income women with posttraumatic stress disorder". Psychotherapy Research 2008

$\underline{L}:$

145. Lebigot F., Daligand L. "Traiter les traumatismes psychique” Paris, DUNOD. 2006

146. Lecrubier Y., Sheehan D., Weiller E., Armorin P. et al. “The MINI International Neuropsychiatric Interview (MINI), a short diagnostic Interview: Reliability and validity according to the CIDI" European Psychiatry. 1997; 12:232-241.

147. Legifrance consultable sur: http://www.legifrance.gouv.fr/ 
148. Lensvelt-Mulders G, van der Hart O, van Ochten JM et al. «Relations among peritraumatic dissociation and posttraumatic stress: A meta-analysis.» Clinical Psychology Review, 2008: 28(7), 1138-51.

149. Levitan R., Parikh S., Lesage A et al. « Major depression in individuals with a history of childhood physical or sexual abuse: relationship to neurovegetative features, mania, and gender." Am J Psychiatry. 1998;155:1746-1752

150. Lindegren ML., Hanson IC. Et al. « Sexual abuse of children: intersection with the HIV epidemic.»Pediatrics. 1998 Oct; 102(4):E46.

151. Linehan M.M. « Traitement cognitivo-comportemental du trouble de personnalité étatlimite ». Guilford press . New-york, 1993.

152. Lipper S. "Carbamazepine in the treatment of posttraumatic stress disorder : implications for the kindling hypothesis." American Psychiatric press, 1990.

153. Lopez G. «Psychotraumatismes majeurs : L'accompagnement social et judiciaire » Confrontations psychiatriques $2012 ; \mathrm{n}^{\circ} 51: 327-344$

$\underline{\mathrm{M}}:$

154. Mamar C.R., Weiss D.S., Metzler T.J. "The Peritraumatic dissociative experiences questionnaire." in Assessing psychological trauma and PTSD: a handbook for practitioners, New York, Guilford Press, 1997: 412-428.

155. Mai F., Merskey H. «Le "traité de l'hystérie" de Paul Briquet», Archives of general psychiatry,1980, $37: 1401-1405$. Consultable sur :

http://www.biusante.parisdescartes.fr/sfhm/hsm/HSMx1982x017xspec2/HSMx1982x017xspe $\underline{\mathrm{c} 2 \mathrm{x} 0071 . \mathrm{pdf}}$

156. Maxfield L., Hyer L. en 2002, dans «The relationship betweem efficacy and methodology in studies investigating EMDR treatment of PTSD” Journal of Clinical Psychology, 2002, Vol 58 .

157. McFarlane AC, Yehuda R. "Clinical treatment of posttraumatic stress disorder: conceptual challenges raised by recent research."Aust N Z Psychiatry. 2000 Dec;"'(6):940-53

158. McKenna K., Ph.D., Bowlus A., Ph.D.’The economic costs and consequences of child abuse in Canada". Report to the Law Commission of Canada, 2003.

$\underline{N}:$

159. Nelson E, Heath A, Madden $P$ et al. "Association between self-reported childhood sexual abuse and adverse psychosocial outcomes: results from a twin study." Arch Gen Psychiatry. 2002; 59:139-146

160. NeumannD., Houskamp B., Pollock V, Biere J. “ The long-term sequelae of childhood sexual abuse in women:a meta-analytic review." Child maltreatment. 1996;1 :6-16.

161. Neumark-Sztainer D, Story M, Hannan $P$ et al. "Disordered eating among adolescents: associations with sexual/physical abuse and other familial/psychosocial factors." International Journal of Eating Disorders 2000; 28: 249-258.

162. Nixon R.D.V., Resick P.A., Nishith P. "An exploration of comorbid depression among female victims of intinatepartner violence with posttraumatic stress disorder" J. Affect. Disord. 2004 $82,315-320$. 
163. Norandotti N., Dima D., Joqia J. «Childhood abuse is associated with strutural impairment in the ventrolateral prefrontal cortex and aggressiveness in patients with borderline personality disorder» Psychiatry Research. Mai 2013.

164. Norman RE., Byambaa M.,et al. « The long-term health consequences of child physical abuse, emotional abuse, and neglect: a systemic review and meta-analysis." PLoS Med. 2012;9(11)

165. Norris FH. "Epidemiology of trauma: frequency and impact of different potentially traumatic events on differents demographic groups." J.Consult.Clin.Psychol. Juin 1992; 60 (3): 409-418

$\underline{\mathbf{0}:}$

166. Obépi-Roche, Enquête nationale sur l'obésité et le surpoids, 2012 consultable sur : http://www.roche.fr/home/recherche/domaines_therapeutiques/cardio_metabolisme/enquete_n ationale obepi 2012.html

167. ODAS (Observatoire national de l'enfance en danger). « Observation de l'enfance en danger pour l'année 1999 ». LA LETTRE. $\mathrm{N}^{\circ}$ spécial Novembre 2000

168. Olff M., Frijling J.L et al. "The role of oxytocin in social bonding, stress regulation and mental health: An update on the moderating effects of context and interindividual differences." Psychoneuroendocrinologie. 12 Juillet 2013.pii: S0306-4530(13)00236-9

169. Oquendo M., Brent D.A., Birmaher B. et al. « Posttraumatic stress disorder comorbid with major depression : factors mediating the association with suicidal belavior » American Journal of Psychiatry. 2005; 162(3):560-566.

170. Ozer EJ, Best SR, Lipsey TL, Weiss DS. "Predictor of posttraumatic stress disorder and symptoms in adults: a meta-analysis". Psychol Bull. Janvier 2003; 129: 52-73

171. Paolucci E, Genuis M., Violato C. «A meta-analysis of the published research on the effects of child sexual abuse." J. Psychol. 2001;135:17-36.

172. Paras M.L, Murad M.H, Chen L.P. "Sexual abuse and lifetime diagnosis of somatic disorders: a systematic review and meta-analysis." JAMA 2009 Aug 5;302:550-561

173. Pariente K. «Théorie et thérapie comportementales et cognitives » Issu de L'aide mémoire de Psychotraumatologie de Kédia Marianne, Sabouraud-séguin A. et al. , Dunod, Paris, 2008, Chap 41, P228.

174. Pariente P., Challita H., Mesba M., Guelfi JD. "The GHQ-28 questionnaire in french : a validation survey in a panel of 158 general psychiatric patients." Eur Psychiat, 1992 ; 7:15-20.

175. Pearlman L. Saaktvivne K. «Trauma and the thearapist » New-York, Norton, 1995

176. Pearlman L.A, Communication personnelle, Octobre 1996

177. Pitman R.K., Sanders K.M., Zusman R.M. et al. "Pilot study of secondary prevention of posttraumatic stress disorder with propranolol.” Biol. Psychiatrie. 2002 ; 51 : 189-192.

178. Polusny M., Follette V. "Long term correlates of child sexual abuse: theory and review of the empirical literature.” Appl. Prev. Psychol. 1995 4:143-166

179. Putnam F., Trickett $P$. "The psychobiological effects of sexual abuse: a longitudinal study." Ann N Y Acad Sci. 1997;821:150-159 
180. Rainey D., Stevens-Simon C., Kaplan D. "Are adolescents who report prior sexual abuse at higher risk for pregnancy?” Child Abuse Neglect. 1995; 19:1283-1288

181. Robertson $M$, Rushhton $P$, Batrim D et al, "Open trial of interpersonal psychotherapy for chronic post traumatic stress disorder”, Australasian psychiatry, octobre 2007

182. Rodriguez M., Perez V., Garcia Y. "Impact of traumatic experiences and violent acts upon response to treatment of a sample of Colombian women with eating disorders." International Journal of Eating Disorders 2005 ; 37 : 299-306.

183. Roenholt S., Beck N.N, Sidsel H. et al. "Post-traumatic stress symptoms and childhood abuse categories in national representative sample for a specific age group: associations to body mass index". European Journal of Psychotraumatologie. 2012 June

184. Rorty M et Yager J. « Histories of childhood trauma and complex post-traumatic sequelae in women with eating disorder. » Psychiatric Clinics of North America. 1996; 19:773-791.

$\underline{S}$ :

185. Sack M., Lempa W., Lamprecht F. «Study quality and effect sizes-a meta-analysis of EMDR treatment for post traumatic stress disorder" Psychotherapy, psychosomatic Medizinische Psychologie,2001, Vol 51

186. Sarason I., Sarason B., Shearin E., Pierce G. « A brief measure of social support : practical and theorical Implications". Journal of Social and Personnal Relationships. 1983. 4 : 497-510

187. Schoenfeld F.B., Marmar C.R., Neylan T.C. "Current concepts in pharmacotherapy for posttraumatic stress disorder." Psychiatric Services. 2004; 55: 519-31

188. Shalev AY, Freedman S, Peri T, Brandes D, Sahar T, Orr SP, Pitman RK. "Prospective study of posttraumatic stress disorder and depression following trauma" Am J Psychiatry. 1998 May; 155(5):630-7

189. Sheehan D.V, Lecrubier Y., Sheehan K.H. et al. "The MINI International Neuropsychiatric Interview (MINI): The development and validation of a structured diagnostic Psychiatric Interview for DSM-IV and ICD 10”. Journal of Clinical Psychiatry, 1998, 59(Suppl.20), 22$33,34-57$.

190. Shin LM, Whalen PJ, Pitman RK et al. «An MRI study of anterior cingulate function in posttraumatic stress disorder» Biological Psychiatry, 2001: 50,932-42.

191. Sideli L., Mule A. et al. « Do child abuse and maltreatment increase risk of schizophrenia?" Psychiatry Investigation. 2012 Juin; 9(2):87-99

192. Sigward J-M. "Conduites addictives et événements traumatiques" in Jehel L., Lopez G. et al. Psychotraumatologie : évaluation, clinique, traitement. Dunod, Paris, 2006.

193. Simon Victor «l'Hypnose dans le traitement du psychautraumatisme » issu de Psychothérapie des victimes : traitements évaluations, accompagnement de Lopez, Sabouraud-seguin, Jehel. DUNOD, Paris, 2006. p145-146

194. SNATEM (Service National d'Accueil Téléphonique pour l'Enfance Maltraitée) « Rapports d'activité », 1999 et 2001. 63 boulevard Bessières, PARIS

195. Spitzer R., Williams J.B., Gibbon M., Fist M.B. "Structural Clinical Interview for DSM-IV" Washington DC, American Psychiatric Press, 1990. 
196. Steil R., Straube E.R. « Post-traumatic stress disorder in children and adolescents ». Zeitschrift fur Klinische Psychologie und Psychotherapie. 2002;31:1-13

197. Stein MB., Barrett-Connor E. "Sexual assault and physical health: finding from a populationbased study of older adults". Psychosomatic Medicine. 2000 Nov-Dec;62(6):838-43

198. Stein MB, Jang KL, Taylor S, Vernon PA, Livesley WJ. «Genetic and environmental influences on trauma exposure and posttraumatic stress disorder symptoms: a twin study.» Am J Psychiatry, 2002: 159(10), 1675-81.

199. Stein M.B., Kline N.A., Matloff J.L. Adjunctive olanzapine for SSSRI-resistant combat related PTSD: A double blind placebo controlled study? Am. J. Psychiatry. 2002 ; 159: 17771779 .

200. Stephenson KR., Hughan CP., Meston CM. "Childhood sexual abuse moderates the association between sexual functioning and sexual distress in women." Child abuse and neglect. 2012 36(2) : 180-9

201. Stevens-Simon C., Reichert S. "Sexual abuse, adolescent pregnancy, and child abuse." Arch Pediatr Adolesc Med. 1994;148:23-27

202. Stier D.M., Leventhal J.M., Berg A.T. et al. "Are children born to young mother at increased risk of maltreatment?” Pediatrics. 1993 ; 91(3) : 642-648.

203. Sullivan PF, Bulik CM, Carter FA. "The significance of a history of childhood sexual abuse in bulimia nervosa." British Journal of Psychiatry. 1995 Nov;167:679-682.

$\underline{T}:$

204. Taylor P., Moore P., Pezzullo L., et al. "The Cost of Child Abuse in Australia", Australian Childhood Foundation and Child 2008, Abuse Prevention, Research Australia: Melbourne

205. «Thérapie de l'Etat de Stress Post-Traumatique par Exposition Prolongée. Soigner les victimes de viol. » Foa Edna pour l'APACS, 15 Octobre 2012 Université Paris V. PARIS

206. Tison P. «Les thérapies comportementales et cognitives dans les conduites d'alcoolisation ». Alcoologie et addictologie. 2002, vol. 24, $\mathrm{n}^{\circ} 4$, pp. 345-358

207. Tourigny M. "Incidence, facteurs de risqué et programmes de prévention des abus sexuels envers les enfants". Recueil des études commandées par le Groupe de travail pour les jeunes. Quebec : Ministère de la santé et des Services sociaux. 1991

208. Tourigny M., Hébert M., Joly J. et al. « Prevalence and co-occurrence of violence against children in the Quebec population." Australian and New Zealand Journal of Public Health, 2008; 32(4), 331-335.

209. Trickett P., Noll J., et al. "Variants of intrafamilial sexual abuse experiences: implications for short- and long-term development." Dev Psychopathol. 2001;13:1001-1019

210. Tucker P, Zaninelli R, Yehuda R et al. « Paroxetine in the treatment of chronic posttraumatic stress disorder: results of a placebo-controlled, flexibledosage trial." J Clin Psychiatry 2001, 62: $860-8$

211. Tucker P., Potter-Kimball R., Wyatt D.B. et al. "Can physiologic assessment and side effects tease out differences in PTSD trials? Double-Blind comparison of citalopram, sertraline and placebo", Psycho. Pharmacol.Bull, 2003 
212. Tyrka A., Wyche M., Kelly M.et al. "Childhood maltreatment and adult personality disorder symptoms: influence of maltreatment type" Psychiatry Res. 2009; 28: 165(3): 281-287

Consultable sur: http://www.ncbi.nlm.nih.gov/pmc/articles/PMC2671800/\#R7

$\underline{V}:$

213. Vairel Gilles, «Viols de guerre, stratégie de guerre!», Le Monde.fr, 07/01/2009. Consultable sur: http://www.lemonde.fr/idees/chronique/2009/01/08/viols-de-guerre-strategie-deguerre 1139078 3232.html

214. Vaiva G., Ducrocq F., Jezequel K. et al. «Immediat treatment with propranolol decreases post-traumatic stress disorder two months after trauma." Biol. Psychiatry. 2003; 54 : 947-949.

215. Vaiva G, Jehel L, Cottencin O, Ducrocq F. et al. «Prevalence of trauma-related disorders in the French WHO study: Santé mentale en population générale (SMPG).» Encephale. 2008: $34(6), 577-83$.

216. Vaiva G. «Santé mental en population générale », 2003 cité dans « Psychotrauma en chiffre : des enjeux multiples » François Ducrocq ». Stress et trauma. 2009 ; 9(4) :199-200

217. Van der Kolk BA, Dreyfuss D, Michaels M et al. "Fluoxetine in posttraumatic stress disorder”. J Clin Psychiatry 1994, 55: 517-522.

218. Van Etten M.L., Taylor S. «Comparative efficacy of treatments for post traumatic disorder: A meta-analysis » Clinical Psychology and Psychothérapy, 1998 Vol.5.

219. Van Winkel R., Van Nierop M. et al. "Childhood trauma as a cause of psychosis: linking genes, psychology and biology" Canadian Journal of Psychiatry. Janvier 2013; 58(1):44-51.

$\underline{\text { W: }}$

220. Walker EA., Unutzer J. et al. "Cost of Health Care Use Women HMO Members With a history of Childhood abuse and neglect", Arch Gen Psychiatry, July 1999, Vol 56

221. Walsh K., Danielson C.K et al. «National Prevalence of Posttraumatic Stress Disorder Among Sexually Revictimized Adolescent, College and Adult Household-Residing Women.» Archives of General Psychiatry. 2012 ; 69(9) :935-42.

222. Wang C-T, Holton J, Ph.D. "Total estimated cost of child abuse and neglect in the United States: Economic impact study".. This report was funded by the Pew Charitable Trusts. Prevent Child Abuse America Chicago, Illinois, September 2007

223. Weathers F.W., Litz B.T., Herman B.S. «The PTSD Checklist: reliability and diagnostic utility», paper presented at the International Society for Traumatic Stress Studies, conference San Antonio. 1993

224. Weiss D.S., MarmarC.R. "The impact of event scale revised", in Wilson J.P. et Keane T.M. Assessing Psychological Trauma and PTSD: A handbook for practionners, New-York, Guilford Press, 1997: 399-411

225. Whitfield CL., Dube SR., Felitti VJ. "Adverse childhood experiences and hallucinations." Child Abuse Neglect. 2005 Jul;29:797-810

226. Widom C., Ames M. "Criminal consequences of childhood sexual victimization." Child Abuse Neglect. $1994 ; 18: 303-318$

227. Wolfe, V. V. "Child sexual abuse". In E. J. Mash \& R. A. Barkley (Eds.), Assessment of childhood disorders (4th ed.) (p. 685-748). New York, NY: Guilford Press. 2007 
228. Wonderlich SA, Brewerton TD, Jocic Z et al. "Relationship of childhood sexual abuse and eating disorders. "Journal of the American Academy of Child and Adolescent. 1997 Psychiatry 36: $1107-15$.

229. Wonderlich SA., Crosby RD et al. « Sexual Trauma and personality: developemental vulnérability ans additive effects." J Pers Disord. 2001;15(6):496-504.

230. World Federation of Societies of Biological Psychiatry (WFSBD) "Guidelines for pharmacological treatment of anxiety, obsessive-compulsive Anxiety, obsessive-Compulsive and Posttraumatic stress Disorders." Bandelow B, Zohar J, Hollander E et al, World J Biol Psychiatry 2002; 3: P171-199

231. Wyatt G.E., Myers H.F., Williams J.K., et al. "Does a history of trauma contribute to HIV risk for Women of Color? Implications for Prevention and Policy." American Journal of Public Health. 2002 April; 92(4): 660-665. http://www.ncbi.nlm.nih.gov/pmc/articles/PMC1447133/

$\underline{Y}:$

232. Yao S-N., Cottraux J., Note I. et al. « Evaluation des états de stress post-traumatique : validation d'une échelle, la PCLS » Encéphale. 2003; 29:232-238.

233. Yehuda R. et al. "Hypothalamic-Pituitary adrenal dysfunction in PTSD." Biol Psychiatry, 1991, 30

234. Yehuda R. et al. "Enhanced suppression of cortisol following a low dose of dexamethasone with post traumatic stress disorder." Am J Psychiatry 1993

$\underline{\mathbf{Z}:} \quad$ Zanarini M.C., Ruser T.F. et al. « Risck factors associated with dissociated experiences of borderline patients ». Journal of Nervous and mental Disease. 2000; 188:26-30.

236. Zeig Jeffrey "Experiencing Erickson: An Introduction to the Man and His Work", Brunner/ Mazel, New-York, 1985.

237. Zierler S, Feingold L, Laufer D, Velentgas $P$, et al. "Adult survivors of childhood sexual abuse and subsequent risk of HIV infection." Am J Public Health. 1991;81:572-575

238. Zigmond AS., Snaith RP. "The Hospital Anxiety and Depression Scale.” Acta Psychiatr Scand. 1983; 67: 361-370.

239. Zlotnick C., Mattia J., Zimmerman M. "Clinical features of survivors of sexual abuse with major depression.” Child Abuse Neglect. 2001;25:357-367

240. Zohar J, Amital D, Miodownik C, Kotler M et al. « Double-blind placebo-controlled pilot study of sertraline in military veterans with posttraumatic stress disorder." J Clin Psychopharmacol 2002, 22: 190-195. 199

241. Zoellner LA, Foa EB, Brigidi BD. «Interpersonal friction and PTSD in female victims of sexual and nonsexueal assault". J Trauma stress. Oct 1999; 12(4):689-700. 


\section{Sources:}

Confrontation Psychiatrique ${ }^{\circ}$ 51, Psychotraumatismes majeurs. Sanofi

Darves-Bornoz J.M « Syndrome traumatiques du viol et de l'inceste » Publié par le congrès de psychiatrie et de Neurologie de langue française. Masson, Paris, 1996.

Foa E., Keane T., Friedman M. «Effective treatments for PTSD practice Guidelines from the International society for Traumatic stress studies second edition" The Guilford Press, New-York 2009

Guay Stéphane et Marchand André « Les troubles liés aux évènements traumatiques : dépistage, évaluation et traitement » Les presses de l'Université de Montréal. 2006

Hebert M., Cyr M. Tourigny M. «L'agression sexuelle envers les enfants, Tome 1 » Les presses de l’Université du Quebec. Broché, 2011.

Jehel Louis, Lopez Gérard « Psychotraumatologie : évaluation, clinique, traitement » Dunod, Paris, 2006

Kedia Mariane, Sabouraud-Seguin Aurore « L'aide-mémoire : psychotraumatologie » Dunod, Paris, 2008.

Lopez Gérard, Sabouraud-Séguin Aurore, Jehel Louis. «Psychothérapie des victimes : traitements, évaluations, accompagnement $» 2^{\mathrm{e}}$ édition, Dunod, Paris, 2006.

Vaiva G., Lebigot F., Ducrocq F., Goudemand M. «Psychotramatisme : prise en charge et traitements » Masson, Paris, 2005. 
ANNEXES 


\section{Document annexe 1}

Hétéro-questionnaire : données sociodémographiques, santé globale, recensement des dépenses de santé

\section{Données socio démographiques}

.Quel est votre lieu de naissance ? (pays, département, ville)

Quelle est votre date de naissance ? (Jour/Mois/Année)

Sexe: Homme [_] Femme [_]

. Département et Ville de résidence actuels :

Quel est votre statut matrimonial ?

$\begin{array}{ll}\text { [_] } & \text { Célibataire } \\ \text { [_] } & \text { Marié(e) } \\ \text { [_] } & \text { Pacsé(e), en concubinage } \\ \text { [_] } & \text { Veuf (Veuve) } \\ \text { [_] } & \text { Divorcé(e) }\end{array}$

Combien d'enfants avez-vous?

Cochez la réponse qui correspond à votre situation actuelle

.Votre logement vous en êtes :

[_] Propriétaire

[_] Locataire

[_] Je vis au domicile de mes parents ou d'un membre de ma famille

[_] Autre, précisez

.Quel est votre statut professionnel actuel ?

[_] Actif

Dans ce cas, quelle profession exercez-vous?

[ ] Retraité

Dans ce cas,

A quel âge avez-vous pris votre retraite?

Quelle profession exerciez-vous?

[_] Chômeur, Inactif

Dans ce cas,

Depuis combien de temps êtes-vous au chômage?

Quel est votre niveau d'études?

[_] Homme ou Femme au foyer

Dans ce cas,

Quel est votre niveau d'études?

[_] Etudiant

Dans ce cas, quel est votre niveau d'études ?

. Pouvez vous nous indiquer vos revenus nets par mois 


\section{Prestations sociales:}

.Etes-vous pris en charge à $100 \%$ ?

OUI [_] NON [_]

Si oui, pour quelle(s) pathologie(s) ?

.Actuellement, avez-vous la $\mathrm{CMU}$ (couverture maladie universelle) ?

OUI [_] NON [_]

.En dehors de la CMU complémentaire, bénéficiez vous d'une couverture complémentaire maladie (Mutuelle, Assurance, Caisse de prévoyance) ?

OUI [_] NON [_]

.Quelles sont la ou les prestations que vous percevez actuellement :

[_] Allocation chômage

[_] Allocation familiale

[_] Allocation pour jeune enfant (APE ou PAJE)

[_] Le RSA

[_] Une prestation relative à une invalidité ou à un handicap (AAH, Pension d'invalidité, minimum invalidité, AES)

[_] Rentes accident du travail et maladie professionnelle

[_] Minimum vieillesse

[_] Prestation dépendance (APA, PSD)

[_] Aides financières au logement

[_] Autres (dont allocation veuvage, congés maladie longue durée)

Précisez :

.Quel est le montant total mensuel des prestations dont vous bénéficiez ?

\section{SANTE}

.Poids en $\mathrm{Kg}$ :

.Taille en $\mathrm{Cm}$ :

IMC :

.Actuellement, avez vous une ou des pathologies (ex : HTA, Diabète, insuffisance cardiaque, dépression...)?

Oui [_] Non [_]

De quoi s'agit-il ?

\section{. Consommations de toxiques}

Parmi les substances ci-dessous, avez-vous déjà utilisé l'une d'entre elles ?

( $Y$ compris quand vous étiez adolescent)

Tabac (ex : cigarette, cigare, pipe) : Oui [_] Non [_]

Si oui :

A quel âge avez vous commencé à fumer :

Combien de cigarettes par jour actuellement : 
Boissons alcoolisées (y compris bière, vin, liqueur) : Oui [_] Non [_]

Si oui :

A quel âge avez vous commencé :

Actuellement votre consommation est-elle juste occasionnelle voire nulle ? Oui [_] Non [_] Si non, Combien de verres par jour :

\section{Cannabis : Oui [ ] Non [ ]}

Si oui, A quel âge avez vous commencé :

Actuellement votre consommation est-elle : Occasionnelle $(<5 \mathrm{~J} / \mathrm{Mois})\left[\_\right] \quad$ Régulière [_] Nulle [_]

Combien de joints par jour ou par semaine:

Cocaïne ou Crack

Stimulants (amphétamines, anorexigènes)

Solvants (nitrates, colle, peinture, solvant, gasoil)

Hallucinogènes (LSD, acide, PDP...)

Héroïne, Morphine, Méthadone

Sédatifs ou hypnotiques

(En dehors de prescriptions médicales)

$\begin{array}{lc}\text { Oui [_] } & \text { Non [_] } \\ \text { Oui [_] } & \text { Non [_] } \\ \text { Oui [_] } & \text { Non [_] } \\ \text { Oui [_] } & \text { Non [_] } \\ \text { Oui [_] } & \text { Non [_] } \\ \text { Oui [_] } & \text { Non [_] }\end{array}$

Autres, spécifiez :

\section{Consommation médicale :}

\section{Consommation de médicaments}

. Au cours de cette semaine ( 7 jours précédents), avez-vous consommé des médicaments ?

Ne pas oublier les pilules, autres contraceptifs, somnifères, antalgiques, injections, vaccins, patch, crèmes, pommades etc. ...

Si oui :

$$
\text { OUI [_] NON [_] }
$$

Donnez le nom précis de ces médicaments, leur dosage, le problème de santé pour lequel vous avez pris ce médicament, s'il a été prescrit ou s'il s'agit d'automédication, la quantité consommée

\begin{tabular}{|l|l|l|l|l|}
\hline $\begin{array}{l}\text { Nom du produit, } \\
\text { dosage, forme } \\
\text { galénique } \\
\text { (goutte } \\
\text { comprimé, } \\
\text { gélule...) }\end{array}$ & $\begin{array}{l}\text { Problème de } \\
\text { santé pour } \\
\text { lequel vous avez } \\
\text { pris ce } \\
\text { médicament }\end{array}$ & $\begin{array}{l}\text { Nombre de } \\
\text { prises dans la } \\
\text { semaine }\end{array}$ & $\begin{array}{l}\text { Quantité } \\
\text { consommée à } \\
\text { chaque prise } \\
\text { (si elle est } \\
\text { différente d'une } \\
\text { prise à l'autre, } \\
\text { précisez) }\end{array}$ & $\begin{array}{l}\text { Le médicament } \\
\text { avait-il été } \\
\text { obtenu avec une } \\
\text { ordonnance ? }\end{array}$ \\
\hline $\begin{array}{l}\text { Exemple: } \\
\text { Paracétamol, } \\
\text { Comprimé, } \\
500 m g\end{array}$ & Maux de tête & 3 & $2 c p$ & $\begin{array}{l}\text { Oui [_] } \\
\text { Non [x] }\end{array}$ \\
\hline & & & & Oui [_] \\
Non [_]
\end{tabular}


Combien de fois avez-vous consulté un médecin généraliste ces 12 derniers mois ?

Tarif :

Vous a-t-il prescrit des examens complémentaires? Oui [_] non [_]

.Si oui, citez les :

Combien de fois avez-vous eu recours à une consultation aux urgences ces 12 derniers mois ?

.Avez vous eu des examens complémentaires? Oui[_] non [_]

.Si oui, citez les :

Heure de consultation

Combien de fois avez-vous été hospitalisé au cours des 12 derniers mois ?

.Dans quel service ? (spécialité du service et nom de l'établissement)

Durée d'hospitalisation

Examens complémentaires réalisés

Avez-vous bénéficié d'un acte chirurgical au cours des 12 derniers mois ? Oui [_] non [_]

Si oui, citez le/les :

Combien de fois avez-vous consulté un médecin spécialiste ces 12 derniers mois ?

.Spécialiste, lieu de consultation :

Nombre de consultations Tarif

Examens complémentaires :

Spécialiste:

Nombre de consultations Tarif

Examens complémentaires :

Combien de fois avez-vous consulté en cabinet ou en consultation hospitalière un autre professionnel de santé au cours des 12 derniers mois?

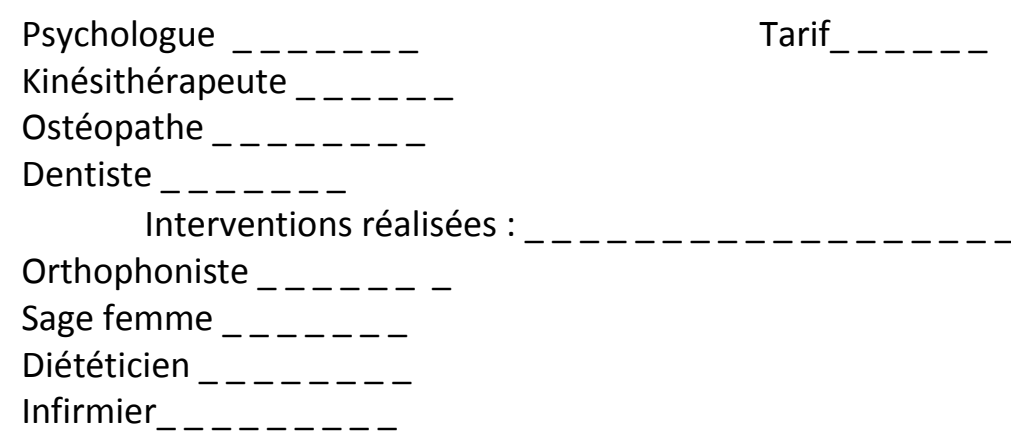

Autres: 
Au cours des 12 derniers mois avez-vous bénéficié d'une prise en charge paramédicale à domicile? OUI [_]

NON [_]

Si oui :

[_] Kinésithérapeute

Nombre de prises en charge :

par semaine,

Pendant _._-__- mois,

Chacune d'environ _._-_._ heure(s).

[_] Infirmier

Nombre de prises en charge :

- _-_-_-_ par semaine,

Pendant _._.___ mois,

Chacune d'environ _._._. heure(s).

[_] Aide soignant

Nombre de prises en charge :

- - - _- _- par semaine,

Pendant

Chacune d'environ _._._. heure(s).

[_] Autre :

Chacune d'environ _- - - - heure(s).

Nombre de prises en charge :

- - - _-_- par semaine,

Pendant _._._._ mois,

Chacune d'environ _._._._ heure(s).

Combien de fois avez-vous fait appel à SOS médecin au cours des 12 derniers mois ?

Avez-vous bénéficié d'une cure thermale au cours des 12 derniers mois ?

Oui [_] non [_]

\section{Consommation de biens médicaux au cours des 12 derniers mois}

Au cours des 12 derniers mois avez-vous bénéficié des services d'un opticien pour des lunettes ? OUI [_] NON [_]

Nombre de paires de lunettes sur l'année Tarifs :

Troubles: Myopie [_]

Hypermétropie [ ]

Presbytie [ ]

Astigmatisme [

Autres

Votre correction $\mathrm{OD}$ :

OG :

Au cours des 12 derniers mois avez-vous nécessité une prothèse ou orthèse (Prothèse auditive, orthèse de repos, attelle de genou, chevillière...). Si oui pouvez vous indiquez leurs tarifs ? 


\section{Transport de malade au cours des 12 derniers mois}

Au cours des 12 derniers mois avez-vous du faire appel

[_] Pompier

Nombre d'interventions :

[_]SAMU

Nombre d'interventions :

Au cours des 12 derniers mois avez-vous bénéficié d'un transport par véhicule sanitaire ? OUI [_] NON [_]

Si oui,

[_] Véhicule sanitaire léger (VSL)

Donner les points de départ et d'arrivée ou donner le nombre approximatif de kilomètres des transports (un aller-retour $=2$ transports)

[] Ambulance:

Donner les points de départ et d'arrivée ou donner le nombre approximatif de kilomètres des transports (un aller-retour $=2$ transports)

[_] Autre transport pris en charge (par exemple trajet en train) et donner le tarif :

\section{Services de médecine préventive au cours des 12 derniers mois}

$>$ Avez-vous consulté un service de médecine préventive ces 12 derniers mois ? (médecine du travail, PMI, médecine scolaire, planning familial...) Oui [_] non [_]

Le(s)quel(s) et nombre de consultations :
. Vous ont-ils prescrit des examens complémentaires ?
Oui [_] non [_]

Si oui, citez les :

\section{Indemnités au cours de 12 derniers mois}

Au cours des 12 derniers mois, avez-vous eu au moins un arrêt de travail de plus de 3 jours ?

OUI [_] NON [_]

Si oui,

Nombre d'arrêts de travail

Durée d'arrêt : Motif

\section{Autres}

Au cours de 12 derniers mois avez-vous bénéficié d'autres examens complémentaires que ceux cités précédemment ? Oui [_] non [_]

Si oui, énumérez-les : 
Y a-t-il eu au cours des 12 derniers mois d'autres actes/dépenses en lien avec votre santé que nous aurions omis de vous demander?

\section{Evènements de vie :}

. Avez-vous avant l'âge de 15 ans été victime de viol ?

«Tout acte de pénétration sexuelle, de quelque nature qu'il soit, commis sur la personne d'autrui par violence, contrainte, menace ou surprise » selon l'article 222-23 du Code pénal

OUI [_] NON (autres violences sexuelles) [_]

\section{Si oui}

.Quel âge aviez- vous lors de cette ou ces agression(s)?

.Quel âge avait votre agresseur (approximativement) ?

. Votre agresseur était-il une connaissance?

Oui [_] Non [_]

.L'agresseur était il un membre de votre famille?

Oui [_] Non [_]

.Quel lien aviez-vous avec votre agresseur?

. A quel âge avez-vous bénéficiez de votre première prise en charge psychiatrique ou psychologique?

.Avez-vous au cours de votre vie été victime d'autres évènements traumatisants ?

Oui [_] Non [_]

Si oui

Nommez-le/les :

Fait-le :

Sujet $\mathrm{N}^{\circ}$ : 


\section{Document annexe 2 :}

\section{Beck Depression Inventory (BDI), Echelle de Dépression de Beck}

Dans chaque série (A, B, C....) entourez la proposition qui correspond le plus à votre état actuel

A $\quad 0 \quad$ Je ne me sens pas triste.

1 Je me sens triste.

2 Je ressens une tristesse continuelle dont je ne parviens pas à me défaire.

3 Je suis si triste et si malheureux que je ne peux presque pas le supporter.

B $\quad 0 \quad$ Je n'envisage pas l'avenir avec une appréhension particulière.

J'envisage l'avenir avec appréhension.

2 Je ne vois rien qui puisse me réjouir.

3 J'ai le sentiment qu'il n'y a rien à espérer de l'avenir et que la situation ne s'améliorera pas.

C $\quad 0 \quad$ Je ne me considère pas comme un raté.

1 J'ai le sentiment d'avoir connu plus d'échecs que la moyenne des gens.

2 Quand je considère ma vie passée, je n'aperçois que des échecs.

3 J'ai le sentiment d'être un parfait raté.

D. 0 J'apprécie les choses exactement comme autrefois.

Je n'apprécie plus les choses comme jadis.

Je ne retire plus aucune satisfaction de quoi que ce soit.

Tout me déçoit ou m'ennuie.

E $\quad 0 \quad$ Je n'éprouve pas de sentiment de culpabilité.

1 J'éprouve parfois un sentiment de culpabilité.

2 J'éprouve souvent un sentiment de culpabilité.

3 J'éprouve constamment un sentiment de culpabilité.

F $\quad 0 \quad$ Je n'ai pas le sentiment d'être puni.

1 J'ai le sentiment que je serai peut-être puni.

2 Je m'attends à être puni.

3 J'ai le sentiment d'appartenir à la catégorie des punis.

G $\quad 0 \quad$ Je ne suis pas déçu de moi-même.

1 Je suis déçu de moi-même.

2 Je me trouve épouvantable.

3 Je me hais.

H $\quad 0 \quad$ Je n'ai pas le sentiment d'être pire que les autres.

1 Je me reproche mes fautes et mes faiblesses.

2 Je me fais constamment des reproches à cause de mes manquements.

3 Je me sens responsable de tout ce qui va mal dans ma vie.

I $\quad 0 \quad$ Je n'ai jamais songé à me faire du mal.

$1 \quad$ Il m'arrive de penser au suicide, mais je ne le ferai pas.

2 Je préférerais me supprimer.

3 Je me supprimerais si je le pouvais.

J $\quad 0 \quad$ Je ne pleure pas davantage qu'autrefois.

Je pleure davantage qu'autrefois.

2 En ce moment. je pleure sans arrêt.
3 Autrefois, j'arrivais à pleurer; aujourd'hui, j'aimerais, mais je ne le peux pas.

K $\quad 0 \quad$ Je ne suis pas plus irritable que d'habitude.

1 En ce moment, je m'énerve ou m'irrite plus vite que d'habitude.

2 Je me sens constamment irrité.

3 Ce qui m'irritait autrefois me laisse totalement indifférent aujourd'hui. 
L $\quad 0 \quad$ Je n'ai pas perdu l'intérêt à l'égard des autres.

Je m'intéresse moins aux autres qu'autrefois.

2 J'ai perdu presque tout l'intérêt que je portais aux autres.

3 J'ai perdu tout l'intérêt que je portais aux autres.

M $\quad 0 \quad$ Je suis toujours aussi décidé qu'autrefois.

1 Je repousse les décisions plus souvent qu'autrefois.

2 J'éprouve beaucoup plus de mal qu'autrefois à prendre des décisions.

3 Je suis incapable de prendre la moindre décision.

$\mathrm{N} \quad 0 \quad$ Je n'ai pas l'impression de paraître moins bien qu'avant.

1 Je crains de paraitre vieux ou moins attractif qu'autrefois.

2 J'ai le sentiment que les changements intervenus dans mon aspect me rendent moins attrayant.

3 Je me trouve repoussant.

$\mathrm{O} \quad 0 \quad$ Je travaille avec autant de facilité qu'autrefois.

1 Je dois me secouer avant d'accomplir une tâche.

2 Je dois me forcer pour le moindre travail.

3 Je suis incapable de travailler.

P $\quad 0 \quad$ Je dors aussi bien qu'autrefois.

1 Je ne dors plus aussi bien qu'autrefois.

2 Je me réveille une heure ou deux plus tôt qu'autrefois, et Il m'est difficile de rendormir.

3 Je me réveille plusieurs heures plus tôt qu'autrefois et ne peux plus me rendormir.

Q $\quad 0 \quad$ Je ne me fatigue pas davantage qu'autrefois.

1 Je me fatigue plus vite qu'autrefois.

2 Presque tout me fatigue.

3 Je suis trop fatigué pour faire quoi que ce soit.

$\mathrm{R} \quad 0 \quad$ Je n'ai pas moins d'appétit qu'autrefois.

1 J'ai moins d'appétit qu'autrefois.

2 Mon appétit est moindre qu'autrefois.

3 Je n'ai plus d'appétit du tout.

$\mathrm{S} \quad 0 \quad$ Je n'ai pratiquement pas maigri ces derniers temps.

1 J'ai perdu plus de deux kilos.

2 J'ai perdu plus de cinq kilos.

3 J'ai perdu plus de huit kilos.

T $\quad 0 \quad$ Je ne me soucie pas davantage qu'autrefois de ma santé.

1 Je m'inquiète de mes problèmes de santé, tels que les douleurs, les ennuis gastriques ou la constipation.

2 Je m'inquiète beaucoup de mes problèmes de santé, et il m'est difficile de penser à autre chose.

3 Je m'inquiète tellement de mes problèmes de santé qu'il m'est impossible de penser à autre chose.

U $\quad 0 \quad$ Je n'ai pas constaté de changement dans ma vie sexuelle, ces derniers temps.

1 Ma vie sexuelle m'intéresse moins qu'autrefois.

2 Ma vie sexuelle m'intéresse beaucoup moins qu'autrefois.

3 J'ai perdu tout intérêt aux questions sexuelles.

TOTAL : 
Document annexe 3 : Impact of Event Scale-Revised, IES-R version française

Instructions : Voici une liste de difficultés que les gens éprouvent parfois à la suite d'un événement stressant.

Veuillez lire chaque item et indiquer à quel point vous avez été boulversé(e) par chacune de ces difficultés au cours des 7 derniers jours en ce qui concerne

Dans quelle mesure avez-vous été affecté(e) ou bouleversé(e) par ces difficultés.

\begin{tabular}{|c|c|c|c|c|c|}
\hline & $\begin{array}{l}\text { Pas du } \\
\text { tout }\end{array}$ & $\begin{array}{l}\text { Un } \\
\text { peu }\end{array}$ & Moyennement & Passablement & Extrêmement \\
\hline $\begin{array}{l}\text { 1. Tout rappel de l'événement ravivait mes } \\
\text { sentiments face à l'événement }\end{array}$ & 0 & 1 & 2 & 3 & 4 \\
\hline 2. Je me réveillais la nuit & 0 & 1 & 2 & 3 & 4 \\
\hline 3. Différentes choses m'y faisait penser & 0 & 1 & 2 & 3 & 4 \\
\hline 4. Je me sentais irritable et en colère & 0 & 1 & 2 & 3 & 4 \\
\hline $\begin{array}{l}\text { 5. Quand j'y repensais ou qu'on me le } \\
\text { rappelait, j'évitais de me laisser } \\
\text { bouleverser }\end{array}$ & 0 & 1 & 2 & 3 & 4 \\
\hline 6. Sans le vouloir, j'y repensais & 0 & 1 & 2 & 3 & 4 \\
\hline $\begin{array}{l}\text { 7. J'ai eu l'impression que l'événement } \\
\text { n'était jamais arrivé ou n'était pas réel }\end{array}$ & 0 & 1 & 2 & 3 & 4 \\
\hline $\begin{array}{l}\text { 8. Je me suis tenu loin de ce qui m'y } \\
\text { faisait penser }\end{array}$ & 0 & 1 & 2 & 3 & 4 \\
\hline $\begin{array}{l}\text { 9. Des images de l'événement } \\
\text { surgissaient dans ma tête }\end{array}$ & 0 & 1 & 2 & 3 & 4 \\
\hline $\begin{array}{l}\text { 10. J'étais nerveux (nerveuse) et je } \\
\text { sursautais facilement }\end{array}$ & 0 & 1 & 2 & 3 & 4 \\
\hline 11. J'essayais de ne pas y penser & 0 & 1 & 2 & 3 & 4 \\
\hline $\begin{array}{l}\text { 12. J'étais conscient(e) d'avoir encore } \\
\text { beaucoup d'émotions à propos de } \\
\text { l'événement, mais je n'y ai pas fait face }\end{array}$ & 0 & 1 & 2 & 3 & 4 \\
\hline $\begin{array}{l}\text { 13. Mes sentiments à propos de } \\
\text { l'événement étaient comme figés }\end{array}$ & 0 & 1 & 2 & 3 & 4 \\
\hline $\begin{array}{l}\text { 14. Je me sentais et je réagissais comme } \\
\text { si j'étais encore dans l'événement }\end{array}$ & 0 & 1 & 2 & 3 & 4 \\
\hline 15. J'avais du mal à m'endormir & 0 & 1 & 2 & 3 & 4 \\
\hline $\begin{array}{l}\text { 16. J'ai ressenti des vagues de sentiments } \\
\text { intenses à propos de l'événement }\end{array}$ & 0 & 1 & 2 & 3 & 4 \\
\hline $\begin{array}{l}\text { 17. J'ai essayé de l'effacer de ma } \\
\text { mémoire }\end{array}$ & 0 & 1 & 2 & 3 & 4 \\
\hline 18. J'avais du mal à me concentrer & 0 & 1 & 2 & 3 & 4 \\
\hline $\begin{array}{l}\text { 19. Ce qui me rappelait l'événement me } \\
\text { causait des réactions physiques telles que } \\
\text { des sueurs, des difficultés à respirer, des } \\
\text { nausées ou des palpitations }\end{array}$ & 0 & 1 & 2 & 3 & 4 \\
\hline 20. J'ai rêvé à l'événement & 0 & 1 & 2 & 3 & 4 \\
\hline 21. J'étais aux aguets et sur mes gardes & 0 & 1 & 2 & 3 & 4 \\
\hline 22. J'ai essayé de ne pas en parler & 0 & 1 & 2 & 3 & 4 \\
\hline
\end{tabular}

Score total : 
Résumé : Troubles post-traumatiques chez des sujets ayant été victimes de violences sexuelles avant l'âge de 15 ans : aspects cliniques, thérapeutiques et médicoéconomiques.

Avant leur majorité, jusqu'à $10 \%$ des femmes et $5 \%$ des hommes sont victimes de violences sexuelles. Le cadre juridique fourni les définitions consensuelles de ces actes. Les données épidémiologiques et de la littérature aident à caractériser les facteurs de risques, la psychopathologie et les comorbidités observés chez les victimes. Ainsi, majoritairement féminines, elles connaissent leur agresseur dans trois quart des cas ; eux, surtout masculin, ont pour la moitié moins de 20 ans. Les troubles rapportés, dont l'état de stress post traumatique, la dépression, les troubles de personnalité et les manifestations somatiques, doivent bénéficier de prises en charge spécifiques suivant plusieurs axes : psychothérapeutique (en première ligne les thérapies cognitivo-comportementales), médicamenteux, mais aussi social et juridique. Des études internationales se sont penchées sur les coûts au sens large de ces agressions. A notre échelle, nous cherchons à évaluer les dépenses directes de santé, chez des sujets ayant été victimes de violences sexuelles avant l'âge de 15 ans. Chez 15 sujets suivis en 2012 à l'hôpital Tenon de Paris, la consommation médicale totale annuelle calculée est dix fois supérieure à la moyenne nationale française, de manière significative et ce malgré notre petit échantillon. Les violences sexuelles sur mineurs, de part leur coût sociétal, financier, moral et psychique, devraient être une préoccupation de santé publique. En ces temps de réflexion budgétaire, des moyens pourraient être donnés aux formations médicales, juridiques et sociales, améliorant le travail de prévention, de repérage et de prise en charge des victimes mais aussi de leur entourage.

Mots clés : Violences sexuelles, troubles post-traumatiques, consommation médicale, impact sociétal.

\section{Abstract: Post-traumatic disorders in patients who have experienced sexual violence before the age of 15: clinical, therapeutic and medico-economic aspects.}

Before their majority, up to $10 \%$ of women and $5 \%$ of men are victims of sexual violence. The legal framework provided consensual definition of this acts. Epidemiological data and publications help us characterize risk factors, the psychopathology and comorbidities observed in victims. Like so, most are women, know their assaulter in three quarter of cases; them, mainly men, have for half, less than 20 years. Disorders reported, including posttraumatic stress disorder, depression, personality disorder and somatic troubles, must have taken specific care, following several axes: psychotherapy (in first line cognitive-behavioral therapy), medication, but also social and juridical. International studies have examined the broad cost of these assaults. At our level, we seek to assess the direct cost for health, in people who have suffered sexual violences before 15 years old. With 15 subjects followed in 2012 at Tenon Hospital in Paris, despite our small sample, the calculated annual total medical consumption is significantly ten times higher then the french national average. By their societal, financial, moral and mental cost, sexual abuse of minors, should be a public health concern. In these times of financial reflexion, funds could be deployed to medical, legal and social training, in order to improve the prevention, screening and care of victims but also their kinfolk.

Key words: Sexual violences, post-traumatic trouble, medical cost, societal impact.

UFR : Université Paris Descartes (Paris V), UFR de Médecine, 15 rue de l'Ecole de Médecine, 75006 Paris. 